\title{
Analysis of the stevor multigene family of Plasmodium falciparum
}

A thesis submitted to the University of London in part fulfillment of the requirements for the degree of Doctor of Philosophy

2002

MALLIKA NIHAL KAVIRATNE

Division of Parasitology

National Institute for Medical Research

(University College, London) 
ProQuest Number: U643591

All rights reserved

INFORMATION TO ALL USERS

The quality of this reproduction is dependent upon the quality of the copy submitted.

In the unlikely event that the author did not send a complete manuscript and there are missing pages, these will be noted. Also, if material had to be removed, a note will indicate the deletion.

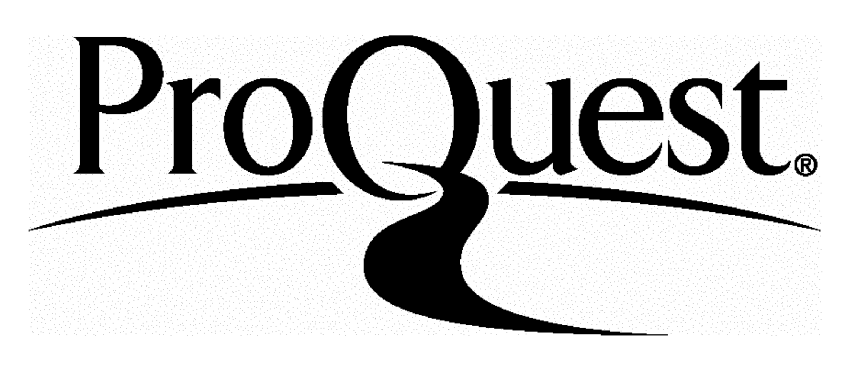

ProQuest U643591

Published by ProQuest LLC(2016). Copyright of the Dissertation is held by the Author.

All rights reserved.

This work is protected against unauthorized copying under Title 17, United States Code. Microform Edition (c) ProQuest LLC.

\author{
ProQuest LLC \\ 789 East Eisenhower Parkway \\ P.O. Box 1346 \\ Ann Arbor, MI 48106-1346
}


For Mummy and Papa 


\section{TABLE OF CONTENTS}

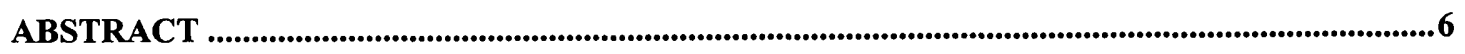

LIST OF FIGURES...........................................................................................................................................

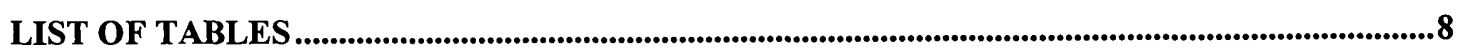

ACKNOWLEDGEMENTS ........................................................................................................................9

ABBREVIATIONS...........................................................................................................................................10

1 INTRODUCTION .........................................................................................................................12

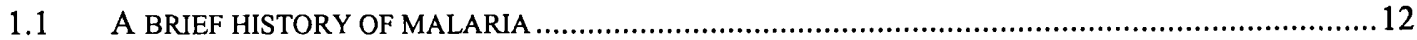

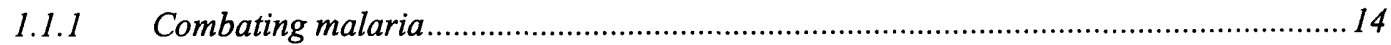

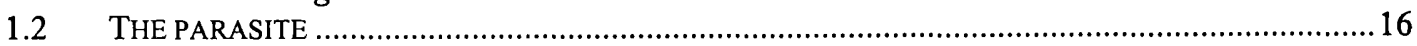

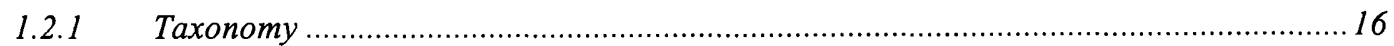

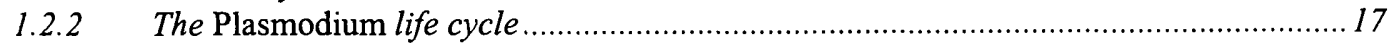

1.3 CLINICAL MANIFESTATIONS AND THE IMMUNE RESPONSE TO MALARIA ...............................19

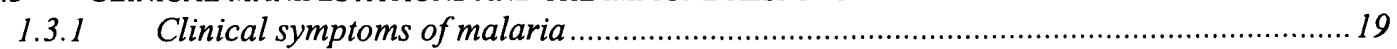

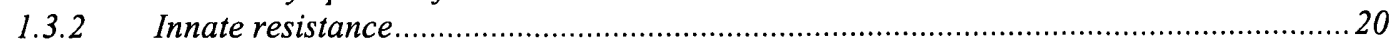

1.3.3 Immune responses directed against the liver stages of infection ...............................21

1.3.4 Immune responses against blood stage malaria infection .......................................21

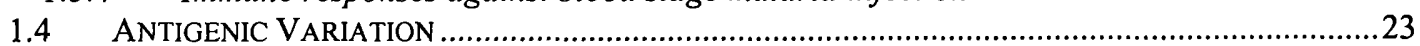

1.4.1 Antigenic variation in malaria..................................................................... 24

1.4.2 Antigenic variation and the immune response in malaria .....................................25

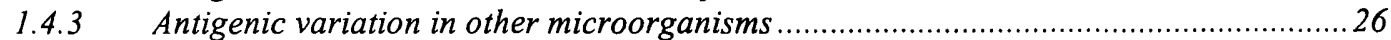

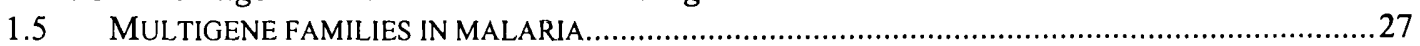

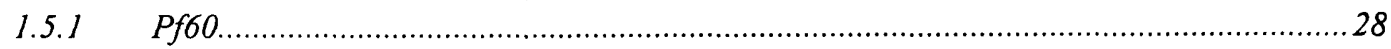

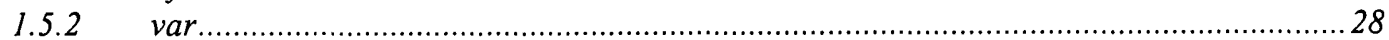

1.5.3 rif

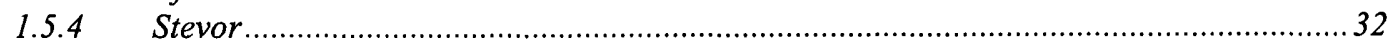

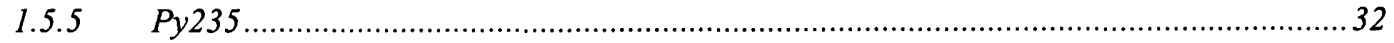

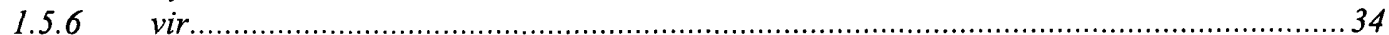

1.5.7 Genomic location of multigene families leads to an increase in the repertoire of genes 34

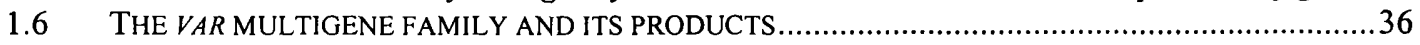

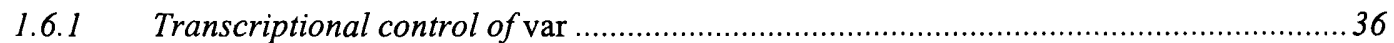

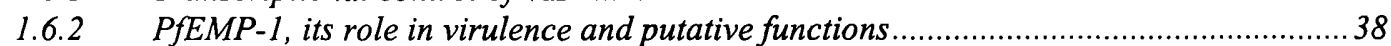

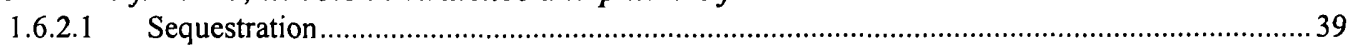

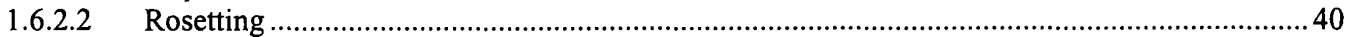

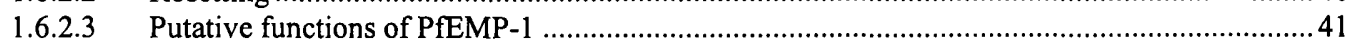

1.6.3 Other proteins associated with the $p R B C$ surface ................................................. 42

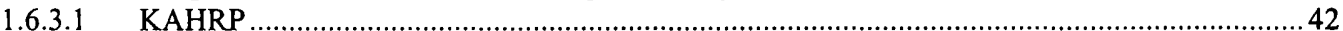

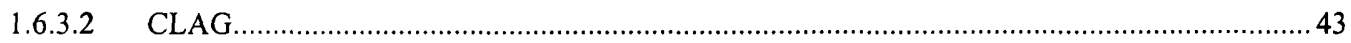

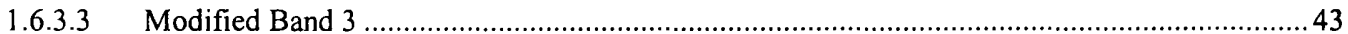

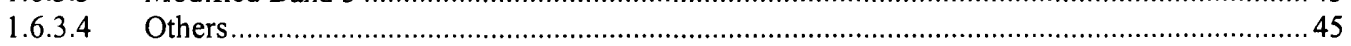

1.7 TRANSPORT OF PROTEINS TO THE ERYTHROCYTE MEMBRANE OF PRBC..............................45

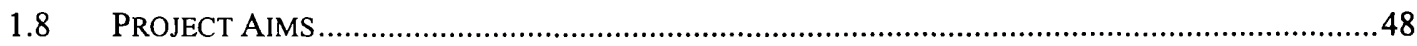

2 MATERIALS AND METHODS ................................................................................................50

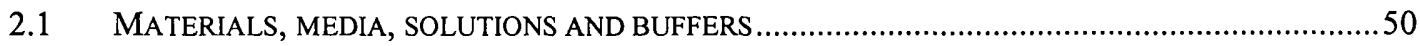

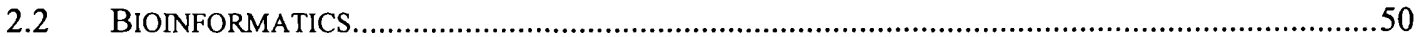

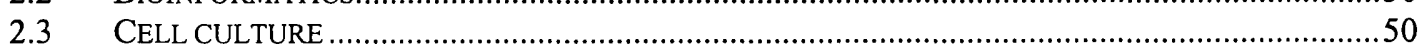

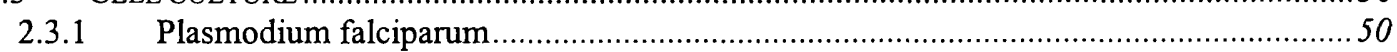

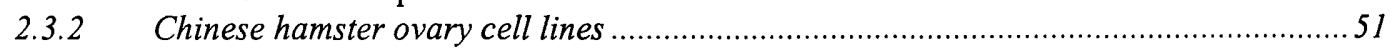

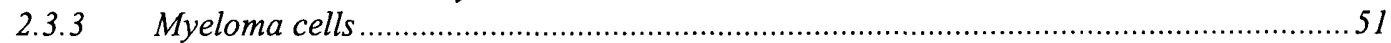

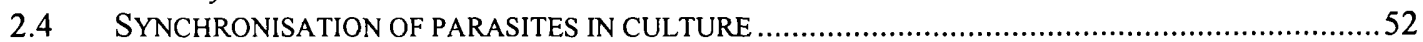

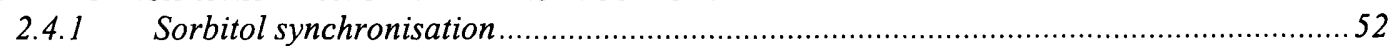

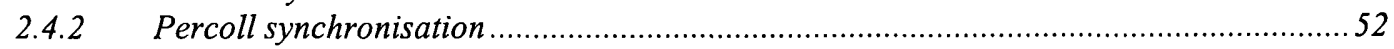




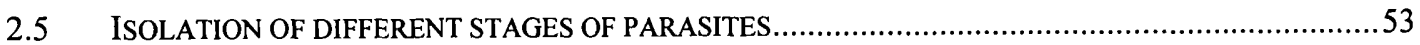

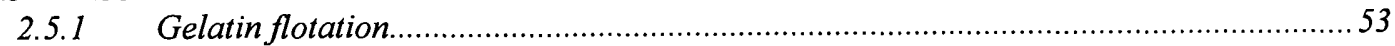

2.5.2 Percoll-sorbitol gradient .................................................................................5

2.6 PREPARATION OF PARASITE GHOSTS FOR IMMUNOFLUORESCENCE ASSAY...............................54

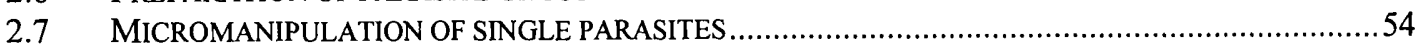

2.8 SELECTION OF CD36-, ICAM-1 - AND CSA-BINDING PARASITES IN VITRO ………...................54

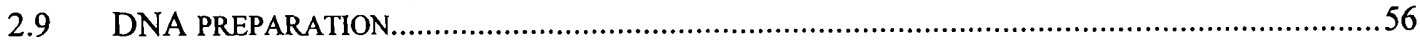

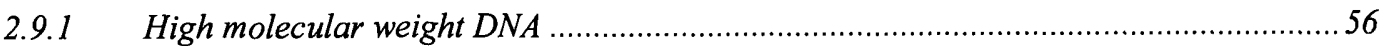

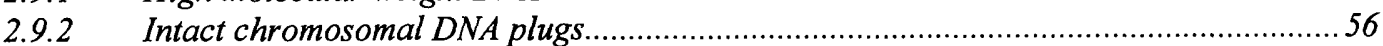

2.10 ISOLATION OF TOTAL RNA FROM PARASITE CULTURE ………….......................................56

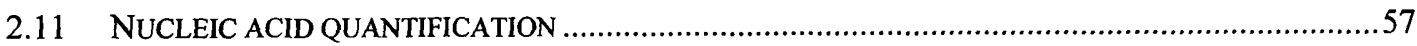

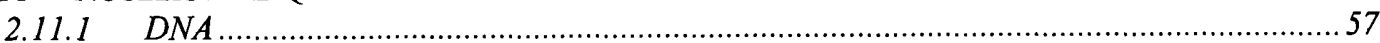

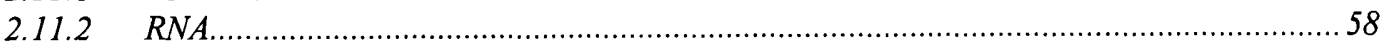

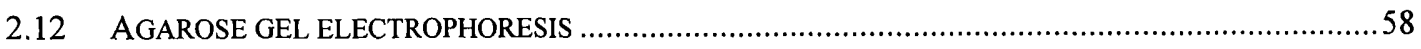

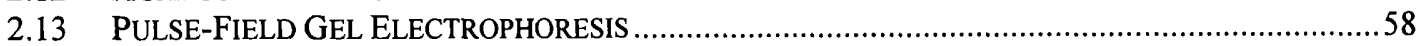

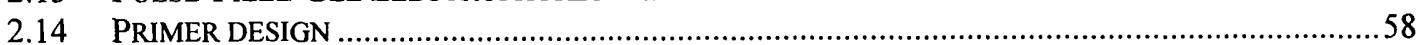

2.15 AMPLIFICATION OF DNA BY POLYMERASE CHAIN REACTION (PCR) …….........................59

2.15.1 PCR using external primers..............................................................................5 59

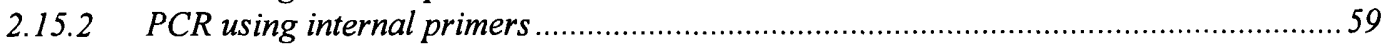

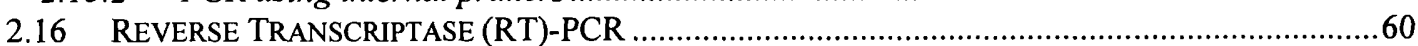

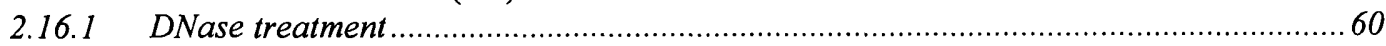

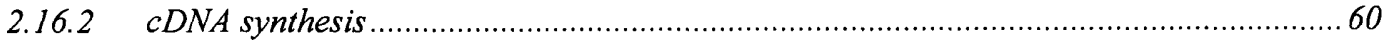

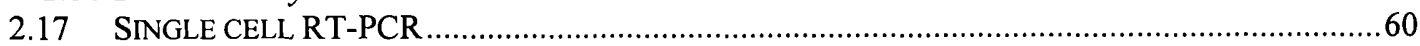

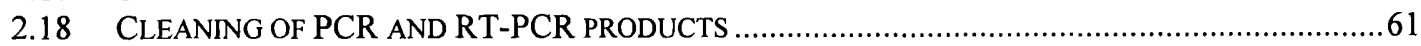

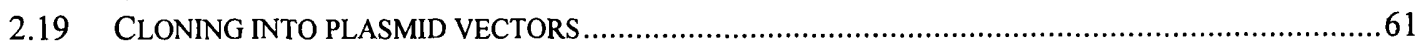

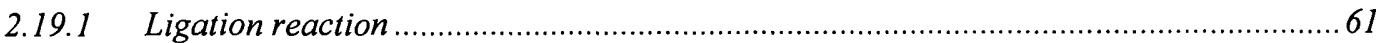

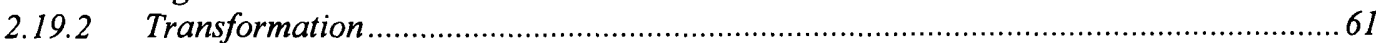

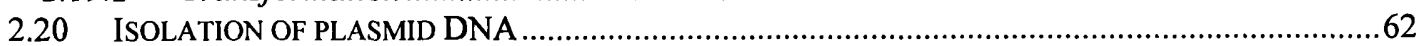

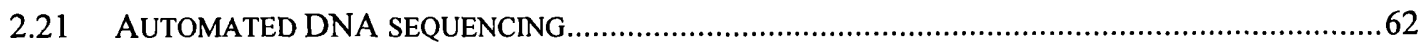

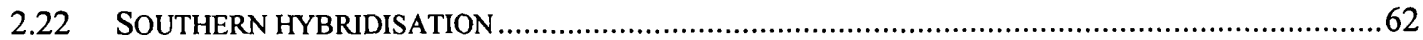

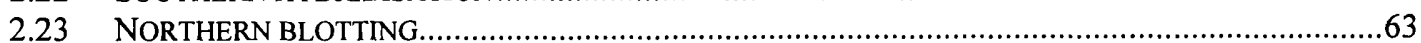

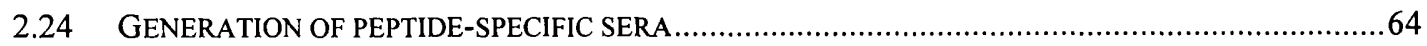

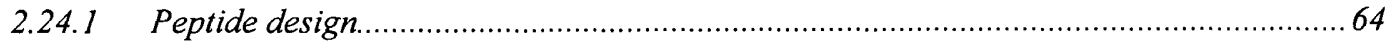

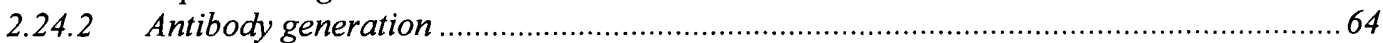

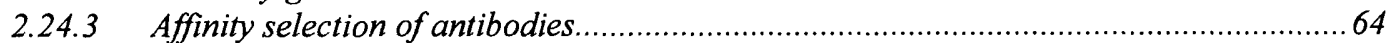

2.24.4 Clearing of serum using RBC lysate ……………...................................................6 65

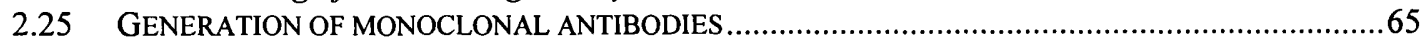

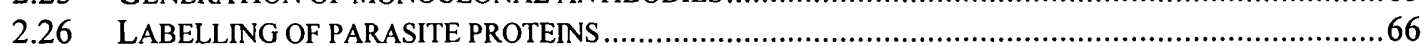

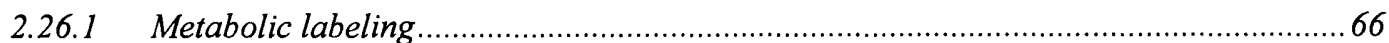

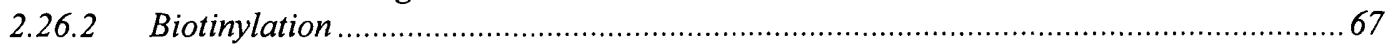

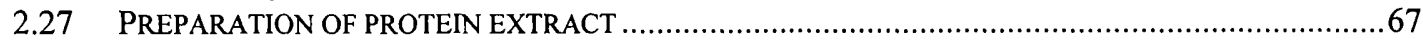

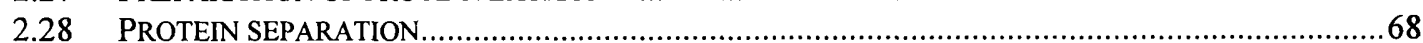

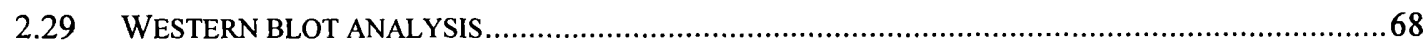

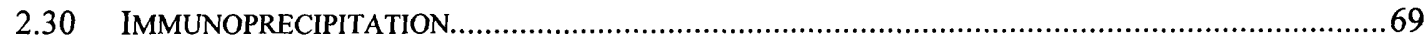

2.31 ENZYME-LINKED IMMUNOSORBANT ASSAY (ELISA) .........................................................70

2.32 INDIRECT IMMUNOFLUORESCENCE ASSAY (IFA) _.................................................................

3 DNA ANALYSIS OF THE STEVOR MULTIGENE FAMILY IN THE 3D7 CLONE OF

P. FALCIPARUM …...............................................................................................................................73

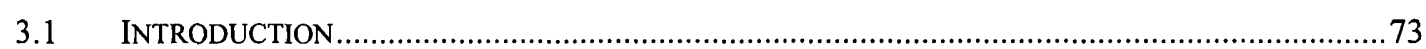

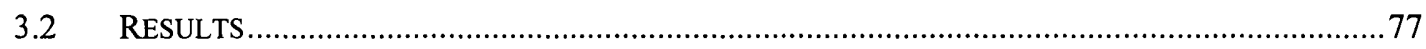

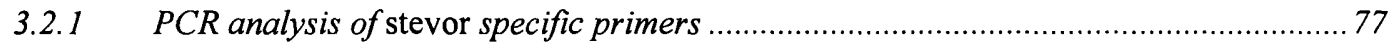

3.2.2 Southern blot analysis of 3D7 chromosomes using stevor specific probes....................77

3.2.3 Repertoire of stevor obtained when using these primer sets........................................ 79

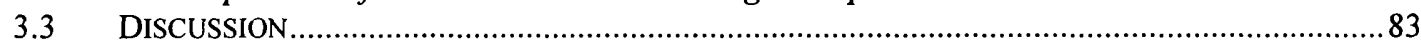

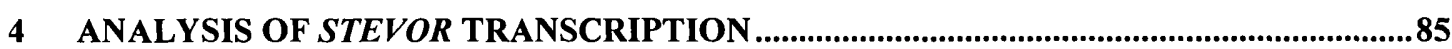

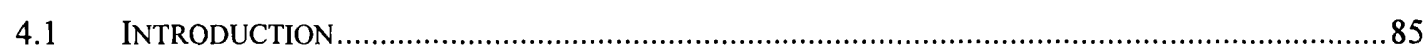

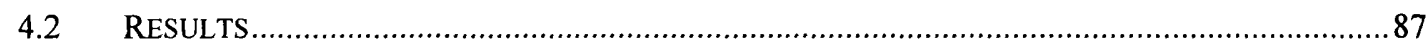

4.2.1 Not all stevor genes are transcribed during asexual blood stages ...............................8 87 
4.2.2 Repertoire of stevor transcripts in $3 D 7$ parasites maintained in vitro. 87

4.2.3 Transcription of stevor occurs within an 8 hour window during the asexual parasite's

48 hour development cycle

4.2.4 Multiple stevor transcripts are detected in single micromanipulated parasites ............ 94

4.2.5 Transcription of stevor is not directly linked to var gene expression...........................95

4.3 DISCUSSION

\section{SUBCELLULAR CHARACTERISATION AND LOCALISATION OF STEVOR IN} P. FALCIPARUM INFECTED ERYTHROCYTES .104

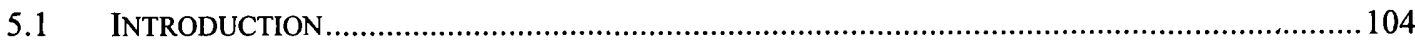

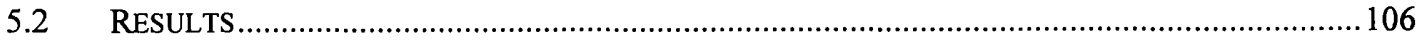

5.2.1 Design of STEVOR peptides in order to generate antibodies ................................ 106

5.2.2 Characterisation of anti-STEVOR antibodies using ELISA.................................... 108

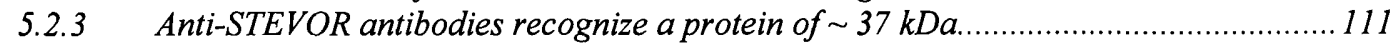

5.2.4 Antibodies against STEVOR react with fixed 3D7 trophozoites and schizonts ........... 119

5.2.5 STEVOR is located in the Maurer's Clefts ............................................................ 124

5.2.6 STEVOR is also expressed in gametocytes ......................................................... 131

5.2.7 Is STEVOR on the surface of $p R B C$ ? ................................................................. 131

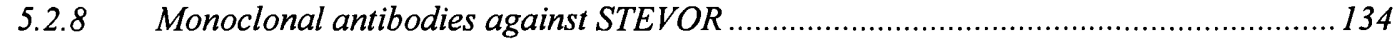

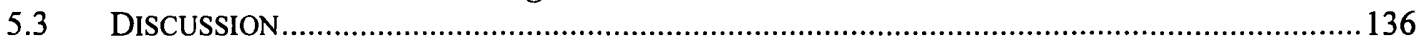

6 FINAL SUMMARY AND FUTURE WORK .................................................................. 141

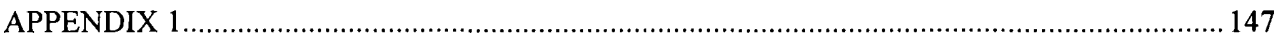

APPENDIX 2 2

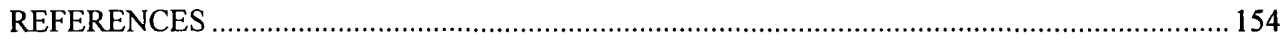




\begin{abstract}
In their natural hosts malaria parasites cause long lasting chronic infections. This is in spite of a vigorous immune response by the host. The ability of the parasite to antigenically vary is thought to be one of the main reasons for this observation. Several multigene families have been described in the human parasite Plasmodium falciparum and they have been linked to antigenic variation and the pathology associated with malaria. The stevor multigene family of $P$. falciparum has only been partially characterised. Around 34 copies of the stevor gene family are found on all 14 chromosomes of the parasite genome. The overall structure and size of all stevor genes is conserved. Each gene codes for an approximately $30-40 \mathrm{kDa}$ protein, consists of two exons and has 2-3 predicted transmembrane regions.

In an attempt to get a better understanding of how the parasite utilises stevor, I have studied this gene family at the DNA, RNA and protein level. Stevor is transcribed during a very tight window of the parasite life cycle. However, only a subset of the genes is transcribed in cultured parasites and this is reflected in single micromanipulated parasites. Finally, there does not appear to be a direct link between stevor and var transcription. Antibodies raised against different regions of STEVOR recognise proteins of $\sim 37 \mathrm{kDa}$ and locate STEVOR within discrete sites (Maurer's clefts) of the infected red blood cell in late trophozoites and schizonts. Moreover, STEVOR is also expressed in gametocytes. These results suggest that STEVOR many have a novel role in $P$. falciparum biology.
\end{abstract}




\section{LIST OF FIGURES}

Figure 1.1 Geographical map showing the distribution of malaria world-wide

Figure 1.2 Life cycle of Plasmodium spp.

Figure 1.3 A consensus for the arrangement of repetitive DNA sequences on $\mathrm{P}$.

falciparum telomeres

Figure 1.4 A schematic comparing stevor and rif

Figure 1.5 Schematic representation of a $P$. falciparum infected RBC membrane

Figure 1.6 Schematic of putative trafficking pathways in malaria-infected

Figure 2.1 Percoll/sorbitol separation of different stages of $P$. falciparum-infected

$\mathrm{RBC}$

Figure 3.1 Diagram of the organisation of 3D7 stevor genes on the 14 chromosomes of $P$. falciparum

Figure 3.2 Schematic representation of stevor and the position of primers

Figure 3.3 PCR analysis of stevor

Figure 3.4 Alignment of stevor unique sequences

Figure 3.5 Analysis of DNA sequences from cloned stevor PCR products

Figure 4.1 RT-PCR analysis of stevor

Figure 4.2 Comparison of stevor sequences from cloned PCR and RT-PCR 90

products

Figure 4.3 Timing of stevor transcription

Figure 4.4 RT-PCR analysis of time course RNA samples 96

Figure 4.5 RT-PCR of single cell micromanipulated trophozoites 97

Figure 4.6 Analysis of stevor transcription by PCR and Southern blot of 99

unselected parasites or parasites selected on different ligands

Figure 5.1 Production of anti-STEVOR antibodies-peptide design.

Figure 5.2 An ELISA demonstrating the binding of anti-peptide 1 serum 109

Figure 5.3 An ELISA demonstrating the binding of anti-peptide 2 serum 112

Figure 5.4 An ELISA demonstrating the binding of anti-peptide 3 serum 114

Figure 5.5 Binding of immune serum from rabbit 6344 to Western blots and $\quad 116$

affinity purification of the serum.

Figure 5.6 Expression of STEVOR in $P$. falciparum 3D7 parasites. 117

Figure 5.7 Western using anti-peptide 2 and anti-peptide 3 serum 118

Figure 5.8 IFA of fixed $P$. falciparum 3D7-infected RBC of late trophozoites and $\quad 120$

schizonts

Figure 5.9 IFA of fixed $P$. falciparum 3D7-infected red cells using mouse serum against peptide (pep) 2. and pep 3

Figure 5.10 Co-localisation IFA of rabbit anti-peptide 1 and mouse anti-peptide 3

serum

Figure 5.11 Co-localisation IFA with anti-peptide 1 serum and anti-rhoptry

antibodies

Figure 5.12 Co-localisation IFA with anti-peptide 1 serum and anti-PfSPB-1

serum

Figure 5.13 IFA using fixed $P$. falciparum 3D7 schizont ghosts

Figure 5.14 IFA using fixed $P$. falciparum 3D7 PfEMP-3 truncated schizonts $\quad 130$

Figure 5.15 IFA using fixed $P$. falciparum 3D7 gametocytes 132

Figure 5.16 Biotinylation of RBC P. falciparum 3D7 schizont stage pRBC 133

Figure 5.17 Western using $\mathrm{mAb}$ specific for peptide 1 


\section{LIST OF TABLES}

Table 2.1 Lambda HindIII marker

Table 2.2 Stevor-specific primers

Table 2.3A Primary antibodies used in IFA

Table 2.3B Anti-STEVOR peptide antibodies

Table 2.4 Secondary antibodies used in IFA 


\section{ACKNOWLEDGEMENTS}

There are many people whom I would like to thank for helping me during the course of my PhD. Firstly, I would like to thank Dr. Peter Preiser who has been an excellent supervisor and has encouraged and supported me throughout with his help and enthusiasm. I am particularly indebted to Dr. Shahid Khan who despite providing an endless battery of insults has shown me how to do just about everything especially with regards to molecular biology and without whom this $\mathrm{PhD}$ would not have been as enjoyable. Also, thank you for running the Northern and performing the selection of parasites. A special thanks to Dr. Bill Jarra who has helped me throughout but especially in his dealings with small furry animals on my behalf, and for his help with the monoclonal antibodies, sequencing and micromanipulation.

Many people have given me technical and practical help. I would especially like to thank Dr. Mike Blackman for his help with the protein work and involved in this project and useful discussions on how to proceed as well as critical reading of this report. I would like to thank the following people for providing me with parasites and other reagents; Mrs. Muni Grainger (parasites), Dr. Isabelle Delrieu (parasites), Mr. Terry Scott-Finnigan (antibodies), Ms. Sola Ogun (antibodies) and Mr Justin Pachebat (RNA). Dr. Ching Li for helping me with ELISAs, Dr Deirdre Cunningham for help with BioEdit and supporting me through me last minute panics. I would also like to thank Dr Tony Holder for being my second supervisor, for his advice in general matters and for critical reading of this report. A special thanks to Dr. Helen Taylor who has provided me all kinds of help whenever I have needed it.

I would also like to thank Mrs. Fiona Hackett for her friendship and support and for dragging me out running in the freezing cold. Ms. Jane Shepley for keeping me amused and for keeping the female end-up in the lab during my second year and also Ms. Katy Fuller. Thank you to everyone in Parasitolgy for all their help and for making my $\mathrm{PhD}$ such a memorable experience.

Finally I would like to thank Smita for looking after me and providing me with excellent meals when I most needed them, Stuart for his tremendous patience and love during my $\mathrm{PhD}$ and most importantly to my parents for their support, encouragement and understanding.

This work was funded by the Medical Research Council. 


\section{ABBREVIATIONS}

\section{Most used abbreviations}

\begin{tabular}{|c|c|}
\hline aa & Amino acid \\
\hline APC & Antigen Presenting Cell \\
\hline BES & Blood stream expression site \\
\hline BSA & Bovine Serum Albumin \\
\hline CD & Cluster Differentiation \\
\hline cDNA & Complementary DNA \\
\hline CHO & Chinese Hamster Ovary \\
\hline CIDR & Cysteine-rich interdomain Region \\
\hline CLAG & Cytoadherence linked asexual gene \\
\hline $\mathbf{C M}$ & Cerebral malaria \\
\hline CR1 & Complement receptor 1 \\
\hline CSA & Chondroitin sulphate A \\
\hline CSP & Circumsporozoite protein \\
\hline DAPI & 4,6-diamidino-2-phenylidole \\
\hline DBL & Duffy binding like \\
\hline $\mathrm{dd} \mathrm{H}_{2} \mathrm{O}$ & Double-distilled water \\
\hline DNA & Deoxyribose nucleic acid \\
\hline ECL & Enhanced chemiluminesence \\
\hline EDTA & Ethylenediaminetetraacetic acid \\
\hline ELISA & Enzyme-linked Immunoabsorbant Assay \\
\hline ER & Endoplasmic reticulum \\
\hline EtBr & Ethidium bromide \\
\hline FITC & Fluorescein Isothiocyanate \\
\hline GFP & Green fluorescent protein \\
\hline GPA & Glycophorin A \\
\hline GST & Glutathione S-transferase \\
\hline HAT & Hypoxanthine Aminopterin Tetracycline \\
\hline HLA & Human Leukocyte antigen \\
\hline HRP & Horse radish peroxidase \\
\hline ICAM-1 & Intercellular adhesion molecule-1 \\
\hline IFA & Indirect immunofluorescence assay \\
\hline Ig & Immunoglobulin \\
\hline IL & Interleukin \\
\hline KAHRP & Knob associated histidine rich protein \\
\hline KBS & Krebs Buffered Saline \\
\hline kDa & Kilodalton \\
\hline KLH & Keyhole limpet haemocyanin \\
\hline $\mathbf{m A b}$ & Monoclonal antibody \\
\hline $\mathbf{M B q}$ & Mega-bequerels \\
\hline $\mathbf{M C}$ & Maurer's cleft \\
\hline MHC & Major histocompatibilty complex \\
\hline MSP-1 & Merozoite surface protein-1 \\
\hline $\mathrm{NaOH}$ & Sodium hydroxide \\
\hline OD & Optical density \\
\hline PAGE & Polyacrylamide gel electrophoresis \\
\hline PBS & Phosphate Buffered Saline \\
\hline PCR & Polymerase Chain Reaction \\
\hline PfEMP-1 & Plasmodium falciparum extracellular membrane protein- 1 \\
\hline PFGE & Pulse Field Gel Electrophoresis \\
\hline PfSBP-1 & Plasmodium falciparum skeletal binding protein 1 \\
\hline $\mathbf{P M}$ & Plasma membrane \\
\hline PNPP & p-Nitrophenylphosphate-sodium \\
\hline PVM & Parasitophorous vacuolar membrane \\
\hline (p)RBC & (Parasitised) red blood cell \\
\hline RESA & Ring-associated erythrocyte surface antigen \\
\hline
\end{tabular}


RIF

RNA

RSP

RT

RT-PCR

SDS

SICA

STEVOR

Taq

TBE

TE

TNF

TR

TRITC

TSP

TVN

U

UTR

$\mathbf{v} / \mathbf{v}$

VCAM

VESA

VMP

VSG

$\mathbf{w} / \mathbf{v}$

WHO

xg

YAC
Repetitive interspersed family

Ribose nucleic acid

Ring surface protein

Reverse transcriptase

Reverse Transcriptase Polymerase Chain Reaction

Sodium Dodecyl Sulphate

Schizont infected cell agglutination

Subtelomeric variable open reading frame

Thermus aquaticus

Tris Borate EDTA

Tris EDTA

Tumour necrosis factor

Texas Red

Tetramethyl-rhodamine isothiocyanate

Thrombospondin surface protein

Tubo-vesicular network

Unit

Untranslated region

Volume per volume

Vascular cell adhesion molecule

Variant erythrocyte surface antigen

Variable major protein

Variant surface glycoprotein

Weight per volume

World Health Organisation

Gravity

Yeast Artificial chromosome 


\section{CHAPTER ONE}

\section{Introduction}

\subsection{A brief history of malaria}

Malaria is one of the most prominent diseases affecting human populations in tropical and subtropical areas of the world. Historically, the distribution of malaria was more widespread globally and it is only due to climatic changes and human intervention that it is now limited to certain regions of the world (Figure 1.1). Heroditus of ancient Rome was amongst the first recorded people to directly link the expansion of cities into marshy areas with an increase in malaria epidemics. Unfortunately, two thousand years later, the threat of malaria is still greater than ever (Garrett, 1994).

The disease is caused by parasitic protozoa of the genus Plasmodium that are transmitted to humans by the female Anopheles mosquito. The World Health Organisation (WHO) estimate that 2.5 billion people in approximately 90 different countries worldwide are currently exposed to the disease (Figure 1.1). Up to 500 million cases of malaria occur annually resulting in the death of 1-2 million people, the majority of whom are children under the age of five.

The first major advance in man-kind's attempts to combat malaria were with the pioneering work of Charles Laveran who identified the Plasmodium parasite as the causative organism of malaria. The second was the recognition, widespread use and development of the chemotherapeutics of Quinine and its synthetic derivatives. In the 1960's and 70's the threat of malaria was temporarily abated in many parts of the world. This was due to the concerted efforts by the WHO to eradicate the mosquito vector by spraying with the insecticide DDT (dichloro-diphenyl-trichloroethane) as well as combating the disease with chloroquine. This effort had the most visible effect on controlling malaria to date (Jayaraman, 1997). However, although initially insecticide control was cheap and effective, insecticide resistance in Anopheles and increased ecological awareness has limited its use and effectiveness. More recently, the introduction of insecticide impregnated bed nets has also been reported in some cases to reduce infection rates and deaths by one third (D'Alessandro et al., 1995). 


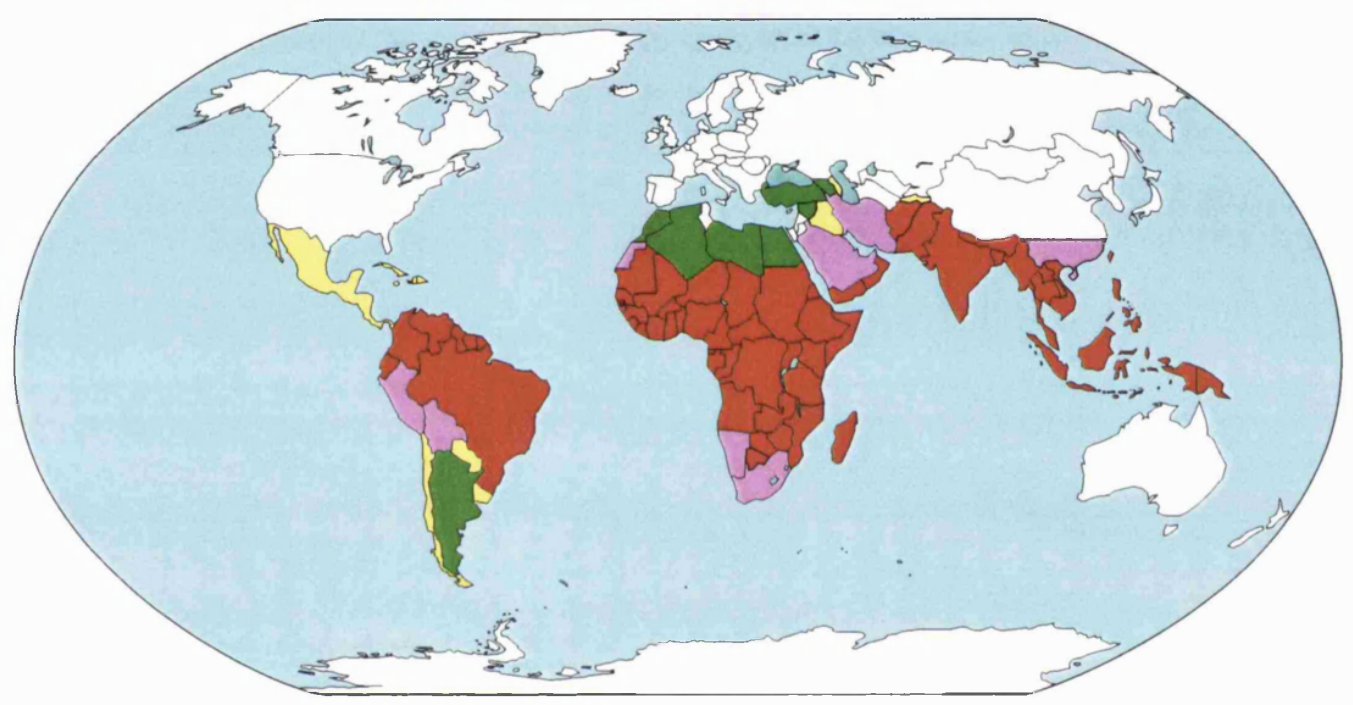

Figure 1.1 Geographical map showing the distribution of malaria world-wide. ( ) countries where malaria is present only in small remote areas. (1) intermediate malaria risk, no chloroquine resistant strains. ( $)$ intermediate malaria risk, but choloroquine resistance is confirmed for $P$. falciparum. $(\square)$ high risk area for chloroquine resistance $P$. falciparum. Modified from www.world66.com/TravelWise. 
Unfortunately, all of these initial successes have thrown up or have been followed by major setbacks. Alarmingly, malaria is thus once again on the increase due to the emergence of mosquitoes resistant to insecticides and parasites resistant to antimalarial drugs. Additionally, the change in the world climate also threatens to reintroduce malaria into areas, including continental Europe, where it was previously eradicated.

The WHO has placed malaria at the top of its agenda. The 1998 "Roll Back Malaria" initiative aims to upgrade and redistribute health facilities, intensify the use of bednetting and facilitate research into new drugs (WHO, 1999). In addition, another programme, the Malaria Vaccine Initiative was created with the objective of significantly accelerating the clinical development of promising malaria vaccine candidates. The production and widespread application of an efficient malaria vaccine would represent a major advance in the fight against the disease.

\subsubsection{Combating malaria}

Anti-malarial prophylactic drugs currently form the basis for control of the disease in individuals. However, due to inappropriate or careless use of anti-malarial drugs, high levels of resistance to these drugs prevail in many countries and multi-drug resistant strains of the parasite have now emerged (Trigg and Kondrachine, 1998). This has led to the urgent need for the development of affordable new drugs or potentially immunisation with a vaccine against the parasite.

Although drugs now form the basis of control of the disease in individuals and can be used as prophylactics especially for travellers to areas where malaria is prevalent, it is not economically viable for those living in endemic regions to take such drugs continuously. Drug resistance will also continue to be a major pitfall in this approach and therefore the current drive is toward development of a malaria vaccine.

The observation, first made in 1973, that immunisation with irradiated sporozoites protected naïve volunteers against infection provided strong support for the belief that an effective malaria vaccine will be achievable (Clyde et al., 1973). Since then, a great deal of research has been directed towards the development of a malaria vaccine (reviewed in Anders and Saul, (2000) and Holder, (1999)). However, malaria vaccine research has been hampered by the complexity of the life cycle, imperfect tools 
to assess the efficacy of immune responses and limited knowledge and understanding of the factors that determine the outcome of an infection.

Three main types of vaccines are being developed: anti-sporozoite vaccines, anti-asexual blood stage vaccines and transmission blocking vaccines. Initial research has concentrated on anti-sporozoite vaccines designed to prevent infection. Early trials were based on subunit vaccines against circumporozoite protein (CSP), the major sporozoite surface protein but despite several trials carried out over the following 10 years, there was limited success due to poor antibody responses in humans (Herrington et al., 1987; Ballou et al., 1987). More recently, the RTS,S vaccine consisting of a recombinant hepatitis B surface antigen vaccine incorporating CSP sequences, tested in a field trial, was found to reduce prevalence of parasitaemia in adults by $65 \%$ (Bojang et al., 2001). However, the vaccine was only effective for 2 months and research is now focused on improving efficacy of this vaccine.

The asexual blood stage is responsible for the pathology of the disease, and vaccines developed against this stage are designed to reduce severe and complicated manifestations of the disease. There are several asexual-blood stage vaccine candidates including apical membrane antigen-1, erythrocyte binding antigen 175 and rhoptry associated protein 1 . One of the leading blood stage candidates is merozoite surface protein (MSP)-1 and certain anti-MSP-1 antibodies have been found to interfere with the asexual cycle by preventing invasion of merozoites into erythrocytes (Holder et al., 1994). Recent vaccine trials in monkeys have shown that protection against a lethal challenge infection of $P$. falciparum was elicited in malaria-naive Aotus monkeys by vaccination with the $\mathrm{C}$ terminus 19-kDa protein of MSP-1 (Egan et al., 2000). Once inside the red blood cell ( $\mathrm{RBC}$ ), parasite-derived proteins can be expressed on the surface of the parasitised $\mathrm{RBC}$ ( $\mathrm{pRBC}$ ). These surface proteins are implicated in the binding of $\mathrm{pRBC}$ to the vessels of the microvasculature of the brain, a phenomenon that is associated with the onset of cerebral complications (see Section 1.6.2). These proteins, also being examined as potential vaccine candidates, undergo clonal antigenic variation making the task of developing an effective vaccine very difficult (Gamain $e t$ al., 2001).

Transmission blocking vaccines interrupt the development of parasite gametes in the mosquito. Although these vaccines do not protect the individual against infection and/or disease, they are designed to reduce transmission of the disease. Antibodies to 
gamete proteins have been found to block transmission by mosquitoes to mice and rabbits, and human trials are anticipated to begin shortly (Taubes, 2000).

A vaccine targeting liver stage antigens, blood stage antigens and a transmission-blocking component would be ideal. Shi et al., (1999) have produced a recombinant protein by expression of a synthetic gene that codes for short peptide sequences derived from two sporozoite antigens, one liver stage antigen, five asexual blood stage antigens and one gametocyte protein. Although antibodies raised against this recombinant protein react with sporozoites, liver and blood asexual stages and gametocytes in in vitro assays, it is unclear how these in vitro assays relate to in vivo functionality.

DNA vaccines may also combine a variety of antigens from different stages of the life cycle to elicit maximal immune responses. DNA vaccines encoding up to 15 different malaria proteins have been used to induced both antibody and cytotoxic $\mathrm{T}$ cell responses in animal studies, attacking the malaria parasite in the blood stream as well as in the hepatocyte (Taubes, 2000). Phase I safety and immunogenicity trials of a multiepitope DNA vaccine have recently been completed (Ferry, 2000). The current status of multi-stage DNA vaccine is reviewed in (Doolan and Hoffman, 2001). However, as of yet no effective long-term vaccine against malaria has been developed.

\subsection{The parasite}

\subsubsection{Taxonomy}

Plasmodium spp. belong to the phylum Apicomplexa, a group of parasitic protozoa characterised by a complex group of organelles (the apical complex), present in the invasive stages of these parasites. Other members of this phylum include the AIDS-related opportunistic human pathogens Toxoplasma and Cryptosporidium, as well as the causes of important veterinary diseases including Theileria spp., Babesia spp. and Eimeria spp.

There are over one hundred species of Plasmodium found in the blood of reptiles, birds and mammals (Garnham, 1973). There are four species of Plasmodium that naturally infect humans: P. falciparum, P. vivax, P. ovale, and P. malariae, of which $P$. falciparum is the most prevalent and is associated with the greatest pathology 
and most if not all of the mortality. Each of the species differs in morphology, epidemiology and clinical presentation, but they all have similar life cycles.

Both the human and non-human Plasmodium spp. are important experimental models, including $P$. falciparum in Aotus monkeys and $P$. vivax in chimpanzees as well as the simian malarias such as $P$. cynomolgi and $P$. knowlesi and the rodent malarias P. berghei, P. chabaudi and P. yoelii.

\subsubsection{The Plasmodium life cycle}

Plasmodium has a complicated life cycle (Figure 1.2), residing in two hosts. Asexual development and replicative cycles take place in the vertebrate host, whereas sexual development occurs in the mosquito vector.

A malaria infection is initiated when an infected mosquito takes a blood meal. Injected sporozoites are present in the blood stream for only a short time as they invade liver cells within minutes of introduction into the human host. The sporozoite develops into a hepatic trophozoite, which then multiplies to form multi-nucleated hepatic schizonts within which develop thousands of merozoites. Thus, theoretically only a single sporozoite is required to trigger a potentially fulminating infection. In the case of $P$. vivax and $P$. ovale, some sporozoites become hypnozoites, which can lie dormant in hepatocytes causing the disease to relapse months or years later (Gilles and Warrel, 1993).

Upon rupture of the hepatic schizont, thousands of mature merozoites are released back into the blood where they invade $\mathrm{RBC}$ within seconds. At this stage the parasite consists of a well-defined nucleus and scant cytoplasm resembling a "ring" (in Giemsa stained preparations) and is located within a parasitophorous vacuole formed during the invasion process. The "ring" stage parasites mature into trophozoites, which then undergo asexual division termed schizogony to yield between 8 and 32 merozoites (depending on the species of malaria) that are released by the rupture of the RBC membrane. Most merozoites rapidly invade uninfected RBC to initiate the next round of intra-erythrocytic replication. In $P$. falciparum the erythrocytic asexual cycle takes around $48 \mathrm{~h}$ and schizogony is synchronous within the host. The pathology of malaria is only associated with this stage of the life cycle.

A small proportion of merozoites, following $\mathrm{RBC}$ invasion, differentiate into male or female gametocytes in a process called gametogenesis which after maturation 


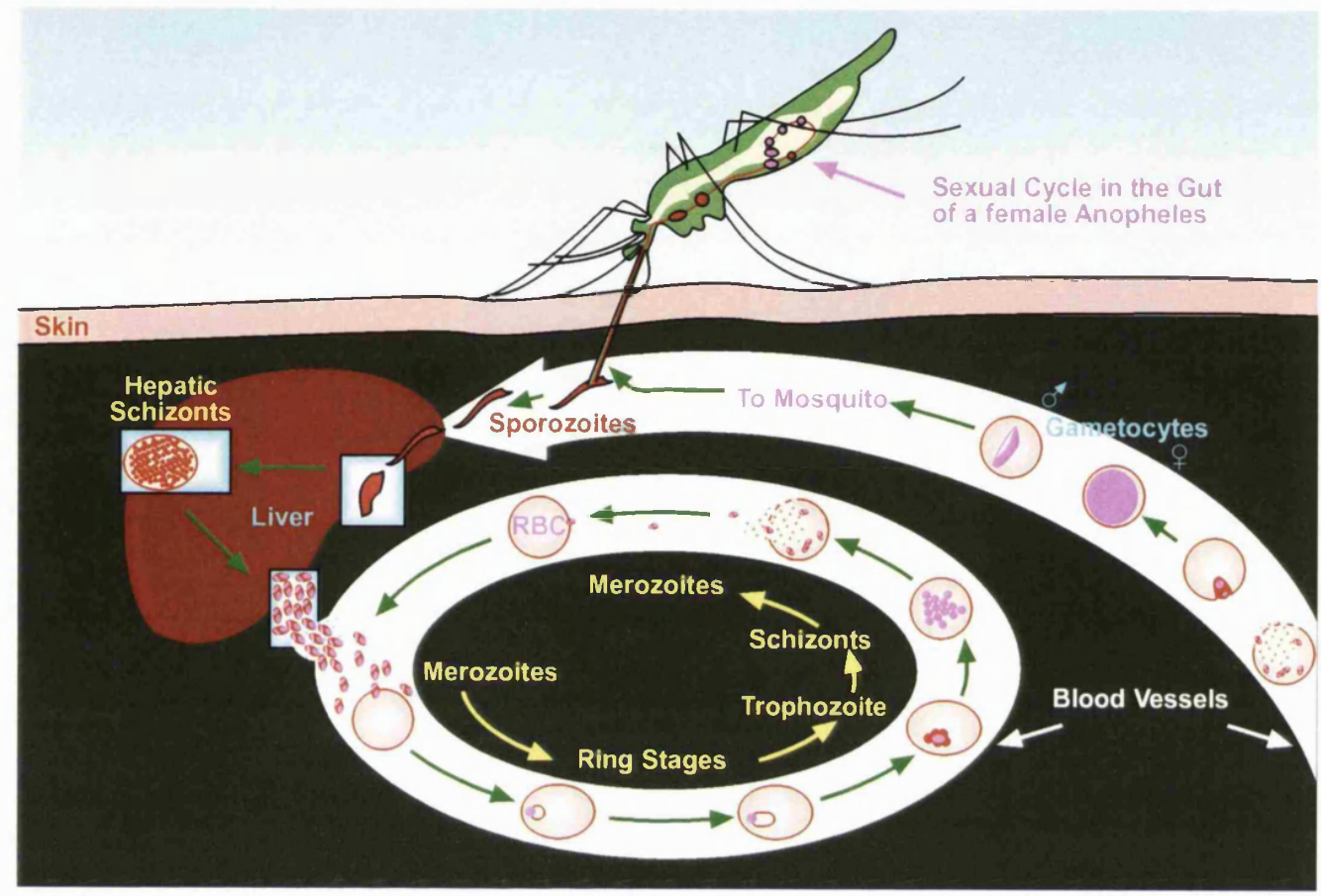

Figure 1.2 Life Cycle of Plasmodium spp. There are two distinct phases: sexual (in the mosquito) and asexual (in the mammalian host). An infected mosquito feeds and injects sprorozoites into the bloodstream. Sporozoites invade hepatocytes and undergo growth and asexual division to form hepatic shizonts. Infected hepatocytes burst and release merozoites into the blood stream. Merozoites invade red blood cells (RBC), develop through ring and trophozoite stages and undergo schizogony to form multinucleate schizonts which then burst, releasing merozoites that invade new RBC. After invasion, some merozoites undergo sexual differentiation to form gametocytes and these are then taken up by feeding mosquitoes to produce female and male gametes in the mosquito gut which fuse to form zygotes. The motile zygote (ookinete) penetrates the stomach wall and an oocyst develops. Inside the oocyst repeated divisions (sporogony) produce many sporozoites. These migrate to and mature in the mosquito's salivary glands from where they are transmitted during feeding. Modified from www.malaria.org/lifecycle.html. 
can circulate in the peripheral blood for extended periods. These are then taken up by the mosquito during a blood meal, where male and female gametoytes differentiate into haploid gametes and fuse to form a diploid zygote in the mosquito gut. Zygotes can result from selfing events (i.e. fertilisation with gametes derived from the same parent) or from cross fertilisation between different parasite genotypes if there is a multiple infection (more than one strain of parasite) in the host. The zygote undergoes a meiotic division to become a motile ookinete, which embeds itself into the mosquito midgut wall and develops into an oocyst. In the developing oocyst, mitotic division gives rise to large numbers of sporozoites that eventually burst out and migrate to the salivary glands maturing while in transit. Mature sporozoites in the salivary glands are then injected into the mammalian host when the mosquito takes its next blood meal completing the life cycle (Gilles and Warrel, 1993).

Exponential growth of parasites in RBC, the modifications of $\mathrm{pRBC}$ in terms of parasite proteins expressed on the RBC surface and the concomitant immune response to the parasite, ultimately result in the disease manifestations of clinical malaria.

\subsection{Clinical manifestations and the immune response to malaria}

\subsubsection{Clinical symptoms of malaria}

Symptoms of malaria are caused by two factors, one of which is parasite derived and the other is immune-mediated. Fever, with accompanying nausea, headaches and chills are the most prevalent symptoms of malaria. Clinical complications occur particularly in non-immune adults and young children who remain untreated. Severe forms of disease encompass a range of presentations including severe anaemia, liver failure, hypoglycaemia, acute pulmonary oedema, renal failure and cerebral malaria. Substantial mortality in P. falciparum infection is attributed to cerebral malaria (CM) which kills up to $30 \%$ of clinical cases even during treatment. Obstruction of cerebral venules and capillaries by $\mathrm{pRBC}$ is a characteristic finding in postmortem studies of patients with cerebral malaria (MacPherson et al., 1985; Pongponratn et al., 1991).

However, despite the pathology induced by $P$. falciparum, individuals living in endemic countries, as a result of repeated infections, no longer show any clinical or 
pathological symptoms of malaria suggesting that some level of protective immunity is eventually acquired.

\subsubsection{Innate resistance}

In human populations, which are genotypically and phenotypically very diverse, innate resistance may play a role in host specificity Plasmodium parasites. The innate factors that operate at the level of the erythrocytic cycle include the presence or absence of appropriate receptors for the parasite (Miller et al., 1975), the age of the red blood cell (Craik, 1920), and haemoglobin type (Allison, 1954). Thus, individuals whose erythrocytes lack the Duffy blood group appear to be completely resistant to infection with $P$. vivax (Miller et al., 1976; Spencer et al., 1978). Malaria may influence certain phenotypes attributable to an alteration in gene frequencies in human populations. It has been suggested that the high frequencies of thalassaemia and other haemoglobinopathies observed in malaria endemic areas resulted from the fact that these genetic disorders offered some protection against the parasite (Nagel, 1990). The sickle cell trait also exists in a state of balanced polymorphism in malaria endemic areas where the disadvantage of having a defective haemoglobin gene allowed an improved survival rate for the carrier (Lell et al., 1999). Innate resistance is not always absolute and genetically determined factors can play a role in reducing the severity of infection and improve survival. In some rodent and human malarias the age of the RBC is a particularly critical factor: although certain parasites invade both mature and immature red blood cells (e.g. P. falciparum and P. chabaudi) (Jarra and Brown, 1989) whereas others preferentially invade reticulocytes (e.g. P. vivax) (Kitchen, 1938).

More recently, expression of different human major histocompatability (MHC) complexes; class I and II have been correlated with protection to $P$. falciparum infection (Hill et al., 1991). This study suggested that HLA class I antigen, HLA-B53, and certain HLA class II haplotypes of DRB1, were associated with reduced susceptibility to severe malaria. Interestingly, both these haplotypes are much more frequent in West Africans than other racial groups. Further work has shown that, among malariaimmune Africans, HLA-B53 restricted cytotoxic T lymphocytes recognised a conserved nonamer peptide from liver-stage-specific antigen (LSA)-1 (Hill et al., 1992), suggesting a putative molecular basis for the association between HLA-B53 and resistance to severe malaria. 


\subsubsection{Immune responses directed against the liver stages of infection}

Live parasites are only directly exposed to the immune system for a short time. This occurs when sporozoites are first injected into the blood stream by mosquitoes when merozoites are first released from exoerythrocytic schizonts and between merozoite release and invasion into a new RBC during the erythrocytic cycle, processes, which occur very quickly. In addition pRBC are also taken up by cells of the immune system where parasite-derived proteins are first processed and then presented to stimulate an immune response.

The adaptive immune response against the liver stages is composed of two parts. Firstly, the invading sporozoite is extracellular and therefore is a potential target for antibody-mediated responses. It has been suggested that antibodies may block invasion of liver cells by sporozoites (Hoffman et al., 1986). However, this mechanism would only be in play after repeated infections had elicited sufficient $\mathrm{T}$ cell help for an antisporozoite mediated B cell response to develop. Secondly, as the sporozoite invades a hepatocyte and becomes intracellular, $\mathrm{T}$ cell mediated immunity is initiated (Lalvani and Hill, 1998). Mice and humans can be protected against malaria by immunisation with irradiated sporozoite (Nussenzweig et al., 1967; Clyde et al., 1973). The immune mechanism that detects sporozoite-infected hepatocytes is known to be MHC class I restricted. A study in BALB/c mice immunised with irradiated sporozoites showed that protection was dependent on the presence of cluster of differentiation (CD) $8^{+} \mathrm{T}$ cells (Schofield et al., 1987). In vivo depletion of $\mathrm{CD}^{+} \mathrm{T}$ cells abrogated protection and mice which do not express MHC class I are not protected by active immunisation or adoptive transfer of splenic $\mathrm{T}$ cells from immune mice (White et al., 1996). Cytotoxic $\mathrm{T}$ lymphocytes have also been shown to recognise infected hepatocytes and release perforin, which kills the infected cell in vitro (Weiss et al., 1990).

\subsubsection{Immune responses against blood stage malaria infection}

The malaria parasite growth cycle which causes disease occurs in the RBC. These cells have no MHC molecules on their surface or antigen processing machinery preventing direct antigen presentation to the immune system. The main site for filtration and recognition of antigens in the blood is the spleen and $\mathrm{T}$ cell responses 
against parasite antigens are elicited in the white pulp of the spleen. As MHC molecules are absent from erythrocytes, it is generally accepted that the predominant response against blood stage antigens is mediated by $\mathrm{CD} 4^{+} \mathrm{T}$ cells recognising epitopes of processed antigen displayed on antigen presenting cells (APC) that have taken up $\mathrm{pRBC}$ or free parasites.

T cell responses to Plasmodium antigens in humans are difficult to study in vivo or in vitro; therefore most studies looking at precise mechanisms of $\mathrm{T}$ cell responsiveness have been undertaken in mouse models. Blood stage immunity has been shown to be critically dependent on the presence of $\mathrm{CD}^{+} \mathrm{T}$ cells in P. chabaudi (Langhorne et al., 1989). $\mathrm{CD}^{+} \mathrm{T}$ cells mediate this protection either via a macrophage dependent mechanism or through co-stimulatory and cytokine help for malaria-specific antibody production by B cells. Malaria specific antibodies are very important, as B cell-deficient mice cannot clear a parasite infection. However, the passive transfer of immune sera alone can clear the infection demonstrating that antibodies, and not the $\mathrm{B}$ cells themselves, are required for clearance of parasites (Langhorne et al., 1998; Langhorne et al., 1990).

The cytokine, tumour necrosis factor (TNF)- $\alpha$ enhances neutrophil and monocyte/macrophage mediated phagocytosis and killing of asexual blood stages (Kumaratilake et al., 1992) and hepatic stages of $P$. falciparum (Nussler et al., 1991). The fever produced as a result of TNF- $\alpha$ release may also help limit parasite growth (Kwiatkowski, 1989). TNF- $\alpha$ production has also been shown to correlate with resistance to CM in mouse models (Stevenson and Ghadirian, 1989).

The immune response is responsible for fighting infection but can also contribute to the pathology associated with disease. Fever, produced as a result of the action of high levels of inflammatory cytokines is one example of where imbalance of immunoregulatory mechanisms can lead to pathology or protection. Evidence suggests that $\mathrm{CM}$ is linked to very high plasma levels of inflammatory cytokines, most notably the endogenous pyrogens; TNF- $\alpha$, and interleukin (IL)-1, with a positive association between TNF- $\alpha$ levels, severity of disease and the risk of death (McGuire et al., 1994). This immunopathological element to cerebral malaria has been suggested to be the response against malaria toxins (Bate et al., 1989). Malaria toxins are glycoproteins, glycolipids and other molecules released from ruptured schizonts. They have endotoxic properties and are believed to directly induce TNF- $\alpha$ by direct stimulation of unprimed 
macrophages (Kwiatkowski et al., 1989). Levels of TNF- $\alpha$ are known to be elevated in individuals who have cerebral malaria (Kwiatkowski et al., 1990). Furthermore fever was abrogated when antibodies against TNF- $\alpha$ were administered to children with cerebral malaria (Kwiatkowski et al., 1993).

Acquisition of immunity depends on the frequency of infections experienced over many years. Clinical immunity is often not reached until an individual reaches early adulthood but sterile immunity is rarely ever achieved (Marsh et al., 1989). This type of immunity, called premunition, fails to prevent the almost continuous presence of parasites in the blood during the transmission seasons and may be lost in the absence of parasites. Thus, continuous exposure to infection may actually be required in order for an effective level of immunity to be maintained.

Many antigens of malaria parasites are effective inducers of potent immune responses in the host. There are several points in the complex life cycle where the parasite is vulnerable to these immune responses. To develop to gametocytogenesis and ensure its survival, the parasite must sometimes evade host immunity and exist within the same host for extended periods. The malaria parasite has developed several ways by which to evade the host immune system and establish a chronic infection. One of the best characterised of these is antigenic variation whereby changes in antigenic identity occurs at frequencies higher than random mutation rates. In Plasmodium spp. proteins found on the RBC surface, and thus by definition exposed to the host immune system are often antigenically variant. Each new variant requires a new immune response resulting in chronic and recurrent infections and is likely to be one of the main reasons why it takes so long to develop immunity.

\subsection{Antigenic Variation}

Antigenic variation is mediated by switching expression between members of multigene families (see Section 1.5) encoding surface proteins. Antigenic variation has been well characterised for several organisms, including Trypanosomes, Babesia, Borrelia and Neisseria. 


\subsubsection{Antigenic variation in malaria}

In malaria, antigenic variation was described in several animal models before it was described in P. falciparum. The first suggestion of antigenic variability during the course of infection in the blood was in P. berghei in laboratory mice (Cox, 1962). However, antigenic variation was clearly and unequivocally demonstrated in the simian parasite P. knowlesi where a small proportion of the parasites changed the immunodominant surface polypeptide(s) on the infected erythrocyte surface during each wave of schizogony (Brown and Brown, 1965). Antigenic variation is a highly effective strategy of immune evasion. Sera from experimentally infected rhesus monkeys specifically agglutinated $\mathrm{pRBC}$ of the infecting strain but not those of other strains. Moreover, infections in the chronically infected monkeys were characterised by waves of recrudescent parasites, and that each wave was characterised by a specific antigen associated with the $\mathrm{pRBC}$ surface. The decline of each recrudescent population was accompanied by the appearance of agglutinating sera to these parasites. Brown and colleagues therefore concluded that chronic infection in malaria was maintained by serial expression of different antigenic types. Antigenic variation was subsequently shown in experiments with cloned $P$. knowlesi lines demonstrating that the ability to express successive antigenic variants was encoded within a single haploid genome (Barnwell et al., 1983a). Antigenic variation in $P$. knowlesi was attributed to proteins termed schizont-infected cell agglutination (SICA) antigens that ranged in size from $\mathrm{Mr}$ 180,000 to 210,000 (Howard et al., 1983). The SICA antigens are produced by intracellular schizonts appearing on the $\mathrm{pRBC}$ surface during late erythrocytic growth and further studies have resulted in the identification of a variant antigen gene of $205 \mathrm{kDa}$ belonging to a multigene family termed SICAvar (al-Khedery et al., 1999).

Antigenic variation has also been noted in the simian malaria $P$. fragile (Handunnetti et al., 1987), upon documentation of the ordered appearance of variant types from a single clone. In the rodent malaria P. chabaudi (McLean et al., 1982; Phillips et al., 1997), antigenic variation was demonstrated by the passive protection of naïve mice infected with parasites from a parent population of $P$. chabaudi. Pooled sera raised against the parent population was protective against parasites from the parent population but not against parasites from the recrudescent population. In the human malaria $P$. vivax, antigens were located to the surface of schizont- infected erythrocytes by an indirect immunofluorescence test using fresh unfixed infected erythrocytes from 
acute vivax malaria patients (Mendis et al., 1988). Surface immunofluorescence was detected in several isolates with some but not all the human sera, and not all sera reacted with the "positive" isolates indicating a high degree of polymorphism of the surface antigens of different $P$. vivax isolates (Mendis et al., 1988). Furthermore, sera from patients who had suffered multiple malaria attacks recognised surface antigens on more isolates than sera from patients who had experienced only one attack of malaria, indicating that repeated exposure to the disease generates antibodies against a spectrum of variants of a polymorphic malarial antigen(s). Recently a multigene family in $P$. vivax encoding variant $\mathrm{pRBC}$ surface proteins (VIR) have been described and these might be the antigenically variant proteins described on the pRBC surface (del Portillo et al., 2001).

Antigenic variation in P. falciparum was first demonstrated in vivo in squirrel monkeys using uncloned parasite lines (Hommel et al., 1983). Subsequently, it has been shown that the rate of antigenic switching is very high in vitro, occurring at around 2\% per generation (Biggs et al., 1992; Roberts et al., 1992). The protein responsible for this is the variant surface antigen $P$.falciparum erythrocyte membrane protein (PfEMP)-1 encoded by the var gene family (one of four multigene families) in P. falciparum (See Section 1.5) (Baruch et al., 1995; Smith et al., 1995; Su et al., 1995).

\subsubsection{Antigenic variation and the immune response in malaria}

In order to survive, metabolise and replicate within the host $\mathrm{RBC}$, the parasite must modify the host RBC membrane. The exposure of a parasite-encoded polypeptide on the RBC surface makes the infected cell vulnerable to immune effector mechanisms. Clonal antigenic variation is a classic method for immune evasion and the maintenance of chronic infections. As mentioned earlier, the acquired immune response to malaria is poorly understood but several studies have demonstrated the importance of antibodies in the protective immune response.

As discussed above, protective antibodies to antigenically variant proteins were observed in the $P$. knowlesi/ rhesus monkey model. Protective opsonising antibody to the infected RBC surface was induced in chronically infected monkeys in response to each new variant (Brown and Hills, 1974). 
In $P$. falciparum infections, antibodies to any particular PfEMP-1 variant appear to develop readily and to agglutinate $\mathrm{pRBC}$ in a strain-specific manner. To determine if antibody responses were protective, Marsh and colleagues carried out longitudinal studies where blood was taken from children at a point in time and then the cohort was followed for episodes of clinical malaria over a fixed period. They demonstrated that the best predictor of protection was the level of pre-existing antibody to antigens on the pRBC surface (Marsh et al., 1989). Recently, Bull and colleagues have done a large study involving several thousand Kenyan children. They have demonstrated that antiPfEMP-1 antibodies agglutinate infected erythrocytes in a variant-specific manner and that the PfEMP-1 variants expressed during episodes of clinical malaria were less likely to be recognised by the corresponding child's own pre-existing antibody response than by that of children of the same age from the same community. In contrast, a heterologous parasite isolate was just as likely to be recognised (Bull et al., 1998). These observations appear to suggest that antibody against variant antigens is a significant component of the host-protective response in both humans and animal models. In $P$. falciparum, in particular, it suggests that control of infection is dependent on the response to PfEMP-1 and that immunity to malaria depends, at least in part, to the accumulation of immunity to a range of variants.

\subsubsection{Antigenic variation in other microorganisms}

The success of establishing an infection is the ability to counter host immune responses, and antigenic variation as a mechanism of immune evasion has been utilised by several other pathogens.

One of the best-studied mechanisms of antigenic variation is that of the African trypanosome, the causative agent of sleeping sickness. Trypanosoma brucei, which multiplies extracellularly in the bloodstream, where it is directly exposed to the host immune response, produces fluctuating waves of parasitaemia. These patterns of parasitaemia are typically observed in response to the antigenically variant surface coat protein, variant surface glycoprotein (VSG) (Reviewed in (Barry et al., 1998) (Borst and Ulbert, 2001). Each VSG coat is encoded by one of the approximately 1000 different VSG genes but only a single VSG gene is expressed at a time. In order to be transcribed, VSG genes need to be located in a specialised telomeric environment, which is known as a bloodstream expression site (BES) (reviewed in Vanhamme et al., 
(2001)). One mechanism of VSG gene switching into these sites involves DNA recombination whereby a silent VSG gene is copied via gene conversion into an active expression site (Rudenko, 1999). The other mechanism is in situ switching whereby the previously transcribed BES is inactivated and a new BES is activated (Barry et al., 1998).

The asexual stages of Babesia bovis, a parasitic protozoan of cattle, cause acute disease and those animals surviving the acute disease remain chronically infected (Allred et al., 1994). Despite host immunity against this parasite, B. bovis persists in part through rapid antigenic variation of the polymorphic variant erythrocyte antigen 1 (VESA1). The gene (ves $1 \alpha$ ) encoding this protein has recently been characterised and is a member of the ves multigene family (Allred et al., 2000).

Antigenic variation has also been described in bacteria. The pili of Neisseria meningitidis and N. gonorrhoeae are composed of filamentous proteins composed of repeating subunits (pilins). Mature pili mediate adherence to a number of different eukaryotic cell types and affect interactions with epithelia, endothelia and phagocytes. Analysis of different pilin sequences has shown that they comprise of constant and hypervariable regions (Perry et al., 1987). The functional role of this heterogeneity stems from the important role of these molecules in mediating adherence to host cells. Variations in pilins influence the degree of binding as well as the absolute capacity of the bacterium to bind to host cells. The spirochete Borrelia hermsii is responsible for a relapsing fever that is characterised by a recurrent febrile attacks separated by asymptomatic intervals. An abundant outer membrane protein called variable major protein (VMP) is the primary variant antigen recognised in infection. The serotype specificity of each bacterium is thought to result from a single form of this protein that is expressed from a family of VMP genes in the organism (Deitsch et al., 1997).

\subsection{Multigene families in malaria}

Due to the several problems associated with the control of malaria, a large number of Plasmodium genome sequencing projects are currently underway. The Malaria Genome Sequencing Consortium was established to sequence the entire genome of $P$. falciparum as a collaborative venture. The sequencing of different Plasmodium species' genomes has revealed several multigene families including some already 
known or suspected. In this study multigene families have been defined as a family of genes comprising 20 or more members with a similar overall function. Multigene families make up a significant proportion of the malarial genome, e.g. in $P$. falciparum there are four multigene families, constituting more than $5 \%$ of the parasite genome, and this indicates they have an important role in parasite survival.

\subsubsection{Pf60}

This $P$. falciparum multigene family was the first to be described in human malaria parasites (Carcy et al., 1994). There are about 90 copies of $P f 60$ per haploid genome and they are located subtelomerically (Figure 1.3). The malaria genome project indicates that the majority of the $P f 60$ sequences appear to be pseudogenes because they contain frame shifts or stop codons. However, one gene (6.1) has been completely characterised (Bischoff et al., 2000) and is expressed by read through of an internal stop codon. The 6.1 gene is constitutively expressed by all mature blood stages and codes for a protein located within the nucleus. The gene consists of a seven exon, $5^{\prime}$ domain separated by an internal stop codon from a $3^{\prime}$ domain that presents a high homology with var exon II (Bonnefoy et al., 1997). This translational frameshift mechanism has only been recently described in P. falciparum, and it is yet to be determined if other genes within this family are similarly expressed. Although no function as been ascribed to the 6.1 protein, evidence for coiled coil-mediated protein interactions exists, suggesting a role in regulation of homo-or heteromeric interactions.

\subsection{2 var}

The var (variant) multigene family found in P. falciparum is probably the best characterised multigene family in Plasmodium. Approximately 40-50 copies of the var multigene family are found per haploid genome (Kyes et al., 2001). The var genes vary in size from between 6-15 kb However, all the genes share a similar gene structure with a large 5' exon coding for a large polymorphic extracellular domain and the shorter 3' exon coding for a more acidic cytoplasmic tail (Baruch et al., 1995; Su et al., 1995). The protein encoded for by this gene family is called PfEMP-1 ( $\mathrm{Su}$ et al., 1995). It was first characterised as an antigenically highly variable, high molecular weight protein $(200-350 \mathrm{kDa})$ that is readily iodinated on the surface of infected RBC. 
PfEMP-1 is detergent insoluble, demonstrating a link to the red cell cytoskeleton, and is highly sensitive to proteases (Leech et al., 1984). The true function of PfEMP-1 is not yet known but several roles have been attributed to this protein, most notably cytoadhesion (Section 1.6.2).

The majority of the molecule encoded for by exon 1 of var genes, seems to be exposed on the erythrocyte surface and has been implicated in host-cell receptor binding. The first var exon can be defined into domains called Duffy binding like (DBL) based on their homology to the $P$. vivax protein responsible for binding to the Duffy blood group antigen (Adams et al., 1992). Several malaria proteins containing these DBL motifs have now been identified and are clearly recognisable as a superfamily by virtue of the conserved spacing of cysteine residues and the presence of hydrophobic amino acids at certain positions, but there is little primary sequence conservation between them (Newbold et al., 1997a).

The DBL domains of PfEMP-1 can be divided into subfamilies of homology where five different homology groups, termed $\alpha-\varepsilon$, can be identified by sequence alignment (Smith et al., 1998). Numbering from the $5^{\prime}$ end of the gene specifies the order in which the domain occurs within the molecule. The first DBL domain is the most conserved between the different var genes and is always of the $\alpha$ type. Following this domain, the subsequent DBL domains can occur in any order and are much less conserved. They are separated by extremely divergent interdomain sequences. Interspersing DBL1 and DBL2 is the cysteine-rich interdomain region (CIDR) also found in all var genes. This domain also exists in at least 3 different sequence types, denoted $\alpha, \beta$ and $\gamma$. Thus, exon 1 codes for 2-5 DBL domains and a CIDR interspersed between DBL1 and DBL2. Apart from short conserved sequence motifs in the first DBL domain, exon 1 displays considerable sequence diversity. The $3^{\prime}$ exon encodes a conserved acidic region which is low in cysteine residues ( $\mathrm{Su}$ et al., 1995). Initial analyses using 2D chromosome blots and yeast artificial chromosome (YAC) library screening showed that most if not all, chromosome ends contain at least one var (Fischer et al., 1997; Rubio et al., 1996; Hernandez-Rivas et al., 1997) and the genes lie within the arrays of $P$. falciparum-specific subtelomeric repeat sequences. The $P$. falciparum genome project has allowed the accurate organisation of the var genes (chromosomes 2 and 3)(Bowman et al., 1999; Gardner et al., 1998). The var genes are distributed on all 14 chromosomes but are predominantly located within subtelomeric 
Consensus

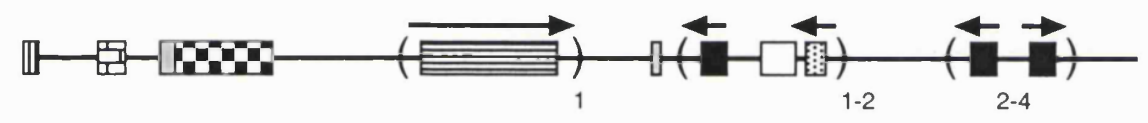

\begin{tabular}{|c|c|c|}
\hline rif & Pf60 & stevor \\
\hline Telomere & 场R-CG7 & Rep11 \\
\hline
\end{tabular}

Figure 1.3 A consensus for the arrangement of repetitive DNA sequences and multigene families on P. falciparum telomeres based on the telomeres of chromosomes 2 and 3. The relative numbers and orientation are conserved for members of the multigene families. The open reading frames lie within ordered telomere repeat sequences. The arrows indicate orientation of open reading frames. Modified from Bowman et al., (1999) and Kyes et al.,(2001). 
domains. They appear to be located a consistent distance from the telomere, and the most telomeric gene is always transcribed toward the centromere (Figure 1.3). Chromosomes, 4, 7, 8 and 12 contain var genes distributed within internal clusters and this distribution is conserved across different parasite isolates (Rubio et al., 1996; Thompson et al., 1997). Furthermore, chromosomal internal var gene sequences are more conserved and therefore more closely related to those of other parasite clones than are their subtelomeric equivalents.

The different var genes vary in sequence and this results in antigenic heterogeneity and variability in binding specificity. It should be emphasised that different parasite isolates contain not only different numbers of var genes located in varying genomic locations but also different repertories of sequences. Thus the total number of distinct var gene sequences is in the order of thousands.

\subsection{3 rif}

The repetitive interspersed family (rif) was originally characterised as multi-copy $P$. falciparum sequences but were not studied further due to their apparent lack of a translation start site (Weber, 1988). However, sequence analysis arising from the genome project revealed a spliced exon, $\sim 300 \mathrm{bp}$ upstream of each rif, that encoded a translational start site and a putative secretory signal sequence (Cheng et al., 1998). Further analysis has revealed 17 rif genes on chromosome 2 and, by extrapolation of sequence data from chromosomes 2 and 3, (the sequences of which are complete) it has been suggested that there are in excess of 200 rif copies per haploid genome (Kyes et al., 1999). Like var, the rif genes are located in close association to each other in clusters within $50 \mathrm{~kb}$ of the telomeres (Figure 1.3) and have subsequently been located on all chromosomes (Cheng et al., 1998). Rif genes have 2 exons; one short exon, followed by an intron of $\sim 200 \mathrm{bp}$ and a second exon of variable length around $1 \mathrm{~kb}$ (Cheng et al., 1998). The products of rif genes (RIFINs) consist of a highly conserved $\mathrm{N}$ terminal sequence (exon 1) with some characteristics of a signal peptide. In addition, two predicted putative transmembrane domains flank the highly polymorphic region of exon 2. Protein structure predictions strongly indicate that RIFINs are membrane bound proteins with the C-terminal end in the cytoplasm and the polymorphic region exposed on the outside (Cheng et al., 1998). Kyes et al., (1999) have shown that 
RIFINs are clonally variant proteins of $35-44 \mathrm{kDa}$ expressed on the surface of infected RBCs and are primarily transcribed at the late ring/early trophozoite stage (18-23 h). RIFINs are immunogenic in natural infections as individual strains are specifically recognised when products of surface-labelled $\mathrm{pRBC}$ are immunopreciptated by human immune sera (Fernandez et al., 1999; Kyes et al., 1999). Although no definitive function has been ascribed to members of this family, there is some experimental evidence that RIFINs are thought to play an accessory role in rosetting and CD31 binding (Fernandez et al., 1999; Kyes et al., 1999).

\subsubsection{Stevor}

Subtelomeric variable open reading frame (stevor) was first reported as $7 \mathrm{~h} 8$ and was identified as an expressed sequence detected by a monoclonal antibody in $P$. falciparum (Limpaiboon et al., 1991). Like rif genes, stevor genes are also located subtelomerically on all chromosomes (Figure 1.3), are $\sim 1 \mathrm{~kb}$ in length and are very similar to rif in both gene structure and protein structure predictions (Figure 1.4), such that originally they were mistaken for the same family. However, the genome sequencing project has demonstrated that the stevor genes are more conserved, are slightly shorter than rif genes, and have a reduced copy number (around 34 copies per haploid genome family) (Cheng et al., 1998).

\subsubsection{Py235}

The py 235 multigene family of the rodent malaria $P$. yoelii codes for a $235 \mathrm{kDa}$ rhoptry protein (p235). The py235 family consists of at least 11 members (Borre et al., 1995; Keen et al., 1990) with observations in our laboratory indicating that there are up to 35 copies per genome (Khan et al., unpublished). Although these genes are found subteleomerically they are not located on all chromososmes (Owen et al., 1999). Even though this protein is not located on the RBC surface, it is speculated to mediate merozoite antigenic and phenotypic variation by facilitating the ability of the merozoite to invade RBC of different ages. This was demonstrated when monoclonal antibodies against p235 were passively transferred to mice. On subsequent infection of these mice with the virulent $P$. yoelii strain YM, which infects all RBC, the invasive properties of the parasite had changed to that of the avirulent $17 \mathrm{X}$ strain which preferentially invades 


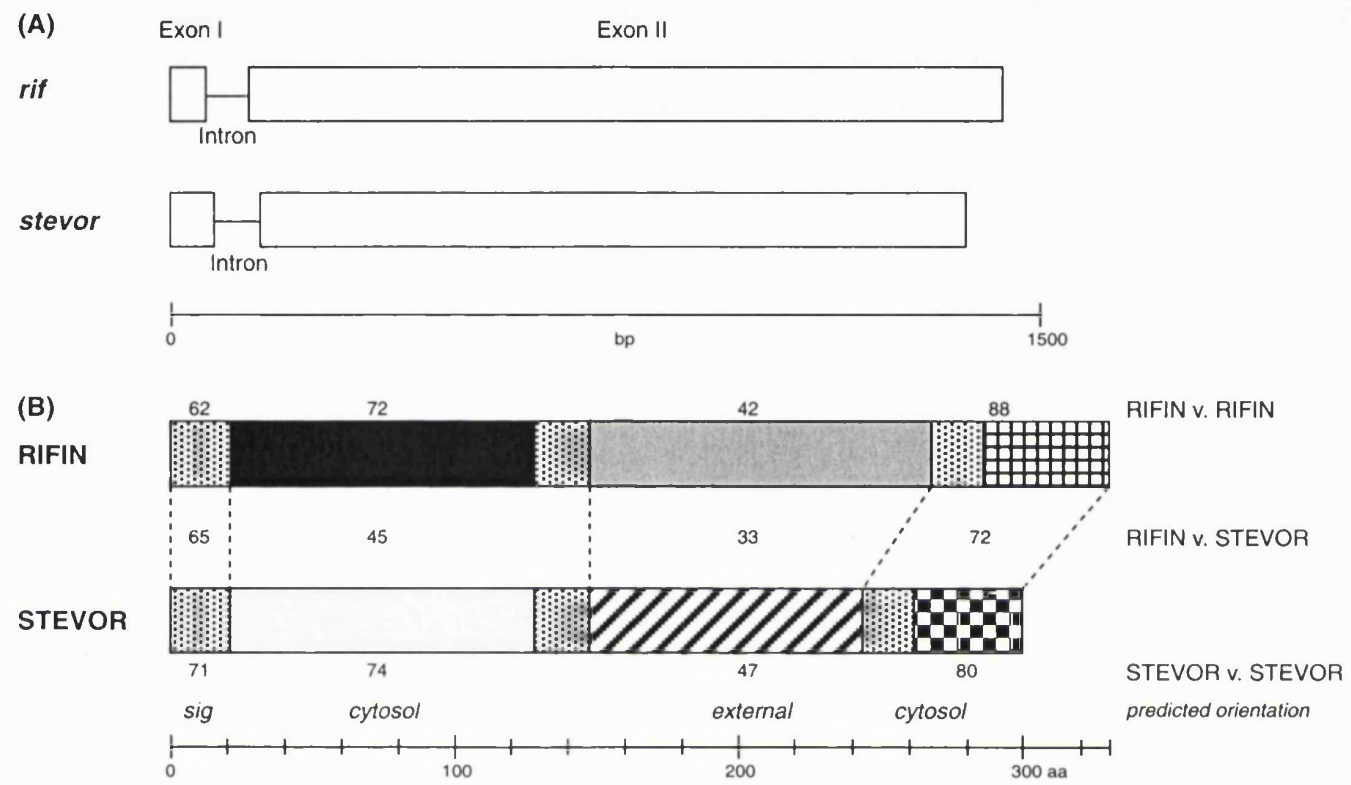

Figure 1.4 A schematic comparing stevor and rif. (A) Both rif and stevor are similar in gene structure, with a short first exon, and intron of $\sim 300 \mathrm{bp}$ and a second exon of $~$ $1 \mathrm{~kb}$ (stevor) or $\sim 1.3 \mathrm{~kb}$ (rif). (B) STEVOR and RIFIN-predicted protein sequences are structurally similar. Putative transmembrane domains are marked with dots. Exon 1 encodes a potential signal sequence (sig.) and, Exon II encodes a putative C-terminal transmembrane region followed by a short cytoplasmic tail. Percent similarity between sequence pairs is given (numbers above each region compared), for RIFIN versus RIFIN, STEVOR versus RIFIN and STEVOR versus STEVOR. For both predicted sequence types extreme diversity lies in the region predicted as being exposed outside the cell membrane. Modified from Kyes et al., (2001). 
reticulocytes (Freeman et al., 1980; Holder and Freeman, 1981; Oka, 1984). In addition to playing a role in host cell selection it has been suggested that this gene family may also be involved in immune evasion. The merozoite is exposed to the immune system after schizont rupture and it would be of an advantage to ensure that some merozoites evade the immune response and successfully invade RBC.

Py235 is distributed throughout the genomes of both the $\mathrm{YM}$ and $17 \mathrm{X}$ lines of P. yoelii and like var appear to be located close to telomeres (Owen et al., 1999). By contrast to var, a different method of transcriptional control appears to be utilised in the py235 multigene family. Preiser and Jarra, (1998), have been able to distinguish several subfamilies of py235 and have demonstrated that the virulent line does not transcribe its entire py 235 gene repertoire. They have further shown that a single schizont expresses multiple members of py235 whereas an individual merozoite transcribes only one (Preiser et al., 1999). This suggests that transcription of one member of the gene family is initiated at the late trophozoite stage and, as the parasite matures to form a multinucleated schizont, further py235 become transcriptionally active. A mechanism like this may give the parasite a further advantage in establishing a chronic infection.

\subsection{6 vir}

The most recently identified multigene family, analagous to rif, is that of the $P$. vivax variant genes (vir). There are thought to be between 600-1000 vir copies per haploid genome and they are also located subtelomerically. The vir genes have three exons: a short first exon, lacking a consensus signal peptide sequence; a longer second exon containing a predicted transmembrane domain at its $3^{\prime}$ end; and a third exon with a highly conserved region. Homologues of the vir family have been found in the rodent malarias $P$. yoelii and P. chabaudi (Dr. P Preiser, pers comm.). Vir genes are expressed during intra-erythrocytic development and encode proteins (VIR) that are immunovariant in natural infections. VIR proteins are $\sim 36 \mathrm{kDa}$, and share no homology to known proteins in BLAST analysis (del Portillo et al., 2001).

\subsubsection{Genomic location of multigene families leads to an increase in the repertoire of genes}


The close proximity of the multigene families to each other and their location at the subtelomeric ends of the chromosomes may be advantageous to the parasite. The location of a large proportion of these genes within telomeric repeat sequences may be necessary to promote recombination between genes and thus the continued generation of diversity.

Genes located at telomeric ends are often part of specialised higher order nuclear architecture such as the chromosome end cluster at the periphery of nuclei as demonstrated by the clustering of $P$. falciparum chromosome ends (Freitas-Junior et al., 2000). This may be a significant element in promoting ectopic exchange of sequences in the subtelomeric regions of $P$. falciparum. Telomere mediated enhancement of recombination would perhaps augment the effect of the relatively small var gene complement in individual parasites during chronic asexual blood stage infection. It has been calculated that recombination between telomeric var genes is some eightfold higher than that measured in the genome as a whole (Taylor et al., 2000a).

Antigenic diversity of these multigene families is also another way of increasing the repertoire of genes. Antigenic diversity can be defined as the expression of antigenically different forms of parasite gene products by parasites of different genotype within any given isolate. This heterogeneity is maintained by genetic recombination, in addition to mutation (Walliker et al., 1975; Walliker et al., 1987). The malaria parasite exists as a series of genetically and biologically distinct species and lines. Isolates collected in the wild from the natural host may represent complex mixtures of different lines and/or species of parasites (Thaithong et al., 1984). Analysis of cloned parasite lines, derived from such islolates, has allowed the recognition and definitive characterisation of parasite biodiversity. As a result of intensive studies on the comparison of DNA and amino acid sequences from well characterised parasite genes and gene products from different parasite isolates, antigenic diversity has been demonstrated not only multigene families, but also in many different antigens from different parasite stages. These include the CSP in mature sporozoites (Jongwutiwes $e t$ al., 1994) merozoite surface antigens-MSP-1, MSP-2 and MSP-3 (Miller et al., 1993) (Farnert et al., 1997) (McColl and Anders, 1997), rhoptry antigens (Howard et al., $1986)$ in the erythrocytic stage and gamete/gametocyte antigens and zygote/ookinete antigens in sexual stages (Foo et al., 1991). The diversification of antigens may confer on the parasite the ability to infect a host, which has previously only been exposed to 
parasites of a different genotype, as the immunity directed at these parasites is species and strain specific.

\subsection{The var multigene family and its products}

There is still relatively little known about Plasmodium multigene families themselves. The mechanisms of transcriptional control are yet to be established and the exact function of the proteins encoded by these families is unknown. The best characterised of these families is the var multigene family and its products. The functions attributed to this family further highlight the importance of multigene families both for parasite survival and in understanding the pathology associated with disease.

\subsubsection{Transcriptional control of var}

The transcription of var genes is stage specific within the erythrocytic cycle. A high level of transcripts can be detected by Northern blot between 3 and $18 \mathrm{~h}$ posterythrocyte invasion and this quickly declines to undetectable levels (Chen et al., 1998b; Kyes et al., 2000). However, despite transcription of var genes early within the asexual cycle, the gene product is not detected at the RBC surface until around $16 \mathrm{~h}$ post invasion (Kyes et al., 2001). This is likely to be partly due to the time it takes for the parasite to assemble the machinery necessary to transport the protein across the parasite and parasitophorous vacuole membranes (PVM), across the cytoplasm and into the red cell membrane (Section 1.7).

It appears that there are no defined var expression sites, like there are for VSG genes, and all var genes can be expressed irrespective of their chromosomal location (Fischer et al., 1997; Hernandez-Rivas et al., 1997). Some groups have suggested that ubiquitous transcription of all var gene variants appears to occur in early ring stages but is tightly regulated in trophozoites (Chen et al., 1998b). Further investigation has suggested that the promiscuous transcription of var during early ring stages results in only one full length transcript while the rest are terminated early (Taylor et al., 2000a). Noviyanti et al., (2001) have suggested that several full-length var transcripts can be detected at trophozoite stages but only one dominant transcript is expressed. From studies correlating var gene expression with antigenic and adhesive properties of 
infected erythrocytes, it appears that only a limited number of, probably only one, var gene variant is expressed on the $\mathrm{pRBC}$ surface with var gene switching occurring at a rate of $\sim 2 \%$ per generation in vitro (Roberts et al., 1992; Scherf et al., 1998; Smith et al., 1995).

Although the mechanistic details of var gene switching still remain to be elucidated, initial data have suggested that this mechanism is fundamentally different from that of VSG switching in African trypanosomes (section 1.4.3). Thus, var gene expression does not occur from a unique expression site as expressed var genes were mapped to several different chromosomal loci (Fischer et al., 1997; Hernandez-Rivas et al., 1997; Su et al., 1995). Investigations on the nature of var transcription were carried out in parasite clones serially panned over cells expressing different adhesion molecules. Parasite populations with defined adhesive phenotypes for intracellular adhesion molecule (ICAM)-1, chondroitin sulphate A (CSA) or CD36 receptors expressed a single and distinct var variant (Scherf et al., 1998). The chromosomal location of each var variant remained unchanged irrespective of its transcriptional status and appeared to occur in situ with no requirement for the transposition of the var gene to a transcriptionally active site. Finally, regulation of var expression appears to be mediated at the transcriptional level as demonstrated by nuclear run on anlayses, suggesting that while one promoter is activated the others are silenced (Scherf et al., 1998). Transcriptional silencing can be overcome by changes in chromatin states through reversible histone acetylation which has been described in blood stages of $P$. falciparum (Darkin-Rattray et al., 1996; Wolffe, 1996).

The mechanisms underlying chromosomal switching in P. falciparum are yet to be elucidated. The modification of nucleotides can play a significant role in the control of gene expression as demonstrated in African trypanosomes (Gommers-Ampt et al., 1993) and there is evidence for DNA methylation in P. falciparum (Pollack et al., 1991). However, experiments performed to investigate this suggest that changes in the methylation pattern or nucleosomal phasing do not play a role in var gene switching (Scherf et al., 1998). The role of trans-acting factors in controlling var transcription has been examined through the use of transient transfection experiments by Deitsch and colleagues. They showed that silent var promoters become transcriptionally active when removed from their chromosomal context suggesting that switching is not mediated by trans-acting factors alone. Recently, this group has demonstrated that the 
conserved intron sequence (which separates the two var exons) is responsible for the silencing of its promoter, and that this silencing mechanism is specific and occurs by co-operative action with the upstream var 5' flanking region. This silencing was S-phase dependent and, indicates that co-operative DNA binding complexes and changes in chromatin structure are involved in the control of transcription (DarkinRattray et al., 1996; Wolffe, 1996). It is not known whether transmission through the insect stage acts to reset the repertoire to express a particular var gene, as has been suggested for trypanosomes.

\subsubsection{PfEMP-1, its role in virulence and putative functions}

In addition to acting as a means of immune evasion, PfEMP-1 is also an important virulence factor because it mediates adhesion to a variety of host cell types. The most important adhesive interaction of infected red cells is the phenomenon of sequestration in which $\mathrm{pRBC}$ adhere to endothelial cells lining the microvasculature protecting $\mathrm{pRBCs}$ from entrapment and loss in the spleen. As a result of sequestration, cells infected by mature P.falciparum parasites are absent from the peripheral circulation. Although the property of cytoadherence is universal, under some conditions the parasites appear to accumulate in particular organs. Another property of P. falciparum infected RBC, also mediated by PfEMP-1, is rosetting; the ability of $\mathrm{pRBC}$ to adhere to uninfected RBC. Sequestration and rosetting result in the obstruction of blood vessels, comprimising blood flow to the affected organs and this is considered the primary cause of CM (Berendt, 1993). P. vivax on the other hand does not sequester nor cause CM lending weight to the importance of these events in this aspect of the pathology of the falciparum disease. P. falciparum is also a major cause of maternal death, abortion, still-birth, premature delivery and low birth-weight in endemic areas (Menendez, 1995). The placenta is a preferred site for sequestration of $\mathrm{pRBC}$ resulting in increased susceptibility of pregnant women to malaria. Thus, the pathology associated with $P$. falciparum infections is largely believed to be due to the adhesive properties of the infected erythrocytes. It is important to mention that anaemia also contributes significantly to the severe disease associated with malaria and is reviewed in (Menendez et al., 2000).

Early speculation that DBL regions may be involved in adhesion has been supported by recent results mapping the binding sites for complement receptor 1 (CR1), 
ICAM-1, CSA, CD31 to different DBLs (Rowe et al., 2000; Smith et al., 2000; Buffet et al., 1999; Reeder et al., 1999; Chen et al., 2000). Combinations of specific DBLs determine within expressed PfEMP-1 which receptors the parasite can bind to (Peterson et al., 1995). Mapping of the specific receptor binding sites to motifs within PfEMP-1 show that these tend to cluster around a N-terminal 'head structure' of the PfEMP-1 molecule (Su et al., 1995) including the major rosetting receptor complement receptor 1 (CR1)(Rowe et al., 1997) and the binding site for CD36 (Baruch et al., 1997).

\subsubsection{Sequestration}

The most common interaction of $\mathrm{pRBC}$ and the endothelium appears to be between PfEMP-1 and CD36 with studies suggesting that most, if not all, $P$. falciparum parasites adhere to vessels using these molecular interactions. This interaction occurs between the CIDR region of PfEMP1 and CD36 and may involve conserved structures. Exceptions to CD36-mediated binding are the CSA binding parasites found in the placenta (Buffet et al., 1999; Reeder et al., 1999). Another major receptor is ICAM-1 (Berendt et al., 1989), with a large proportion of parasite isolates able to bind to this receptor. The binding site for ICAM-1 has been mapped to the DBL2 $\beta-C 2$ region (Smith et al., 2000). ICAM-1 expression is highly upregulated on endothelium during malaria and it has been suggested that one of the causes of severe disease may be high levels of receptor linked with a large population of parasites able to bind to ICAM-1. (Newbold et al., 1997b) have shown that there is very little CD36 expression in brain endothelium and that the presence of ICAM-1 on endothelial cells lining the vessel is by far the best predictor of the presence of parasite adhesion. This suggests that increased binding to ICAM-1 is associated with cerebral malaria. However, contradictory evidence exists, and more recently it has been suggested that (a) the ability to bind to multiple receptors is associated with severe disease and (b) that several receptor-ligand interactions work synergistically in bringing about severe disease (Heddini et al., 2001; Rogerson et al., 1999).

Maternal malaria is a major cause of pregnancy-related complications in endemic areas (Menendez, 1995). Women that have already developed clinical immunity to malaria become symptomatic to malaria when they become pregnant. The placenta is a preferential site for sequestration; infections in the placenta often reach 
very high densities and can be observed in the absence of detectable peripheral parasitaemia. pRBC obtained from the placenta are the only ones that do not bind to CD36. Several lines of evidence suggest that CSA is the parasite receptor that mediates adhesion of pRBC to the human placenta (Fried and Duffy, 1996). Parasites isolated from the placenta show a strong tendency to adhere via CSA and appear to represent rare variant types not recognised by sera from adult men in malaria endemic areas (Fried and Duffy, 1996; Maubert et al., 1999).

It is now known that $P$. falciparum $\mathrm{pRBC}$ can adhere to vascular endothelium via a variety of host receptors. The receptors identified so far are CD36, ICAM-1, vascular cell adhesion molecule (VCAM-1), CD31, endothelial-selectin, plateletselectin, thromobospondin (TSP) and possibly the $\alpha_{\mathrm{v}} \beta_{3}$ integrin.

Most studies of cellular adhesion phenomena have focused on P. falciparum; however, sequestration has also been noted in a number of other malaria species capable of infecting hosts such as rodents and primates.

\subsubsection{Rosetting}

Binding of $\mathrm{pRBC}$ to two or more uninfected $\mathrm{RBC}$ to form so-called "rosettes" is common in field isolates and has been shown to be associated with severe disease (Rowe et al., 1995). Almost all studies have shown a correlation between rosetting and severe malaria unlike the case with cytoadherence where isolates from patients with severe malarial anaemia showed significantly reduced adhesion compared with isolates from mild and cerebral malaria cases (Newbold et al., 1997b). It has also been suggested that by causing aggregates of cells, rosetting leads to microvasculature obstruction (Kaul et al., 1991). Serum from malaria immune individuals have been shown to disrupt rosettes in vitro (Carlson et al., 1990).

Recent data indicate that rosetting is mediated by PfEMP-1 (Chen et al., 1998a; Rowe et al., 1997). By deriving rosetting and non-rosetting lines from a clone, it was shown that a single dominant var gene is expressed in rosetting but not non-rosetting organisms (Rowe et al., 1997). Rosetting appears to be a heterogeneous phenomenon in which a number of erythrocyte ligands, such as ABO blood groups (Carlson and Wahlgren, 1992), CD36 (Handunnetti et al., 1992), sulphated glycoconjugates (Rowe et al., 1994) and CR1 (Rowe et al., 1997) differentially modify the degree of rosette 
formation in different isolates. The reasons why $\mathrm{pRBC}$ form rosettes are unclear but rosetting may be protective for the parasite because it may conceal the infected cell from malaria specific antibodies and/or phagocytic cells (Wahlgren et al., 1989). It has also been suggested that rosetting may facilitate merozoite entry into uninfected cells within the rosette, but there is data to suggest that this is not the case (Clough et al., 1998).

\subsubsection{Putative functions of PfEMP-1}

Although the exact function of PfEMP-1 is unknown, several possible roles have been suggested. It has been proposed that binding to host endothelium prevents splenic clearance of mature parasites form the circulation. Cytoadhesion is not required during in vitro cultivation and may be frequently lost. Also, the ability of malaria parasites to sequester is modulated in splenectomised monkeys so that an intact spleen plays a role in the maintenance of the normal phenotype (Hommel et al., 1983). Passage of cloned parasites through splenectomised animals results in the appearance of parasite populations that no longer express PfEMP-1 on the RBC surface (Barnwell et al., 1983a; Barnwell et al., 1983b). Re-introduction of these parasites into intact animals results in the reappearance of PfEMP-1 onto the surface of the RBC in a manner by which PfEMP1 gradually reappears in the whole population. However, $P$. falciparum is the only human malaria in which all the mature parasite stages exhibit cytoadherence. This is in contrast to other species where almost all cells infected with mature forms of $P$. vivax, $P$. ovale and $P$. malariae pass through the spleen. By providing a mechanism for splenic avoidance, it is almost as if $P$. falciparum has marked itself for destruction by the host immune system.

It has been proposed that by providing a target for the immune system the parasite is able to regulate its growth so that it can generate transmissible (gametocyte) forms before killing the host (Saul, 1999). PfEMP-1 was found to mediate adhesion of early stage gametocytes and these can adhere to the same cell lines as asexual stages (Hayward et al., 1999). Gametocytes are absent from the peripheral circulation and thus, adhesion may be important not only for host immune evasion, but also for gametocytes during their development rather than solely for asexual forms. 
Finally, another possible function of PfEMP-1 is that of immunosuppression. It has been reported that children with acute P.falciparum malaria have depressed responses to a variety of vaccine antigens (eg meningococcal $\mathrm{C}$ polysaccharide, and typhoid and poliomyelitis vaccines) while responses to other antigens remain unaffected (eg S. typhi $\mathrm{H}$ antigen, measles and Bacille Calmette-Guerin vaccines (Greenwood et al., 1972; Bradley-Moore et al., 1985). Immunosuppression is likely to extend to specific responses to malarial antigen since decreased lymphocyte responsiveness to plasmodium antigens in vitro has been observed by several groups in subjects undergoing acute infection with P. falciparum (Ho et al., 1986; Riley et al., 1988; Webster et al., 1988). Until recently, the cellular basis for immunosuppression was unidentified, but recent evidence suggests that PfEMP-1 may also mediate adhesion to dendritic cells (the most potent APC), causing immune disregulation and thereby reducing the efficiency with which the host is able to mount a protective response (Urban et al., 1999; Urban et al., 2001).

\subsubsection{Other proteins associated with the pRBC surface}

As the parasite develops in the host's erythrocyte, a number of changes take place including modifications to the cell membrane to form knob-like structures, an increase in the rigidity of the cell, alterations in the metabolite transport and the insertion of a number of parasite-derived proteins into the surface of the $\mathrm{pRBC}$ membrane. Several of these proteins found at or below the surface of the infected erythrocyte are also involved in the cytoadherence properties of pRBC (Figure 1.5).

\subsubsection{KAHRP}

As well as relying on surface exposed proteins, efficient cytoadherence requires the presence of localised protrusions on the pRBC surface called knobs. The knobassociated histidine rich protein (KAHRP) is a major component of the knobs (Kilejian, 1980). PfEMP1 is transported to the red cell surface and is also a component of the knobs. With the development of techniques for transient and stable transfection of P. falciparum, it has been possible to disrupt, or 'knock out' parasite genes. A knockout of KAHRP resulted in a smooth RBC membrane surface devoid of knobs therefore demonstrating that KAHRP is essential for knob formation (Crabb et al., 
1997). Adhesive properties of the knockout line under static conditions were not affected, but under physiological flow conditions, adhesion was greatly reduced, showing that knobs play an important role in adhesion. PfEMP-3 knockout also resulted in the loss of adhesion but this was found to be indirect due to disruption of PfEMP1 transfer to the outer erythrocyte surface (Waterkeyn et al., 2000).

\subsubsection{CLAG}

In the early 1990s it was noted that some laboratory -adapted isolates had lost the ability to cytoadhere, measured by binding to C32 melanomas, and this phenotypic loss associated with a subtelomeric deletion on chromosome 9 (Shirley et al., 1990). A detailed study of this region defined a candidate cytoadherence-linked asexual gene (clag 9) that was transcribed in asexual stages and consisted of nine exons (Craig, 2000). Genetic knockout of this locus resulted in a loss of CD36 binding phenotype (Trenholme et al., 2000). The precise subcellular location of this protein is still debatable and it is possible that clag 9 produces its effect on cytoadherence indirectly through enabling PfEMP-1 transport rather than interacting directly with a host receptor (Trenholme et al., 2000).

\subsubsection{Modified Band 3}

Band 3 is a highly abundant $\mathrm{RBC}$ host protein that acts as an anion transporter for the erythrocyte. Cryptic epitopes of this protein are exposed in ageing RBCs and play an important role in mechanisms for the removal of senescent cells. Infections by $P$. falciparum also causes structural modifications to band 3 that can be recognised by specific monoclonal antibodies (mAbs) that also inhibit cytoadherence to C32 melanomas (Winograd and Sherman, 1989; Crandall and Sherman, 1991). Regions of band 3 that are involved in this interaction have been identified (Crandall and Sherman, 1996). Injection of a peptide corresponding to one of these regions into $P$. falciparum infected saimiri monkeys resulted in the release of mature erythrocytic stage forms into the peripheral circulation. The molecular basis for this interaction is unclear but data suggest that modified band 3 may bind to TSP (Lucas and Sherman, 1998). 


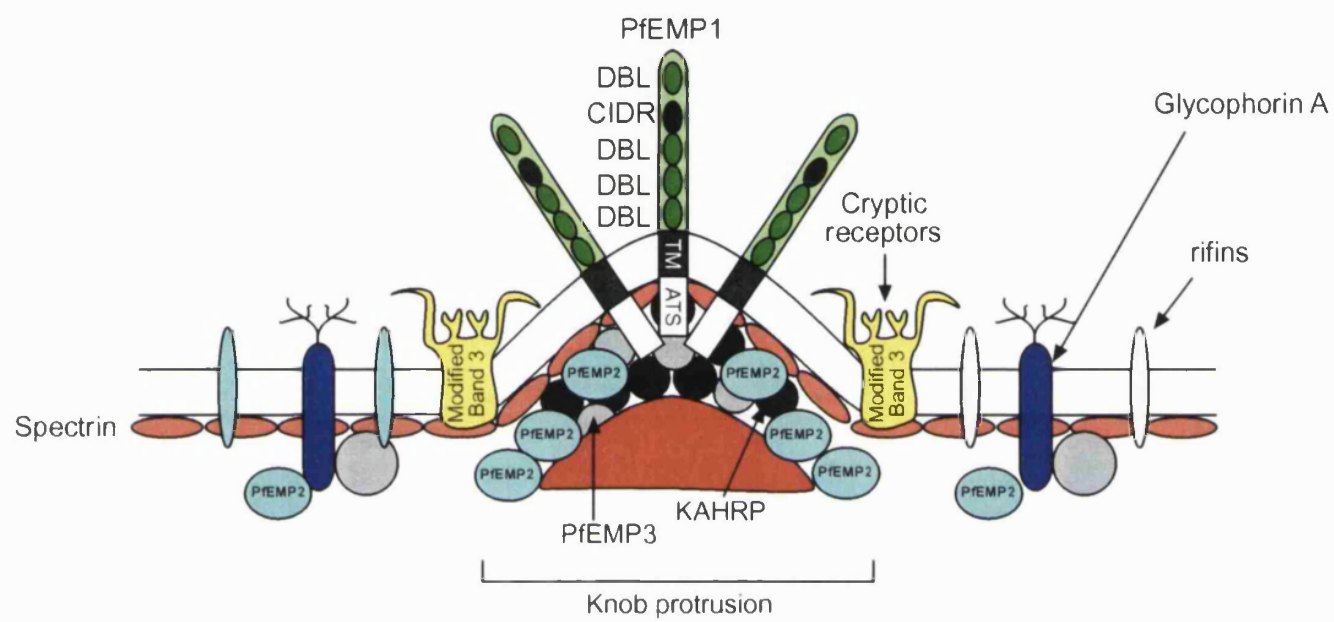

Figure 1.5 Schematic representation of the membrane skeleton of a $P$ falciparum infected RBC membrane to show alterations associated with the $\mathrm{pRBC}$ membrane and proteins believed to be associated with adhesion. Localisation of PfEMP-1 into knobs might occur by association with KAHRP a major and essential component of knobs. Receptors in cryptic regions of band 3 become exposed at the surface with the intracellular growth of the parasite inside the RBC. Each PfEMP-1 molecule consists of Duffy binding-like (DBL) domains, a cysteine-rich interdomain region (CIDR), a transmembrane (TM) domain and an acidic terminal segment (ATS). The other parasite protein known to be on the surface is the rifin. Other proteins thought to be associated with adherence and present at the knob region are P. falciparum erythrocyte membrane protein (PfEMP)-2 and PfEMP-3. 


\subsubsection{Others}

Recently a new form of cytoadherence has been described involving the immature ring-stage forms of pRBC (Pouvelle et al., 2000). Mature parasites selected for adhesion via CSA show an associated binding to cultured endothelium and placenta but not via CSA. This is mediated by parasite-encoded proteins called ring surface protein $1(\mathrm{RSP}-1)$ and 2 on the surface of the $\mathrm{pRBC}$ that are expressed shortly after erythrocyte invasion.

Another parasite adhesin is sequestrin which is a $207 \mathrm{kDa}$ protein accessible during suface labelling experiments, $\mathrm{pRBC}$ specific and structurally conserved across several isolates (Ockenhouse et al., 1991).

\subsection{Transport of proteins to the erythrocyte membrane of $\mathrm{pRBC}$}

It is clear that for the parasite to survive within the host several modifications are made to the host RBC. However, the host RBC lacks the machinery necessary for the transport of proteins and thus parasite-induced modifications are made for proteins to be trafficked to the RBC membrane. The classical secretory pathway in eukaryotic cells involves the transport of proteins between intracellular organelles by the budding and fusion of small vesicles. Exported proteins transit through the endoplasmic reticulum (ER) and the Golgi apparatus, prior to release, at the cell surface. Protein trafficking in malaria-infected erythrocytes has an added level of complexity in that to target proteins to the $\mathrm{RBC}$ membrane, the parasite has to transport them past its own plasma membrane (PM) across the parasitophorous vacuolar membrane (PVM) and then out to the RBC membrane (Figure1.6).

Elements of the classical vesicle-mediated secretory pathway for the export of proteins are present within the cytoplasm of the malaria parasite and appear to be involved in the transport of proteins to the PM. Homologues of a number of trafficking components have been found including the ER molecular chaperones Pfgrp (Kumar et al., 1991) and PfERD2 (Elmendorf and Haldar, 1993) homologues of the KDELbinding protein. Moreover, brefeldin A, a drug that specifically blocks vesiclemediated trafficking, blocks the secretion of some proteins (Benting et al., 1994; 
Hinterberg et al., 1994). However, a morphologically identifiable Golgi has not been observed in trophozoite stage parasites although tubular membranous elements have been identified that might be a Golgi apparatus (Kirk et al., 1999). An alternative pathway of trafficking proteins, which is not inhibited by brefeldin $\mathrm{A}$ has also been described (Mattei et al., 1999) (Figure 1.6).

Very little is known about the trafficking machinery in the erythrocyte cytosol. Ultra-structural studies revealed a variety of membranous structures in the RBC cytoplasm that may be involved in protein trafficking (Elford et al., 1995). The most prominent structure is the tubovesicular network (TVN) which appears to be a series of blind "appendices" extending out from and wholly connected to the PVM. In addition to the TVN, at least three types of membranous structures have been observed in the cytoplasm of pRBC. These are the Maurer's Clefts (MC), membranous whorls, and small single membrane-bound vesicles. These structures are believed to deliver and possibly assemble complexes of proteins associated with or destined for the RBC membrane/surface (Albano et al., 1999a; Waterkeyn et al., 2000) (Figure 1.6).

A detailed description of protein trafficking in malaria is beyond the scope of this introduction and is covered in several reviews (Albano et al., 1999b; Foley and Tilley, 1998). 


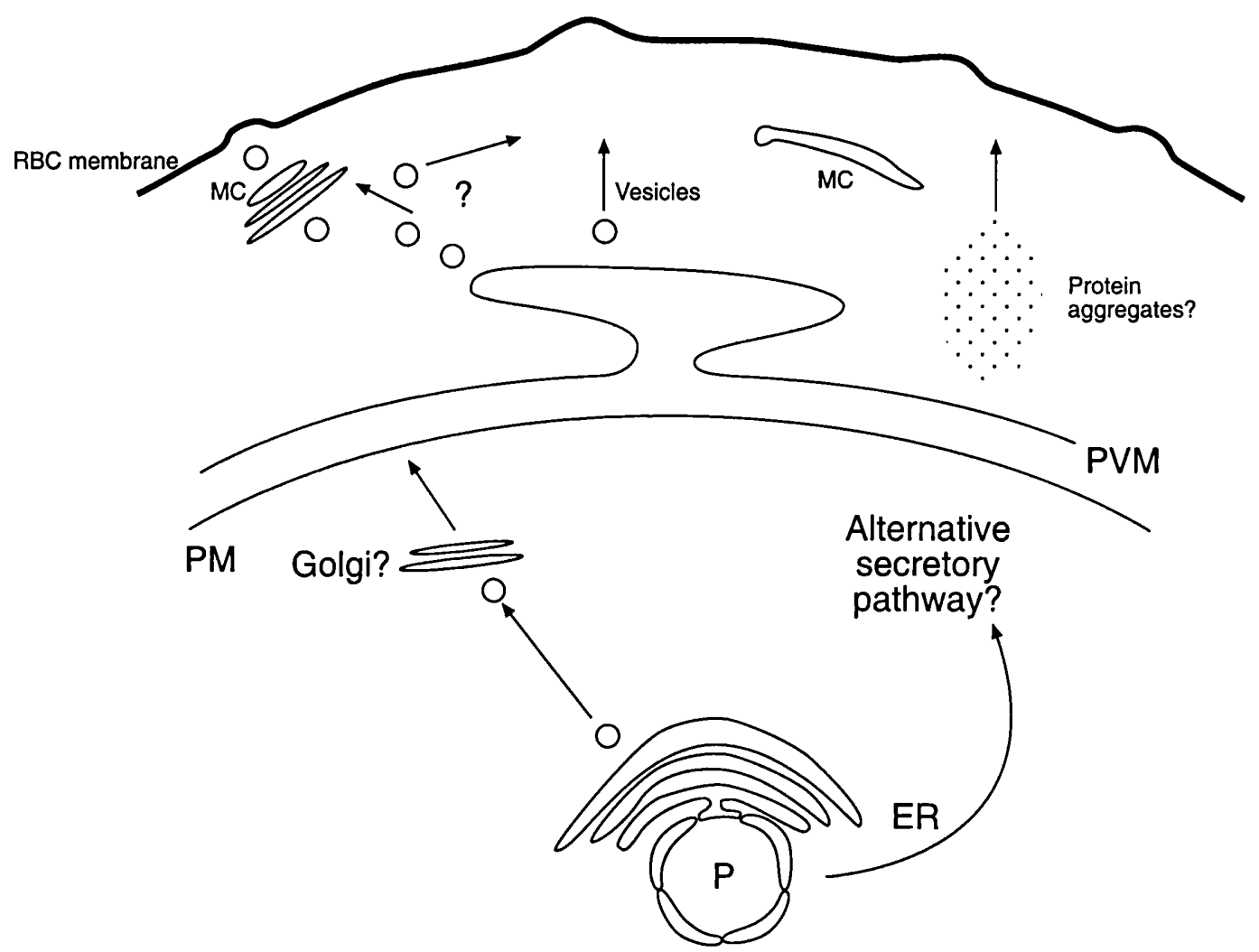

Figure 1.6 Schematic of putative trafficking pathways in malaria-infected erythrocytes. The parasite resides in the parisotophorous vacuole within the erythrocyte. Unlike other erykaryotic cells, the parasite has to secret proteins beyond the confines of its own plasma membrane into the parasitophorous vacuolar membrane (PVM), tubovesicular network (TVN) and erythrocyte cytosol and membrane. Parasites may utilise a classical or an alternative secretory pathway for traffic to the parasite membrane parasitophorous vacuole (PV). Transit of proteins from the PV to the RBC membrane occurs by a largely uncharacterised pathway. 


\subsection{Project Aims}

Plasmodium species have several multigene families, highlighting their importance in parasite survival. Investigations of these families have elucidated their role in parasite immune evasion, virulence and host pathology. Combinations of the roles of these proteins, including antigenic variation, rosetting and parasite sequestration contribute on one hand to the survival of the parasite and on the other are intrinsically linked to pathology. Antigenic variation and adhesion mediated by the same molecule (PfEMP-1), ensures maximal survival and propagation of the parasite. The significance of these adhesive events and the primary function of the molecules involved are still the subject of debate but their role in the disease process makes the study of multigene families in P. falciparum surface proteins a priority. Furthermore, investigations have highlighted the lack of knowledge concerning both pathology and virulence of $P$. falciparum and suggest a role for several other proteins. It is possible that families of which there is little known may play important roles in the disease process.

Little is known about how gene expression is controlled, and the positioning of the genes from different multigene families next to each other make it possible that their transcription is linked. It has yet to be determined whether stevor, rif and var expression are somehow related to each other and whether their sub-telomeric co-localisation is functionally important. Consequently there is a need to understand transcriptional control within multigene families.

This project will study the stevor multigene family of $P$. falciparum. The gene structure and proteins predictions of stevor are very similar to rif and the RIFINs (Figure 1.4) (Cheng et al., 1998). There is also some sequence similarity with the region of highest homology having around $72 \%$ sequence similarity (Kyes et al., 2001). RIFINs are membrane bound RBC surface proteins and it is possible that the products of this gene family called STEVOR are also located on the surface or associated with the RBC membrane (Kyes et al., 1999). Thus, STEVOR could potentially have roles in immune evasion and pathogenesis. These reasons as well as the chromosomal organisation of stevor with var and rif has led to further investigations of the stevor multigene family. 
Initial characterisation of the stevor family (Cheng et al., 1998; Sutherland, 2001) demonstrated that stevor is transcribed in both asexual and sexual stages of $P$. falciparum. However the cellular location of STEVOR has not yet been elucidated. The difference in transcriptional control of multigene families (cf. var and py235) has been established and it will be important to study what mechanisms are employed in stevor transcription. In addition, the transcriptional relationships between the var, stevor and rif gene families are of interest due to their close proximity at each telomere and their possible combined role in antigenic variation, immune evasion and function. In summary, this project aims to:

- Perform a detailed analysis of transcription of the stevor multigene family.

- Characterise STEVOR expression in pRBC

- Localise STEVOR in pRBC 


\section{CHAPTER TWO}

\section{Materials and Methods}

\subsection{Materials, media, solutions and buffers}

All chemicals and reagents used were of analytical grade and were obtained from BDH Chemicals Ltd., Poole, UK, unless otherwise stated. Compositions of media, solutions and buffers are given in Appendix I.

\subsection{Bioinformatics}

Alignment and editing of nucleotide and protein sequences was carried out using the DNASTAR EditSeq ${ }^{\circledR}$ Megalign ${ }^{\circledR}$, ClustalX ${ }^{\circledR}$ and Bioedit ${ }^{\circledR}$ programmes. Sequences were checked against the NCBI Genbank Database (http://www.ncbi.nlm.nih.gov/) using a BLAST® (Basic Local Alignment Search Tool) search. This database was also used to obtain sequences by their accession numbers.

Sequence data for $P$. falciparum stevor sequences were obtained from a BLAST search using the site http://plasmodb.org/PlasmoDB.shtml which contains the finished and draft sequences for $P$. falciparum (3D7) generated at the Sanger Centre (UK), Stanford University (USA), and TIGR/NMRC (USA). This site was also used to assign chromosome locations of the stevor sequences.

\subsection{Cell culture}

\subsubsection{Plasmodium falciparum}

The P. falciparum 3D7 clone (derived from the NF54 isolate (Amsterdam airport)) was used in all experiments and is also the human malaria clone sequenced by the malaria genome project. Parasites were cultured in human $\mathrm{RBC}$ in parasite culture 
medium (Appendix I) (Trager and Jensen, 1977). The pRBC were incubated at $37^{\circ} \mathrm{C}$ in a low oxygen atmosphere $\left(\mathrm{O}_{2}(5 \% \mathrm{v} / \mathrm{v}), \mathrm{CO}_{2}(7 \% \mathrm{v} / \mathrm{v}), \mathrm{N}_{2}(88 \% \mathrm{v} / \mathrm{v})\right)$. Medium was replaced daily, and cultures were maintained at 5-10\% parasitaemia with a $1-2 \%$ haematocrit. Thin blood films were made of $\mathrm{pRBC}$ on microscope slides and these were fixed in methanol and stained with Giemsa. Parasites were visualised by light microscopy of methanol-fixed, Giemsa-stained (Giemsa's staining solution was diluted 1 in 10 in Giemsa staining buffer (Appendix I)). The blood films were examined at 100X magnification under a light microscope.

P. falciparum 3D7 parasites with a truncated PfEMP-3 gene (Waterkeyn et al., 2000) were kindly donated by Dr A Cowman (WEHI, Melbourne, Australia) and maintained in culture as described above by Mrs M. Grainger.

Gametocyte slides for immunofluorescence assays (see section 2.32) were kindly provided by Mrs M. Grainger (NIMR) and Dr. I Delerieu (NIMR). Gametocytes were cultured and collected as previously described (Day et al., 1998b).

\subsubsection{Chinese hamster ovary cell lines}

Chinese Hamster Ovary (CHO) cells that constitutively express CSA, and stable transformants of CHO cells expressing CD36 and ICAM-1, were kindly donated by Dr. Artur Scherf (Institute Pasteur, Paris, France) and maintained in culture as previously described (Scherf et al., 1998).

\subsubsection{Myeloma cells}

The myeloma cell line Sp2/0-Ag14 was kindly donated by Malcolm Strath (NIMR), and maintained in complete medium (Appendix I). Cultures were maintained at $70 \%$ confluency and visualised in an inverted light microscope at $400 \mathrm{X}$ magnification. 


\subsection{Synchronisation of parasites in culture}

\subsubsection{Sorbitol synchronisation}

To remove trophozoite and schizont stages the parasite culture was treated with sorbitol based on the method by (Lambros and Vanderberg, 1979). The cells were pelleted at $500 \mathrm{xg}$ for $5 \mathrm{~min}$, the supernatant aspirated, and the pellet resuspended in at least 10 volumes of $5 \%(\mathrm{w} / \mathrm{v})$ sorbitol in $\mathrm{ddH}_{2} \mathrm{O}$. The suspension was left for $15 \mathrm{~min}$ at $37^{\circ} \mathrm{C}$, washed once in RPMI 1640 without albumax (Gibco BRL, Life Technologies, Paisley, UK) and then placed back in culture. Sorbitol treatment causes the selective lysis of trophozoite- and schizont-infected $\mathrm{RBC}$, leaving only ring-stage parasites.

\subsubsection{Percoll synchronisation}

The parasite culture was pelleted at $500 \mathrm{xg}$ for $5 \mathrm{~min}$. The parasite pellet $(1 \mathrm{~mL})$ was resuspended in $4 \mathrm{~mL}$ RPMI 1640 without albumax, layered onto $10 \mathrm{~mL}$ of $63 \%$ isotonic Percoll (Amersham Pharmacia Biotech, Uppsala, Sweden) and centrifuged at 560xg for $10 \mathrm{~min}$ at room temperature. Ring and trophozoite stages were isolated in the pellet whereas the schizonts were suspended at the gradient interface. Schizonts were collected and washed three times in RPMI 1640 without albumax and centrifuged at $500 x g$ for $5 \mathrm{~min}$. They were then incubated with $1 \mathrm{~mL}$ fresh $\mathrm{RBC}$ in parasite culture medium for $1 \mathrm{~h}$ at $37^{\circ} \mathrm{C}$ in a low oxygen atmosphere to allow merozoite invasion to take place. Sorbitol synchronisation was then performed (Section 2.4.1) to lyse any remaining schizonts. The resulting pellet contained newly invaded $\mathrm{RBC}$ with ring-stage parasites and was used to start a new culture termed time zero. Schizonts collected after the Percoll gradient were used for protein extractions; to make smears for indirect immunofluorescence assays (IFA) (Section 2.32) and to make RBC and pRBC ghosts (Section 2.6). 


\subsection{Isolation of different stages of parasites}

\subsubsection{Gelatin flotation}

Gelatin flotation (Pasvol et al., 1978) was used to select for trophozoites and schizonts with knob-like structures for use in binding experiments and IFA. A parasite culture was centrifuged at $500 \mathrm{xg}$ for $5 \mathrm{~min}$ at room temperature and the supernatant discarded. The volume of pellet was estimated and $2.4 \mathrm{x}$ volumes of parasite culture medium (at $37^{\circ} \mathrm{C}$ ) was added. One volume of Gelofusine (B.Braun Medical Ltd., Sheffield, UK) at $37^{\circ} \mathrm{C}$, equal to the total volume of cells and medium, was then added to the tube and the contents mixed by gentle inversion. The Gelofusine/parasite mixture was incubated for $30 \mathrm{~min}$ at $37^{\circ} \mathrm{C}$. At this stage, two phases are visible; the top brownish layer contained schizonts and trophozoites and the lower layer contained uninfected red cells and erythrocytes with immature forms of the parasite. The top layer was collected and washed three times in binding buffer (RPMI without $\mathrm{H}_{2} \mathrm{CO}_{3}$; pH6.8) for binding experiments (Section 2.8) or used to make smears for IFA (Section 2.32).

\subsubsection{Percoll-sorbitol gradient}

A Percoll-sorbitol gradient was performed as previously described (Kutner et al., 1985) to isolate trophozoites for micromanipulation. The technique is based on the parasite's induction of increased permeability to sorbitol induced in the host cell membrane by growth of the parasite. The gradient was performed on cultures that had been synchronised by Percoll isolation of schizonts, followed by sorbitol treatment. This method is different to the method in Section 2.4.2, as it uses successive gradients of sorbitol and percoll ranging from $90-60 \%$ to separate the different erythrocytic parasite stages. Isotonic percoll (90\%) was made hypertonic by the addition of $6 \%$ (w/v) of sorbitol. This solution was then diluted with phosphate buffered saline (PBS) (Appendix I) containing 6\% (w/v) sorbitol to yield suspensions of $80 \% 70 \%$ and $60 \%$ Percoll. All solutions were heated to $37^{\circ} \mathrm{C}$ before overlaying the gradient (Figure 2.1). A pRBC culture was pelleted at 500xg for $5 \mathrm{~min}$ and resuspended in $2 \mathrm{~mL}$ PBS-sorbitol and overlayed on top of the gradient. Separation of uninfected from infected $\mathrm{pRBC}$ was performed by centrifugation at $1000 \mathrm{xg}$ for $20 \mathrm{~min}$. The trophozoite fraction (Figure 
2.1) was carefully removed for micromanipulation. Smears were also prepared for microscopic examination.

\subsection{Preparation of parasite ghosts for immunofluorescence assay}

This method is based on hypotonic lysis of the RBC as previously described (Dodge, 1963). Uninfected $\mathrm{RBC}$ or $\mathrm{pRBC}$ were incubated at $4^{\circ} \mathrm{C}$ in PBS for $30 \mathrm{~min}$ and then washed three times in PBS at $500 \mathrm{xg}$. The cells were lysed in 50 volumes 10 $\mathrm{mM}$ HEPES (Sigma) and then centrifuged for $40 \mathrm{~min}$ at $20,000 \mathrm{xg}$ and then washed in a further 50 volumes $10 \mathrm{mM}$ HEPES (Sigma) and centrifuged at 20,000xg for $40 \mathrm{~min}$. This left a white layer of ghosts (erythrocyte membranes from both uninfected and infected RBC) on top of a haemozoin-rich layer containing the contents of the infected or uninfected erythrocytes. The ghosts were collected and used to make IFA smears.

\subsection{Micromanipulation of single parasites}

Parasite cultures were synchronised and trophozoites isolated on a Percoll/sorbitol gradient (Section 2.5.2). The pRBC were collected and then centrifuged (500xg for 5 min at room temperature). The pellet was washed twice $(500 \mathrm{xg}$ for $10 \mathrm{~min}$ at room temperature) in Krebs buffered saline (KBS) (Appendix I). Single parasites were selected under phase contrast optics and micromanipulated using micropipettes pulled from GC150-F borosilicate capillary tubes (Clark Electromedical Instruments) (Preiser et al., 1999).

\subsection{Selection of CD36-, ICAM-1- and CSA-binding parasites in vitro}

Panning of parasites on $\mathrm{CHO}$ cells was performed using 10-mL cultures of $70 \%$ confluent $\mathrm{CHO}$ cells. A $1 \%$ haematocrit of parasites prepared using gelatin flotation (Section 2.5.1) were added to $10 \mathrm{~mL} \mathrm{CHO}$ cells. Parasites were harvested and washed in binding buffer (Section 2.5.1) and then resuspended at $1 \%$ haematocrit. This was added to the $\mathrm{CHO}$ cells and incubated for $37^{\circ} \mathrm{C}$ for $30 \mathrm{~min}$, resuspending parasites every 


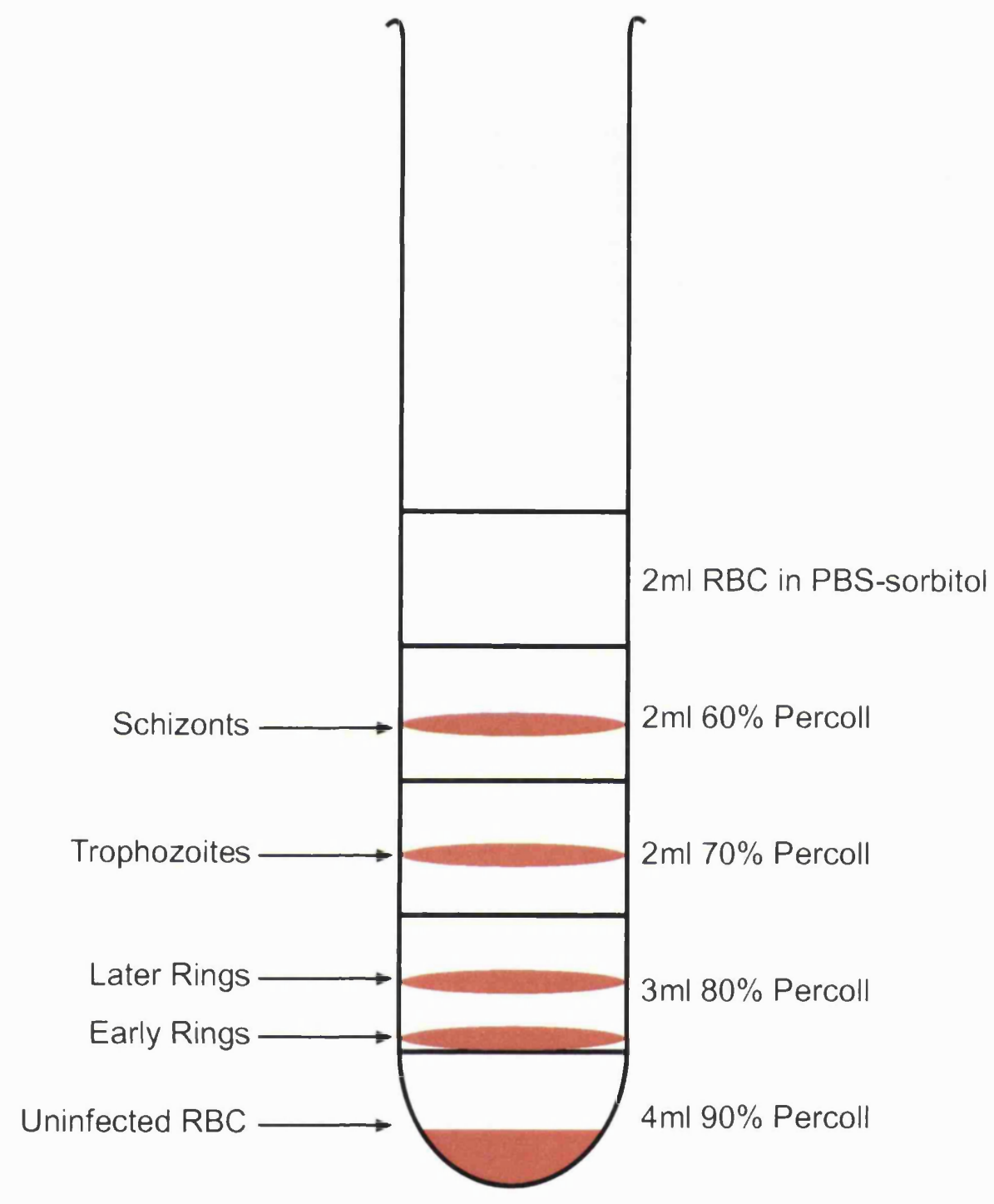

Figure 2.1 Schematic of a typical fractionation of different stages of $P$. falciparum blood stages in a Percoll-sorbitol gradient. The Percoll concentrations and the distribution of parasite stages as assessed by microscopic examination are indicated. $P$. falciparum synchronised cultures were suspended in PBS-sorbitol and layered on the top of a Percoll-sorbitol discontinuous gradient and used to separate trophozoites from uninfected RBC for micromanipulation. 
10 min. Bound parasites were detached using an automatic pipette aid. The released pRBC were centrifuged at $500 \times \mathrm{xg}$ for $5 \mathrm{~min}$ and washed three times with binding buffer and used to prepare total RNA (Section 2.10).

\subsection{DNA preparation}

\subsubsection{High molecular weight DNA}

Parasite cultures $(100 \mathrm{~mL})$ at $10 \%$ parasitaemia and $1 \%$ haematocrit were centrifuged at $500 \mathrm{xg}$ for $5 \mathrm{~min}$ at room temperature. Genomic DNA was extracted as previously described using SDS and Proteinase K (Boehringer Mannheim, Lewes, UK) followed by phenol/chloroform (Sigma, Poole, UK) extraction (Owen et al., 1999). Genomic DNA was resuspended in $1 \mathrm{~mL} 1 \mathrm{X}$ Tris- di-sodium ethylenediaminetetraacetic acid (EDTA) (TE) buffer (Appendix I).

\subsubsection{Intact chromosomal DNA plugs}

For the analysis of intact chromosomal DNA by pulse-field gel electrophoresis (PFGE), intact parasite chromosomal DNA was resuspended into agarose as previously described (Borre et al., 1995). Briefly, pRBC were resuspended in 2\% low melting point agarose (Seaplaque GTG, FMC Rockland, ME, USA) in 1X PBS and dispensed into $1 \mathrm{~mL}$ plug moulds. After the plugs had solidified, they were incubated overnight at $37^{\circ} \mathrm{C}$ in parasite lysis buffer (Appendix I). The plugs were washed several times in $\mathrm{dH}_{2} \mathrm{O}$ and stored in $0.5 \mathrm{M}$ EDTA solution $\mathrm{pH} 7.5$ at $4^{\circ} \mathrm{C}$.

\subsection{Isolation of total RNA from parasite culture}

RNA was isolated from pRBC using the Trizol method essentially as described previously (Kyes et al., 2000). Pelleted $\mathrm{pRBC}$ at approximately 5-10\% parasitaemia were resuspended in 10 pellet volumes Trizol (Gibco BRL, Life Technologies) and incubated at room temperature for $5 \mathrm{~min}$, or stored at $-70^{\circ} \mathrm{C}$ until processed. RNA was extracted with 2 pellet volumes chloroform (Sigma) at room temperature for $3 \mathrm{~min}$, then 
precipitated from the aqueous phase by 0.5 pellet volumes propan-2-ol for $1 \mathrm{~h}$ on ice. RNA was resuspended in 0.5 pellet volumes formamide (Sigma).

\subsection{Nucleic acid quantification}

\subsubsection{DNA}

DNA concentration in solution was estimated by comparing the intensity of band fluorescence for DNA samples electrophoresed on an ethidium bromide (EtBr)(Sigma)-stained agarose gel with bands of known concentration from the $\lambda$ HindIII molecular mass marker (Roche)(Table 2.1).

\begin{tabular}{l|l}
\hline Marker band $[\mathbf{k b}]$ & ng in each band after loading $10 \mu \mathbf{L}$ \\
\hline 0.125 & 1.3 \\
0.564 & 5.2 \\
2.027 & 40 \\
2.322 & 24 \\
4.361 & 45 \\
6.557 & 68 \\
9.416 & 98 \\
23.130 & 240 \\
\hline
\end{tabular}

\section{Table 2.1: Lambda HindIII Marker}

Relative amounts of each band in the $\lambda$ HindIII marker. $\lambda$ HindIII marker was prepared in 1X loading Buffer (Appendix I) to a final concentration of $500 \mathrm{ng} / 10 \mu \mathrm{L}$. This standard was used to quantify DNA on gels. 


\subsubsection{RNA}

RNA concentrations in solution were determined by measuring the absorbance at $260 \mathrm{~nm}$ using a Unicam UV1 spectrophotomer $\left(\mathrm{A}_{260}=1.00\right.$ corresponds to an RNA concentration of $40 \mu \mathrm{g} / \mathrm{mL}$ )

\subsection{Agarose gel electrophoresis}

Fragments of DNA and RNA were separated by electrophoresis in Multi Purpose agarose (Boehringer Mannheim) $(1 \% \mathrm{w} / \mathrm{v}$ in $1 \mathrm{X}$ Tris Borate EDTA (TBE) buffer (Appendix I)) gels containing $0.5 \mu \mathrm{g} / \mathrm{mL}$ of ethidium bromide (EtBr). Gels used to separate RNA also contained 0.02 M guanidinium thiocyanate (Kyes et al., 2000). Fragments of DNA less than $1 \mathrm{~kb}$ in size were separated by electrophoresis in Metaphor agarose (Flowgen, Rockland, ME, USA) (3\% w/v in $1 \mathrm{X}$ TBE buffer) gels containing $\operatorname{EtBr}(0.5 \mu \mathrm{g} / \mathrm{mL})$.

\subsection{Pulse-Field Gel Electrophoresis}

The resolution of whole $P$. falciparum chromosomes was performed on the CHEF DRII (BioRad, Gottingen, The Netherlands) gel electrophoresis machine. The parasite plugs were inserted into a $0.7 \%$ agarose gel (Boehringer Mannheim) in 0.25X TBE buffer. Pulse times and conditions for the gel run were: $90-300 \mathrm{~s}$ ramp at $95 \mathrm{~V}$ for $36 \mathrm{~h}$ followed by $300-720 \mathrm{~s}$ ramp at $85 \mathrm{~V}$ for $36 \mathrm{~h}$ with a $3 \mathrm{~s}$ pause between pulses.

\subsection{Primer design}

External primers were designed around a conserved region of the gene based on six full-length stevor sequences (Accession no. AF065198 to AF065201 from the NCBI Genbank Database(http://www.ncbi.nlm.nih.gov/). Internal primer sequences designed around the polymorphic region of stevor were obtained from Cheng et al., 1998. All primers were synthesised by Oswel (Southampton, UK). 


\begin{tabular}{|l|l|}
\hline$\frac{\text { Internal }}{\text { mRepF1 (sense) }}$ & $\begin{array}{l}\text { AGA TGA ATT CGT GGT ATA T(A/G)T T(T/C)T T(T/C)T } \\
\text { C }\end{array}$ \\
mRepF2 (sense) & AGA TGA ATT CGG GGT TTA GTT GTG TGT GC \\
mRepR (anti-sense) & $\begin{array}{l}\text { TTT AGG ATC CAG AAC CAA A(A/G)(T/C) TGC AAT } \\
\text { ACC }\end{array}$ \\
\hline External & GA(C/T) (C/G)CA GAA CTC AA(A/G) GAA AT(A/T) ATT \\
Smr1 (sense) & $\begin{array}{l}\text { G (anti-sense) } \\
\text { GCA G(A/C)A CCA AAG (C/T)(T/A)G (C/T)AA TAC C }\end{array}$ \\
\hline
\end{tabular}

Table 2.2 Stevor -specific primers

\subsection{Amplification of DNA by Polymerase Chain Reaction (PCR)}

PCR reactions were carried out in a Biometra Uno Thermoblock (Biometra Ltd., Maidstone, UK) or Eppendorf Mastercyler Gradient (Eppendorf, Hamburg, FRG). All PCR reactions were carried out in $500 \mu \mathrm{L}$ tubes and overlaid with $50 \mu \mathrm{L}$ mineral oil (Sigma) to prevent evaporation.

\subsubsection{PCR using external primers}

PCR using external primers was carried out in a volume of $50 \mu \mathrm{L}$ containing approximately $100 \mathrm{ng}$ of template DNA, $10 \mathrm{pM}$ of each primer, $5 \mu \mathrm{L} 10 \mathrm{X}$ PCR Buffer II (100 mM Tris-HCl, $500 \mathrm{mM} \mathrm{KCl)(Roche),} 2 \mathrm{mM} \mathrm{MgCl}_{2}$ (Perkin Elmer, La Jolla, USA), $1 \mathrm{mM}$ each of dATP, dCTP, dGTP and dTTP (Amersham Pharmacia Biotech), and $5 \mathrm{U}$ AmpliTaq polymerase (Roche). The PCR program was as follows: 1 cycle at $94{ }^{\circ} \mathrm{C}$ for $3 \mathrm{~min}, 35$ cycles of $94^{\circ} \mathrm{C}$ for $1 \mathrm{~min}, 62.5^{\circ} \mathrm{C}$ for $1 \mathrm{~min}$ and $72{ }^{\circ} \mathrm{C}$ for $1 \mathrm{~min}$ $30 \mathrm{~s}, 1 \mathrm{cycle}$ at $72^{\circ} \mathrm{C}$ for $10 \mathrm{~min}$ and pausing at $10^{\circ} \mathrm{C} .^{\circ}$

\subsubsection{PCR using internal primers}

A nested PCR using internal primers were carried out in a volume of $25 \mu \mathrm{L}$ containing $2 \mu \mathrm{L}$ of the product of the external primer PCR, $5 \mathrm{pM}$ each mRepF1 and mRepF2 and $10 \mathrm{pM}$ of mRepR, $2.5 \mu \mathrm{L}$ 10X PCR Buffer, $3 \mathrm{mM} \mathrm{MgCl}_{2}, 1 \mathrm{mM}$ each of dATP and 2.5 units of AmpliTaq polymerase. The PCR program was as follows: 1 
cycle at $94{ }^{\circ} \mathrm{C}$ for $3 \mathrm{~min}, 40$ cycles of $93^{\circ} \mathrm{C}$ for $30 \mathrm{~s}, 45^{\circ} \mathrm{C}$ for $50 \mathrm{~s}, 70^{\circ} \mathrm{C}$ for $30 \mathrm{~s}, 1$ cycle at $72^{\circ} \mathrm{C}$ for $10 \mathrm{~min}$ and pausing at $4{ }^{\circ} \mathrm{C}$.

\subsection{Reverse Transcriptase (RT)-PCR}

\subsubsection{DNase treatment}

In order to remove any contaminating DNA, RNA was first treated with DNase. This was carried out in a total volume of $20 \mu \mathrm{L}$. One $\mu \mathrm{L}$ of RNA in formamide was added to $2 \mu \mathrm{L} 5 \mathrm{X}$ RT Buffer (250 mM Tris-HCl, $375 \mathrm{mM} \mathrm{KCl}, 15 \mathrm{mM} \mathrm{MgCl})(\mathrm{Life}$ Technologies), $2 \mu \mathrm{L}$ of $25 \mathrm{mM} \mathrm{MgCl}_{2}, 2 \mu \mathrm{L} 0.1 \mathrm{M}$ DTT (Invitrogen, Gottingen, FRG), $10 \mathrm{U}$ DNase (Boehringer Mannheim) and incubated for $2 \mathrm{~h}$ at $37^{\circ} \mathrm{C}$ before heat inactivating at $70^{\circ} \mathrm{C}$ for $10 \mathrm{~min}$.

\subsection{2 cDNA synthesis}

After DNase treatment, $20 \mu \mathrm{L}$ of the RNA sample was incubated with $1 \mu \mathrm{L}$ $(100 \mathrm{ng} / \mu \mathrm{L})$ random hexamers (Gibco, Life Technologies) at $70^{\circ} \mathrm{C}$ for $10 \mathrm{~min}$. dNTPs at a final concentration of $10 \mathrm{mM}$ and $1 \mu \mathrm{L}$ SuperScript ${ }^{T M}$ II RNase $\mathrm{H}^{-}$RT (Life Technologies) were then added and the sample was incubated at $25^{\circ} \mathrm{C}$ for $10 \mathrm{~min}, 42^{\circ} \mathrm{C}$ for $30 \mathrm{~min}$ and $95^{\circ} \mathrm{C}$ for $3 \mathrm{~min}$. A control tube without RT was included for each reaction and processed in parallel to ensure there was no contamination of the RNA preparation with the genomic DNA.

Amplification of the cDNA using first external primers and then internal primers was carried out as described in Sections 2.15.1 and 2.15.2.

\subsection{Single cell RT-PCR}

Single cell RT-PCR was performed as previously described (Preiser et al, 1999). PCR with external primers was performed by adding $30 \mu \mathrm{L}$ of PCR buffer (containing $0.5 \mu \mathrm{M}$ Smr1 and $1 \mu \mathrm{M}$ of Smf1, $3 \mu \mathrm{L}$ 10X PCR Buffer II, $2 \mathrm{mM} \mathrm{MgCl} 2$ and $1 \mathrm{mM}$ each of dATP, dCTP, dGTP and dTTP, and $2.5 \mathrm{U}$ AmpliTaq polymerase) to 
each tube. Amplification was carried out using the PCR program as described in section 2.15.1. The nested PCR reaction was carried out as described in Section 2.15.2.

\subsection{Cleaning of PCR and RT-PCR products}

PCR and RT-PCR products were cleaned using the QIAquick PCR purification kit (Qiagen, Hilden, The Netherlands) according to the manufacturer's protocol. PCR products were eluted into a final volume of $30 \mu \mathrm{L}$ elution buffer (Qiagen).

\subsection{Cloning into plasmid vectors}

\subsubsection{Ligation reaction}

Cleaned PCR products were cloned into the $\mathrm{pCR}^{\circledR} 2.1$ vector using the Original TA Cloning ${ }^{\circledR}$ Kit (Invitrogen) according to the manufacturer's protocol. These vectors contain single 3' dT-overhangs at their insertion sites. This enables ligation of a PCR product without modifications, as the Taq-based thermostable polymerases used in PCR tends to add single dA to the 3' ends of the PCR product in a non-template-dependent manner (Clark, 1988). Approximately $10 \mathrm{ng}$ PCR product was used in ligation reactions with $50 \mathrm{ng}$ TA vector.

\subsubsection{Transformation}

Ligation reactions were transformed into One Shot ${ }^{\mathrm{TM}}$ competent cells $(E$. coli $\mathrm{NV} \alpha \mathrm{F}^{\prime}$ ) (Invitrogen) provided in the Original TA Cloning® Kit according to instructions supplied by the manufacturer. Cells were selected on 100-mm LB agar (Appendix I) plates containing ampicillin $(50 \mu \mathrm{g} / \mathrm{mL}$ ) (LB Amp) (Sigma) and X-gal (Melford Laboratories Ltd.) $(800 \mu \mathrm{g}$ in $40 \mu \mathrm{L}$ di-methylformamide spread onto LB Amp plates), by resistance to ampicillin and insertional inactivation of the lac $\mathrm{Z}$ gene. Cells transformed by vector containing insert formed white colonies, whereas those with no insert, and hence functional $\beta$-galactosidase, formed blue colonies. 


\subsection{Isolation of plasmid DNA}

White colonies from LB Amp plates (above) were picked into $3 \mathrm{~mL}$ LB broth (Appendix I) containing $50 \mu \mathrm{g} / \mathrm{mL}$ ampicillin and grown at $37^{\circ} \mathrm{C}$ with agitation. Plasmid DNA was isolated using the SNAP Miniprep ${ }^{\circledR}$ Kit (Invitrogen) according to the manufacturer's protocol. Plasmid DNA was eluted into a final volume of $60 \mu \mathrm{L}$ sterile 1X TE buffer.

\subsection{Automated DNA sequencing}

Sequencing reactions were carried out using the ABI Prism dRhodamine Terminator Cycle Sequencing Ready Reaction kit (Perkin Elmer, Applied Biosystems) according to the manufacturer's instructions. This system is based on di-deoxy chain terminator methodology (Sanger et al., 1977).

Approximately $600 \mathrm{ng}$ of pCR2.1 vector containing the insert of interest was used in each sequencing reaction with either the M13 forward primer or the M13 reverse primer. The pCR2.1 vector contains sequences that hybridise to the M13 primers, thereby flanking the insertion site of the ligated PCR product.

Excess dye terminators were removed by ethanol precipitation according to the manufacturer's protocol, and the DNA pellets were resuspended in $2 \mu \mathrm{L}$ sequencing loading dye $(16.5 \% \mathrm{v} / \mathrm{v}$ Blue Dextran/EDTA (Applied Biosystems) in formamide (Amresco, Solon, USA)). Labelled DNA fragments were separated using the ABI PRISM $^{\text {TM }} 377$ DNA Sequencer (Perkin Elmer) and analysed using the DNASTAR EditSeq® programme.

\subsection{Southern hybridisation}

Following agarose gel electrophoresis, DNA was de-purinated in $0.25 \mathrm{M} \mathrm{HCl}$ for 15 min, rinsed in $\mathrm{dH}_{2} \mathrm{O}$ and then neutralised in $0.4 \mathrm{M} \mathrm{NaOH}$ for 30 min with gentle agitation. The DNA was transferred overnight from the gel to a Hybond $\mathrm{N}+$ membrane (Amersham Pharmacia Biotech) by capillary elution in a buffer containing $0.4 \mathrm{M}$ $\mathrm{NaOH}$. The DNA was fixed onto the membrane by cross-linking in a Stratalinker (Stratagene, La Jolla, USA). Membranes were stored at $-20^{\circ} \mathrm{C}$ until required. 
Non-specific sites on the membrane were blocked by incubation for $1 \mathrm{~h}$ at $65^{\circ} \mathrm{C}$ in $5 \mathrm{~mL}$ of hybridisation solution (see Appendix I) or using PerfectHyb ${ }^{\mathrm{TM}}$ Plus Hybridisation Buffer (Sigma) in a Micro-8 hybridisation oven (Hybaid). Approximately $25 \mathrm{ng}$ of the cleaned, nested PCR or RT-PCR product was used as a template for probe synthesis and probes were radiolabelled with $1.11 \mathrm{MBq}$ of $\left[{ }^{32} \mathrm{P}\right]$-dATP (Amersham Pharmacia Biotech) using the Prime-It ${ }^{\circledR}$ II Random Primer Labeling Kit according to the manufacturer's protocol (Stratagene). Unincorporated nucleotides were separated from the probe by purification on $\mathrm{NICK}^{\mathrm{TM}}$ Columns (Amersham Pharmacia Biotech) according to the manufacturer's instructions. The probe was eluted in $400 \mu \mathrm{L}$ of $1 \mathrm{X}$ TE buffer, denatured by boiling for $5 \mathrm{~min}$ and then placed immediately on ice for $5 \mathrm{~min}$. The probe was then added to the hybridisation solution at $65^{\circ} \mathrm{C}$ and the membrane probed overnight.

Unbound probe was removed from the membrane by washing twice in $250 \mathrm{~mL}$ 2X SSPE, $0.1 \% \mathrm{v} / \mathrm{v}$ SDS at RT for $10 \mathrm{~min}$ followed by one wash in $250 \mathrm{~mL} 1 \mathrm{X}$ SSPE buffer, $0.1 \% \mathrm{v} / \mathrm{v}$ SDS at $65^{\circ} \mathrm{C}$ for $15 \mathrm{~min}$. If required, a second wash in $250 \mathrm{~mL} 0.5 \mathrm{X}$ SSPE, $0.1 \% \mathrm{v} / \mathrm{v}$ SDS at $65^{\circ} \mathrm{C}$ for $10 \mathrm{~min}$ was carried out. The membrane was exposed to Kodak Biomax MR Single Emulsion film (Sigma) at $-70^{\circ} \mathrm{C}$ overnight and developed.

\subsection{Northern blotting}

Northern blotting of RNA was performed as previously described (Kyes et al., 2000). Briefly, RNA from 3D7 parasites (see section 2.11) was loaded onto a $1 \%$ agarose gel (see section 2.7). Samples were heated to $60^{\circ} \mathrm{C}$ for $10 \mathrm{~min}$ and placed on ice for $3 \mathrm{~min}$ before loading. The gel was run at $110 \mathrm{~V}$ for $15 \mathrm{~min}$ and then run at $70 \mathrm{~V}$ for $4 \mathrm{~h}$. The gel was soaked in $7.5 \mathrm{mM} \mathrm{NaOH}$ for $30 \mathrm{~min}$ and then transferred overnight to a Hybond $\mathrm{N}+$ membrane (Amersham) using $7.5 \mathrm{mM} \mathrm{NaOH}$. The gel was probed with radiolabelled products of the nested PCR reaction (using genomic DNA as a template) as described in Section 2.23. 


\subsection{Generation of peptide-specific sera}

\subsubsection{Peptide design}

In order to generate STEVOR-specific antibodies, three peptides were designed around conserved regions of homology between STEVOR sequences (Cheng et al., 1998). A cysteine residue was added to the amino-terminal end of the peptides to enable conjugation to a carrier protein. Peptide 1 (CNPHYHNDPELKEII, aa 57-70), peptide 2 (CEPMSTELEKELELLETY, aa 112-126) and peptide 3 (CIWLYRRRKNSWKHECKKHLC aa 281-300) were designed from conserved regions of STEVOR based on a sequence alignment of full length STEVOR sequences obtained from the http://plasmodb.org/PlasmoDB.shtml site (see Appendix II for sequence alignments and location of peptides).

\subsubsection{Antibody generation}

The peptides were conjugated to Imject® Maleimide Activated Mariculture keyhole limpet haemocyanine (KLH, Pierce, Rockford, USA) according to the manufacturer's instructions in order to increase the immunogenicity. The solution was transferred to Slide-A-Lyzer dialysis cassettes (Pierce) and dialysed twice in 5 L PBS. $\mathrm{BALB} / \mathrm{c}$ mice were immunised intraperitoneally with $50 \mu \mathrm{g}$ of the peptide-KLH conjugate in $100 \mu \mathrm{L}$ PBS in an equal volume of complete Freund's adjuvant (Sigma) and boosted intraperitoneally three times at 14-day intervals with $50 \mu \mathrm{g}$ peptide in PBS but in incomplete Freund's adjuvant (Sigma). Serum was collected 7-10 days after the second and third boosts. Similarly, rabbits were immunised with $200 \mu \mathrm{g}$ of the peptideKLH conjugate subcutaneously with the same regimen as above. Rabbit immunisation was performed by Abbott Murex Biotech Ltd. (Dartford, UK). Serum was aliquoted and stored at $-70^{\circ} \mathrm{C}$ or at $4{ }^{\circ} \mathrm{C}$ with $0.05 \%(\mathrm{v} / \mathrm{v})$ sodium azide.

\subsubsection{Affinity selection of antibodies}

Serum was collected after the final boost with peptide 1 (rabbit immune sera) and affinity selected for antibodies that recognised the peptide 1 on nitrocellulose membrane (Sartorius, Epsom, UK). The STEVOR peptide was conjugated to 
maleimide-activated bovine serum albumin (BSA, Pierce) following the manufacturer's protocol. BSA was used because a carrier was required in order to run the peptide onto a gel. KLH would have been inappropriate for this because the immunised mice will have antibodies against this carrier as well as the peptide. A $10 \%$ acrylamide gel (Section 2.28) was loaded with BSA-conjugated peptide electrophoresed (Section 2.28) and transferred to nitrocellulose membrane as described in section 2.29. The membrane was stained with Ponceau-S stain (Sigma) and the strip containing BSA-conjugated peptide was cut out and blocked (Section 2.30). The strips were washed three times in PBS/ $/ 0.5 \%$ Tween at room temperature with agitation and then incubated for $1 \mathrm{~h}$ at with agitation in a 1:20 dilution of immune and pre-immune sera from Rabbit 6344 (Table $2.3 b$ ) in PBS/Tween. The membrane was then washed eight times for 10 min with agitation, including overnight at room temperature in PBS/Tween and then eluted into 2 $\mathrm{mL}$ of $0.2 \mathrm{M}$ Glycine/ $\mathrm{HCl}, 0.15 \mathrm{M} \mathrm{NaCl}, \mathrm{pH} 2.5$. The eluate was neutralised with $2 \mathrm{M}$ Tris base to $\mathrm{pH} 7.5$ and then added to $2 \mathrm{~mL}$ of PBS/Tween containing $1 \% \mathrm{w} / \mathrm{v}$ BSA and used to probe Western blots as described in Section 2.29.

\subsubsection{Clearing of serum using RBC lysate}

Microcentrifuge tubes containing $1 \mathrm{~mL}$ of leukocyte free blood were lysed using 5-10 volumes of cold hypotonic lysis buffer (See appendix I). Cells were centrifuged for $10 \mathrm{~min}, 15,000 \mathrm{xg}$ at $4^{\circ} \mathrm{C}$ and a vacuum aspirator used to remove the supernatant. Washes were repeated until the haemoglobin was depleted (3-5 washes). Lysates were resuspended in PBS containing lysate:anti-peptide 2 antibody at a ratio of 10:1 followed by incubation for $30 \mathrm{~min}$ on a rotator at $4^{\circ} \mathrm{C}$. Cells were then centrifuged for $15 \mathrm{~min}$ at $15,000 \mathrm{xg}$ and the resulting pellet frozen at $-80^{\circ} \mathrm{C}$. To use, pellets were resuspended in TX100 buffer (Appendix I) with protease inhibitors containing containing $0.1 \mathrm{mg} / \mathrm{mL}$ leupeptin (Sigma), $1 \mathrm{mg} / \mathrm{mL}$ antipain (Sigma) and $20 \mu \mathrm{L}$ phenylmethylsulphonyl fluoride (PMSF, 100mM in Propan-2-ol) (Sigma 0.5mM PMSF) just before use.

\subsection{Generation of monoclonal antibodies}

The production of anti-peptide 1 hybridoma was based on the method as described in Harlow and Lane, (1988). Sera from BALB/c mice were tested by Enzyme-Linked Immunosorbant Assay (ELISA) for reactivity to the peptide (Section 2.32.2) after the 
third boost (Section 2.25.2) and the spleen was isolated from the highest responder mouse. Briefly, the spleen from a boosted mouse was teased into a single cell suspension that was fused with a myeloma cell (SP2/0-Ag14) using polyethylene glycol. Hybridomas were selected in complete medium (Appendix I) containing hypoxanthine-aminopterin-thymidine (HAT) (Sigma) to permit growth of successful fusions only. Culture supernatant from the hybridomas was first screened by ELISA to elucidate antibody-producing cells specific for the peptide. Polyclonal serum from the spleen cell donor was used as the positive control. Normal mouse serum was used as the negative control. The hybridomas that were positive by ELISA were then screened by IFA to make sure that the antibodies also reacted in an IFA. Those wells that had hybridomas that were positive by both ELISA and IFA were expanded in order to reclone. Hybridomas that were positive by both tests were then grown in RPMI comp.medium with supplements and single cell cloning was performed by limiting dilution (the cells were plated at 1000 cells/well, 100 cells/well, 10 cells/well, $1 \mathrm{cell} / \mathrm{well}$ and 0.1 cell/well). Supernatants from growing clones were screened using the anti-peptide1 specific ELISA assay and IFA. Those clones that were positive by ELISA in the 1 cell/well and 0.1 cell/well were tested by IFA. Those cells that were positive were then grown and re-cloned by limiting dilution. mAb producing clones were chosen from the plate that had 0.1 cell/well. Supernatants collected from cell fusions and clones were tested for recognition against peptide 1 by ELISA, IFA and Western blot for final characterisation.

\subsection{Labelling of parasite proteins}

\subsubsection{Metabolic labeling}

Asynchronous parasites were radiolabelled by culturing the parasites in the presence of $\left[{ }^{35} \mathrm{~S}\right]$ methionine and $\left[{ }^{35} \mathrm{~S}\right]$ cysteine Promix (Amersham Pharmacia Biotech) for $4 \mathrm{~h}$. Cultures were grown in methionine- and cysteine-free RPMI medium (Life Technologies) with $0.2 \mathrm{mM}$ L-glutamine before the addition of $100 \mu \mathrm{Ci}$ of Promix per $\mathrm{mL}$. After radiolabelling, for 4 hours in the methionine- and cysteine-free medium the parasites were washed in PBS (three times at $500 \mathrm{xg}$ for $5 \mathrm{~min}$ ) and the pelleted pRBC stored at $-70^{\circ} \mathrm{C}$. 


\subsubsection{Biotinylation}

Biotin was used to label proteins on the surface of uninfected and $\mathrm{pRBC}$ proteins. Biotin labels amine groups and is an alternative to radio-iodination in studies using cell surface molecules (Altin and Pagler, 1995). Schizont-infected RBC and uninfected $\mathrm{RBC}(50 \mu \mathrm{L})$ were washed three times in $15 \mathrm{~mL}$ PBS at $500 \times \mathrm{xg}$ for $5 \mathrm{~min}$ to remove protein in the medium. The cells were then biotinylated with $100 \mu \mathrm{g}$ or $300 \mu \mathrm{g}$ of EZ-Link ${ }^{\mathrm{TM}}$ Sulfo-NHS-LC-Biotin (Pierce) in $\mathrm{ddH}_{2} \mathrm{O}$ for $10 \mathrm{~min}$ at room temperature with agitation. The reaction was quenched using $10 \mathrm{~mL}$ of $20 \mathrm{mM}$ glycine (Sigma) in PBS. The cells were pelleted for $5 \mathrm{~min}$ at $500 \mathrm{xg}$ and then washed three times is PBS as above. Lysates were made as described in section 2.27 and proteins were immunoprecipitated as described in section 2.30. Two positive control antibodies; anti-Band 3 polyclonal rabbit serum (Pinder et al., 1995) and anti-Glycophorin A (GPA) IgG2a monoclonal antibody (BRIC163) (IBGRL Research Products, Elstree UK) were used for immunoprecipitation of biotinylated proteins. Immunoprecipitated proteins were then run on an SDS- Polyacrylamide gel electrophoresis gel (PAGE) as described in Section 2.28 and detected by Western Blot as described in Section 2.29.

\subsection{Preparation of protein extract}

Approximately $100 \mu \mathrm{L}$ of pelleted schizont-infected RBC (Section 2.5.2) were snap frozen on dry ice and stored at $-70^{\circ} \mathrm{C}$. The parasite material was thawed on ice and to approximately $100 \mu \mathrm{L}$ of pellet, $500 \mathrm{~mL}$ of TritonX-100 buffer containing 0.1 $\mathrm{mg} / \mathrm{mL}$ leupeptin, $1 \mathrm{mg} / \mathrm{mL}$ antipain and $20 \mu \mathrm{L}$ PMSF (100mM in Propan-2-ol) were added. The resulting parasite solution was incubated on ice for $40 \mathrm{~min}$ with vortexing every $10 \mathrm{~min}$, and then centrifuged at $10,000 \mathrm{xg}$ at $4^{\circ} \mathrm{C}$ for $30 \mathrm{~min}$ and the supernatant removed.

After the supernatant was removed, $50 \mu \mathrm{L}$ of SDS extraction buffer (Appendix I) was added to the pellet and the cells sonicated to bring them into suspension. The parasite suspension was heated to $100^{\circ} \mathrm{C}$ for $5 \mathrm{~min}$, then a further $450 \mu \mathrm{L}$ of TX-100 extraction buffer with protease inhibitors was added before centrifugation at $10,000 \mathrm{xg}$ 
for $20 \mathrm{~min}$ at $4^{\circ} \mathrm{C}$. About $500 \mu \mathrm{L}$ of SDS-denatured supernatant was extracted. Protein samples were stored at $-70^{\circ} \mathrm{C}$.

\subsection{Protein separation}

SDS-PAGE separating gels of $1 \mathrm{~mm}$ thick and $10 \%$ acrylamide content (Appendix I) with a stacking gel (Appendix I) were used. Ten $\mu \mathrm{L}$ each of the protein samples (Section 2.20) diluted 1:1 in SDS sample buffer (Appendix I) were loaded per lane. Samples were boiled for $5 \mathrm{~min}$ prior to loading and electrophoresis was carried out at $25 \mathrm{~mA}$ in running buffer (Appendix I) for approximately $40 \mathrm{~min}$. NuPAGE 4-12\% Bis-Tris pre-cast gels (Invitrogen) were also used to separate proteins according to the manufacturer's instructions. A Broad Range Prestained Protein Marker (New England Biolabs) was used (Marker sizes: 7.2, 21.9, 29.6, 35.7, 50.7, 83, 123, 203 kilodaltons $(\mathrm{kDa})$ ). Proteins in the gel were stained in Coomassie blue stain (Appendix I) for $20 \mathrm{~min}$, followed by an incubation in de-stain solution (Appendix I) until the separated proteins could be visualised easily.

\subsection{Western blot analysis}

Proteins were electrophoretically transferred from a polyacrylamide gel to Nitrocellulose Extra blotting membrane (Sartorius, Epsom, UK). Transfer was carried out in transfer buffer (Appendix I) for $1 \mathrm{~h}$ at $30 \mathrm{~V}$ using the NuPAGE ${ }^{\mathrm{TM}}$ Electrophoresis System (NOVEX) according to the manufacturer's instructions.

Non-specific binding sites on the membrane were blocked by incubation with PBS/Tween $20(0.1 \% \mathrm{v} / \mathrm{v})$ (Sigma) containing skimmed milk powder $(5 \% \mathrm{w} / \mathrm{v})(\mathrm{Marvel}$, Wirral, UK) overnight at $4^{\circ} \mathrm{C}$, with agitation. All following incubations and washes were carried out at room temperature with agitation. The membrane was washed three times for $10 \mathrm{~min}$ in $\mathrm{PBS} / \mathrm{Tw}$ ween prior to incubation for $1 \mathrm{~h}$ in polyclonal mouse or rabbit serum diluted 1:2000 in PBS/Tween with $1 \%(\mathrm{v} / \mathrm{v})$ BSA or affinity-purified serum (see Section 2.24.3). Unbound antibody was removed by washing the membrane three times for $10 \mathrm{~min}$ in $\mathrm{PBS} / \mathrm{Tw}$ ween. Bound antibody was detected by incubation with horseradish peroxidase (HRP)-conjugated goat anti-rabbit IgG (BioRAD) diluted 
1:4000 or HRP-conjugated goat anti-mouse IgG diluted in 1:2000 for $1 \mathrm{~h}$, followed by washing six times for $10 \mathrm{~min}$ in PBS/Tween.

Modifications were made for the detection of biotinylated proteins. Membranes were incubated in PBS/Tween $20(0.1 \% \mathrm{v} / \mathrm{v})$ with $1 \%$ BSA because Marvel skimmed milk powder contains small traces of biotin. Biotinylated proteins were detected by HRP-conjugated streptavidin (Pierce, Rockford, Il, USA) diluted 1:15,000 in PBS/Tween.

The presence of peroxidase was detected by enhanced chemiluminesence using the SuperSignal ${ }^{\circledR}$ West Pico Chemiluminesence Substrate (Pierce) according to the manufacturer's instructions. The membrane was exposed to Kodak Biomax MR Single Emulsion film (Sigma) for 1-30 s and the film developed.

\subsection{Immunoprecipitation}

Schizonts were labelled with $\left[{ }^{35} \mathrm{~S}\right]$ methionine and $\left[{ }^{35} \mathrm{~S}\right]$ cysteine Promix (Section 2.26.1), snap frozen and stored at $-70^{\circ} \mathrm{C}$ until required when they were thawed on ice and TritonX-100 and SDS extractions were performed (as described in Section 2.27). To $100 \mu \mathrm{L}$ of TritonX-100 extract and SDS extract, $3 \mu \mathrm{L}$ of pre-immune sera was added, vortexed and incubated overnight on ice. Each sample was transferred to a fresh tube containing $50 \mu \mathrm{L}$ dry Protein G Sepharose beads (Amersham Pharmacia) (pre-treated according to the manufacturer's instructions), vortexed and then placed on a rotator and mixed for $60 \mathrm{~min}$ at $4^{\circ} \mathrm{C}$. The beads were pelleted by centrifugation and the supernatant removed. To the supernatant, $3 \mu \mathrm{L}$ of immune or pre-immune sera was added and incubated overnight. The samples were then immunoprecipitated with Protein $G$ and then washed twice in $1 \mathrm{~mL}$ of immunoprecipitation wash buffer 1 (Appendix I) and four times in immunoprecipitation wash buffer 2 (Appendix I). The antigen-antibody complexes were then solubilised by adding SDS PAGE sample buffer (1X) and boiling for $5 \mathrm{~min}$. This mixture was then centrifuged and the supernatant collected.

To visualise labelled proteins, the supernatant was loaded $(20 \mu \mathrm{L})$ onto a precast 4-12\% Bis/Tris Novex gel and separated (Section 2.28). The gel was fixed in de-stain solution for $20 \mathrm{~min}$ before washing three times for $5 \mathrm{~min}$ in $\mathrm{dH}_{2} \mathrm{O}$ and soaking for 20 min in $7 \mathrm{~mL}$ of scintillant (Amplify; Amersham). The gel was put on a dryer for $1 \mathrm{~h}$ and exposed to Kodak Biomax MR Single Emulsion film. 


\subsection{Enzyme-Linked Immunosorbant Assay (ELISA)}

STEVOR peptide (50 $\mu \mathrm{L}$ at $4 \mu \mathrm{g} / \mathrm{mL}$ diluted in PBS was added to the wells of a 96-well, flat-bottom Polysorb ELISA plate (NUNC, Naperville, IL, USA) and the coated plate incubated overnight at $4^{\circ} \mathrm{C}$ in $100 \%$ humidity. The antigen was removed by inversion of the plates and nonspecific binding sites were blocked by adding to each well $200 \mu \mathrm{L}$ of PBS containing $5 \% \mathrm{w} / \mathrm{v}$ skimmed milk powder and incubating the plate at $37^{\circ} \mathrm{C}$ for $1 \mathrm{~h}$, followed by three washes in ELISA washing buffer (Appendix I). Twelve two-fold serial dilutions (starting at 1:10) were prepared using $50 \mu \mathrm{L}$ each of rabbit or mouse immune and pre-immune serum (as a negative control) in diluent buffer (PBS containing 1\% BSA) and these were added to assigned wells. After $1 \mathrm{~h}$ at $37^{\circ} \mathrm{C}$, the serum samples were discarded and the plates washed three times as above. To detect bound STEVOR peptide-specific antibodies, $50 \mu \mathrm{L}$ of goat anti-rabbit alkaline phosphatase-conjugated IgG (1:10,000 dilution in diluent buffer; Appendix I) or goat anti-mouse alkaline phosphatase-conjugated IgG (1:2000 dilution in diluent buffer) (Southern Biotechnology, Birmingham, USA) was added for $1 \mathrm{~h}$ at $37^{\circ} \mathrm{C}$. Unbound IgG was removed by washing four times as above. To reveal the amount of specific antibody bound, $1 \mathrm{mg} / \mathrm{mL} p$-nitrophenylphosphate-sodium (PNPP) salt (Sigma) in diethanolamine buffer (Appendix I) was added to the wells and the reaction left to develop at room temperature. Plates were read using a MRX microplate reader (Dynex Technologies, Billinghurst, UK) at Abs405.

\subsection{Indirect immunofluorescence assay (IFA)}

Thin blood smears were made from $P$. falciparum 3D7 pRBC. They were airdried, wrapped in foil and stored with silica gel (Sigma) at $-20^{\circ} \mathrm{C}$ until required when they were dried at $37^{\circ} \mathrm{C}$ for $5 \mathrm{~min}$, fixed in acetone for $2 \mathrm{~min}$ and then air dried. Wells were marked onto the smear with an indelible marker. All washes were for $5 \mathrm{~min}$ in PBS with agitation and antibodies and sera were diluted in PBS containing $1 \% \mathrm{w} / \mathrm{v}$ BSA. Details of the primary and secondary antibodies used are given in Tables $2.3 \&$ 2.4. The primary antibody $(10 \mu \mathrm{L})$ was added to the smear and incubated in a humid 
chamber for $30 \mathrm{~min}$. The antibody was rinsed off with PBS and the slide washed twice and then dried with a hairdryer. The appropriate second antibody $(10 \mu \mathrm{L})$ was added to each well and the slide incubated for $30 \mathrm{~min}$ as above. Slides were washed as before, briefly placed in 4,6-diamidino-2-phenylindole (DAPI, $0.5 \mu \mathrm{g} / \mathrm{mL}$ in PBS) (Sigma) which intercalates with DNA and washed once more, briefly placed in Evans blue solution $(100 \mu \mathrm{g} / \mathrm{mL}$ in PBS) to stain the $\mathrm{RBC}$ membrane and washed again and then dried. A drop of Citifluor AF1 (Citifluor Ltd., London, UK) was added to each well and the wells covered with a coverslip and sealed with nail varnish. When the smears were double stained, each antibody was added in a separate step. The slides were stored in the dark at $4^{\circ} \mathrm{C}$ and viewed on a fluorescence microscope (Zeiss, West Germany) under $63 \mathrm{X}$ magnification or a Dual Imagiser (Leica microscopy, FRG) under 100X magnification.

\begin{tabular}{|c|c|c|c|}
\hline Antibody & Specificity & $\begin{array}{l}\text { Working } \\
\text { dilution }\end{array}$ & Source \\
\hline $\begin{array}{l}2 \mathrm{~F} 10 \text { (mouse monoclonal } \\
\text { antibody IgG) }\end{array}$ & MSP-1 & $1: 50$ & $\begin{array}{l}\text { Mr. T. Scott-Finnigan, } \\
\text { NIMR, UK (Blackman et } \\
\text { al., 1994) }\end{array}$ \\
\hline $\begin{array}{l}61.2 \text { (mouse monoclonal } \\
\text { antibody IgG) }\end{array}$ & $\begin{array}{l}52 \quad \mathrm{kDa} \\
\text { rhoptry protein }\end{array}$ & $1: 100$ & $\begin{array}{l}\text { Dr. AA Holder, NIMR, UK } \\
\text { Unpublished. }\end{array}$ \\
\hline $\begin{array}{l}\text { B28 (mouse polyclonal } \\
\text { antibody IgG) }\end{array}$ & PfSB-1 & $1: 100$ & $\begin{array}{l}\text { Dr. C. Braun-Breton, } \\
\text { (Blisnick et al., 2000) }\end{array}$ \\
\hline $\begin{array}{l}\text { D662 (mouse polyclonal } \\
\text { antibody) }\end{array}$ & PfEMP-3 & $1: 100$ & $\begin{array}{l}\text { Dr. A Gruner, Institute } \\
\text { Pasteur, France (Gruner et } \\
\text { al., 2001) }\end{array}$ \\
\hline
\end{tabular}

Table 2.2a Primary Antibodies used in IFA

\begin{tabular}{l|l}
\hline Antibody & Working dilution \\
\hline Anti-peptide 1 pre-immune sera(Rabbit 6344) & $1: 100$ \\
Anti-peptide 1 Immune rabbit sera (Rabbit 6344) & $1: 100$ \\
Mouse normal sera & $1: 20$ \\
Anti-peptide 2 immune mouse sera & neat \\
Anti-peptide 3 immune mouse sera & $1: 20$ \\
\hline
\end{tabular}

Table 2.2b Anti-STEVOR peptide antibodies 


\begin{tabular}{l|ll}
\hline Immunoconjugate & Working dilution & Source \\
\hline $\begin{array}{l}\text { Goat anti-rabbit IgG- FITC } \\
\text { (Stock concentration 4.5mg/mL) }\end{array}$ & $1: 200$ & Sigma \\
$\begin{array}{l}\text { Goat anti-mouse IgG -FITC } \\
\text { (Stock concentration } 1.1 \mathrm{mg} / \mathrm{mL})\end{array}$ & $1: 50$ & Sigma \\
Goat anti-mouse IgG -TR & $1: 100$ & $\begin{array}{l}\text { Dr. M. Blackman, NIMR, UK } \\
\text { (Blackman } \text { et al., 1996) }\end{array}$ \\
Goat anti-rabbit IgG-TRITC & $1: 50$ & Sigma \\
\hline
\end{tabular}

Table 2.3 -Secondary antibodies used in IFA 


\section{CHAPTER THREE}

\section{DNA analysis of the stevor multigene family in the 3D7 clone of P. falciparum}

\subsection{Introduction}

Multigene families in malaria are highly polymorphic with individual families comprising different numbers of genes. These range between over 200 genes for the vir and rif multigene families (del Portillo et al., 2001; Kyes et al., 1999) and between 3540 genes in the Py235 and var gene families (Khan et al, unpublished; Borre et al., 1995; Kyes et al., 2001). The large degree of polymorphism between genes of the same family makes it difficult to study their transcription. For example, a study of the clonal variation of the rif gene family at the transcriptional level was not possible due to the lack of universal primers (Kyes et al., 1999). Similarly, it was with difficulty that universal primers were designed against the highly polymorphic var gene family and these only span a small region (DBL-1) within the large and variant first exon (Taylor et al., 2000a). Thus, degenerate primers need to be carefully designed and characterised to accurately analyse genes within multigene families.

The stevor multigene family consists of approximately 33 copies of a polymorphic gene in the 3D7 clone and this number varies between different parasite lines. The W2 cloned line contains 30 copies while the Dd 2 cloned line contains 34 copies of stevor. By extrapolation of this data, it is thought that there are between $30-40$ copies of stevor per genome (Cheng et al., 1998). Stevor genes are predominantly located in the subtelomeric regions of all the parasite's chromosomes. However, copies of stevor are also found further towards the centromere, up to $500 \mathrm{~kb}$ from the telomere. The chromosomal distribution of stevor for 3D7 is depicted in Figure 3.1. The distribution of stevor is inconsistent between cloned lines as the number of copies varies between chromosomes. For example, on the Dd2 chromosomes 2 and 3, stevor mapped to subtelomeric YACs of only one arm of each chromosome but in 3D7 mapped to both arms of these chromosomes (Cheng et al., 1998). 


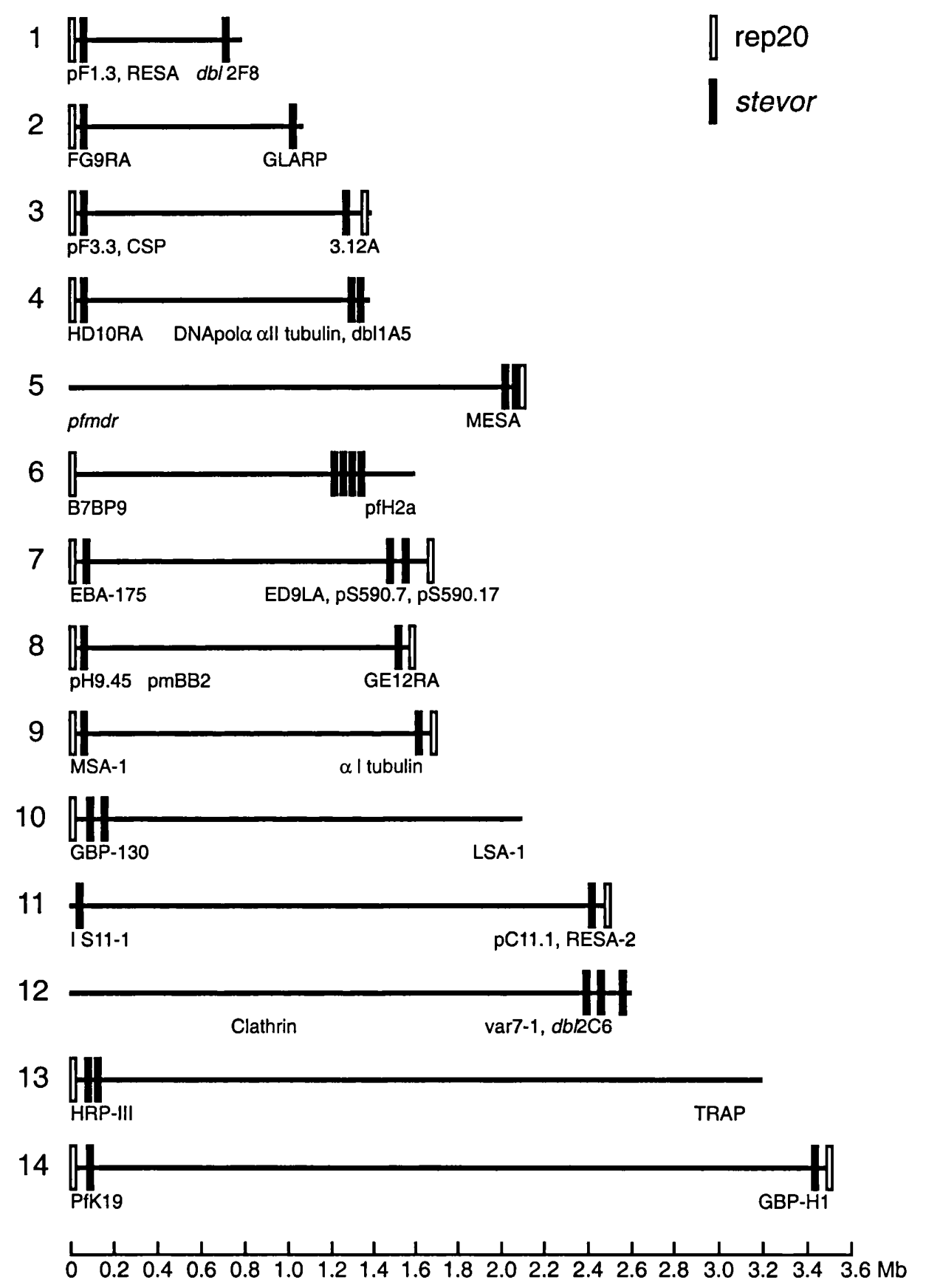

Figure 3.1 Diagram of the organisation of stevor genes on the 14 chromosomes of P. falciparum 3D7 based on the location of stevor containing YACs and the number of copies in each YAC. Modified from Cheng et al., (1998). 
Stevor genes are $\sim 1 \mathrm{~kb}$ in size, with small size polymorphisms. Each gene consists of two exons. The first exon is typically around $65 \mathrm{bp}$, containing a start codon and putative signal peptide. The second exon encodes the remainder of the protein, which includes two putative transmembrane domains flanking the area of highest sequence polymorphism between stevor genes and a highly positively charged Cterminal putative cytoplasmic domain (Figure 3.2). Initial characterisation of this multigene family has shown that stevor is transcribed during the erythrocytic stages of the parasite life cycle in several P. falciparum clones (Cheng et al., 1998). However, detailed transcription studies over the course of the parasitic life cycle have not been explored.

The aim of the work described in this chapter was to design and characterise degenerate primers which could subsequently be used to perform a detailed study of stevor. All analysis was performed using genomic material from the P. falciparum 3D7 clone. Of all the sequences amplified from the genomic DNA of the W2 and 3D7 line only one sequence amplified was the same between the two clones demonstrating the high amount of sequence diversity. Since the 3D7 genome is being sequenced, results obtained using genomic material from this clone allow easy comparison against database sequences. Two sets of primers based around the region of highest polymorphism were developed as described in section 2.10 (Figure 3.2) to amplify and characterise the stevor repertoire. The external primers span a region of $600 \mathrm{bp}$ while the nested primers span a region of around $300 \mathrm{bp}$ (Cheng et al., 1998). The amplified products were then used to determine the specificity and universality of these primers for stevor genes and the stevor repertoire. 


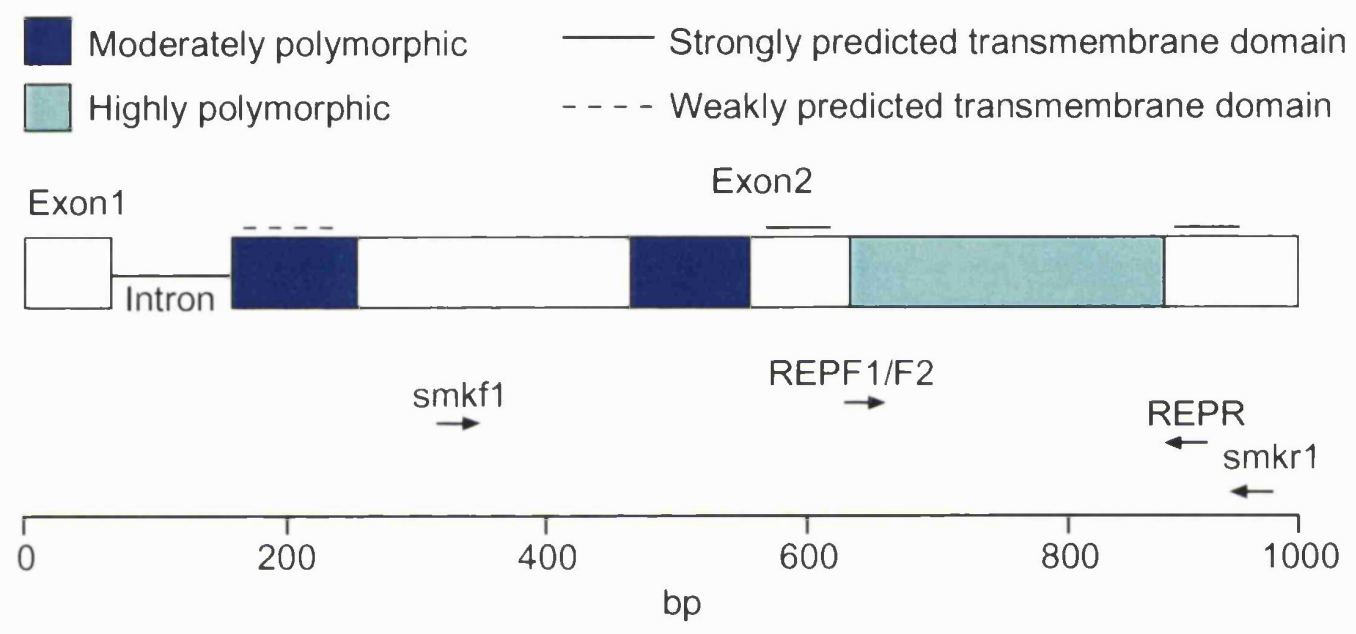

Figure 3.2 Schematic representation of the stevor genes. The external (smkfl and smkf2) and internal (RepF1/F2 \& RepR) primers used in this study are indicated. The stevor gene is $\sim 1000 \mathrm{bp}$ in length. 


\subsection{Results}

\subsubsection{PCR analysis of stevor specific primers}

In order to determine the specificity of the primers, PCR was performed on genomic DNA with both sets of primers (external and nested) (Section 2.10). Multiple products of approximately the expected sizes were detected after electrophoresis when separated on a 3\% Metaphor agarose gel (Figure 3.3A). Two different sized products each of $\sim 600 \mathrm{bp}$ were generated with the external primers and 4 different sized products between 250 and $300 \mathrm{bp}$ were generated with the internal primers. To confirm that the products were derived from stevor, the PCR products from the nested PCR reaction were cloned into PCR 2.1 vector and sequenced. As stevor and rif sequences are very similar (Figure 1.4), the sequences were then compared with available sequences from the NCBI malaria database (Section 2.2), which confirmed that the products were indeed derived from stevor sequences. For a detailed analysis of sequencing results see section 3.2.3.

\subsubsection{Southern blot analysis of 3D7 chromosomes using stevor specific probes}

To determine the universality of the stevor-specific primers, a Southern blot analysis of 3D7 chromosomes resolved by PFGE was performed. PFGE resolved nearly all 14 chromosomes, with both chromosomes 4 and 5, and also 6 and 7 seen to comigrate (Figure 3.3BI). Radiolabelled nested stevor PCR products (Section 3.2.1) hybridised with similar intensity to all 14 chromosomes except chromosomes 9 and 10 (Figure 3.2B). Hybridisation to chromosomes 9 and 10 was with less intensity compared to those of the other chromosomes.

To confirm that the results were not due to cross-hybridisation of the probe to the same/similar sequence on different chromosomes, different cloned PCR products obtained from the nested PCR reaction were used to probe a chromosomal blot. Three clones for which inserts had been sequenced and found to be different (Figure 3.3B) were used as probes and these appeared to hybridise to individual chromosomes $(1,8$ and 4/5) (Figure 3.3B). These results demonstrated that the sequence variability between individual genes was enough to distinguish their different chromosomal 
A

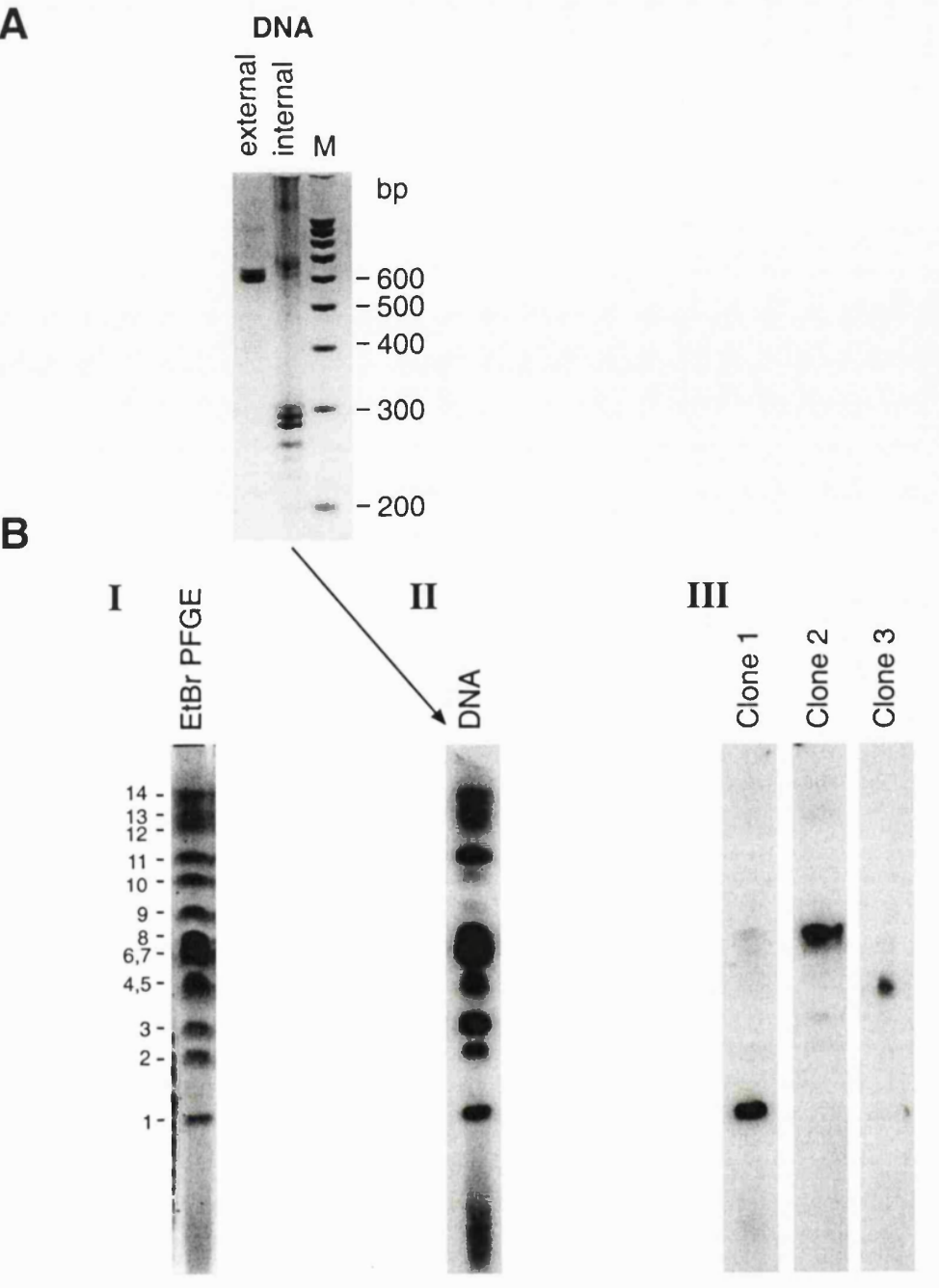

B

Figure 3.3 PCR analysis of stevor. (A) PCR products of 3D7 genomic DNA amplified with external (smkf1 and smkf2) and internal (RepF1/F2 \& RepR) primers.

(B) Products of the nested PCR reaction were then used to probe a Southern blot of P. falciparum 3D7 chromosomes. The chromosomes were resolved by PFGE. DNA from these gels was transferred onto nylon membranes and probed. I) PFGE of 3D7 resolved chromosomes stained with ethidium bromide. II) Probing with radiolabelled nested PCR product obtained from DNA. III) Products from a nested PCR reaction of genomic DNA were cloned and DNA from the individual clones was used to generate radio-labelled probes. 
locations. It was concluded that using the aforementioned primers, a single nested PCR reaction amplified stevor gene products from all chromosomes.

\subsubsection{Repertoire of stevor obtained when using these primer sets}

In order to determine the number of unique stevor genes obtained when using the above primer sets, 53 clones were sequenced. The clones were obtained from three separate nested PCR reactions to ensure that there was no PCR bias. Before any further analysis was performed, each sequence was first compared with the $P$. falciparum NCBI Genbank Database (http://www.ncbi.nlm.nih.gov/) to ensure that the cloned sequences corresponded to stevor. The nucleotide sequences were appropriately translated before alignment, to characterise sequence differences at the protein level. These protein sequences were aligned using ClustalX, which uses the Neighbour Joining (NJ) method (Saitou and Nei, 1987), and a repertoire of predicted stevor primary sequences was obtained. As the clones were only sequenced once, any differences in the degenerate primer regions of the sequence were disregarded and clones were only considered unique if there were at least three residues that were different between sequences. As the sequences were, at most, $300 \mathrm{bp}$ in length, and Taq polymerase makes errors on average at a rate of 1 in 300 , over 3 differences in the translated sequence would make certain that these were genuine differences between sequences (Perkin Elmer Product Information). Hence we were able to identify a total of 21 unique sequences (termed a-u) within the 53 clones sequenced (Figure 3.4). Sequence u (PFB0065w found on chromosome 2) was originally left out of the analysis as a BLAST search assigned it as a rif sequence. However, it has since been demonstrated that existing programs have a high error rate in assigning $3^{\prime}$ intron boundaries (Huestis et al., 2001). A new algorithm to predict $3^{\prime}$ intron splice sites was developed, and application of this programme to the published chromosome 2 and 3 data has now suggested that this gene is a stevor (Huestis et al., 2001).

The majority of sequences were discovered only once (Figure 3.5) confirming further that the primers used are universal for most stevor genes. However, one sequence was found 7 times and two sequences were found 8 times. The frequency (number of times any given sequence is found) for the stevor clones was determined as shown in figure $3.5 \mathrm{~B}$. To determine the probability of obtaining a unique sequence by 


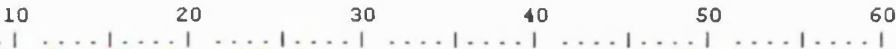
RIRGICFCSL GSVILTYIGT TAAKVACYFY RGCWVELYIR YIYISYASIY VFSSYSIRYS RIRGIYFCSL GSVILTYIGT TAAKGAAIST AGAUVELYIR YIYISYASIY VFSSYSIRYS RIRGICFCSL GSVILTYIGT TAAKGAAIST A-----GAGS KNCIYAISIF HHLNHKSMSS RIRGIYFCSL GSVILTYIGT TAAKGAAIST A-----GAGS KNCIYAISIF HMLNHKSHSS RIRGICSCSV GSAFLTILGF AFAKAAAMKA V-A-DYTAF KTCVSSISTF NLLDSSTLLS RIRGICSCSV GSAFLTILGF AFAKAAAMKA V-A--DYTAF KTCVSSISTF NLLDSSTLLS RIRGIYSCSV ISAFLTHLGL TAAKTAAKGA L-A--EYAAY ETCLSSIPIF SLPGNSTVFS RIRGLVVC--

RIRGIYFCSV STVFLTLIAL AFAKKAAVAS L-V-YYGEAF QNCVSSSSLF YIFDSVSLTT RIRGICFCSI GNALLTMFGL AVAKKASDA I-LPVVNAI KKCVSSSSLF HIFHGGSLTT RIRGICSCSV GSALLTTIGV CATKAALIAK F-AEV--PK N-CIYTISIL DHLTYESHSS RIRGICSCSV GSFLLTMSGL YFAKAAVLKY I-ASVG-PPY NACASTINIY NHIAGGS--RIRGICSCSV GSFLLTHFGL HAAKVAAVAA VLSTYGKT-- STCSSVLSIT NMFNPATLQS RIRGICSCSV GSFLLTMFGL HAAKVAAVAA VLSTYGKT-- STCSSVLSIT NHFNPATLQS RIRGIYSCSV LSAFLTTLGL IFAKKAALAH F--TVHGTOY STCTSYVTIF NHLNGESMIS RIRGICSCSI GSFLLTMFGL HAARAAALAH F--TVHGTQY STCTSYVTIF NLLNGESMIS RIRGICSCSV GSLFLTFIGL AAARATDVAA LNITFNGINY SIYATYVTIL NAFNEASMIA RIRGICSCSI GSTLLTLIGL ALAKKAAVDA LAS--YATSH KICVDSINIF NHIAGIATLRIRGICSCSI GSTLLTLIGL ALAKKAAVDA LAS--YATSH KICVDSINIF NHIAGSA--RIRGLVVCAL SSALVGKYGI AAAASAAKGV L-_...-- ---QAFINAL PSISSSQLTN RIRGICFCSV VUSYQCSVCM LLKLLLLLPS FLPMEKLQLV HOFFLLICLI LLHYSOFFVO

$\pi \pi * \pi+$

\begin{tabular}{|c|c|c|c|c|}
\hline & & & & \\
\hline & & & & \\
\hline LC $-----C$ & LRIRCNFCCN & FSALUYCYFG & AASVLQLWFU & IL- \\
\hline LC $------C$ & LRIRCNFCCN & FSALUYCSFG & SGS--- & \\
\hline TALG $--T$ & VGCAADYAS- & VOLLLVQFFC & LVNL QL U-F W & IL- \\
\hline ALTALG $--T$ & VGCAADYAS- & GATSVSA IFL & PCGIAIL-VL & DPK \\
\hline FQAIS--TC & VEVLLDMPGI & MQLLUMAAFY & PUVLQIL-FL & NL- \\
\hline FQAIS--TC & VEGATDMAGT & IATP AMAAFY & PWGIATL-VL & DPK \\
\hline ALQAGTE-IC & ATHASDLAGI & ISTPAMAAFE & $A L-V L$ & DPK \\
\hline---------- & --- ATDMAGT & IATPAMAAFY & PWGIAVL-VL & DPK \\
\hline A IQTASKCAS & LAGATDAGGT & AAGAAMGIFY & $I L-V L$ & DPK \\
\hline ALKATEACAN & VAGP-DIVIP & ATGAAIGAFP & $T L-V L$ & DPK \\
\hline ALKALSI--- & QACASDLAGT & MGIFY & $A L-V L$ & DPK \\
\hline ----------- & - ACVSDITGT & AASABTAIFE & PCGIAAL-VL & DPK \\
\hline VLCATNG--C & TIGASDVVLP & ATSAAMGTFQ & PCGNCNFGFU & DPK \\
\hline VLCATNG--C & TIGASDVULP & ATS AAMGTFQ & PCGNCNFGFU & DPK \\
\hline AIKVGTGI-C & ATGLSDLATP & AATS ANAMFG & PFGIAAL-VL & DPK \\
\hline A IKVGTGV-C & ATGLSDLATP & TATSANAMFG & PFGIAAL-VL & DPK \\
\hline AIQAGVGA-T & VDGLADMLTP & AAASASAIFG & GFGIATL-VL & DPK \\
\hline$-------n-n$ & -VLLIQYLLQ & LLLLQFLFLV & VLQL WF W-IL & --- \\
\hline--------- & -VCAADLTVP & AATSAATIFV & PCGIAAL-VL & DPK \\
\hline - - - - - - & -- ILNG INF F & YETS I KS AFT & -FGVLQLUFU & IL - \\
\hline MV-- - - --- & VLLVLLMYYL & LQVLL WE HFN & LUVLQL W-FW & IL- \\
\hline
\end{tabular}

Figure 3.4 ClustalX alignment of 21 unique stevor sequences (a-u) obtained from 53 clones after cloning DNA products from nested PCR reactions using stevor-specific primers. Sequences are arranged in descending order of similarity. The green shading shows amino acid identity between the sequences. The * depicts $100 \%$ identity between all 21 sequences. 
Figure 3.5 (A) DNA sequences from cloned stevor PCR products were aligned against sequences deposited in the $P$. falciparum http://plasmodb.org/PlasmoDB.shtml database using BLAST. All the unique sequences found are listed in column 1. The number of times a sequence was found in the analysis and its chromosomal location are listed in columns 2 and 3 respectively. gDNA= genomic DNA $\mathrm{n}=$ number of clones sequenced (B) The probability of finding a particular stevor sequence is depicted by calculating the frequency distribution of stevor sequences amplified by PCR. 
A

\begin{tabular}{c|cc}
\hline Sequence type & gDNA & Chromosome \\
\hline $\mathrm{a}$ & 8 & 3 \\
$\mathrm{~b}$ & 1 & 3 \\
$\mathrm{c}$ & 5 & 14 \\
$\mathrm{~d}$ & 1 & 14 \\
$\mathrm{e}$ & 1 & 10 \\
$\mathrm{f}$ & 1 & 14 \\
$\mathrm{~g}$ & 3 & Blob \\
$\mathrm{h}$ & 3 & 14 \\
$\mathrm{i}$ & 2 & 13 \\
$\mathrm{j}$ & 1 & Blob \\
$\mathrm{k}$ & 7 & 1 \\
$\mathrm{l}$ & 2 & 11 \\
$\mathrm{~m}$ & 1 & Blob \\
$\mathrm{n}$ & 1 & Blob \\
$\mathrm{o}$ & 8 & Blob \& 9 \\
$\mathrm{p}$ & 2 & 4 \& Blob \\
$\mathrm{q}$ & 1 & 1 \\
$\mathrm{r}$ & 1 & 3 \\
$\mathrm{~s}$ & 1 & 3 \\
$\mathrm{t}$ & 1 & 11 \\
$\mathrm{u}$ & 2 & 2 \\
\hline
\end{tabular}

B

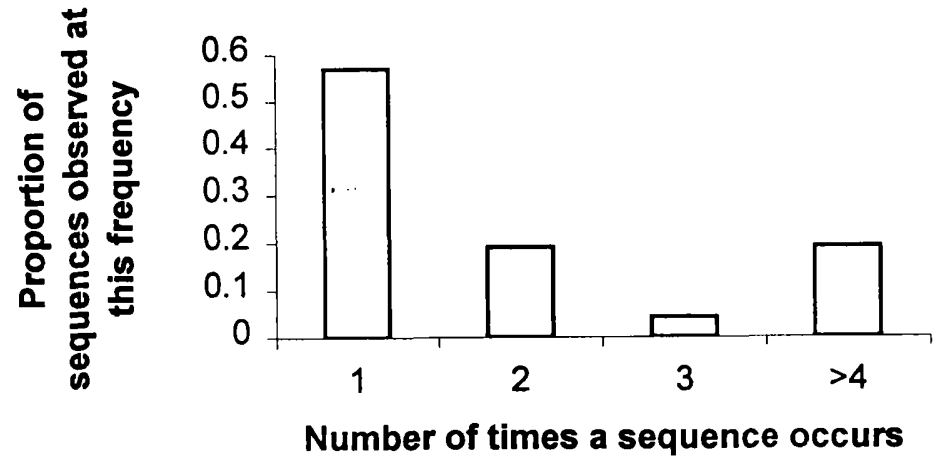


PCR, the frequency of the number of sequences found a given number of times was calculated (Clarke and Carbon, 1976). This showed that at the DNA level, the probability of finding a sequence once is greater than 0.5 , whereas the probability of finding a sequence multiple times is much lower, at less than 0.2 (Figure3.5).

The 21 3D7 stevor sequences were then compared with those sequences in the http://plasmodb.org/PlasmoDB.shtml database by BLAST analysis to determine on which chromosome they had been assigned. All the sequences were found on a chromosome with at least $95 \%$ identity to the database sequence. The stevor sequences from the clones obtained from the PCR reaction were distributed on all chromosomes with the exception of chromosomes 5 and 12 (Figure 3.5).

\subsection{Discussion}

The high polymorphism and copy number of Plasmodium multigene families makes it difficult to study them. As there are many copies of stevor in the parasite genome, it was essential to design primers that amplify the majority of genes with minimum primer bias. Primers were designed in relatively conserved regions flanking the region of highest polymorphism. Only products from the nested PCR reaction were analysed because this was the region with the highest diversity (only $33 \%$ similarity between sequences) (Kyes et al., 2001). These primers were also going to be used to analyse stevor transcription within single cells and as there is only a small amount of RNA, it is necessary to use a nested reaction to amplify products.

Both sets of primers used (external and nested) gave products of the expected size, with the slight size differences between the products being most likely due to size polymorphisms between the stevor genes. Sequence analysis of the nested PCR product showed that stevor genes were indeed amplified, while sequences from the closely related rif gene family were not amplified. This demonstrated the specificity of these primers for stevor. Individual clones were shown to hybridise to a single chromosome indicating the gene specificity of the nested products. Therefore the fact that the products from the nested PCR reaction hybridised to all 14 chromosomes of 3D7, demonstrated the universality of these primers. However, PCR bias makes it unlikely that every single stevor gene was amplified in a single PCR reaction and this was 
reflected in the hybridisation of PCR product with a slightly lower intensity to chromosomes 9 and 10.

With the primers used in this study 21 different stevor sequences were amplified and since all these sequences were found in the sequence databases we were able to assign the sequences to a chromosomal location. It is not unexpected that only 21 different stevor sequences were found although there are 33 different sequences in 3D7 as only 53 clones were sequenced. To have more than a $99 \%$ chance of identifying clones representing all 33 genes, assuming the primers are universal, it would require the sequencing of over 160 clones (Clarke and Carbon, 1976). Some of the sequences, for example a, $\mathrm{k}$ and o, were found to be amplified more than 5 times. Cheng et al., (1998) have already shown that some stevor amplify more efficiently than others, and the above results confirm this.

Comparison against the database showed that the stevor sequences identified in this study were distributed over most of the chromosomes except chromosomes 5 and 12. In all other respects, the distribution of stevor sequences was as expected with not more than 3 sequences per chromosome. Although the nested PCR product hybridised to all the chromosomes, not all stevor sequences were represented in the characterised clones. As only a limited number of clones were sequenced it is likely that for this reason no stevor genes from chromosomes 5 and 12 were identified. Alternatively, it is also possible that some of the sequences on the database have been have been wrongly assigned.

In conclusion, the primers designed were shown to be specific only for stevor genes. Furthermore, the primers amplified the majority of stevor genes in the $P$. falciparum genome, thereby allowing analysis of most if not all stevor genes from a single PCR reaction. Therefore, these primers allow a very specific and precise analysis of the different stevor genes and can be confidently used to analyse stevor at the transcriptional level. 


\section{CHAPTER FOUR}

\section{Analysis of stevor transcription}

\subsection{Introduction}

Antigenic variation in Plasmodium is mediated by switching gene expression between members of multigene families. Although there is some evidence to suggest that the expression of these genes is controlled at the transcriptional level, very little is known about the factors that influence switching and regulate control in Plasmodium. Switching expression of genes within these families allows the parasite not only to evade the immune system, but also to change cytoadherence properties in the case of $P$. falciparum and is therefore an important virulence mechanism used by the parasite. Thus, it is highly important to understand the mechanisms which control transcription within these multigene families.

An understanding of the timing of transcription of genes within a multigene family and the number of genes transcribed during the different stages of the parasite life cycle is important for elucidating the function of a multigene family. For example, the var genes are transcribed during the ring stage soon after merozoite invasion of the erythrocyte (Chen et al., 1998b; Kyes et al., 2000). This is significant as PfEMP-1 has a role in adhesion to endothelial receptors causing the $\mathrm{pRBC}$ to sequester during the parasite life cycle. This prevents clearance of the $\mathrm{pRBC}$ by the spleen that filters all $\mathrm{RBC}$ by recognising subtle changes in the rigidity of the $\mathrm{RBC}$ membrane. Scherf et al., (1998) have shown that only one predominant var transcript in pRBC. This is significant as PfEMP-1 is expressed on the surface of the RBC, and is therefore renders the cell exposed to and vulnerable to attack by the immune system. By allowing the immune system to see only a single variant form of PfEMP-1 at a time and then switching expression to another PfEMP-1 type, the parasite has a mechanism by which to evade the immune system. In the rodent malaria, $P$. yoelii, multiple Py 235 genes are expressed within a single schizont. However, each emerging merozoite is associated with only one Py235 product (Preiser et al., 1999). This protein is thought to have a role in merozoite selection of $\mathrm{RBC}$ and therefore as different Py235 proteins are expressed in different merozoites, if an immune response is made to one protein than 
the other merozoites could still successfully invade RBC. In addition, this may be an advantage as the protein is believed to be involved in invasion of different subsets of $\mathrm{RBC}$ utilising different receptors on the RBC. Transcription of $P y 235$ occurs during the schizont stages, consistent with the protein during the final stages of the asexual cycle.

With respect to control of transcription in a multigene family, the best characterised and understood example in the VSG genes of Trypanosoma brucei, a parasite which multiplies extracellularly in the blood stream of the host. Switching of VSG (see Section 1.4.3 for a detailed description) involves DNA rearrangements whereby a silent VSG gene is copied via gene conversion into an active expression site (Rudenko, 1999). However, this does not appear to be the principal mechanism for var gene switching in $P$. falciparum. There appears to be no defined var expression sites and all var genes can be expressed irrespective of their chromosomal location (Fischer et al., 1997). Regulation of var gene expression (Section 1.6.1), appears to be mediated at the transcriptional level when one promoter is activated while the others are silenced (Scherf et al., 1998). Silencing can be overcome by changes in chromatin states (Darkin-Rattray et al., 1996; Wolffe, 1996). Recently it has been shown that the 5' flanking region and intron sequence of var genes are responsible for the silencing of the var promoter (Deitsch et al., 2001). This indicates that co-operative DNA binding complexes and perhaps changes in chromatin structure are involved in the control of var gene transcription (Deitsch et al., 2001).

In P. falciparum members of the var, rif, and stevor multigene families are positioned next to each other in the subtelomeric regions of all the chromosomes (Bowman et al., 1999). As chromosomal topology surrounding such a region is thought to be involved in making the genes accessible to the transcription machinery, the chromosome structure surrounding an active var gene may also make the surrounding genes transcriptionally active.

Transcription of stevor has been detected in asexual stages (trophozoites and early schizonts) of the parasite (Cheng et al., 1998) as well as in gametocytes (Sutherland, 2001). However, a quantitative analysis of transcription has yet to be determined.

This chapter will use the primers characterised in chapter three to perform a detailed analysis of stevor transcription. The aims are to determine (1) the transcription profile of stevor in parasite cultures and in single micromanipulated parasites, (2) the 
timing of transcription, (3) the repertoire of genes transcribed and (4) whether there is a link between var and stevor transcription.

\subsection{Results}

\subsubsection{Not all stevor genes are transcribed during asexual blood stages}

Transcription of stevor was analysed by RT-PCR using total RNA obtained from an asynchronous parasite culture and both the external and internal sets of primers as described for the DNA analysis (Section 3.2.1). The products obtained from both reactions were separated on a $3 \%$ metaphor agarose gel (Figure 4.1 ). The products were of the expected size for both the external primers ( 600bp) and the internal primers $(\sim 300 \mathrm{bp})$. To get an ideas of how many different stevor genes were transcribed the radio-labelled nested RT-PCR product was used to probe a PFGE blot of separated $P$. falciparum 3D7 chromosomes. Clearly, the products from the nested reaction only hybridised to a subset of chromosomes (Figure 4.1BI) compared with the DNA product (Figure 4.1BII) suggesting that not all stevor genes are transcribed in vitro at the same level. Since only a limited PCR bias was observed during the DNA analysis of stevor (chapter 3), this indicates that not all genes are transcribed and this result is not due to a PCR bias. This is also shown as in total, using these primers 25 out of 33 stevor sequences were identified from 120 clones after PCR and RT-PCR.

\subsubsection{Repertoire of stevor transcripts in 3D7 parasites maintained in vitro}

To confirm that only a subset of stevor genes was transcribed, RT-PCR products were cloned and sequenced. To eliminate any possible PCR bias, products from 4 separate RT-PCR reactions (using RNA extracted from 3 different cultures) were cloned and 81 individual clones, several more than for DNA, were sequenced. The sequences were analysed using the ClustalX programme described in Section 3.2.4. In total 10 different stevor sequences were identified, demonstrating that although transcription within a culture is not restricted to a single stevor, only a subset of genes was transcribed. As previously described in section 3.2.3, in order to ensure that more than $99 \%$ of the genes present were detected it would require the sequencing of 165 clones. Since nearly half ( 81 clones) that amount of clones were sequenced and only 10 
A

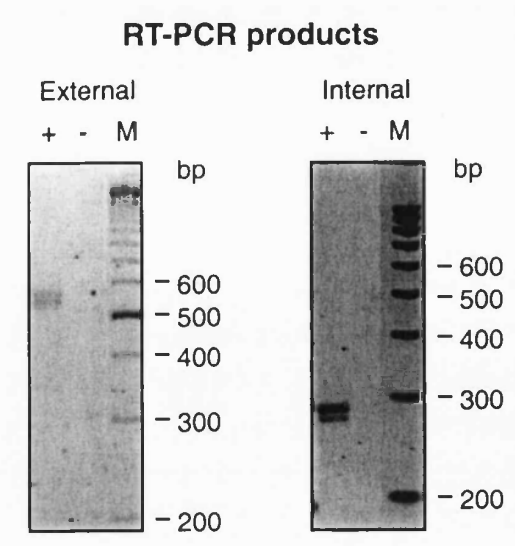

B

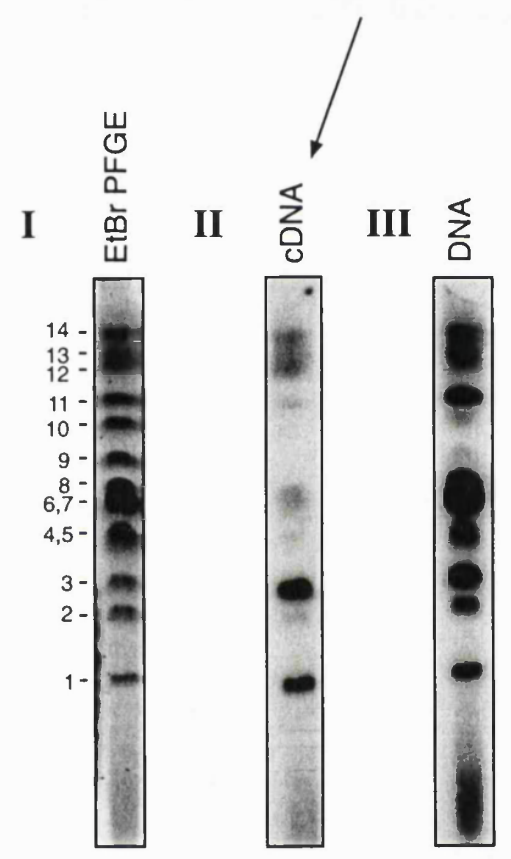

Figure 4.1 RT-PCR analysis of stevor. (A) cDNA products of $P$. falciparum 3D7 mRNA amplified with the external (smkf1 and smkf2) and internal (RepF1/F2 \& RepR) primers. $(+)$ with reverse transcriptase (-) without reverse transcriptase.

(B) Products of the nested RT-PCR reaction were then used to probe a Southern blot of P. falciparum 3D7 chromosomes. Chromosomes were resolved by PFGE and DNA from these gels was transferred onto nylon membranes and probed. I) PFGE of 3D7 resolved chromosomes stained with ethidium bromide. II) Probing with radiolabelled nested RTPCR product obtained from mRNA. III) Probing with radiolabelled nested PCR product obtained from DNA. 
unique sequences were identified, it would suggest that transcription is restricted to a subset of genes (at any one time). This study indicates that it is not the same subsets of genes that are transcribed each time.

Figure 4.2B shows the theoretical frequency of obtaining a transcript a certain number of times based on its frequency in the genome, and compares it to sequences obtained after PCR. Although several transcripts were found only once, in total there were fewer unique sequences even though more clones were sequenced from the RTPCR reactions. Also, the frequency of a sequence occurring in isolation was observed to be higher for DNA rather than RNA. However, the frequency of a sequence appearing several times is higher for RNA than DNA. Interestingly, one RT-PCR reaction amplified only a single type of sequence. While the possibility of PCR bias cannot be completely eliminated, the fact that it also was the dominant transcript in the other RT-PCR reactions would indicate that it is the dominant transcript in cultured parasites. This sequence located on chromosome 14, appeared in 46 out of 81 clones sequenced and was also found in the DNA analysis. The obtained cDNA sequences were also compared against sequences obtained from the database and to the sequences derived from the PCR of genomic DNA (Figure 4.2B). Four sequences (v, w, x, \& y) which were identified from sequencing RT-PCR cloned products were not previously described in the cloned products obtained from the PCR experiment, but were found to be present in the genomic sequence.

\subsubsection{Transcription of stevor occurs within an 8 hour window during the asexual parasite's 48 hour development cycle}

To determine the exact time of stevor transcription during the erythrocytic cycle a Northern blot analysis was performed. Total parasite RNA was extracted at two-hour intervals throughout the $48 \mathrm{~h}$ erythrocytic life cycle, from tightly $P$. falciparum cultures synchronised (as described in section 2.4.2). RNA from approximately an equal number of parasites from each time point were separated on a $1 \%$ agarose gel containing guanidinium thiocyanate (Kyes et al., 2000) and transferred to a nylon membrane (Figure 4.3B). Figure 4.3A also shows the corresponding morphological stages of parasite development at the time indicated. The immobilised RNA was then probed with radio-labelled nested stevor PCR products obtained from genomic DNA. 
Figure 4.2 (A) Comparison of DNA and cDNA sequences of cloned PCR products against sequences deposited in the $P$. falciparum http://plasmodb.org/PlasmoDB.shtml database. All unique sequences discovered are listed in column 1 . The number of times a sequence was found in the analysis and its chromosomal location are listed in columns 2 or 3 and 4 respectively. gDNA $=$ genomic DNA $c D N A=$ complementary DNA $n=$ number of clones (B) The probability of finding a particular stevor sequence is depicted by calculating the frequency distribution of stevor sequences amplified by PCR (white bars) and RT-PCR (black bars). 
A

\begin{tabular}{|c|c|c|c|}
\hline \multirow[t]{2}{*}{ Sequence type } & \multicolumn{3}{|c|}{ DNA source } \\
\hline & gDNA & cDNA & Chromosome \\
\hline a & 8 & 10 & 3 \\
\hline b & 1 & - & 3 \\
\hline c & 5 & 46 & 14 \\
\hline d & 1 & - & 14 \\
\hline $\mathrm{e}$ & 1 & - & 10 \\
\hline $\mathrm{f}$ & 1 & - & 14 \\
\hline g & 3 & 3 & Blob \\
\hline h & 3 & 2 & 14 \\
\hline $\mathrm{i}$ & 2 & - & 13 \\
\hline j & 1 & - & Blob \\
\hline $\mathrm{k}$ & 7 & 2 & 1 \\
\hline 1 & 2 & 4 & 11 \\
\hline $\mathrm{m}$ & 1 & - & Blob \\
\hline $\mathrm{n}$ & 1 & - & Blob \\
\hline o & 8 & - & Blob \& 9 \\
\hline $\mathrm{p}$ & 2 & - & $4 \&$ Blob \\
\hline$q$ & 1 & - & 1 \\
\hline $\mathrm{r}$ & 1 & - & 3 \\
\hline s & 1 & - & 3 \\
\hline $\mathrm{t}$ & 1 & - & 11 \\
\hline $\mathrm{u}$ & 2 & - & 2 \\
\hline $\mathrm{v}$ & & 2 & 12 \\
\hline w & & 8 & $9 \& 14$ \\
\hline $\mathrm{x}$ & & 1 & 3 \\
\hline $\mathrm{y}$ & & 2 & $9 \& 14$ \\
\hline
\end{tabular}

B

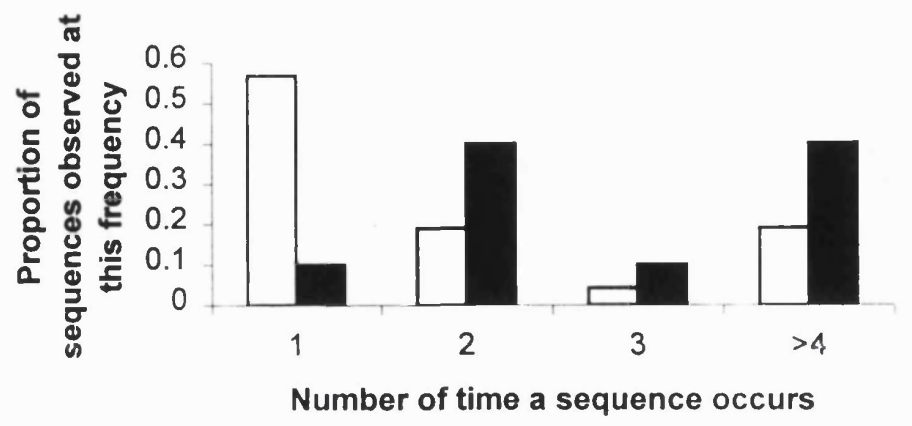


Figure 4.3 Timing of stevor transcription. P. falciparum 3D7 parasites were synchronised in vitro to initiate a bulk culture with merozoites. RNA was extracted from aliquots of the culture taken at $2 \mathrm{~h}$ intervals over one $48 \mathrm{~h}$ intra-erythrocytic developmental cycle. Time $(0 \mathrm{~h})$ corresponds to the time of erythrocyte invasion by merozoites. (A) Images from Giemsa-stained slides of parasites taken at the time points indicated (100X magnification). (B) Total RNA was electrophoresed through agarose, stained with ethidium bromide, transferred to nitrocellulose and hybridised with a radiolabelled DNA probe derived from stevor (C) P. falciparum control gene $p 40$ (D). Molecular mass markers is also shown. 


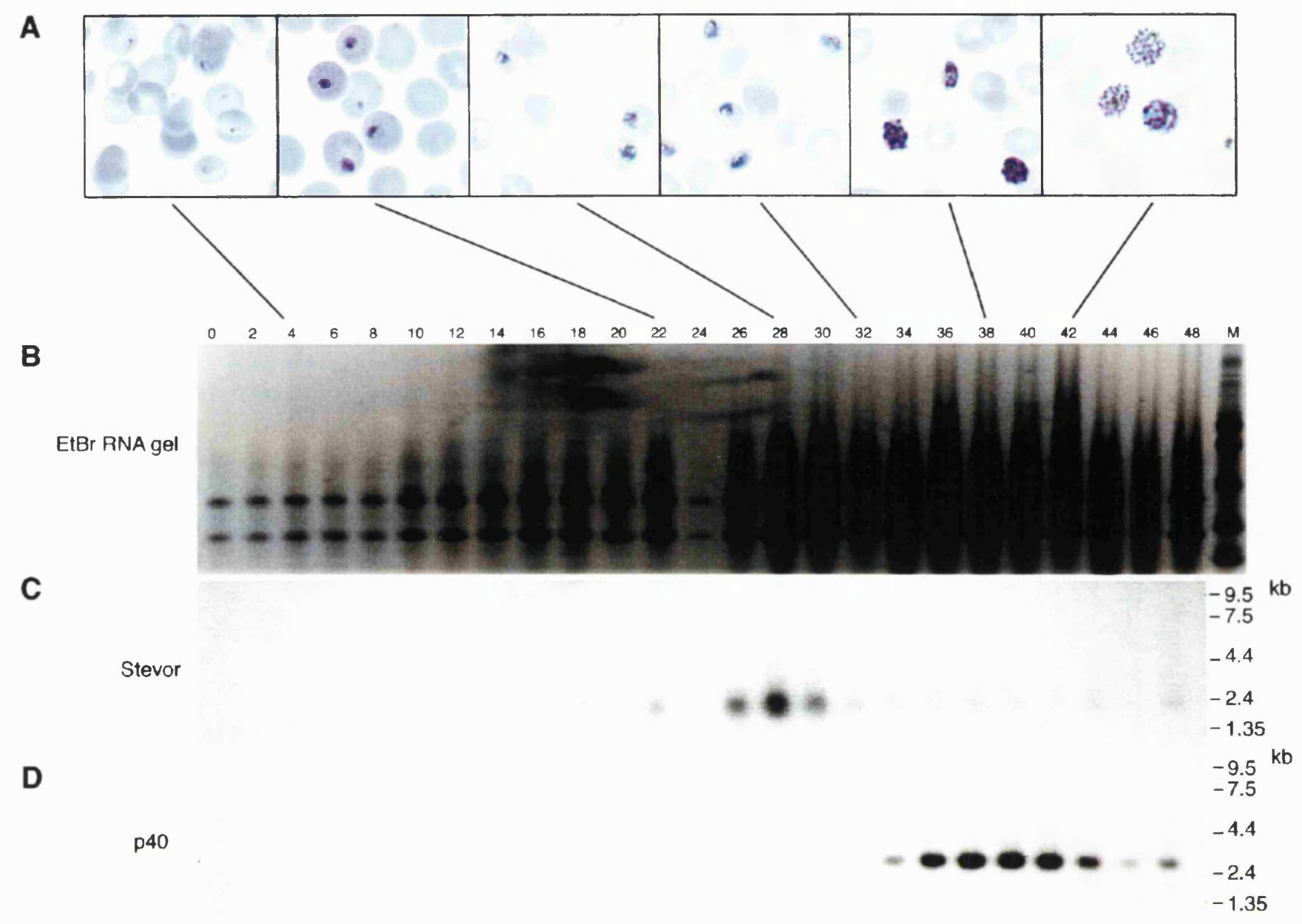


A diffuse band, corresponding to putative multiple stevor genes, of approximately $2.4 \mathrm{~Kb}$ was first detected in RNA prepared $22 \mathrm{~h}$ after parasite red cell invasion (Figure 4.3C). Maximum stevor transcription occurred at $28 \mathrm{~h}$ after which time the signal strength was rapidly lost. This was despite the fact that there is an increased amount of total RNA on the filter in the latter half of the life cycle, as seen on the EtBr stained gel (Figure 4.3B). Signal strength of the probe was greatest in mid- stage trophozoites indicating that stevor is actively transcribed at this stage. Probing of the same filter with $p 40$ (msp-7), that is specifically expressed in late trophozoites and schizonts, revealed a quite different transcription profile (Figure 4.3D) (Pachebat et al., 2001).

mRNA bands detected on the blot were approximately $2.4 \mathrm{~Kb}$ in size, although the size of the stevor coding sequence is $\sim 1 \mathrm{~kb}$. This is not an aberrant result as it is not uncommon to have both $3^{\prime}$ and $5^{\prime}$ untranslated regions (UTRs) as well as a poly(A) tail of $\sim 200 \mathrm{bp}$ at the $3^{\prime}$ end before the mRNA can be translated (Lewin, 1997). The diffuse bands that were observed on the membrane likely correspond to several different stevor transcripts, something already observed at the RT-PCR level.

Confirmation of the Northern blot data was obtained by the analysis of the time course RNA samples by nested RT-PCR. Similar results were obtained (Figure 4.4A) which indicated activation of stevor transcription after $22 \mathrm{~h}$ (Figure 4.4A). However, RT-PCR products were also detected throughout schizogony. This is probably due to the higher sensitivity of the RT-PCR assay compared to Northern blot analysis.

\subsubsection{Multiple stevor transcripts are detected in single micromanipulated parasites}

Multiple stevor transcripts were detected in RNA extracted from a population of cultured parasites. To establish whether this phenomenon was also reflected at the single parasite level, individual trophozoite-stage pRBC were selected by micromanipulation and analysed by RT-PCR for the presence of stevor transcript. Trophozoites around $28 \mathrm{~h}$ old were selected for RT-PCR analysis as this time point had been shown by the Northern blot analysis to be the time of maximum stevor transcription. A parasite culture was synchronised and the trophozoites collected after fractionation on a Sorbitol/Percoll gradient (Section 2.6.2) to enrich for trophozoites of the desired age.

Transcripts of stevor from micromanipulated, hence single parasites were detected after performing a nested PCR reaction and separation on a $3 \%$ metaphor 
agarose gel. RT-PCR products of the expected size (approximately $300 \mathrm{bp}$ ) were obtained from most of the single cells analysed (Fig 4.5). In addition, bands smaller than $300 \mathrm{bp}$ and in one cell (Fig 4.5, T4) a band larger than the expected size was observed. To establish whether these bands were related to stevor the RT-PCR products from these cells were cloned and sequenced. For reasons not known only clones with inserts of $\sim 300 \mathrm{bp}$ and below were generated and therefore it was not possible to analyse the larger products obtained during RT-PCR. Using the sequences obtained from the single cell clones to BLAST search the database confirmed that all the products were stevor derived. The smaller bands correspond to stevor products in which mispriming of one of the nested PCR primers has led to a truncated transcript being detected, while the $\sim 300 \mathrm{bp}$ fragments contain the full-length expected stevor sequences. While it is not clear whether the truncated stevor products obtained are a RT-PCR artefact or represent real transcripts, more than one stevor product can be detected per cell, indicating that more than one gene is transcribed.

\subsubsection{Transcription of stevor is not directly linked to var gene expression}

It has been suggested that changes in chromatin structure control the transcription of genes within multigene families (Wolffe, 1996). Previous work has shown that $\mathrm{pRBC}$ selected on defined cytoadherance receptors such as CSA, CD36 or ICAM-1 express a dominant var (Scherf et al., 1998). Although ubiquitous transcription of all var variants appears to occur in early ring stages, only a single transcript is detected in the trophozoite-stage parasite (Taylor et al., 2000a), suggesting a silencing mechanism where only one site is accessible to the appropriate transcription factors. In $P$. falciparum members of the var, rif, pf60 and stevor multigene families are located together in the subtelomeric regions of all the chromosomes (Figueiredo et al., 2000; Bowman et al., 1999). To assess if there is a link between stevor and var transcription, trophozoites were selected on the cytoadherence ligands CSA, ICAM-1, and CD36 (Section 2.9). RNA was extracted from parasites that had bound to these ligands or unselected parasites and used for RT-PCR. RT-PCR using RNA from at least two separate experiments gave products of the expected size for stevor (Figure 4.6A). Comparison of the bands obtained for each experiment indicate that differences in the transcription pattern exists between the different samples. 
A

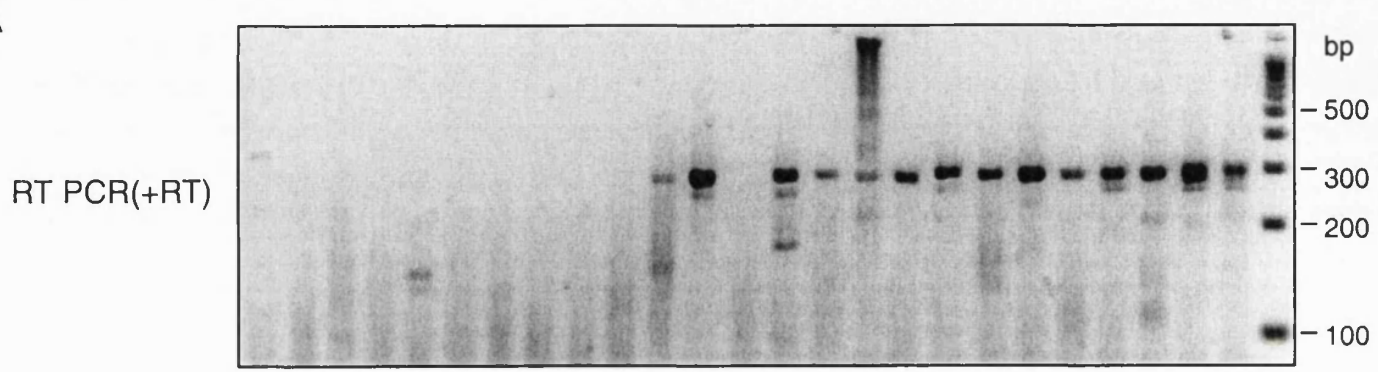

B

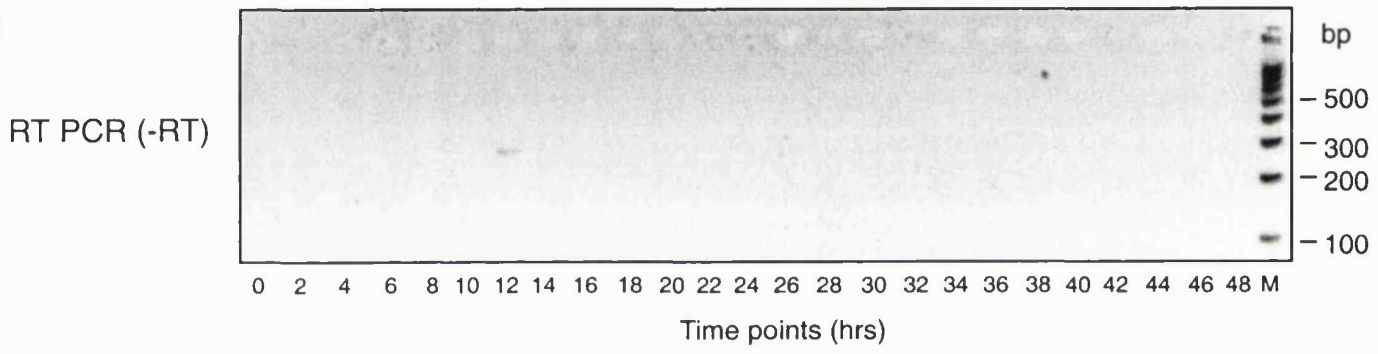

Figure 4.4 RT-PCR analysis of RNA samples taken at varying times throughout the parasite life-cycle in the presence (A) and absence (B) of reverse transcriptase as a control. 


\section{Trophozoites}

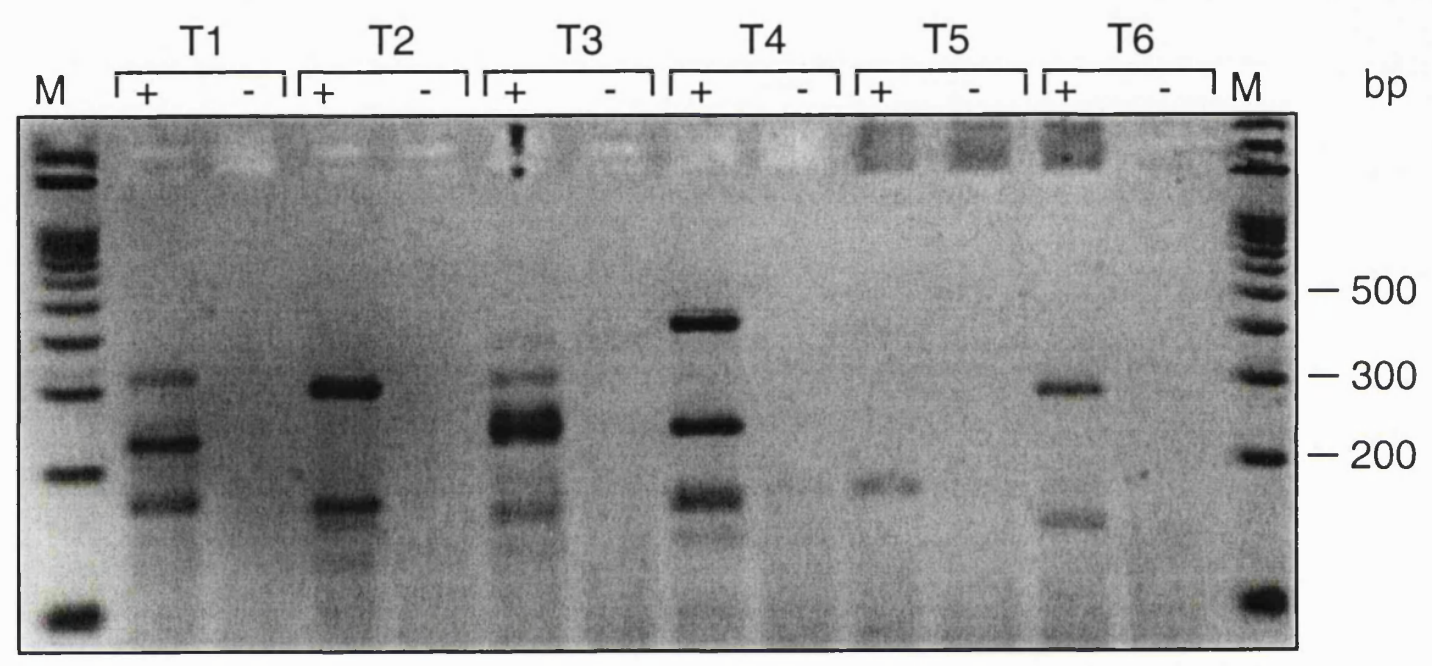

Figure 4.5 RT- PCR of single cell micromanipulated trophozoites. A metaphor agarose gel (3\%) stained with ethidium bromide showing the nested RT-PCR products from 6 individually micromanipulated trophozoites (T1-T6). (+) with reverse transcriptase and (-) without reverse transcriptase is shown here. 
To analyse the differences between the selected and unselected parasites in more detail, the RT-PCR products were labelled and used to probe chromosomal blots (Figure 4.6B). There are clear differences in the hybridisation pattern observed for all the samples analysed. Differences did not just exist between the samples obtained by selection on different receptors (Fig 4.6B), but also in samples selected on the same receptor (compare Fig 4.6B, 1\&2). While there was some similarity in the pattern seen for some of the samples analysed (Fig 4.6B, ICAM, 1\&2), this was not consistent. On closer inspection the hybridisation intensities were different for chromosomes 2 and 3 between the two experiments and hybridisation to chromosome 13 was only detected in experiment 1 . Therefore, the similarity most likely represented chance occurrences. The results obtained here contrast with what would have been expected for var (only one transcript)(Scherf et al., 1998) and strongly suggest that transcription of var and stevor is not directly linked. 
Figure 4.6 Analysis of stevor transcription by RT-PCR and Southern blot using DNA from unselected parasites or parasites selected on different ligands. A metaphor agarose gel (3\%) was used to resolve the RT-PCR products of $P$. falciparum 3D7 mRNA amplified with smkf1 and smkf2 and RepF1/F2 \& RepR (only the internal reaction is shown). (A) RT-PCR products obtained from mRNA of unselected and either CD36-, CSA- or ICAM-1-selected parasites after a nested PCR reaction using stevor-specific primers. Lanes 1 and 2 represent products obtained from two independent experiments. $M=100$ bp ladder. (B) Probing of a Southern blot of 3D7 chromosomes resolved by PFGE using stevor-specific RT-PCR products from (A). The positions of the different chromosomes are indicated. $(+)$ with reverse transcriptase $(-)$ without reverse transcriptase. 

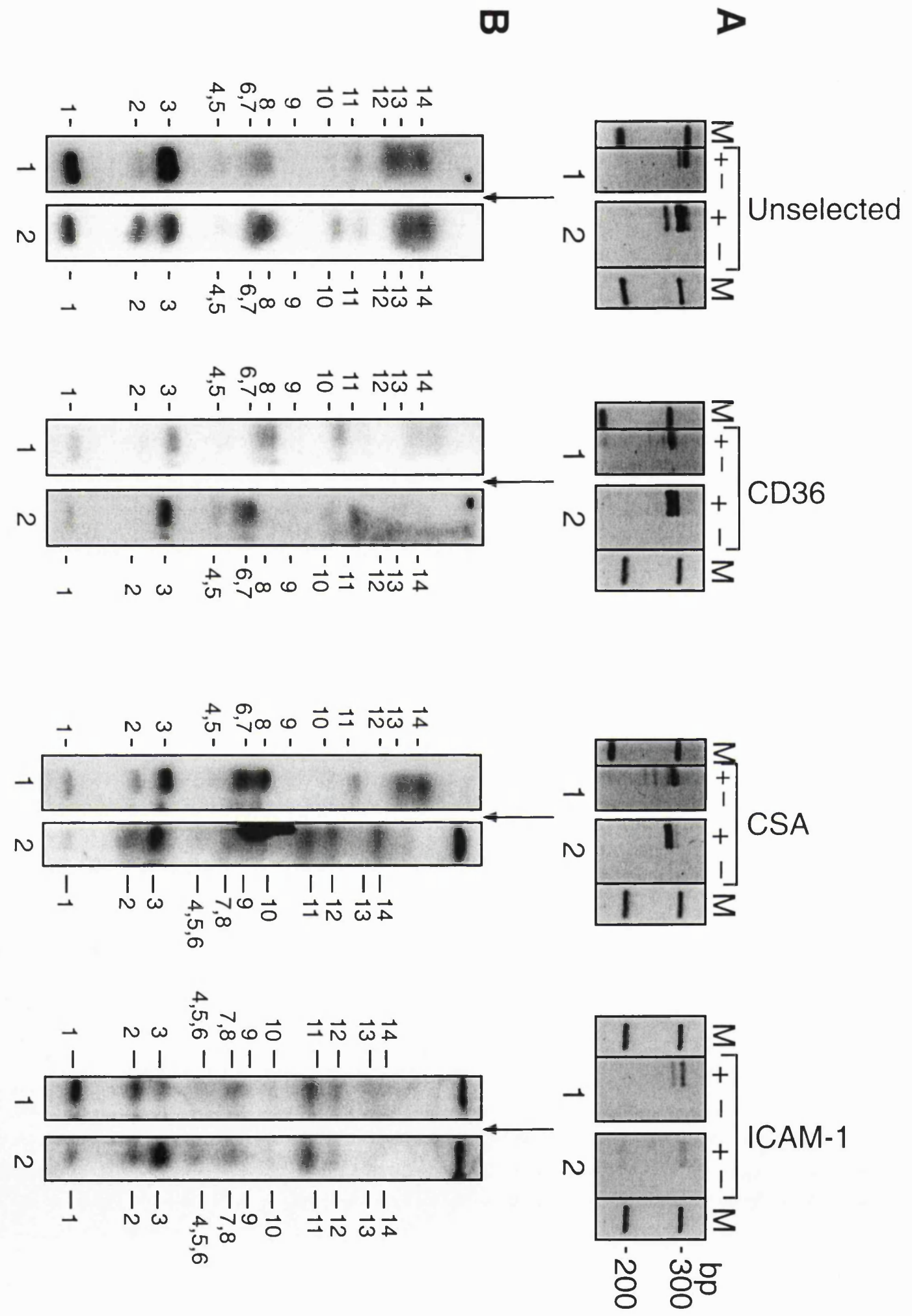


\subsection{Discussion}

Using both RT-PCR and Northern blot analysis, this study has shown that stevor is transcribed in the asexual stages of the parasite in a tightly regulated fashion. Specifically, stevor is transcribed at mid-trophozoite stage with maximal expression before nuclear division. This is in accordance with earlier work that demonstrated transcription of stevor during trophozoite and early schizont stages by RT-PCR (Cheng et al., 1998). Although the RT-PCR results shown here detected the presence of stevor transcripts throughout schizogony, this is likely to be due to the increased sensitivity of nested RT-PCR reactions. Previous attempts by another group at detecting the timing of stevor transcription by Northern blot analysis were unsuccessful (Cheng et al., 1998) due to low levels of stevor mRNA. Although a clear result was obtained in the current investigation by Northern blot analysis, signal strength was very weak, indicating that stevor is transcribed at low levels. Previous work has shown that both var (ring stage) and rif(late ring/early trophozoite stages) are transcribed and expressed for only a short time during erythrocytic parasite maturation (Kyes et al., 2000) and this has been also been demonstrated in this investigation for stevor. Comparing the relative timing of transcription for the different multigene families, gives some indication that stevor is transcribed independently from var and rif. Overall there seems to be sequential pattern of transcription with var being transcribed earliest followed by rif and finally stevor. Whether the timing of transcription is linked to protein expression and therefore indicates important functional differences between the proteins encoded by these multigene families still needs to be established.

The stevor multigene family is located on all chromosomes and the primers used in this study were shown to amplify most of stevor as confirmed by Southern blot analysis. Using these primers it was demonstrated that only a subset of the genes is transcribed in a parasite population and this corroborates earlier findings (Cheng et al., 1998). Interestingly, the expression pattern differs between cultures indicating that it is not the same subset of genes that are transcribed each time. The fact that the transcription profile changes over time may indicate some similarity to what is observed for the var genes where $\sim 2 \%$ of parasites in culture switch antigenic phenotypes in the absence immune pressure (Roberts et al., 1992). This could be clarified by taking 
samples sequentially from a long term $P$. falciparum culture to determine if there are specific subsets of stevor to potentially elucidate the rate of switching. Clearly though, there appears to be some kind of mechanism that results in transcriptional exclusion which seems to be acting, as this study showed that even in long term cultured parasites only subsets of stevor are expressed. It is not yet clear from this work whether there are sets of stevor that are always expressed together and whether this is functionally important.

The subtelomeric regions of the chromosomes of malaria parasites seem to be the preferential location of genes involved in antigenic and phenotypic variation. It has been proposed that this allows the preferential creation of new variants by recombination (Freitas-Junior et al., 2000; Taylor et al., 2000b). Changes in the chromatin structure surrounding the subtelomeric region are thought to be involved in making the genes accessible to the transcription machinery and therefore influence gene activation and silencing (Darkin-Rattray et al., 1996; Wolffe, 1996). It is therefore possible that the chromosome structure surrounding an active var gene may make the closely positioned genes also transcriptionally active, which would result in expression of genes on the same chromosome.

The proximity of individual copies of stevor, rif and var on a chromosome (Figueiredo et al., 2000) and also the fact that they are transcribed sequentially raises the interesting possibility that expression of individual members of this family maybe linked. The chromatin structure around an active var gene might also allow the transcription of other genes at and around that site. Previous work has shown that individual parasites only express a single full-length var gene indicating the transcriptional activation of a single subtelomeric region (Scherf et al., 1998). To assess whether the expression of a single var gene also restricted transcription of stevor to the same chromosomal region parasites were selected on cytoadherence ligands for expression of specific var genes. In neither case was it possible to establish a direct link between the types and chromosomal location of transcriptionally active stevor and the active var locus. Preliminary experiments studying a link between var and rif genes also failed to establish a link between transcription of these genes (Kyes et al., 2001).

In all experiments stevor seems to be transcribed from several chromosomes and in a random fashion as has been observed for unselected parasites. However, these results may not be that surprising in light of opposing findings which indicate that mature trophozoites express multiple full-length var and selection occurs at the 
expression level with a dominant PfEMP-1 molecule expressed. This suggests that a defined adhesive phenotype can be associated with expression of multiple var genes (Noviyanti et al., 2001). Moreover, recent findings indicate that var gene silencing is regulated by the var intron and therefore would not be expected to have global effects on the whole subtelomeric region of a chromosome (Deitsch et al., 2001). Further evidence for the absence of any link between var and stevor transcription came from the observations made in this study that single micro-manipulated parasites transcribe more then one member of stevor. This was indicative of more then one subtelomeric region being transcriptionally active in most instances. Although there are lots of var genes transcribed, most of these are truncated transcripts (Taylor et al., 2000a), and this could also be true for stevor transcripts as this study did not analyse the full gene. Nevertheless, it is unlikely that the stevor transcripts are truncated as analysis of fulllength transcripts have been carried out by other groups and they have also shown that multiple full-length stevor are transcribed by parasites in culture (Cheng et al., 1998; Sutherland, 2001). Although more than one stevor is transcribed, this does not mean that all the transcripts are translated. Therefore there may be another level of control of stevor as suggested by the expression of PfEMP-1 (Noviyanti et al., 2001) whereby, several genes are transcribed but only one gene is expressed at a given time. Alternatively, the function of the protein (STEVOR) encoded by stevor genes may be such that multiple genes need to be expressed. Finally, products generated from selected parasites have yet to be cloned and sequenced to establish whether there are any differences in the genes transcribed between parasites selected on different ligands. If a pattern is observed, it may suggest that the function of the protein is associated with the adherence properties of the parasite. 


\section{CHAPTER FIVE}

\section{Subcellular characterisation and localisation of STEVOR in $P$. falciparum infected erythrocytes}

\subsection{Introduction}

The intracellular development of malaria parasites requires modification of the red cell surface to allow for parasite growth. Parasite proteins must therefore be transported across the parasite membrane and PVM before reaching their final destination in the host cell cytosol, exposed on its surface and/or to be secreted. The $\mathrm{RBC}$ is anucleated and does not synthesise and transport proteins. Consequently, the parasite needs to induce modifications in the host cell in order to transport proteins and these are evident in the host cell early in the ring and trophozoite stages (Elford et al., 1995).

Very little is known about the transport of proteins by the parasite into the red cell cytosol and to the RBC membrane. High resolution fluorescence microscopy studies have demonstrated that discrete tubules and vesicles present in the infected erythrocyte cytoplasm form an interconnected tubo-vesicular network that extends from the PVM to the red cell membrane (Etzion and Perkins, 1989). It has been proposed that cytoplasmic vesicles are involved in the intracellular trafficking of parasite proteins to the host cell membrane (Barnwell, 1990). Other antigens are believed to be transported to the RBC plasma membrane and surface of the $\mathrm{RBC}$ via structures called Maurer's clefts (MC) (Hinterberg et al., 1994). MC are long slender membrane bound vesicles that extend from the parasite and surrounding PVM, into the RBC cytoplasm and some appear to fuse with the erythrocyte membrane (Atkinson and Aikawa, 1990). They are detected in $P$. falciparum infected RBC as early as $4 \mathrm{~h}$ post invasion and maintained until after host cell rupture (Aikawa et al., 1986; Hui and Siddiqui, 1988). There is evidence to suggest that MC are not freely diffusible within the red cell cytoplasm but are associated with the red cell plasma membrane or cytoskeleton possibly via a resident $\mathrm{MC}$ protein called $P$. falciparum skeletal binding protein (PfSBP)-1 (Blisnick et al., 2000). Proteins with an apparent molecular mass of 45-50 $\mathrm{kDa}$ have also been localised in the MC (Etzion and Perkins, 1989; Hui and Siddiqui, 
1988; Martinez et al., 1998) but as yet their function is unknown. In addition, a very large P. falciparum protein, Pf332, has been shown to locate to the Maurer's clefs (Hinterberg et al., 1994).

Recent experiments have shown that both PfEMP-3 and PfEMP-1 traffic through the MC (Waterkeyn et al., 2000). PfEMP1, found on the surface of the RBC associates with a protein called KAHRP, which is located in electron dense knob like structures under the RBC membrane (Section 1.6.3.1). Work on the transport of KAHRP has suggested that the MC may also be a pre-assembly and sorting point for proteins on their way to the RBC surface (Wickham et al., 2001). Earlier electron microscopy work has shown that MC are associated with electron dense material in knob positive parasite, and this is not observed in knob minus (K-) parasites (Aikawa et al., 1986) suggesting a role in transport of proteins to knob complexes. MC are also found in gametocytes (Hui and Siddiqui, 1988) and may have a similar role of transporting parasite proteins to the RBC surface in these stages.

The proteins encoded by multigene families have different subcellular locations and this is reflective of their function. PfEMP-1 is located on the RBC surface and as discussed above, is involved in evasion of the host immune system as it is antigenically variant. Furthermore, PfEMP-1 causes $\mathrm{pRBC}$ to sequester in the brain and in the placenta and is involved in rosetting (see Section 1.6.2).

The proteins (p235) encoded by the Py235 multigene family of the rodent malaria $P$. yoelii, are localised to the rhoptries. As described earlier (Section 1.5) this protein has been shown to be involved in binding to red blood cells of all ages, indicating a possible role in red-cell binding of the merozoite (Ogun and Holder, 1996). Changes in phenotype from a virulent to an avirulent type of infection after the passive transfer of antibodies against p235 (Freeman et al., 1980) suggest that the variant properties of Py235 may be related to its possible functions of binding to different receptors.

Although antigenically variant proteins have been implicated in the virulence of the parasite and associated with malaria pathology, the host antibody responses to these proteins can be protective. As mentioned in the Introduction, his has been clearly demonstrated for proteins encoded by the var gene family. Studies by various groups have shown that antibody against a spectrum of variant PfEMP-1 antigenic types is a significant component of the host-protective immune response in man (Bull et al., 1998; Marsh et al., 1989). Furthermore, antibodies are raised against other antigenically 
variant proteins such as RIFINs and vir, in natural infections, although it has not yet been determined whether these responses are protective (Kyes et al., 1999; del Portillo et al., 2001; Fernandez et al., 1999).

Cheng et al., (1998) predicted STEVOR to be membrane bound proteins very similar in structure to RIFINs. The predicted protein structures for both STEVOR and RIFIN share a similar highly positively charged segment with PfEMP-1 immediately inside the predicted C-terminal cytoplasmic domain and therefore all three may be anchored similarly in the red cell surface. Not all the proteins involved in sequestration and rossetting are known. The predicted size structure for STEVOR and the RIFINs is equivalent in size to the rosettins, small polypeptides on the cell surface that mediate rosetting (Helmby et al., 1993), suggesting they might have a possible role in rosette formation (Kyes et al., 1999). Although the RIFINs play an accessory role in rosetting, this is not believed to be their primary function as several cloned parasite lines that do not rosette express these genes (Kyes et al., 1999). Finally, PfEMP-1 is not found on the surface of the late stage gametocytes which are also known to sequester (Piper et al., 1999). As STEVOR is transcribed in gametocytes, it is possible that it might have similar properties to PfEMP-1 and therefore may play a role in sequestration at this stage.

In view of the above, it is important to investigate the location of STEVOR as this will help elucidate its function and hence its possible involvement in immune evasion, virulence and pathology of $P$. falciparum malaria. This chapter describes the generation of antibodies against STEVOR and uses them to determine the molecular size and the timing of expression of STEVOR and the location of STEVOR within the pRBC.

\subsection{Results}

\subsubsection{Design of STEVOR peptides in order to generate antibodies}

To characterise STEVOR, antibodies were generated against several different STEVOR peptides. Due to the high degree of polymorphism between STEVOR sequences, peptides were designed corresponding to the most conserved regions of STEVOR. These regions have been proposed to lie on the cytoplasmic side of the RBC 


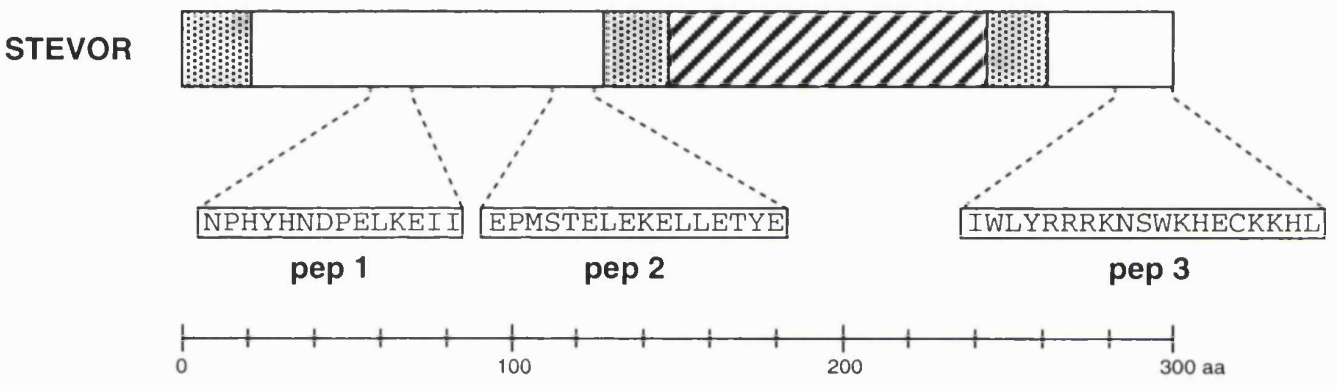

Figure 5.1 Production of $\alpha$-STEVOR antibodies-peptide design. A schematic showing the position of the three peptides (pep1, pep2, pep3) used to generate STEVORspecific antibodies. (I) putative transmembrane domains. All three peptide sequences were designed within conserved and putative cytoplasmic domains. (/) highly polymorphic region. 
membrane and therefore unlikely to be exposed on the surface of the $\mathrm{pRBC}$ (Cheng et al., 1998). From the work described in the previous two chapters, there is some evidence that only a limited set of STEVOR genes are transcribed in culture (Chapter 4), bit up to now there was no evidence as to which transcripts were translated. Therefore, three peptide sequences (peptide 1-3) were designed to regions of high sequence homology across six published full-length sequences of STEVOR (Figure 5.1) (Cheng et al., 1998). Peptides 1 and 2 are in close proximity to each other and are located near the amino terminal end of the protein. The third peptide was designed on a conserved region located at the carboxy terminal end of the protein (Figure 5.1).

Since hydrophilicity is a positive indication of antigenicity, the peptide sequences were checked for hydrophilicity using the Protean ${ }^{\circledR}$ programme. This indicated that all the peptides were suitable to use for antibody generation. The designed peptide sequences were compared against those in the NCBI $P$. falciparum database to ensure that they were specific for STEVOR and not the closely related RIFIN family. Polyclonal sera were raised against the peptides, following immunisation of both rabbits and mice (Section 2.26) and used to localise and characterise STEVOR.

\subsubsection{Characterisation of anti-STEVOR antibodies using ELISA}

The specificity of the anti-STEVOR antibodies generated in mice and rabbits were firstly analysed by ELISA.

The sera from the two rabbits (6344 and 6346) which had been immunised with peptide 1 were tested. Pre-immune serum from both rabbits did not bind to peptide 1 , while the immune serum gave a positive result reacting up to a serum dilution of 1/10240. (Figure 5.2A). To eliminate the possibility of non-specific binding to either PBS (used to dilute the peptide) or BSA (used in the dilution buffer for the serum), controls for immune serum binding to PBS and BSA were performed. The results were negative indicating that there was no non-specific binding (Figure 5.2A immune without peptide). As a positive control for the assay, two wells on the plate were coated with peptide conjugated to $\mathrm{KLH}$, which acts as a universal carrier (Janeway et al., 1999). Serum from individual animals from the group of mice immunised with peptide 1 were also tested by ELISA (Figure5.2B). All mice had high titres of antibody specific for peptide 1, with mouse 3 and mouse 4 having the highest titres of antibody. 
Figure 5.2 An ELISA to determine the presence of anti-peptide 1 antibodies in sera from (A) rabbits and (B) mice immunised with STEVOR peptide 1. The serum was diluted using a 2-fold serial starting at 1:10. Two rabbits (44 and 46) were immunised and a group of 4 mice. Rabbit pre-immune (PI) and normal (norm) mouse serum were used as negative controls. $\mathrm{I}=$ immune $\mathrm{rb}=$ rabbit $\mathrm{ms}=$ mouse. To test for non-specific binding of the serum, the serum was tested against wells coated without peptide (rb 44 no pep). 
A

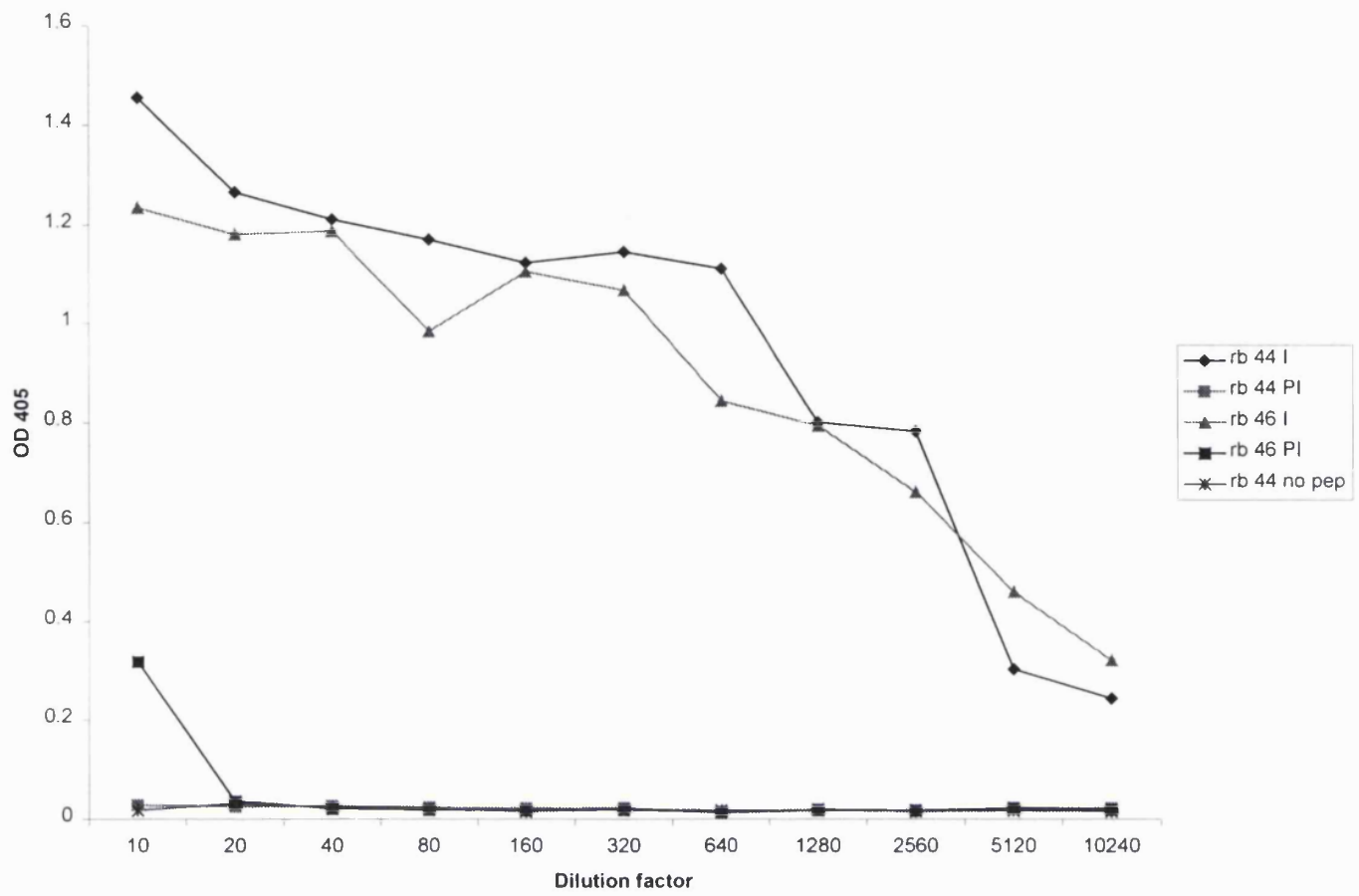

B

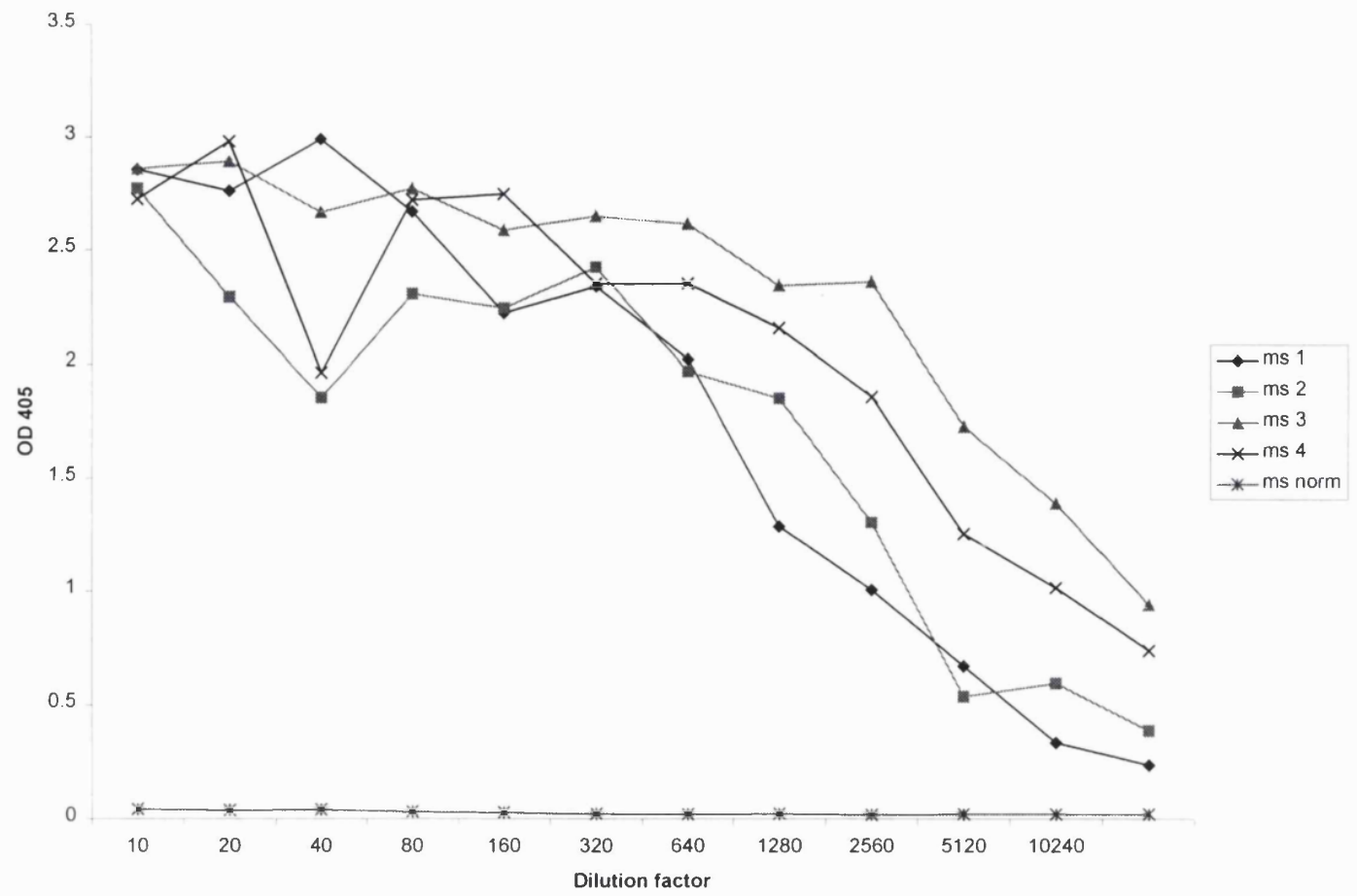


The same approach was used to test antibody reactivity against peptides 2 and 3 . Rabbit serum against peptide 2 from both rabbits gave high titres (Figure 5.3A) but the sera from mice immunised with peptide 2 had low titres even after the third boost (Figure 5.3B). Antibodies against peptide 3 were only raised in mice and ELISA results demonstrated that high titres of antibody were generated in all mice against this peptide even after the first boost (Figure 5.4).

\subsubsection{Anti-STEVOR antibodies recognize a protein of $\sim 37 \mathrm{kDa}$}

To determine whether the immune serum generated in mice and rabbits against the STEVOR peptides bound specifically to parasite proteins and to investigate the size of the protein recognised, a Western blot analysis was performed. TX-100 detergent, which is non-ionic, only solubilises some proteins while SDS is an ionic detergent that solubilises many of the proteins not soluble in TX-100 buffer. Parasite proteins were thus characterised as TX-100 soluble or SDS soluble as both extraction buffers were used sequentially to prepare schizont protein extracts. The protein extractions were separated on a pre-cast $4-12 \%$ Bis/Tris gradient gel under reducing conditions and after transfer of the proteins onto a membrane, were probed with anti-peptide 1 pre-immune and immune rabbit serum. Bound antibody was detected using an enhanced chemiluminescent substrate which is extremely sensitive. No difference was observed between the pre-immune and immune serum in their ability to bind to the schizont extracts indicating a high level of non-specific binding (Figure 5.5B).

To overcome the difficulty of non-specific binding of antibodies to the parasite an attempt was made to purify STEVOR specific antibodies against peptide 1 (see section 2.24.3). Affinity purification of the serum to make it specific for the peptide meant incubating the serum with the peptide and then eluting off the bound antibody. It was therefore necessary to conjugate the peptide to a high molecular weight protein in order to run it on the gel. Maleimide activated BSA, which is a high molecular weight protein, was used as a conjugate for peptide 1 since $\mathrm{KLH}$ had been used for generation of the immune serum. To determine if the immune serum bound to the conjugated peptide and not to BSA, the conjugated protein as well as BSA alone were run on a gel and transferred to a membrane for Western blotting (Figure 5.5C). The immune serum 
Figure 5.3 An ELISA to determine the presence of anti-peptide 2 antibodies in sera from (A) rabbits and (B) mice immunised with STEVOR peptide 2. The serum was diluted using a 2-fold serial dilution starting at 1:10. Two rabbits (2406 and 756) were immunised and a group of 4 mice. Rabbit pre-immune (PI) and normal (norm) mouse sera were used as negative controls. $\mathrm{I}=$ immune $\mathrm{rb}=$ rabbit $\mathrm{ms}=$ mouse 
A

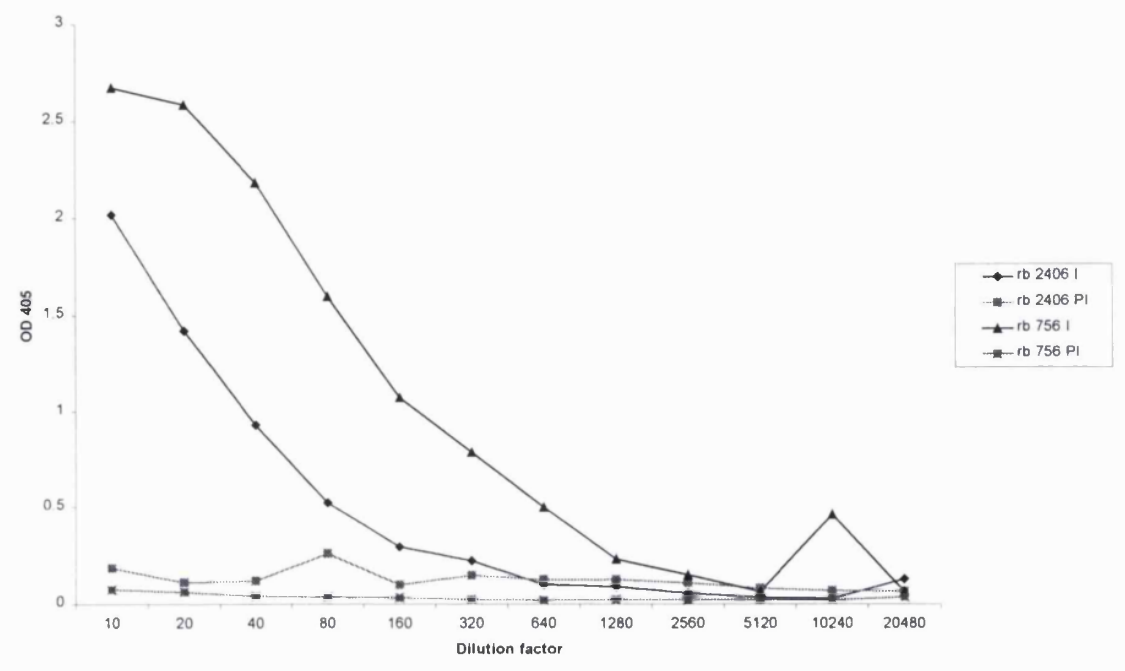

B

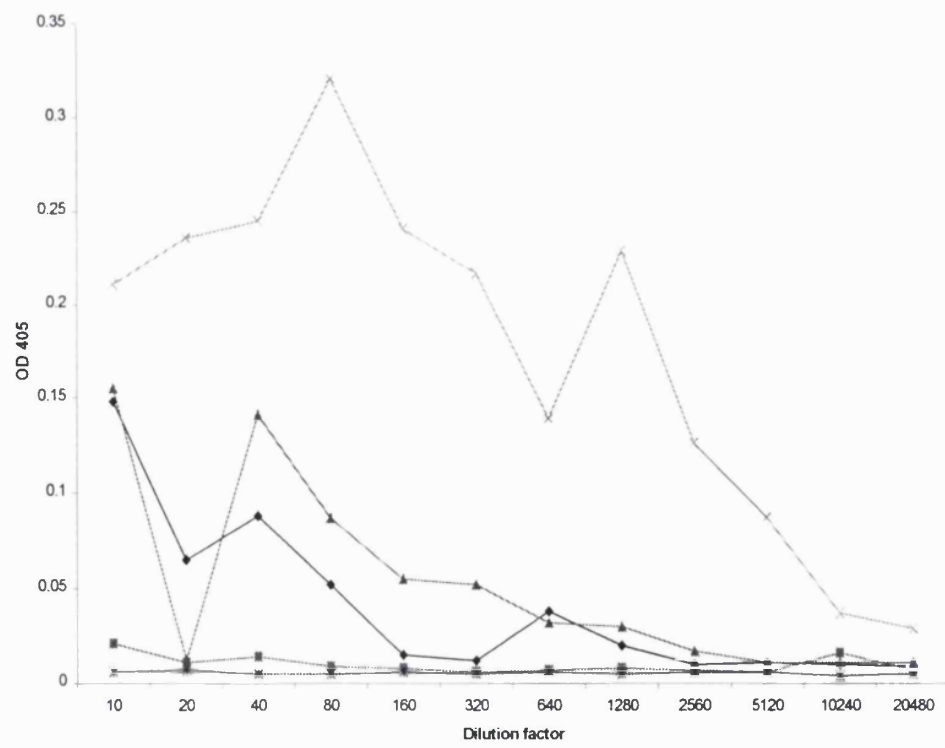




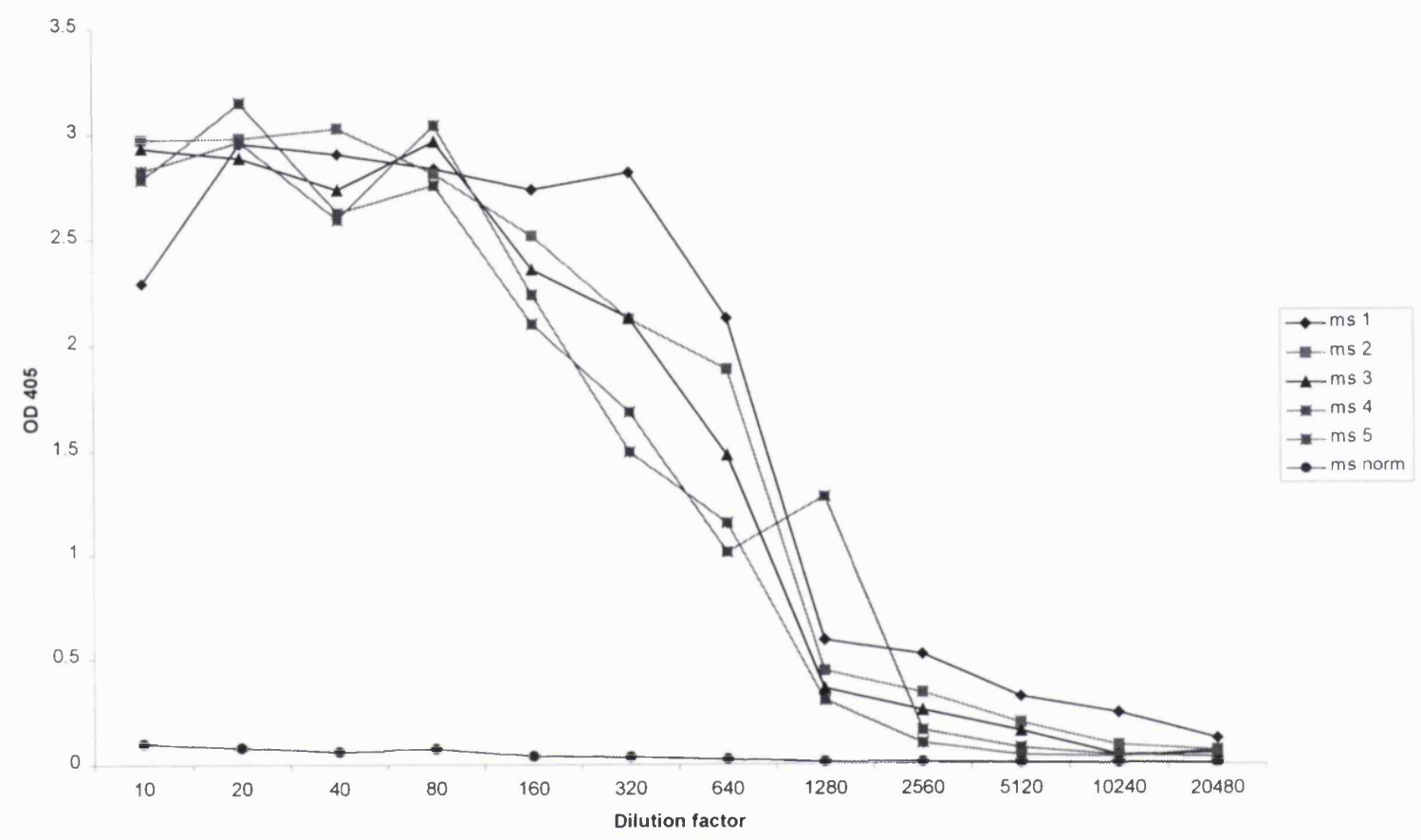

Figure 5.4 An ELISA to determine the presence of anti-peptide 3 antibodies in sera from mice immunised with STEVOR peptide 3. The serum was diluted using a 2-fold serial dilution starting at 1:10. A group of 5 mice were used. $\mathrm{ms}=$ mouse norm $=$ normal 
bound to the peptide 1 conjugated to BSA and not to BSA alone whereas the preimmune serum did not bind to either BSA or BSA conjugated peptide 1 (Figure 5.5D). What appears to be binding to BSA alone (Figure 5.5D2a) is non-specific binding, as there is clearly no protein on that region of the membrane according to the Coomassie stained gel (Figure 5.5Ca). These results confirmed that only anti-peptide 1 antibodies bound to the BSA conjugated peptide and hence it was used for affinity purification.

The immune and pre-immune sera were incubated with membrane bound BSA conjugated to peptide 1 , and subsequently the bound antibodies were eluted. The eluted fraction was then used to analyse TX100 and SDS extracts of schizont proteins. Comparison of whole immune serum, affinity purified immune serum and pre-immune serum showed no difference in their ability to bind schizont extracts indicating that some degree of non-specific binding still remained (results not shown). This may be due to the binding of non-specific antibodies to the BSA-peptide conjugate, or it is also possible that the washes before elution of the antibody were not stringent enough, resulting in the presence of remaining non-specific antibodies in the eluted fraction.

The results of the Western blot analysis were inconclusive, even after affinity purification of the anti-peptide 1 serum, and therefore an immunoprecipitation was performed. An asynchronous P. falciparum 3D7 culture was labelled using ${ }^{35} \mathrm{~S}$ methionine/cysteine and proteins were extracted using both TX-100 and SDS. The protein extracts were first pre-cleared for non-specific binding by incubating them with pre-immune sera (see section 2.31). An immunoprecipitation experiment was then performed using the pre-cleared extracts and rabbit anti-peptide 1 immune and preimmune sera. A unique band of $\sim 37 \mathrm{kDa}$ was detected by the anti-peptide 1 immune sera but not by the pre-immune sera in the SDS fraction of proteins (Figure 5.6A2b). A Western blot using serum from a group of mice immunised with peptide 1 also gave the same result (Figure 5.6B).

Western blot analysis was performed using anti-peptide 2 antibodies from rabbit 756 as it had the highest antibody titres. As affinity purification of the serum did not eliminate the non-specific binding from the pre-immune serum when performing a Western analysis using anti-peptide 1 serum, a new method was used to eliminate nonspecific binding by incubating the serum with RBC lysate. However, again there was no visible difference between the blots probed with the pre-immune or immune anti-peptide 2 sera (Figure 5.7A). Therefore, an immunoprecipitation experiment was 
A

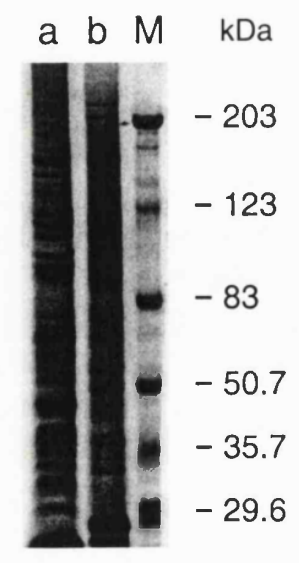

C

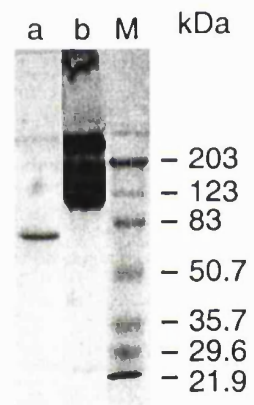

B

$\frac{1}{a b} \frac{2}{a b} k D a$

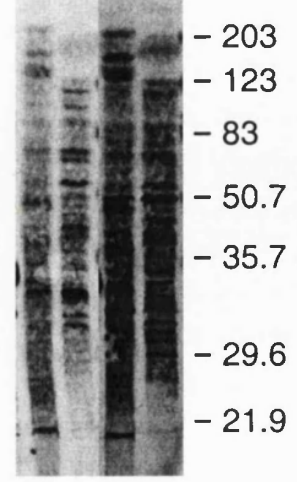

D

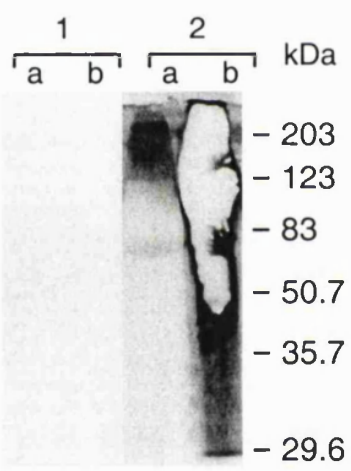

Figure 5.5 Western blot analysis of rabbit $\alpha$-peptide 1 serum (6344) and affinity purification of the serum. (A) TX-100 (a) and SDS soluble schizont proteins (b) separated on a $4-12 \%$ Bis/Tris gel under reducing conditions and stained with Coomassie blue. (B) TX-100 (a) and SDS soluble (b) schizont proteins were transferred by Western blot and incubated with (1) pre-immune and (2) immune serum. (C) BSA (a) and STEVOR peptide 1 conjugated to maleimide activated BSA (b) run on a 10\% SDS/PAGE gel under reducing conditions and stained with Coomassie blue. (D) Western blot analysis of BSA (a) and STEVOR peptide 1 conjugated to maleimide activated BSA (b) incubated with (1) pre-immune and (2) immune serum. Bound antibody was detected by incubation with HRP-conjugated goat anti-rabbit IgG. M=SDS Broad Range marker (Biorad). 
A
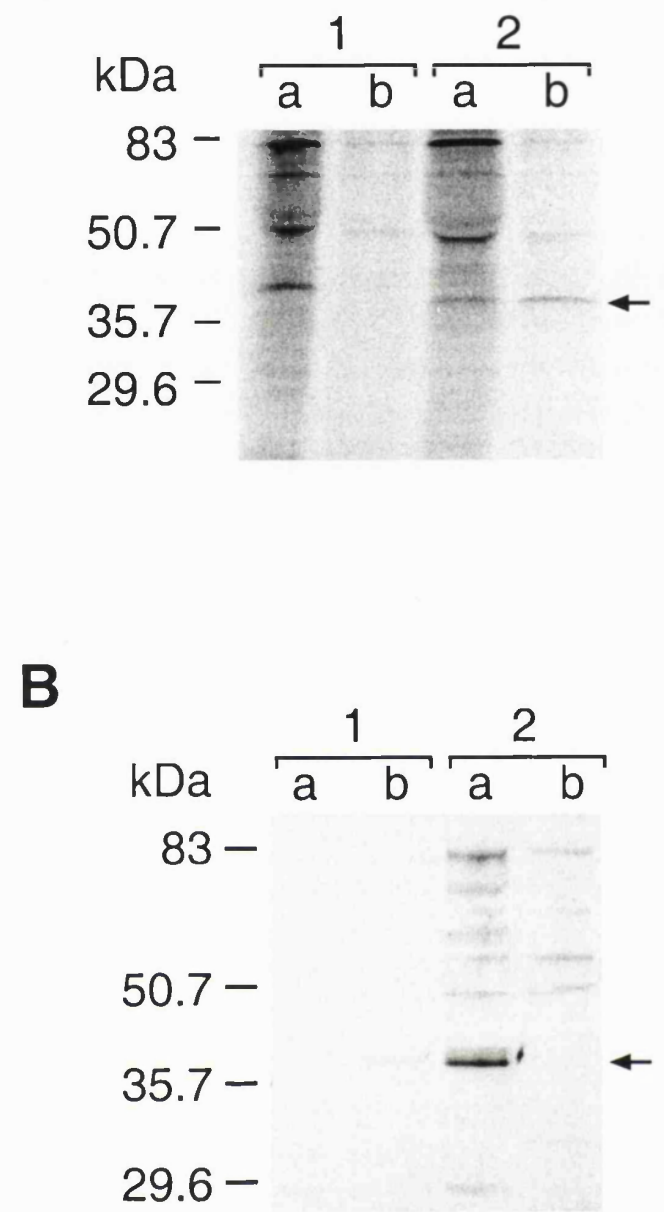

Figure 5.6 Expression of STEVOR in P.falciparum 3D7 parasites. (A) Immunoprecipitation of asynchronous parasite proteins metabolically labelled with $\left[{ }^{35} \mathrm{~S}\right]$ methionine/cysteine and precipitated by rabbit $\alpha$-peptide 1 antibodies (6344). TritonX-100 (a) and SDS (b) soluble protein fractions immunoprecipitated with preimmune (1) and immune (2) serum. The resulting immunoprecipitated proteins were separated on a $12.5 \%$ SDS-PAGE gel under reducing conditions and visualised by fluorography. (B) SDS (a) and TritonX-100 (b) soluble schizont proteins were transferred by Western blot and incubated with mouse anti-peptide 1 pre-immune (1) and immune serum (2). Bound antibody was detected by incubation with HRP- conjugated goat anti-mouse IgG followed by ECL. 
A

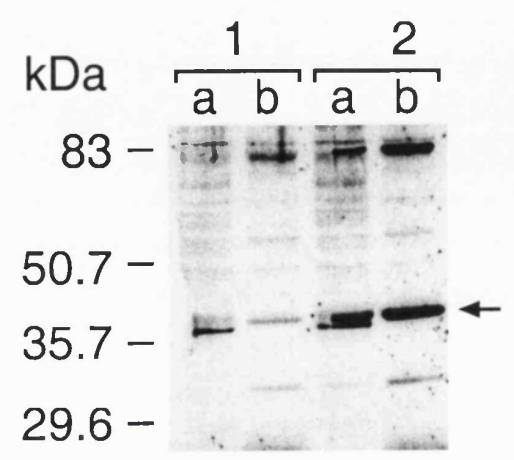

B

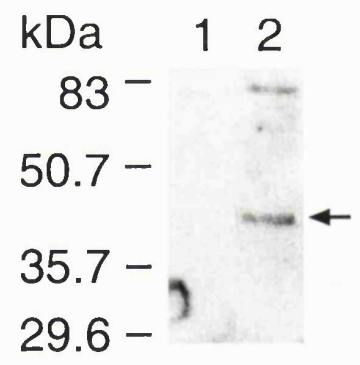

Figure 5.7 (A) Western blot analysis using rabbit $756 \alpha$-peptide 2 antibodies. Schizont protein lysates prepared by either (a) SDS (b) TritonX-100 extraction and separated on a $12.5 \%$ Bis/Tris NuPage gel. The Western blot was probed with (1) preimmune or (2) immune serum against peptide 2. (B) Western blot analysis using mouse $\alpha$-peptide 3 antibodies. Schizont protein lysates prepared by SDS extraction and probed with (1) pre-immune or (2) immune serum against peptide 3. Bound antibody was detected by incubation with horseradish peroxidase (HRP)-conjugated goat anti-rabbit IgG or HRP-conjugated goat anti-mouse IgG followed by and ECL. 
performed to determine the size of the protein recognised by anti-peptide 2 antibodies. This method did not work as the serum failed to immunoprecipitate any proteins.

Anti-peptide 3 antibodies were used to probe a Western blot of TX-100 and SDS extracts of schizont proteins. A band of $\sim 37 \mathrm{kDa}$ in size was detected in the SDS fraction of proteins (Figure 5.7B). Thus serum against peptide 1 and 3 both detected a band of $\sim 37 \mathrm{kDa}$ in the SDS fraction of proteins, indicating that this is the size of STEVOR.

\subsubsection{Antibodies against STEVOR react with fixed 3D7 trophozoites and schizonts}

To identify the location of STEVOR it was first necessary to determine whether any of the anti-STEVOR immune serum reacted with fixed pRBC. An IFA was performed using anti-peptide 1 rabbit serum to analyse binding to fixed thin blood films of 3D7 infected erythrocytes at late trophozoite and schizont stages (Figure 5.8). Parasites were also stained with DAPI (Figure 5.8A) to ensure that $\mathrm{pRBC}$ could be identified by fluorescence. An anti-MSP-1 $\mathrm{mAb}$ that has already been characterised (Blackman et al., 1994) and is known to react with fixed pRBC was used as a positive control for the experiment (Figure 5.8B). The immune anti-peptide 1 serum gave a punctate, cytosolic and membrane-associated fluorescence in schizonts and trophozoites (Figure 5.8D). The IFA pattern observed was different from the pattern observed with the control anti-MSP1 mAb. No staining was observed in the pre-immune rabbit serum denoting that this phenomenon was specific only to the immune serum (Figure 5.8C). Compared with the anti-MSP1 mAb, anti-peptide 1 sera reacted strongly with only $\sim 30 \%$ of pRBC. There appeared to be a weak staining in other pRBC but it was not clear if this staining was specific. Serum raised in mice against peptide 1 were used in an IFA against fixed 3D7 schizonts and they also gave the same punctate pattern as the rabbit serum. Like the rabbit anti-peptide 1 antibodies this serum also reacted strongly with $\sim 30 \%$ of all $\mathrm{pRBC}(5.8 \mathrm{E})$.

To ensure that the antibodies against peptide 1 raised in both mice and rabbits were recognising the same protein a co-localisation experiment using both mouse and rabbit sera against peptide 1was performed. When the two fluorescent images were merged they co-localised suggesting that both sets of antibodies were recognising the protein (data not shown). 

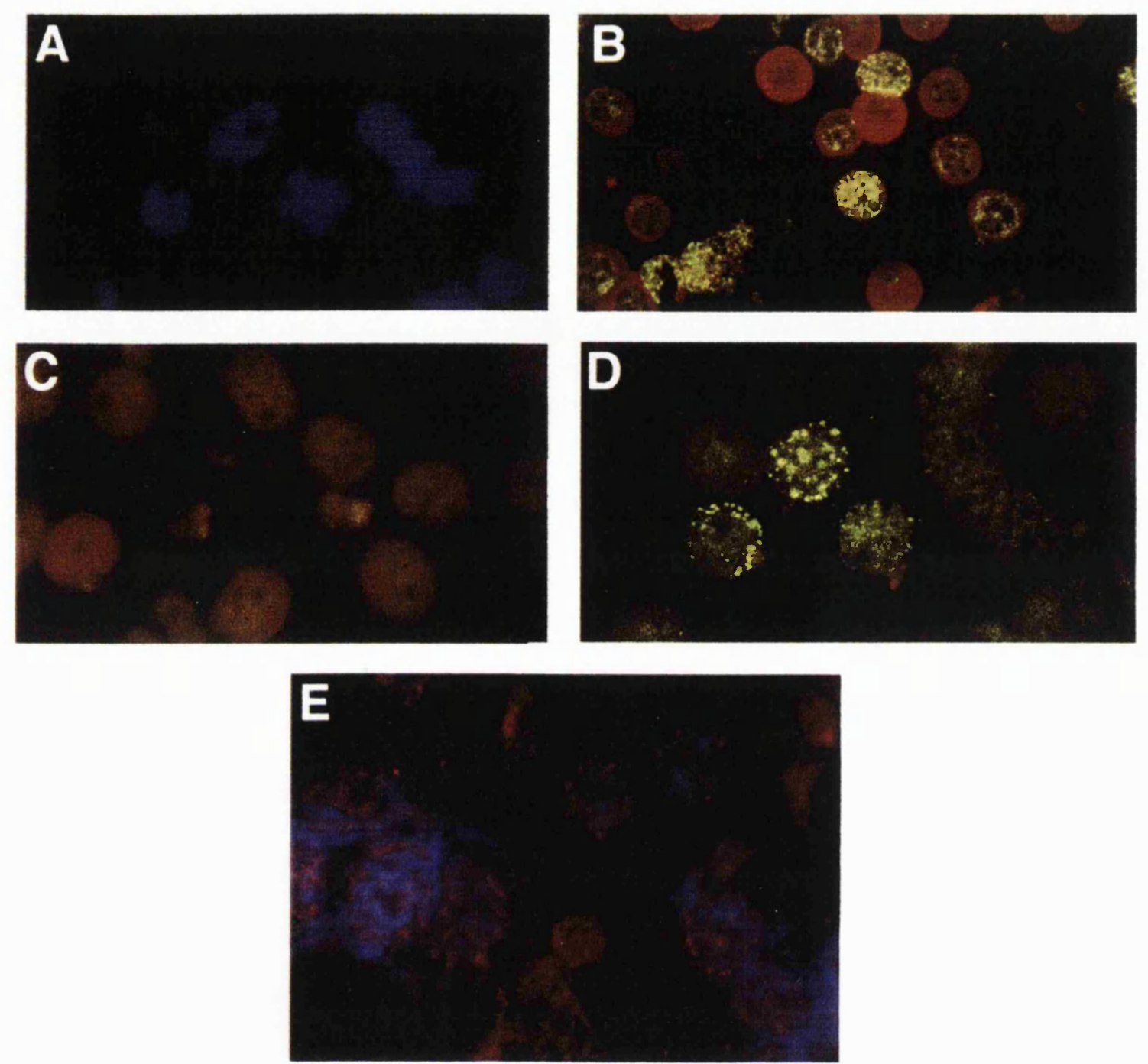

Figure 5.8 $\quad$ IFA of fixed $P$. falciparum 3D7 late stage trophozoites and schizonts. (A) pRBC stained with DAPI (B) mouse $\alpha$-MSP-1 mAb (2F 10) used as a positive control for the IFA (C) rabbit pre-immune serum (6344) used as a negative control for the IFA (D) $\alpha$-peptide 1 rabbit serum (6344). Panel A and D are of the same field. (E) $\alpha$-peptide 1 mouse serum. The secondary antibodies used were FITC-conjugated goat anti-rabbit $\operatorname{IgG}$ (green) and Texas Red-conjugated goat anti-mouse $\operatorname{IgG}$ (red). Smears were viewed under $630 \mathrm{X}$ magnification on a Zeiss fluorescence microscope (Panels A-D) or under $1000 \mathrm{X}$ magnification on an Olympus Delta vision system (Panel E). 
Mouse serum against peptides 2 and 3 gave the same punctate pattern as peptide 1 when used in an IFA against fixed 3D7 schizonts (Figure 5.9) but again only reacted with $\sim 30 \%$ of $\mathrm{pRBC}$.

Serum from both rabbits immunised with peptide 2 exhibited a high reactivity to the peptide as assessed by ELISA (Figure 5.4A). However, it was not possible to obtain a conclusive result on IFA as the pre-immune serum reacted very strongly with $\mathrm{pRBC}$ and no difference could be observed between the pre-immune and immune serum. An attempt was therefore made to remove non-specific antibodies from the pre-immune serum. The pre-immune and immune serum were pre-adsorbed on uninfected RBC lysate in case there were antibodies present which were reactive to human $\mathrm{RBC}$ but on subsequently performing an IFA the background reactivity had still not disappeared (results not shown). Thus, it was impossible to determine the location of STEVOR by IFA using rabbit anti-peptide 2 antibodies.

The IFA staining pattern looked similar using serum raised against all three peptides. A co-localisation IFA was therefore performed with these sera to confirm that they were recognising the same protein. Serum to peptide 1 and peptide 3 were chosen because the induced antibodies recognised peptides located at opposite ends of the STEVOR protein. Figure 5.10 shows that anti-peptide 1 (red) and anti-peptide 3 antibodies (green) give the same punctate fluorescence pattern and appear to co-localise to the same location within the pRBC (Figure 5.10).

It had been observed that P. falciparum parasites maintained in long term culture lose their ability to express knobs. If STEVOR is associated with knobs and it is possible that some of the parasites used in the smears were knob negative, this may be the reason why some cells were not stained with anti-peptide 1 or anti-peptide 3 serum. To test if this was a possibility, knob positive schizonts were selected by gelatin flotation and an IFA experiment performed. Staining of $\mathrm{pRBC}$ with the anti-peptide 1 serum remained low however, staining using $\alpha$-peptide 3 serum increased such that $\sim 90 \%$ of the $\mathrm{pRBC}$ were positive. 


\section{Pep2}

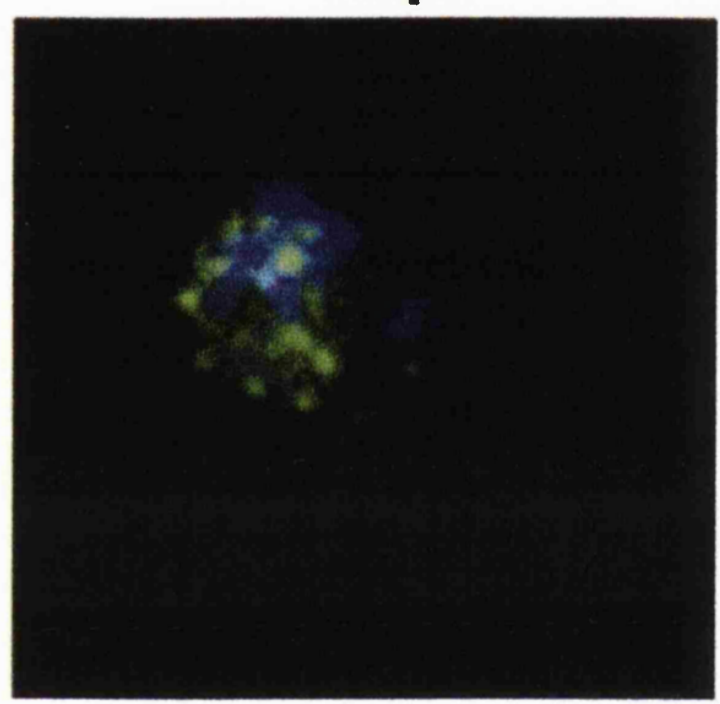

Pep3

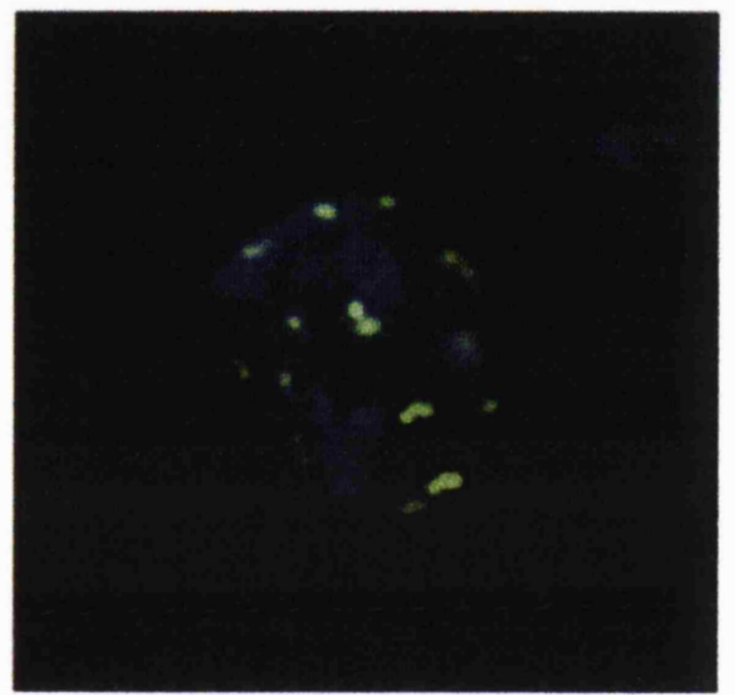

Figure 5.9 IFA of fixed P. falciparum 3D7 late trophozoites and schizonts using mouse serum against peptide (Pep) 2 and Pep 3. The secondary antibody used was FITC-conjugated goat anti-mouse IgG (green). Parasite nuclei were stained with DAPI (blue). The cells were visualised under $1000 \mathrm{X}$ magnification on an Olympus Delta vision system. 


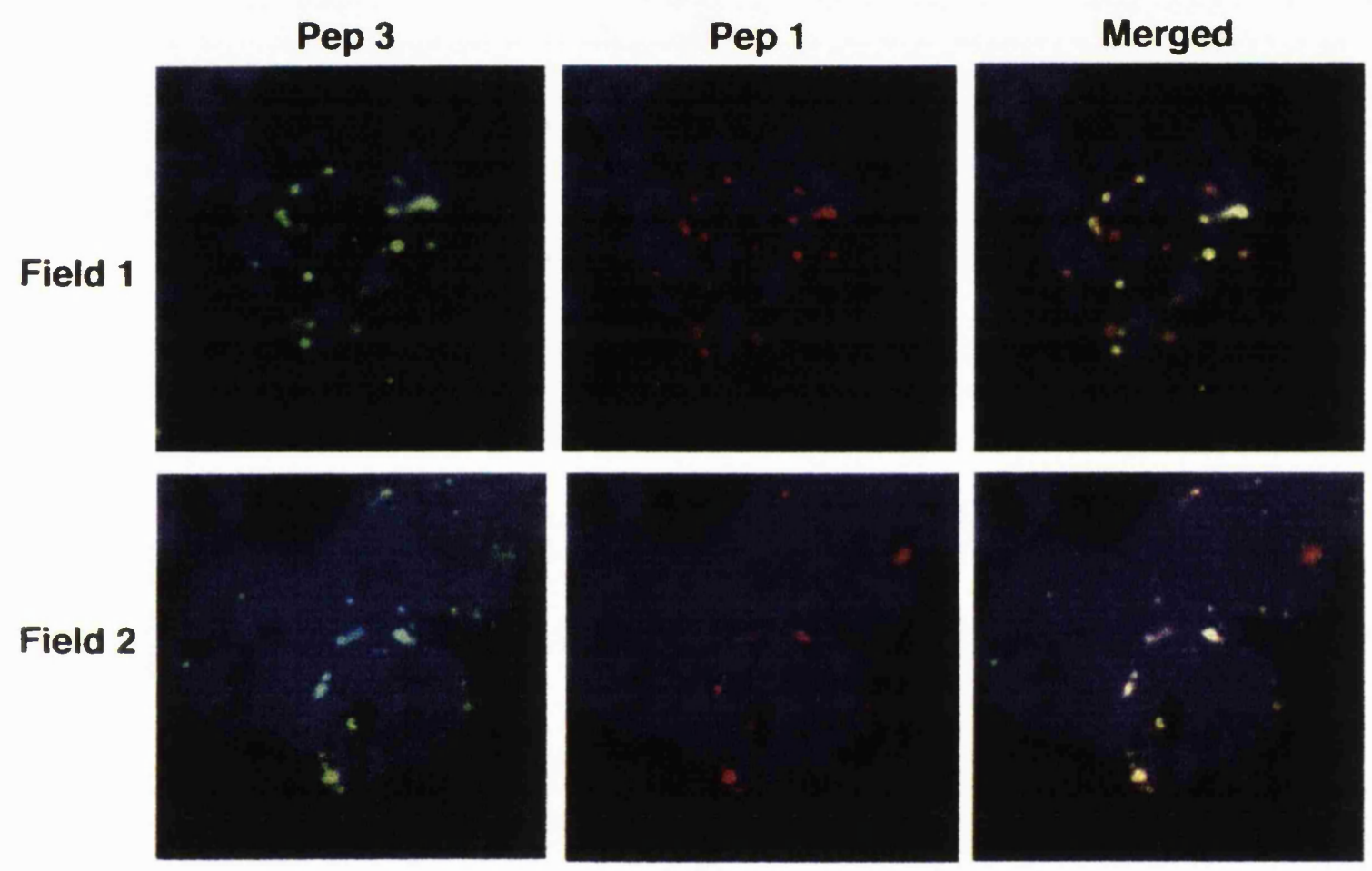

Figure 5.10 Co-localisation IFA of rabbit $\alpha$-peptide 1 and mouse $\alpha$-peptide 3 serum in fixed P. falciparum 3D7 schizonts. Double staining using mouse $\alpha$-peptide 3 (Pep3) serum (green) (Panel 1) and rabbit $\alpha$-peptide 1 (Pepl) serum (red) (Panel 2) and a merging of the two images to demonstrate co-localisation (Panel 3). The secondary antibodies used were FITC-conjugated goat anti-mouse IgG and TRITC-conjugated goat anti-rabbit IgG. Parasite nuclei were stained with DAPI (blue) and cells were visualised under $1000 \mathrm{X}$ magnification on an Olympus Delta vision system. 


\subsubsection{STEVOR is located in the Maurer's Clefts}

IFA studies using only anti-STEVOR antibodies were insufficient to identify the cellular location of STEVOR. pRBC are distorted in the IFA assay and therefore the exact location of antibody binding can only be defined after comparison with antibodies against proteins with known cellular locations. Therefore, a comparison of staining patterns using antibodies against parasite proteins known to be in specific locations within the parasite or pRBC and anti-STEVOR antibodies were performed by dual labelling IFA. As the punctate pattern obtained with the anti-STEVOR serum was reminiscent of rhoptry staining, the first antibody used in the co-localisation studies was against a $52 \mathrm{kDa}$ protein present in the rhoptries (Dr AA Holder, pers comm.), along with the anti-peptide 1 serum raised in rabbits. Analysis of the slides was carried out at 100X magnification using the Olympus Delta Vision imaging system. Rabbit antibodies against peptide 1 clearly did not co-localise with the rhoptry specific $\mathrm{mAb}$ (61.2) (Figure 5.11). Moreover, it is clear that the anti-peptide 1 antibodies reacted with a protein located externally to the rhoptries, possibly within or just underneath the surface of the RBC.

Antibodies against PfSBP-1, a $35 \mathrm{kDa}$ integral protein in the $\mathrm{MC}$, gave a punctate IFA staining pattern very similar to the one seen with the anti-peptide 1 antibody (Blisnick et al., 2000). Double labelling of pRBC using the anti-PfSBP-1 mouse polyclonal sera B28 (Blisnick et al., 2000) and the rabbit anti-peptide 1 serum gave a nearly indistinguishable punctate pattern in mature (schizont) parasites (Figure 5.12). Merging of the two fluorescence patterns showed that STEVOR is associated with the same structures as PfSBP-1. While the punctate pattern observed with anti-PfSBP-1 antibodies can already be observed in relatively young pRBC (late rings/early trophozoites) no evidence of any reactivity with anti-peptide 1 serum is seen in these stages (data not shown).

The first evidence for STEVOR expression is seen in late trophozoite/early schizont stages. Observations in a series of maturing schizonts indicated that the distribution of STEVOR within the parasite changed as the parasite matured (Figure 5.12). In very early schizonts, as indicated by the amount of nuclear material present, the fluorescent punctate pattern appeared to be more diffuse (Figure 5.12A). In these cells only some of STEVOR seems to co-localise with PfSBP-1 (Figure 5.12). In more 
Pep 1

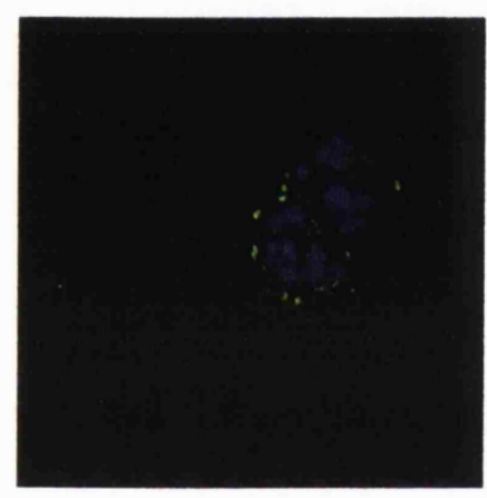

Rhoptry

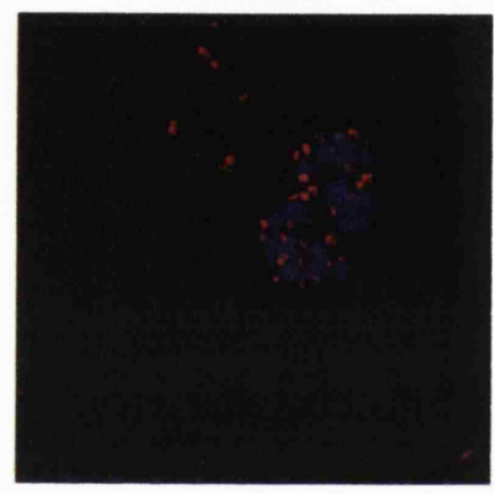

Merged

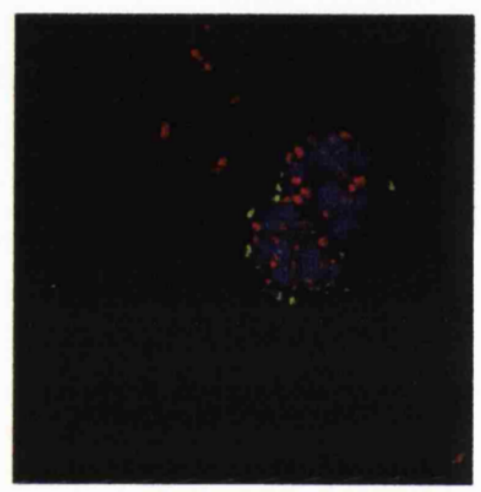

Figure 5.11 Co-localisation IFA with $\alpha$-peptide 1 serum and an $\alpha$-rhoptry antibody in fixed P. falciparum 3D7 schizonts. Double staining using rabbit $\alpha$-peptide 1 (Pepl) serum (green) (Panel l) and a mAb (61.2) against a $52 \mathrm{kDa}$ rhoptry protein (red) (Panel 2) and a merging of the two images (Panel 3). The secondary antibodies used were FITC-conjugated goat anti-rabbit IgG and TR-conjugated goat anti-mouse IgG. Parasite nuclei were stained with DAPI (blue) and the cells were visualised under $1000 \mathrm{X}$ magnification on an Olympus Delta vision system. 
Figure 5.12 Co-localisation IFA with $\alpha$-peptide 1 serum and $\alpha$-PfSBP-1 using fixed P. falciparum 3D7 schizonts. Double staining using rabbit $\alpha$-peptide 1 (Pep1) serum (green) (Panel 1) and mouse serum (B28) against the $77 \mathrm{kDa}$ carboxy terminal of the Maurer's cleft protein PfSBP-1 (red) (Panel 2) and a merging of the two images (Panel 3). The secondary antibodies used were FITC-conjugated goat anti-rabbit IgG and TRgoat anti-mouse IgG. Parasite nuclei were stained with DAPI (blue). Bi-nucleate to multi-nucleate schizonts are shown in rows A-D as determined by staining of nuclear material. The cells were visualised under $1000 \mathrm{X}$ magnification on an Olympus Delta vision system. 
Pep 1

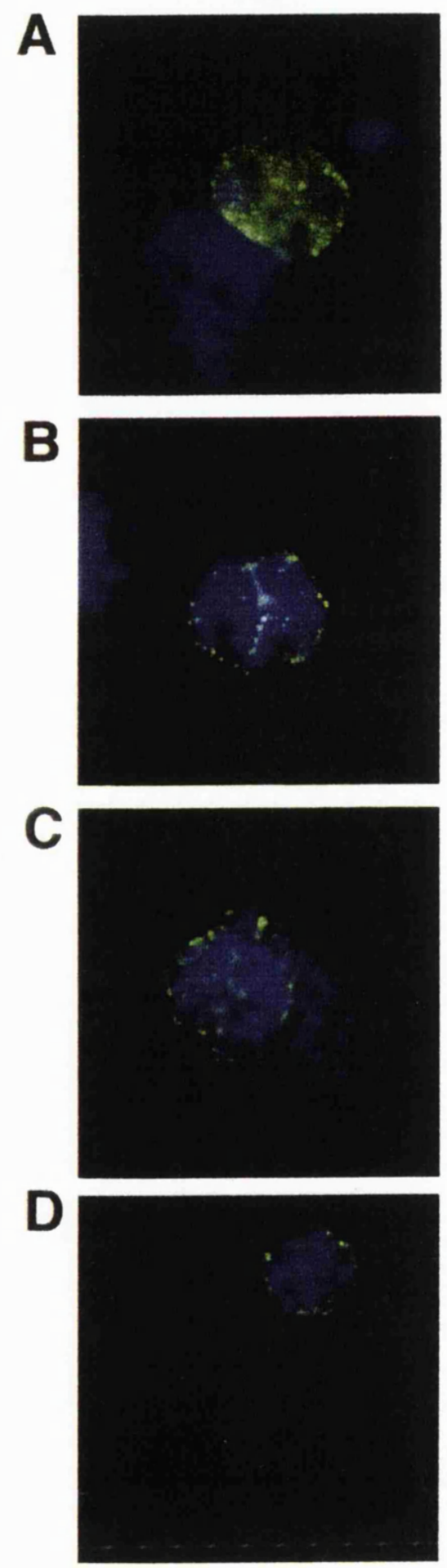

PfSBP-1
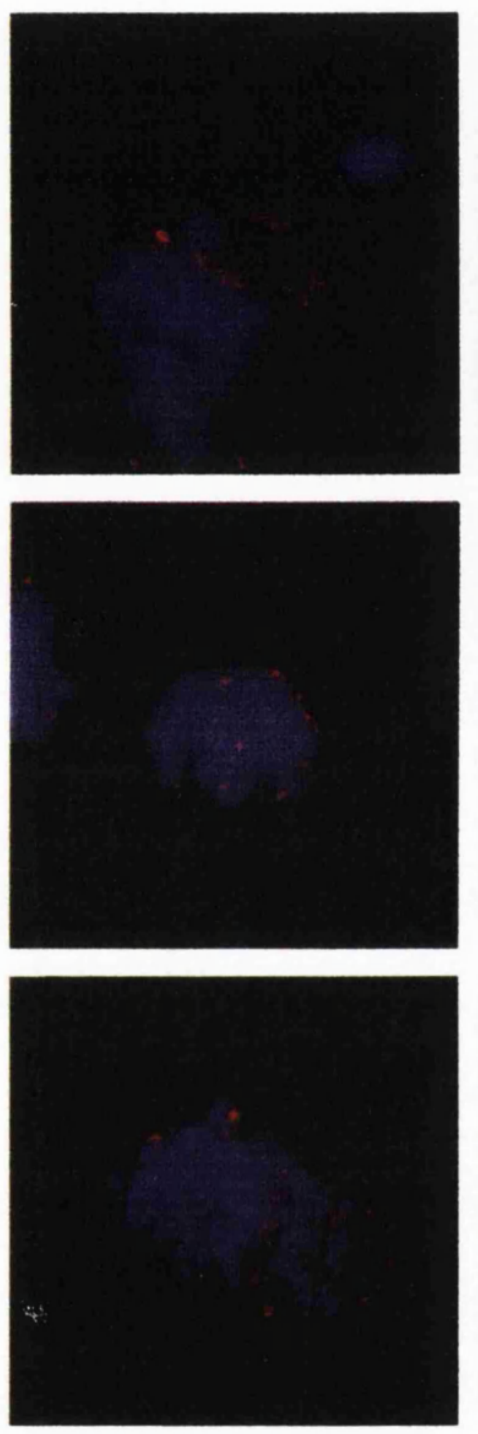

Merged
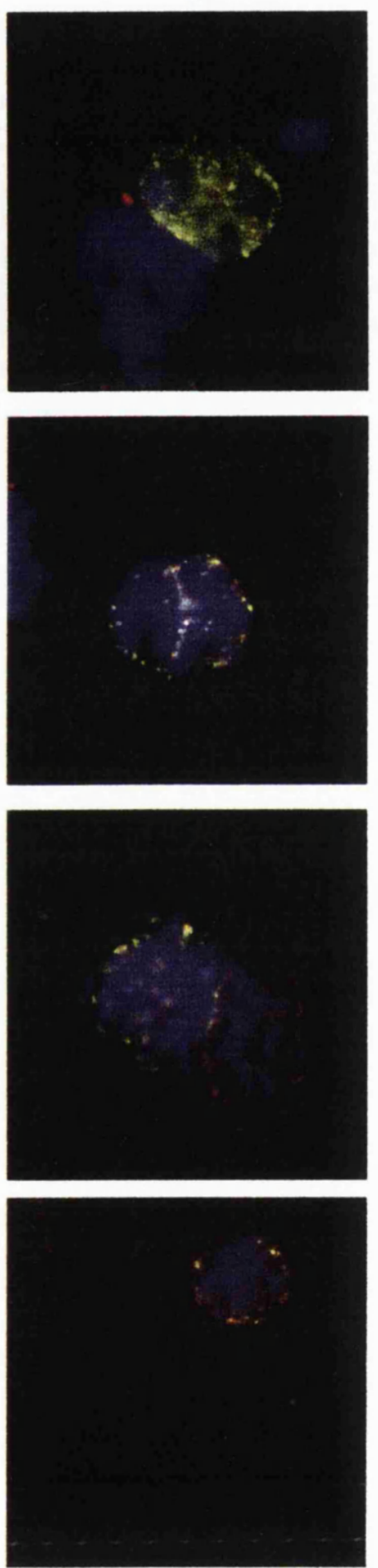
mature schizonts, the punctate pattern became less diffuse (Figure 5.12C \& D) and appeared to redistribute itself in an organised manner within the cell (Figure 5.12B). In fully mature schizonts showing individual staining of each merozoite nucleus, the punctate pattern appeared to be associated peripherally around the $\mathrm{pRBC}$ (Figure 5.12D). This suggests that redistribution of STEVOR, possibly exclusively in MC, occurs by the late schizont stage (Figure 5.12).

Proteins associated with $\mathrm{MC}$, and the structure itself, stay with the $\mathrm{pRBC}$ membrane (termed the RBC ghost), after the schizont has ruptured (Blisnick et al., 2000; Hui and Siddiqui, 1988). To test whether STEVOR is also retained, pRBC ghosts were prepared from late stage schizonts and used for IFA using both anti-peptide 1 and anti-PfSBP-1 antibodies. As can be seen from the DAPI staining no parasite nuclei remain within the pRBC ghost (Fig 5.13A). The typical punctate IFA staining pattern observed in $\mathrm{pRBC}$ is still detected in the pRBC ghost with both anti-peptide 1 as well as anti-PfSBP-1 antibodies (Figure 5.13A) and both antibodies still co-localise to the same structure (Figure 5.13) thereby confirming the location of STEVOR in the MC (Figure 5.13). Antibodies against peptide 3 also stained 3D7 schizont ghosts (Figure 5.13B). However, it was not possible to do a dual staining with anti-peptide 3 and anti-PfSBP-1 antibodies as both sets of antibodies were raised in mice.

PfEMP-3 has been shown to spread around the cytoplasmic surface of pRBC (Waterkeyn et al., 2000). Although the function of this protein is unknown, it has recently been shown that a $3 \mathrm{D} 7$ parasite line in which a truncated form of PfEMP-3 is expressed, the distribution of PfEMP-3 is altered, and it accumulates in structures believed to be MC (Waterkeyn et al., 2000). When an IFA was performed using antibodies against PfEMP-3 and the transfected parasite line a punctate pattern was observed (Waterkeyn et al., 2000). To further confirm that STEVOR is located in the MC, an IFA was performed using anti-PfEMP-3 antibodies (Gruner et al., 2001) and anti-peptide 1 antibodies on schizonts with a truncated PfEMP-3 gene (Figure 5.14). Both antibodies co-localised, resulting in a fluorescent punctate pattern around the pRBC (Figure 15.14). 


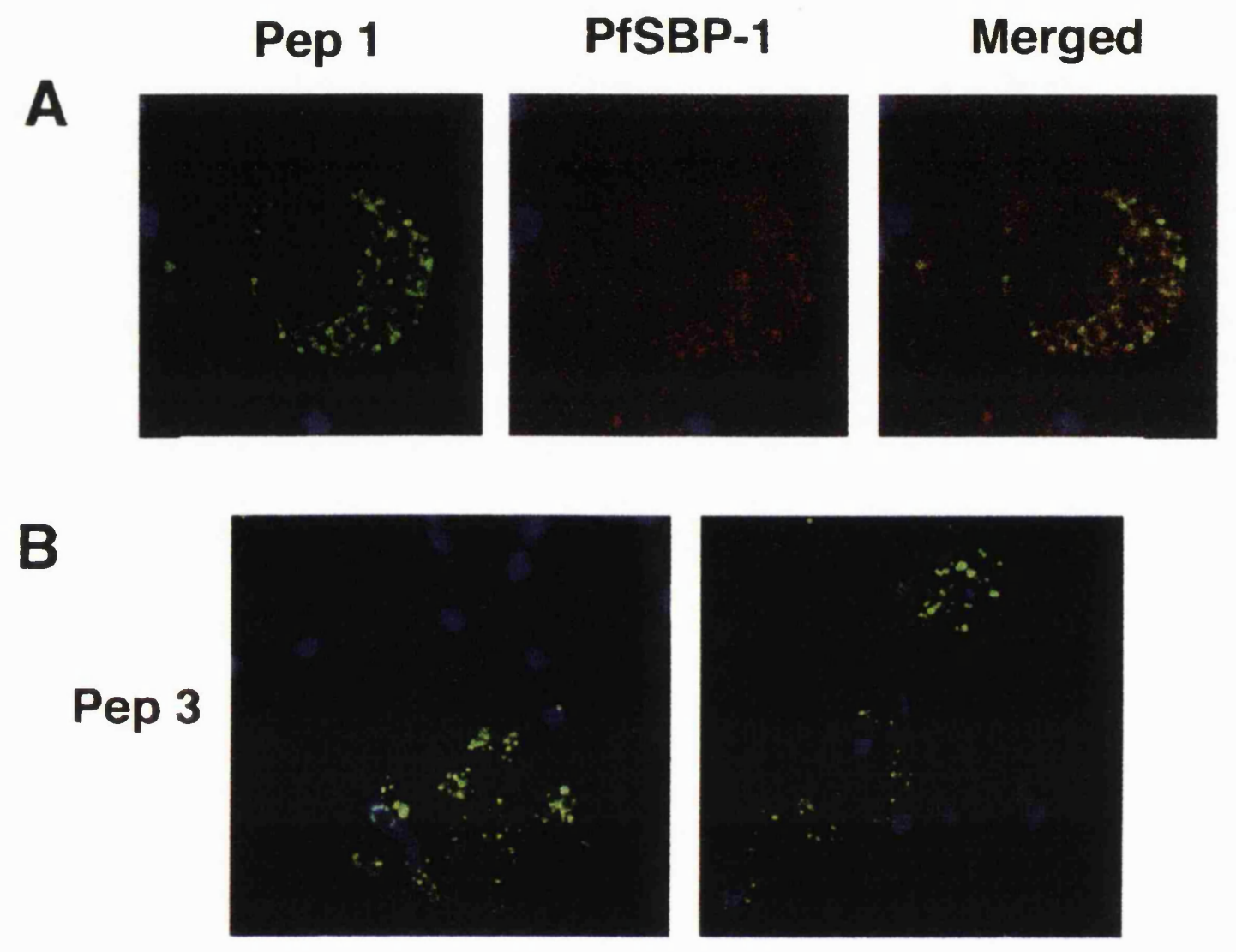

Figure 5.13 IFA using fixed P. falciparum 3D7 schizont ghosts. (A) Co-localisation studies with rabbit $\alpha$-peptide 1 (Pep1) serum (green) (Panel 1) and mouse serum (B28) against the $77 \mathrm{kDa}$ carboxy terminal of the Maurer's cleft protein PfSBP-1 (red)(Panel 2) and a merging of the two images (Panel 3). (B) Mouse $\alpha$-peptide 1 antibodies (green) (Panels $1 \& 2$ showing different fields). The secondary antibodies used were FITCconjugated goat anti-rabbit IgG, FITC-conjugated goat anti-mouse IgG and TR-goat antimouse IgG. Parasite nuclei were stained with DAPI (blue). The cells were visualised under 1000 X magnification on an Olympus Delta vision system. 
Pep 1

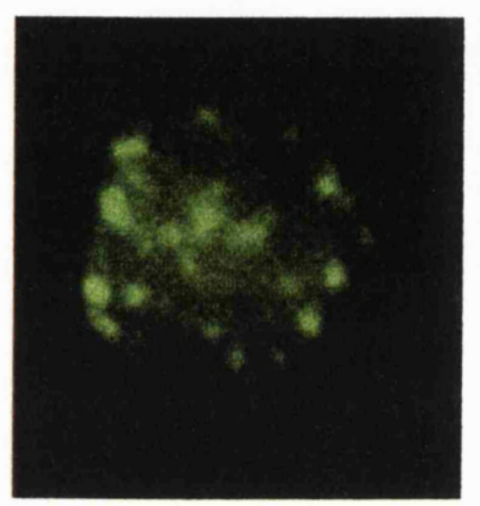

PfEMP-3

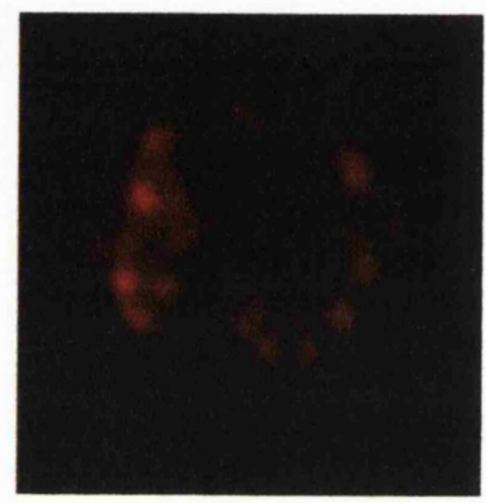

Merged

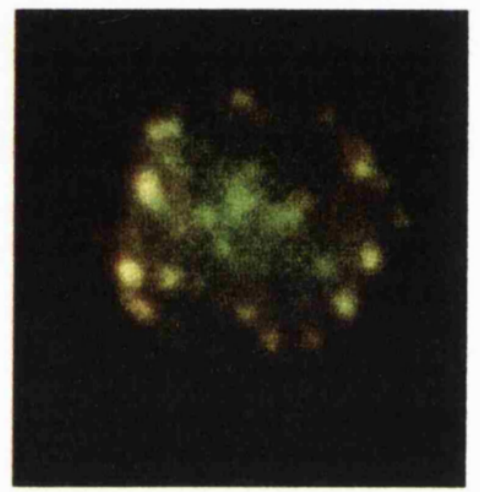

Figure 5.14 IFA using fixed P. falciparum 3D7 PfEMP-3 truncated schizonts. Colocalisation studies with rabbit $\alpha$-peptide 1 (Pepl) serum (green)(Panel 1) and mouse serum (DG662) against the C-terminal of PfEMP-3 (red) (Panel 2) and a merging of the two images to visualise colocalisation (Panel 3). The secondary antibodies used were FITC-conjugated goat anti-rabbit IgG and TR-conjugated goat anti-mouse IgG. Parasite nuclei were stained with DAPI (blue). The cells were visualised under $1000 \mathrm{X}$ magnification on an Olympus Delta vision system. 


\subsubsection{STEVOR is also expressed in gametocytes}

Previous work has shown that stevor is transcribed in gametocytes (Sutherland, 2001). This work has also described alternative splicing of stevor, where different start sites are utilised and hence stevor transcripts from gametocytes encode truncated polypeptides (Sutherland, 2001). These truncated transcripts would encode a protein missing a significant part of the amino terminal end including the region coding for peptide 1 but would contain the peptide 3 region. IFAs were therefore performed using sera against both peptide 1 and 3 on 3D7 gametocytes from culture. No fluorescence was detected with anti-peptide 1 serum (data not shown). However, stage 3-5 gametocytes (Day et al., 1998a) gave a uniform punctate fluorescence pattern on the surface of the gametocyte. In addition, some faint labelling of the whole cell surface with anti-peptide 3 antibodies can also be seen (Figure 5.15). Thus preliminary results suggest that STEVOR is also expressed in gametocytes. It was not possible from this staining to conclusively determine the location of STEVOR within gametocytes.

\subsubsection{Is STEVOR on the surface of pRBC?}

Although our results have demonstrated that STEVOR is located in the MC, protein predictions and its similarity to the RIFINs, suggest that it is also exposed on the pRBC surface. Furthermore, there is evidence to suggest that RBC membrane and surface proteins are translocated via the MC to the RBC membrane (Waterkeyn et al., 2000; Wickham et al., 2001). As all the anti-STEVOR antibodies described above were generated against regions of the protein that were predicted to be cytoplasmic and conserved, it was not possible to determine by the IFA results if STEVOR was exposed on the pRBC surface. Biotin was therefore used to label proteins on the surface of uninfected RBC and pRBC. An SDS extraction of the biotin-labelled cells was made (Figure 5.16) and the proteins were immunoprecipitated using anti-peptide 1 and anti-peptide 3 antibodies. The immunoprecipitated complex was then detected by Western blot using HRP conjugated streptavadin which binds to biotin. As a positive control, the biotin-labelled lysate was also immunoprecipitated with anti-GPA and anti-band 3 antibodies. Band 3 and GPA are present in large amounts on the surface of RBC (Alberts et al., 1994). Unfortunately, no proteins were detected in this assay 
Gam1

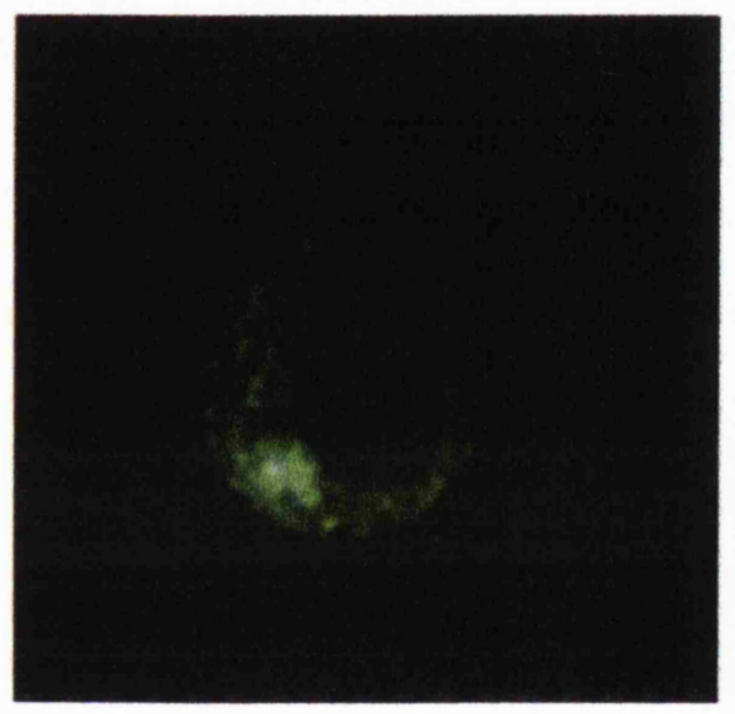

Gam2

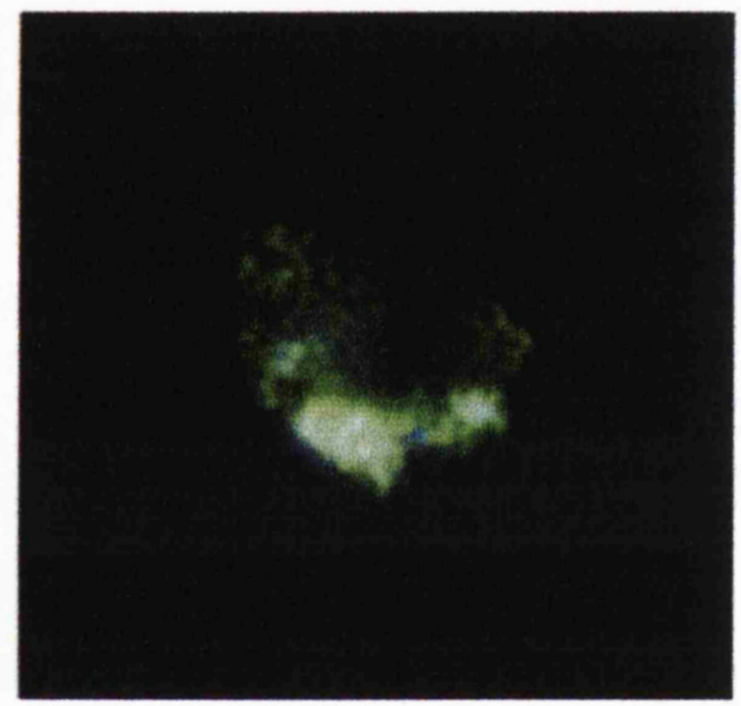

Figure 5.15 IFA using fixed $P$. falciparum 3D7 gametocytes. Fixed gametocytes (Gaml and Gam 2) (stage III-V) were incubated with mouse $\alpha$-peptide 3 serum. Parasite nuclei were stained with DAPI (blue). The secondary antibody used was FITC conjugated goat anti-mouse IgG (green). The cells were visualised under $1000 \mathrm{X}$ magnification on an Olympus Delta Vision system. 


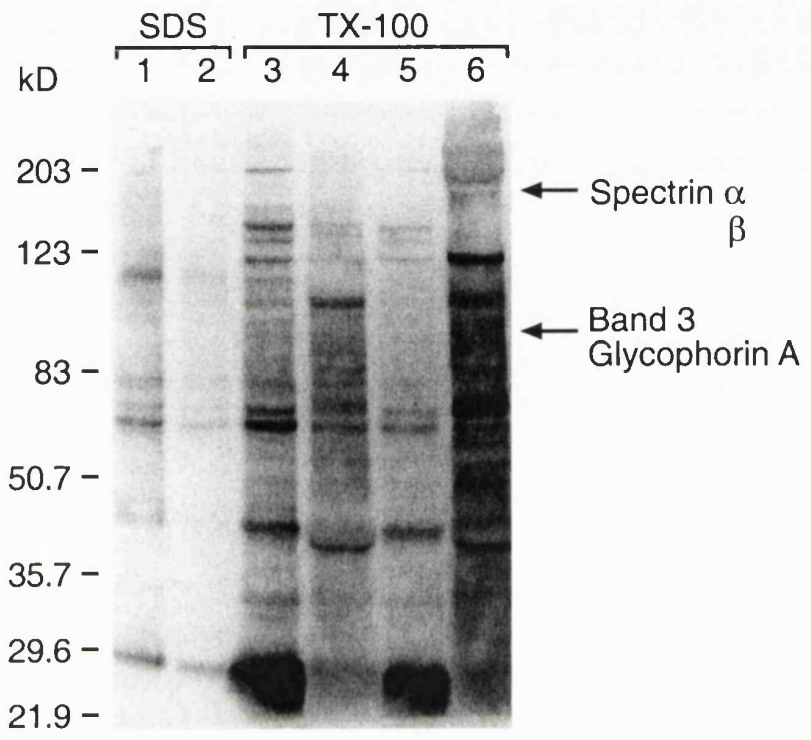

Figure 5.16 Biotinylation of RBC $P$. falciparum 3D7 schizont stage pRBC.

Uninfected RBC (lanes 4 \& 6) or pRBC (lanes 1, 2, 3 \& 5) were incubated with $100 \mu \mathrm{g}(2,5 \& 6)$ or $300 \mu \mathrm{g}(1,3 \& 4)$ of biotin and the proteins extracted by SDS or TritonX-100. Proteins were separated on a $4-12 \%$ Bis/Tris gel and proteins were transferred by Western blot and detected by HRP-conjugated streptavidin followed by ECL. 
including the positive controls (Band 3 and GPA) and therefore it is yet to be determined if STEVOR is exposed on pRBC surface.

\subsubsection{Monoclonal antibodies against STEVOR}

To generate mAbs specific for peptide 1, hybridomas were generated using SP2/0-Ag14 and mouse spleen cells. To determine which mouse spleen to use, the serum after the third boost from the immunised mice was tested by ELISA for reactivity against peptide 1 . The serum was also tested by IFA to make sure it reacted with fixed pRBC. Thus, the mouse whose serum had the highest titre by ELISA and IFA, was chosen, and the spleen used in the fusion. Culture supernatants from the hybridomas were first screened by ELISA to elucidate antibody producing cells specific for the peptide. The polyclonal serum from the mouse used to make the hybridomas served as a positive control. Normal mouse serum was used as the negative control. Hybridomas that were positive by ELISA were then screened by IFA. Wells containing hybridomas that were positive by both ELISA and IFA were expanded in order to reclone. Several of the hybridomas were positive by both tests, but after expansion the culture supernatants were re-tested by IFA and all the hybridomas except one had lost their reactivity by IFA. Only one well remained and this well was expanded and then recloned by limiting dilution. The clones that were positive by ELISA in the $1 \mathrm{cell} / \mathrm{well}$ and 0.1 cell/well dilution series were tested by IFA, but the reactivity had been lost. Thus, the clones that were positive clones with the highest ELISA titre were expanded and re-cloned by limiting dilution again to make sure that they were monoclonal. The culture supernatants from clones in the $0.1 \mathrm{cell} /$ well dilution series were tested by ELISA for specificity for peptide 1 and then expanded. The culture medium was then used to probe TX-100 and SDS extracted proteins on a Western blot. The mAbs 27D2 and 27E11 detected a $\sim 37 \mathrm{kDa}$ protein in the SDS extract (Figure 5.17) similar to that detected by $\alpha$-peptide 1 and $\alpha$-peptide 3 serum indicating that these mAbs were specific for STEVOR. 

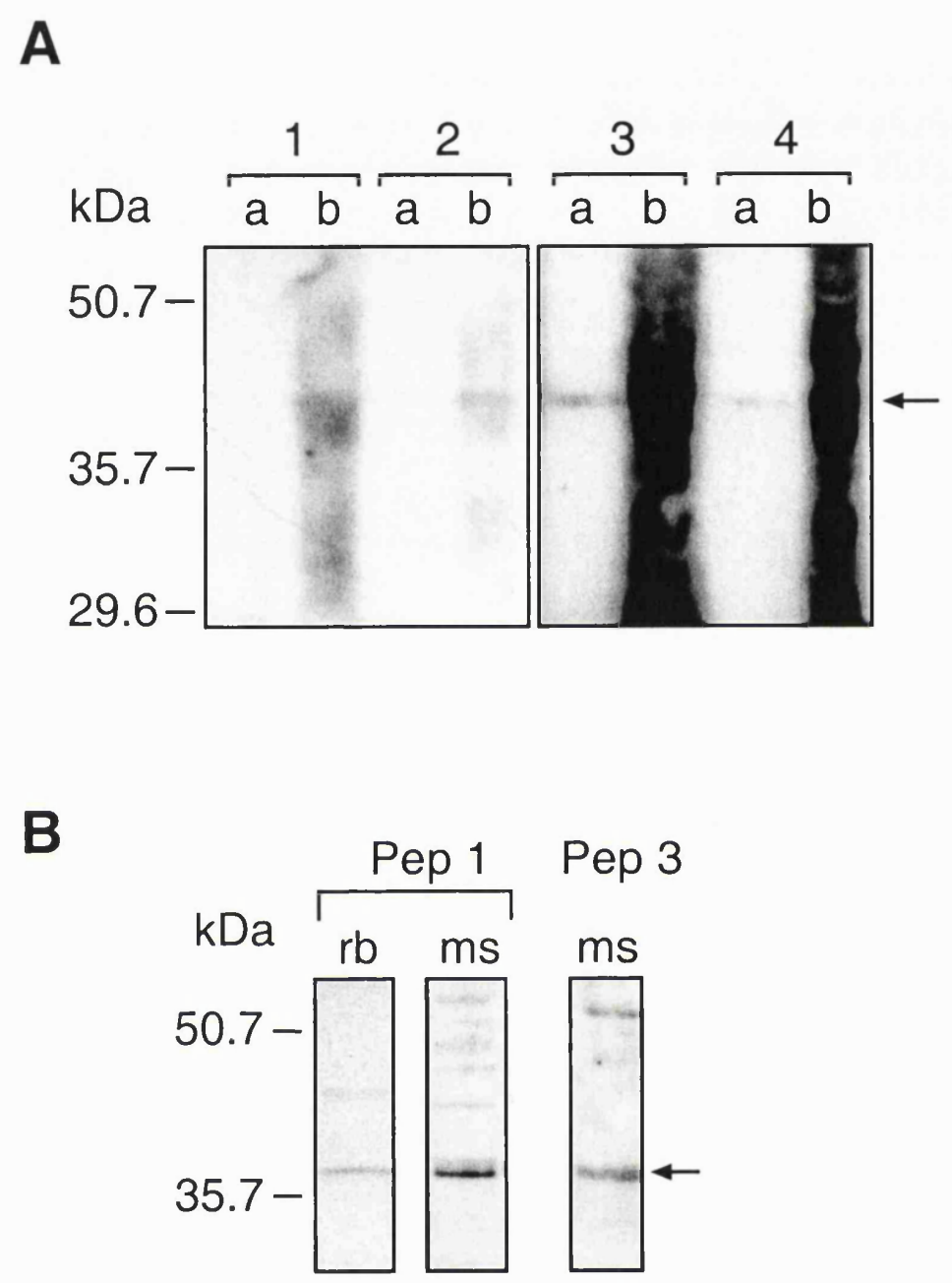

Figure 5.17 Antibodies against STEVOR recognise a protein of $\sim 37 \mathrm{kDa}$. (A) Western using mAb specific for peptide 1. SDS (a) and TritonX-100 (b) soluble schizont proteins separated on a $4-12 \%$ Bis/Tris gel under reducing conditions. The proteins were transferred by Western blot and incubated with 1) normal mouse serum, 2) RPMI complete medium, 3) mAb 27D2, 4) mAb 27E11. Bound antibody was detected by incubation with HRP-conjugated goat anti-mouse IgG followed by ECL. (B) Rabbit (rb) antibodies against peptide 1 precipitated a protein, from an SDS solublised fraction analysed by SDS-PAGE and fluorography, of $\left[{ }^{35} \mathrm{~S}\right]$ methionine/cysteine metabolically labelled asynchronous 3D7 parasites. Mouse (ms) antibodies against peptide 1 and 3 were used to probe an SDS-soluble fraction of schizont extracts analysed by Western blot. 


\subsection{Discussion}

Products of multigene families are very important to protozoan parasites. They perform a number of functional roles, perhaps one of the most important of which is to allow the parasite to establish a chronic infection, as they are antigenically variant. In falciparum malaria, products of the var multigene family also have important virulence characteristics as they play a major role in pathology, principally through sequestration. Therefore, determining the expression and location of proteins encoded by multigene families is essential in understanding their role during parasite survival in the host.

The transcription data obtained in Chapter 4 suggest that STEVOR is expressed in late stage parasites. In order to characterise STEVOR, antibodies were first generated against three peptides. Although antibodies were produced against all the peptides as determined by ELISA, the different peptides were not equally immunogenic. Antibodies against peptides 1 and 3 gave high titres of antibody as demonstrated by ELISA and IFA. However, as only anti-peptide 2 antibodies from one mouse when used undiluted reacted by IFA and ELISA results also indicated low titres of antibody in all mice, it is possible that peptide 2 is not naturally immunogenic. Rabbits immunised with peptide 2 had high titres of antibody, but no clear pattern was distinguishable between the pre-immune and immune serum suggesting that these antibodies were either non-specific or not IFA reactive.

Peptides 1 and 3 are located at opposite ends of the STEVOR protein and sera against these peptides recognised $\mathrm{a} \sim 37 \mathrm{kDa}$ protein in SDS extracts from $\mathrm{pRBC}$. The size and properties of these proteins were consistent with the proposed size (30-40 $\mathrm{kDa})$ and membrane bound characteristic of STEVOR (Cheng et al., 1998). Only one band was immunoprecipitated using $\alpha$-STEVOR antibodies described here, whereas serum generated against the RIFINs, immunoprecipitated more than one band (Kyes et al., 1999). This could be because only a subset of stevor are transcribed and possibly only one expressed, therefore only one band would be expected to be immunoprecipitated.

It was shown by IFA analysis that STEVOR expression starts in late trophozoite stages, consistent with the peak of stevor transcription as described in Chapter 4. In late trophozoites, STEVOR seems to be located in two different areas of the cell, as indicated by a punctate pattern at the periphery of the $\mathrm{pRBC}$ and a more diffuse 
distribution possibly within the PVM or the red cell cytosol. The diffuse distribution disappears rapidly as the parasite matures to schizont stages leaving the more pronounced punctate pattern. The IFA data seems to indicate that STEVOR is accumulating in these defined locations and the diffuse pattern may be indicative of newly expressed STEVOR being translocated to these structures. However, the trafficking of STEVOR has yet to be determined and could be investigated using brefeldin A which has been shown to inhibit malarial proteins trafficking through the MC (Hinterberg et al., 1994). Brefeldin A inhibits protein trafficking via the classical secretory pathway by blocking protein transport vesicles at the ER (Reviewed in Chardin and McCormick, (1999)).

While the punctate pattern observed with the anti-STEVOR antibodies was somewhat reminiscent of IFA location of rhoptry proteins, localisation experiments performed in this study clearly show that STEVOR is located external to rhoptries and the parasite, and is closely associated with the pRBC membrane. There is clear colocalisation of STEVOR with PFSBP-1 a protein associated with MC. MC have been well characterised structurally (Aikawa, 1988) and are membranous structures found at the periphery of $\mathrm{pRBC}$ membranes. Their function has still to be elucidated although they have been implicated in the export of parasite proteins to the RBC membrane (Hui and Siddiqui, 1988). Recent work looking at the transport of the parasite protein KAHRP suggests a possible role of the MC as a pre-assembly and sorting point for P. falciparum proteins on their way to the RBC surface (Wickham et al., 2001). This is consistent with our finding that a truncated form of PfEMP-3 (Waterkeyn et al., 2000) co-localises with STEVOR in the MC. Interestingly, after merozoite release from the pRBC the majority of STEVOR is still found in the remaining red cell ghost with other MC proteins such as PfSBP-1, supporting and strengthening the observation that STEVOR is located in these structures. It is yet to be determined if STEVOR is transported beyond the MC onto the surface of the RBC but their location to these structures may suggest that they are transported to the surface or are involved in some sort of complex with proteins at the surface of the pRBC.

Only $\sim 30 \%$ of cultured pRBC reacted with serum against peptide 1 and 3 , but when only $\mathrm{K}+$ parasites were used for IFA, anti-peptide 3 but not anti-peptide 1 antibodies reacted with nearly all the pRBC. This suggests that STEVOR is only expressed, or expressed to a greater extent, in $\mathrm{K}+$ parasites and therefore, without 
selecting specifically for $\mathrm{K}+$ parasites only a small number of $\mathrm{pRBC}$ are IFA positive. There are several possible explanations for the discrepancy between the sera obtained against peptides 1 and 3. As peptide 1 is not near the carboxy or amino end of the protein and is close to a transmembrane domain, it is possible that antibodies generated against it might be conformationally constrained and may not be accessible in all STEVOR and hence react with only a few pRBC. This could also explain the low levels of staining observed in $\mathrm{pRBC}$ with serum against peptide 1 . Conversely, antibodies generated against peptide 3 would be against the cytoplasmic tail of the protein and would therefore be less conformationally restricted. Alternatively, as alternative splicing of STEVOR products occurs in gametocytes (Sutherland, 2001), it is possible that this also occurs in asexual stages and that only some parasites express the peptide 1 whereas most parasites would express peptide 3 as it is at the carboxy terminus of the protein. P. falciparum does not appear to have conventional signal sequences (Reviewed in Nacer et al., (2001)) and it is also possible that the region encoded for by peptide 1 is a transit peptide that is lost in some forms of the final protein. It would be interesting to elucidate if alternative splicing also occurs in asexual stages and this could be performed by analysing transcription of the whole gene by designing primers that flank the 5' and 3' UTRs.

The expression of STEVOR in late stage parasites and its location in the MC raises a number of questions concerning the possible function of this protein. Most variant antigens described in Plasmodium have mainly been linked to antigenic variation (Handunnetti et al., 1987; Hommel et al., 1983; Howard et al., 1983; Mendis et al., 1988). In contrast, the py235 variant proteins are thought to have a role in host cell receptor/ligand binding during merozoite invasion (reviewed in Khan et al., (2001)). Both PfEMP-1 and RIFIN are exported to the surface of the pRBC relatively early on after invasion. Since my results show that stevor is expressed later than both PfEMP-1 and the RIFINs and it is yet to be determined if it is exposed on the RBC surface it would be expected to contribute some additional/different function for the parasite than PfEMP-1 and the RIFINs.

Some insight into the additional role of STEVOR may come from understanding its role within $\mathrm{MC}$, a part of the $\mathrm{pRBC}$ that is not thought to be directly exposed to host immune responses. Current understanding about the function of $\mathrm{MC}$ suggests a role in regulating proteins trafficking to the surface of the RBC (Hinterberg et al., 1994; 
Waterkeyn et al., 2000; Wickham et al., 2001). Work by Hui and Siddiqui, (1988) has given some indication that components of MC become transiently exposed to the immune system, possibly during the export of parasite proteins to the surface of the $\mathrm{RBC}$ or during internalisation processes of solutes from the surface. While this in itself, would not be expected to elicit a significant immune response, the fact that proteins within the cleft remain with the RBC ghost could lead to strong immune responses against these proteins or proteins complexes. The presence of specific antibodies against components of $\mathrm{MC}$ could then interfere with proper $\mathrm{MC}$ function in the same or subsequent infections. Interestingly, Hui and Siddiqui, (1988) have also shown that accessibility to antibodies against MC proteins only occurs in late stage (schizont) parasites at a time when STEVOR has already migrated to the MC. The presence of STEVOR in the MC could therefore play a dual role by firstly shielding components of the cleft from antibodies during protein export and secondly by hiding cleft proteins in the RBC ghost by shielding them with antigenically variant protein. Winograd et al., (2001) have demonstrated that in vitro, $P$. falciparum pRBC infected membranes stay adhered to C32 melanoma cells after merozoite release and would thus be exposed to the host immune system for long periods of time. Therefore, a protein which is antigenically variant in the Maurer's clefts would shield other proteins associated with the RBC membrane, acting like a "smoke screen", from the host immune system during subsequent infections.

Lysis of the $\mathrm{pRBC}$ to release mature merozoites is a controlled and regulated event potentially involving a range of different parasite proteins (Reviewed in Lew, (2001)). The parasite is in the final stages of development by the time STEVOR reaches the MC. This leaves only lysis of the (three) bounding membranes and the release of merozoites as one of the remaining functions involving proteins associated with the RBC membrane. STEVOR might therefore play a role in ensuring $\mathrm{pRBC}$ lysis and antibodies against STEVOR could disrupt this process.

The important role of STEVOR for parasite function/survival may not just be limited to the erythrocytic cycle. Alternative splicing leading to a truncated RNA has been observed in gametocytes (Sutherland, 2001). The results of the current study with anti-peptide 3, but not anti-peptide 1 antibodies showing a punctate IFA staining of gametocytes similar to that observed in $\mathrm{pRBC}$ is consistent with the expected expression of stevor in gametocytes. It is possible that STEVOR is also located in MC of gametocytes but further work is required, possibly localisation by immunoelectron 
microscopy to distinguish the location in detail. The alternatively spliced transcript would encode a STEVOR protein missing a significant portion of its amino-terminal end but it would still code for the variable region. Therefore, STEVOR could also be located on the surface of the gametocyte. There is evidence in $T$. gondii for a myosin gene which is alternatively spliced at its carboxy-terminal end, with the two different spliced forms MyoB and MyoC having different locations within the cell (Delbac et al., 2001). Furthermore, although PfEMP-1 may be involved in sequestration of early stage (I and II) gametocytes, it is believed that late stage gametocytes utilise a different set of receptors for adhesion (Day et al., 1998b; Rogers et al., 2000). Therefore, if STEVOR locates to the gametocyte surface, it may have a role in sequestration at this stage. Indeed it is more important for the gametocyte to sequester as opposed to asexual stages since it takes the gametocyte around 7 days to mature before it is at the right stage for uptake by the mosquito and is therefore more likely to encounter the host immune response before transmission.

This study has demonstrated that proteins encoded for by a recently characterised multigene family are expressed in both asexual stages and gametocytes. In asexual parasites STEVOR is located in the MC and is expressed later than both PfEMP-1 and the RIFINs. Thus STEVOR may have a role novel from PfEMP-1 which may contribute to the virulence of the parasite and in this context further characterisation of this protein needs to be undertaken. 


\section{CHAPTER SIX}

\section{Final summary and future work}

The malaria parasite is responsible for severe disease world-wide. Understanding parasite-host interactions through gene control is fundamental for the rational design of new drugs and vaccines. The genomic sequencing of several Plasmodium spp. has resulted in the discovery of hundreds of new genes. On closer examination, a number of these genes exist as part of multigene families. Plasmodium species have several multigene families highlighting their importance in parasite survival. Investigations into some members of these families have elucidated their role in parasite immune evasion, virulence and host pathology.

In P. falciparum four multigene families (var, Pf60, rif and stevor) have been currently identified and the sequencing of the 3D7 P. falciparum genome has revealed that they constitute a significant proportion of the genome. In this study, multigene families have been defined as a family of genes comprising of 20 or more members with a similar overall function. The best characterised of these is the var gene family and its products PfEMP-1. Combinations of the roles of these proteins include parasite sequestration, rosetting and antigenic variation, which contribute on the one hand to parasite survival and on the other are intrinsically linked to pathology. Thus, sequestration of $\mathrm{pRBC}$ prevents clearance from the blood in the spleen but conversely sequestration in the fine capillaries of the brain can cause cerebral malaria.

The multigene families in P. falciparum are located next to each other at the telomeric ends of all chromosomes (Bowman et al., 1999). Little is known about how gene expression within Plasmodium multigene families is controlled but their colocalisation sub-telomerically makes it possible that their transcription is linked in a functionally important way. Consequently there is a real need to characterise these genes at a molecular and functional level to elucidate more about parasite virulence and immune evasion.

The work described in this thesis can be divided into two sections and represents a study of a recently defined P. falciparum multigene family, stevor. Thus, the project looked first at the transcription of stevor, and secondly at the expression and localisation of STEVOR in the pRBC. From these results it was possible to speculate on a possible function for STEVOR. 
(1) The genomic and transcriptional analysis of stevor genes. These aims were met by designing stevor specific primers specific for sequences around the region of highest polymorphism. Using these primers in a PCR reaction gave products which, when used to probe a Southern blot of $P$. falciparum 3D7 chromosomes, demonstrated that the primers were universal for most if not all stevor. Sequencing of these PCR products confirmed that they were stevor-derived. Using this data and these reagents it was possible to perform a detailed analysis of stevor transcription. Transcriptional analysis showed that (a) stevor is transcribed during a very tight window of $26-30 \mathrm{~h}$ and (b) that in a parasite population only a subset of the stevor are transcribed although the expression pattern differs between cultures, possibly indicating that different gene subsets are transcribed each time and (c) that even in a single parasite there appears to be multiple transcripts of stevor. To assess if there was a link between var and stevor transcription, parasites were selected on cytoadherence ligands for expression of specific var genes. The work in this thesis does not establish a direct link between the types and chromosomal location of transcriptionally active stevor and the active var locus.

(2) The expression and location of STEVOR proteins. These aims were met by making a number of peptide antibodies specific for different regions of STEVOR. On a Western blot and by immunoprecipitation the antibodies recognised proteins of approximately, $37 \mathrm{kDa}$, the expected size of STEVOR, from 3D7 parasite lysate. Using these antibodies it was established that STEVOR is expressed in late trophozoites and schizonts and not ring stage parasites. Co-localisation studies using a range of different antibodies, locate STEVOR in the MC, a vesicular structure associated with the transport of proteins to the pRBC surface. Upon schizont rupture and merozoite release, STEVOR remains with the pRBC ghost. Furthermore, these antibodies also recognised a protein from gametocytes, indicating that STEVOR is expressed in these stages.

Thus STEVOR is a protein with a variable domain, whose expression is tightly regulated and also not all STEVOR are expressed at one time. It is a protein expressed in both asexual and sexual stages. Stevor transcription does not appear to be linked to var and STEVOR is expressed later than PfEMP-1 and RIFIN during asexual development. This protein is found in structures important for assembly and transport 
of the cytoadherence complex. The findings in this study raise a number of important questions regarding possible functions for this family and its expression product(s) and these lay the foundation for future work which can allow us to elucidate this in more detail.

Is STEVOR exposed on the surface of the cell and if so is it involved in antigenic variation and/ or cytoadherence during any of the stages? STEVOR is encoded by a multigene family and contains a highly polymorphic region predicted to be exposed on the surface of the cell. By analogy to other proteins encoded for by multigene families (PfEMP-1, RIFIN, SICAvar) it could be postulated that STEVOR has a role in antigenic variation. My results suggest that not all stevor are transcribed and therefore possibly only some or one gene is expressed at a given time. It is possible that STEVOR, like PfEMP-1, has a requirement to be antigenically variable and that this differential transcription fulfils this requirement. Furthermore, STEVOR is found in the $\mathrm{MC}$, a structure believed to be important in the assembly and transport to the RBC surface of the cytoadherence complex (Wickham et al., 2001) of which PfEMP-1 is a component. The C-terminal cytoplasmic domain of STEVOR is predicted to be very similar to both PfEMP-1 and RIFIN and it is possible that these proteins are tethered similarly in the red cell membrane forming a multi-protein adhesion complex. From the current study it is clear that gametocytes express STEVOR and gametocytes are also believed to have a different cytoadherence profile to that of trophozoites/schizonts as they sequester in different regions e.g. the bone marrow (Rogers et al., 2000). There is also evidence to suggest that gametocytes bind to different ligands from asexual stages (Day et al., 1998a; Rogers et al., 2000) and therefore STEVOR might mediate sequestration in these stages.

Therefore, it can be argued that although it has not been possible to elucidate in this study, the MC are not the final destination of STEVOR and this protein is exposed on the RBC surface. To determine if STEVOR has a role in antigenic variation similar to those of other multigene families it would first be necessary to establish if STEVOR is ever exposed on the surface of the pRBC. Antibodies could be generated against peptides, as performed in this study, against several of the variable regions, which are believed to be exposed on the surface of the RBC. However, the peptides could only be generated against relatively small regions of the protein and an alternative would be to select larger regions for generation of antibodies by DNA vaccination in mice. The 
variable regions could be selected from the repertoire of genes which this study has shown to be transcribed in culture. IFA staining of live $\mathrm{pRBC}$ using these antibodies could be performed to determine if STEVOR is on the surface. Additionally these antibodies could also be used in an agglutination based assay. Agglutination would only occur if STEVOR was on the surface of the pRBC. Another approach to confirming the location of STEVOR is to look at the transport of STEVOR within the pRBC. My results suggest that the distribution of STEVOR changes as the asexual parasite matures. Transfection studies could be carried out using green fluorescent protein (GFP) as a marker (Wickham et al., 2001) and used to detect STEVOR within the pRBC. Parasites could be transfected with a construct containing a promoter, part of a stevor gene including the predicted signal sequence fused to GFP and a selectable marker. Brefeldin A could be used in this system to determine if STEVOR is transported via the classical pathway. Using a construct to transfect parasites would eliminate the problems of generating several antibodies against stevor to overcome the problem of determining which of the many stevor are expressed. Constructs could be made with differentially spliced forms of STEVOR, and this would enable us to determine if the different forms traffic to the same or different locations in the cell. If the location of these spliced forms is different then it could be possible that STEVOR has multiple roles.

However, the most definitive method for subcellular location would be to perform immunoelectron microscopy. If STEVOR is found on the $\mathrm{pRBC}$, binding assays could be performed. STEVOR could be expressed on COS or CHO cells and then binding assays could be performed to determine if STEVOR binds to any of the known adhesion molecules.

\section{If STEVOR is not located to, and expressed on the pRBC surface then this} poses the interesting question of what possible role(s) could a variant protein play inside the cell? It is possible that STEVOR is not exposed on the RBC surface but like other proteins encoded by multigene families $(P y 235, P f 60)$ it may play a role inside the cell. Since STEVOR is expressed late during parasite development, it is possible that it is involved in schizont rupture or merozoite release. Alternatively, as it is located in the $\mathrm{MC}$ it may have a role of interacting with other proteins in the $\mathrm{MC}$ or at the RBC membrane during transport or interacting with other proteins at the cytoadherence complex. It has been described that the $\mathrm{MC}$ become transiently exposed in late parasite 
stages (Hui and Siddiqui, 1988). Parasites were incubated in vitro with a mAb against a MC protein which is not exposed on the pRBC surface. Subsequent immune electron microscopy of the parasites localised the mAb primarily on membranes of $\mathrm{MC}$ within the infected erythrocyte's cytosol (Hui and Siddiqui, 1988). Therefore, to address the above questions, parasites in culture could be incubated with antibodies against STEVOR. This would determine if STEVOR is important during the late developmental stages of the parasite, (i.e. schizogony) or whether it plays a role in schizont rupture and/or merozoite release. Alternatively, parasite development may only be affected during the next erythrocytic cycle. An IFA after incubation with antiSTEVOR antibodies would deduce if antibodies made against STEVOR are internalised. This would determine whether the internal components of the parasite are somehow still exposed to the host immune system during development. It would be important for the parasite to protect a structure such as the MC, which may be critical for protein transport, with STEVOR acting like a smoke screen with respect to the host immune response. Finally, these questions can possibly be addressed by "knocking out" all of STEVOR by using anti-sense RNA to the conserved regions of the gene

What is the role of STEVOR in natural infections in man? It would initially be of importance to determine if the in vivo transcription profile seen in field isolates derived directly from natural infections reflects that observed in culture, i.e., whether only a subset of stevor genes are transcribed, by analysing transcription in field isolates. Cheng et al., (1998) have already shown that very different stevor repertoires exist, even between cloned parasite lines. If the extent of diversity of stevor in field isolates is similar to var, it may implicate a role in antigenic variation. It would also be of great importance to determine if STEVOR was immunogenic in natural infections. This may suggest a protective role for antibodies against STEVOR during infection and ELISA based assays could be used to do this. The relatively conserved regions of STEVOR could be expressed and serum from individuals in malaria endemic countries used in an ELISA to determine if there are antibody responses against these regions of the protein. Alternatively, polymorphic regions of genes transcribed in field isolates could be expressed and used in this type of assay. Another assay could be used to determine whether people who are exposed to malaria make a memory response ( $\mathrm{B}$ or $\mathrm{T}$ cell) against STEVOR. Leukocytes could be obtained from the blood of malaria exposed individuals and incubated with STEVOR protein. Proliferation of certain subsets of 
lymphocytes would indicate a memory response against STEVOR, which could be analysed by thymidine incorporation or by the addition of a fluorescent dye that is halved upon cell division. The amount of dye can be detected by flow cytometry and this approach would also allow the analysis of which subset(s) of lymphocytes is/are responding.

Finally, it is possible to use the several databases available to search for functional STEVOR homologues in other Plasmodium spp or other pathogens in order to elucidate a possible role for this protein. Homologues of stevor in a rodent or simian Plasmodium spp. would also allow one to perform assays to look at STEVOR functions in vivo. However, searches to date have not yet found any homologues of STEVOR.

In conclusion, multigene families have already been defined as being important for protozoan parasites. It is crucial that more work on STEVOR is carried out to further understand parasite survival and parasite induced host pathology. A variant protein now known to be expressed in two stages and in structures important in protein trafficking may have important implications for parasite development as well as evasion of the host immune response. The work carried out in this study has laid the foundations for the study of stevor, a multigene family with as yet undefined but possibly novel roles in malaria biology. 


\section{APPENDIX 1}

\section{Medium, Solutions and Buffers}

(All solutions were made up in $\mathrm{dH}_{2} \mathrm{O}$ unless otherwise stated)

\section{Coomassie Stain}

45\%(v/v) Methanol

$10 \%(\mathrm{v} / \mathrm{v}) \quad$ Ethanoic acid

$0.1 \%(\mathrm{v} / \mathrm{v}) \quad$ Coomassie Brilliant Blue R

\section{Complete medium}

RPMI. comp (Gibco BRL)

$10 \% \quad \mathrm{v} / \mathrm{v}$ foetal calf serum

L-glutamine $\quad 0.2 \mathrm{mM}$

$1 \% \mathrm{v} / \mathrm{v}$ antibiotics $\quad 100 \mathrm{U} / \mathrm{mL}$ penicillin and $100 \mu \mathrm{g} / \mathrm{mL}$ streptomycin

\section{Diethanolamine buffer}

$\begin{array}{ll}4.85 \%(\mathrm{v} / \mathrm{v}) & \text { diethanolamine } \mathrm{pH} 9.8 \\ 0.2 \mathrm{M} & \mathrm{MgCl}_{2} 6 \mathrm{H}_{2} \mathrm{O} \\ 0.1 \% & \mathrm{NaN}_{3}\end{array}$

\section{Destain}

$5 \%(\mathrm{v} / \mathrm{v}) \quad$ Methanol

$10 \%(\mathrm{v} / \mathrm{v}) \quad$ Acetic acid

\section{Extraction Buffer for Isolation of Genomic DNA \\ $50 \mathrm{mM} \quad$ Tris $\mathrm{HCl} \mathrm{pH7.5}$ \\ $50 \mathrm{mM} \quad$ EDTA \\ $100 \mathrm{mM} \quad \mathrm{NaCl}$}

\section{Gel Buffer (Lower)}

$1.5 \mathrm{M} \quad$ Tris Base

$0.4 \% \mathrm{w} / \mathrm{v} \quad \mathrm{SDS}($ final $\mathrm{pH}=8.8$ )

Gel Buffer (Upper)

$0.5 \mathrm{M} \quad$ Tris Base

$0.4 \% \mathrm{w} / \mathrm{v} \quad \mathrm{SDS}$ (final $\mathrm{pH}=6.8$ )

Giemsa Staining buffer (10X)

$0.2 \mathrm{M}$ disodium hydrogen orthophosphate

$0.044 \mathrm{M}$ potassium dihydrogen orthophosphate (final $\mathrm{pH}=7.2$ )

\section{Hybridisation Solution}

$0.36 \mathrm{M} \quad \mathrm{NaCl}$

$20 \mathrm{mM} \quad$ Sodium phosphate buffer $\mathrm{pH} 7.7$

$\left(\mathrm{NaH}_{2}(1 \mathrm{M}) 4.2 \mathrm{~mL}, \mathrm{Na}_{2} \mathrm{H}(1 \mathrm{M}) 36.2 \mathrm{~mL}\right.$ in final volume $\left.200 \mathrm{~mL}\right)$

$2 \mathrm{mM} \quad$ EDTA

$1 \% \mathrm{v} / \mathrm{v} \quad$ SDS 
$0.5 \% \mathrm{w} / \mathrm{v} \quad$ Skimmed milk Powder

$10 \% \mathrm{v} / \mathrm{v} \quad$ Dextran Sulphate

$0.25 \mathrm{mg} / \mathrm{mL}$ Denatured salmon Sperm DNA

\section{Hypotonic lysis buffer}

$5 \mathrm{mM} \quad$ Tris base

$5 \mathrm{mM} \quad$ Sodium azide

$5 \mathrm{mM} \quad$ Magnesium chloride

$1 \mathrm{mM} \quad$ dithiothreitol

$1 \mathrm{mM} \quad$ EGTA

$\mathrm{pH} 7.5$

\section{Immunoprecipitation Wash buffer I}

50 mMTris- $\mathrm{HCl} \mathrm{pH} 8.2$

$5 \mathrm{mM} \quad$ EDTA

$0.5 \% \mathrm{v} / \mathrm{v} \quad \mathrm{TX}-100$

$1 \mathrm{mg} / \mathrm{mL} \quad$ BSA

$0.5 \mathrm{M} \quad \mathrm{NaCl}$

\section{Immunoprecipitation Wash buffer II}

$50 \mathrm{mM} \quad$ Tris-HCl pH 8.2

$5 \mathrm{mM} \quad$ EDTA

$0.5 \% \mathrm{v} / \mathrm{v} \quad \mathrm{TX}-100$

\section{Krebs Buffered Saline (KBS)}

$\begin{array}{ll}6.670 \mathrm{~g} / \mathrm{L} & \mathrm{NaCl} \\ 0.341 \mathrm{~g} / \mathrm{L} & \mathrm{KCl} \\ 0.284 \mathrm{~g} / \mathrm{L} & \mathrm{MgSO}_{4} \\ 222.2 \mathrm{~mL} & \text { Buffer* }\end{array}$

${ }^{*} \mathrm{pH}$ adjusted to $\mathrm{pH} 7.4$ by addition of the above volume of the following buffer

$\begin{array}{ll}3.094 \mathrm{~g} & \mathrm{Na}_{2} \mathrm{HPO}_{4} \\ 4.360 \mathrm{~g} & \mathrm{NH}_{4} \mathrm{Cl} \\ 217.86 \mathrm{ml} & \mathrm{dH}_{2} \mathrm{O}\end{array}$

\section{LB Agar}

15g/L Agar

in LB Broth

LB Broth

$10 \mathrm{~g} / \mathrm{L} \quad \mathrm{NaCl}$

$10 \mathrm{~g} / \mathrm{L} \quad$ bacto-tryptone

$5 \mathrm{~g} / \mathrm{L} \quad$ yeast extract

\section{Loading Buffer(6x)}
$0.25 \%$
bromophenol blue
$40 \%$
w/v sucrose 
Parasite Lysis buffer:

$0.5 \mathrm{M}$ EDTA pH7.5

$0.1 \mathrm{M}$ Tris, $1 \% \mathrm{w} / \mathrm{v}$ Sarkosyl

$1 \mathrm{mg} / \mathrm{mL}$ Proteinase $\mathrm{K}$

Parasite culture medium:

RPMI 1640 medium

$5 \mathrm{~g} / \mathrm{L}$

Albumax

$0.2 \mathrm{mM}$

L-glutamine

Phosphate Buffered Saline (PBS)

$0.1 \mathrm{M}$

$\mathrm{NaCl}$

$80 \mathrm{mM}$

$\mathrm{Na}_{2} \mathrm{HPO}_{4}$

$20 \mathrm{mM}$

$\mathrm{pH}=7.5$

$\mathrm{NaH}_{2} \mathrm{PO}_{4}$

SDS Extraction Buffer

$1 \% \mathrm{w} / \mathrm{v} \quad$ SDS

$5 \mathrm{mM} \quad$ EDTA

$50 \mathrm{mM} \quad$ Tris- $\mathrm{HCl} \mathrm{pH8.8}$

SDS-PAGE 10\% Homogenous Acrylamide Gel (to make 5 gels)

$8 \mathrm{~mL} \quad$ Lower gel buffer

$10.7 \mathrm{~mL} \quad 30 \%$ acrylamide (Sigma)

$200 \mu \mathrm{L} \quad 10 \%$ persulphate (Sigma)

$30 \mu \mathrm{L} \quad$ TEMED (Sigma)

13.3mL $\quad \mathrm{dH}_{2} \mathrm{O}$

SDS-PAGE Running Buffer (5X)

$\begin{array}{ll}0.025 \mathrm{M} & \text { Tris base } \\ 0.192 \mathrm{M} & \text { Glycine } \\ 0.1 \% \mathrm{w} / \mathrm{v} & \text { SDS }\end{array}$

SDS-PAGE Stacker Gel Mixture (to make 5 gels)

$2.5 \mathrm{~mL} \quad$ Upper gel buffer

$1.58 \mathrm{~mL} \quad 30 \%$ acrylamide

$\quad 40 \mu \mathrm{L} \quad 10 \%$ persulphate

$13 \mu \mathrm{L} \quad$ TEMED

$5.92 \mathrm{~mL} \quad \mathrm{dH}_{2} \mathrm{O}$

SDS Sample Buffer (2X)

$0.125 \mathrm{M} \quad$ Tris base

$20 \% \mathrm{w} / \mathrm{v} \quad$ Glycerol

$4.5 \% \mathrm{w} / \mathrm{v} \quad$ SDS

$0.01 \% \mathrm{w} / \mathrm{v} \quad$ Bromophenol blue 


\section{SSPE Buffer (1X)}

$120 \mathrm{mM}$

$\mathrm{NaCl}$

$15 \mathrm{mM}$

tri-NA citrate

$13 \mathrm{mM}$

$\mathrm{KH}_{2} \mathrm{PO}_{4}$

$1 \mathrm{mM}$

EDTA

Tris borate EDTA Buffer (1X)

$10.8 \mathrm{~g} / \mathrm{L}$

Tris

$22 \mathrm{~g} / \mathrm{L}$

Boric acid

$0.75 \mathrm{~g} / \mathrm{L}$

EDTA

\section{Tris EDTA (TE) Buffer (1X)}

$10 \mathrm{mM}$

Tris

$1 \mathrm{mM}$

EDTA

Triton-X 100 extraction buffer

$1 \% \mathrm{v} / \mathrm{v} \quad$ Triton $\mathrm{X}-100$

$5 \mathrm{mM} \quad$ EGTA and EDTA

$150 \mathrm{mM} \quad \mathrm{NaCl}$

$50 \mathrm{mM} \quad$ Tris- $\mathrm{HCl} \mathrm{pH} 8.0$

\section{Western Transfer Buffer}

$3 \mathrm{~g} / \mathrm{L}$

Trizma base

$14.5 \mathrm{~g} / \mathrm{L} \quad$ Glycine

$200 \mathrm{~mL} \quad$ Methanol 


\section{APPENDIX 2}

ClustalX alignment of predicted STEVOR sequences obtained from the http://plasmodb.org/PlasmoDB.shtml site. The regions corresponding to STEVOR specific peptides are described. Peptide 1 (black bar), Peptide 2 (red bar) and Peptide 3 (blue bar) were designed to conserved regions of the STEVOR protein. 
chrblab 004446
chr 2 11953 a

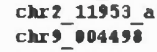
${ }_{\operatorname{chr} 12}^{\operatorname{ch} 16} 16 \mathrm{C}$ chr $12-16$ a chr11-11.321336,1 Chrs $100 \overline{4} 472$ Chrbīab B84 287 part Chri $1 \overline{2}-16$ b chr1 100063 chro-012227 Chr2 11953 b
chrblab_004435-b
chrblab_002778 chr4 000586

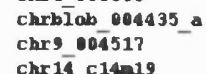
Chr14_c14a19
chr11_c110549 chr 11953 c $c$.

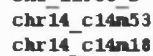
chr11-c1191308 Chr 13-02083 clustal Consensus

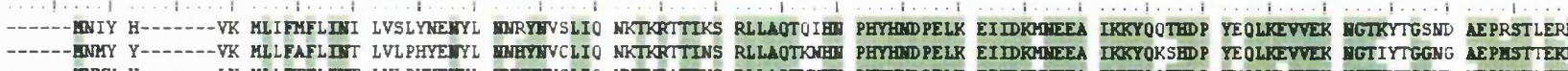
TWYY Y

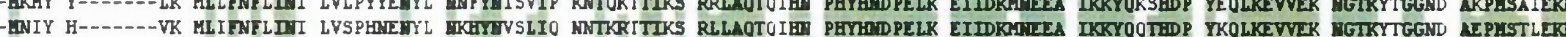

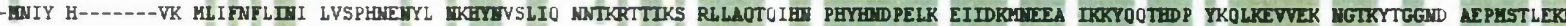
-

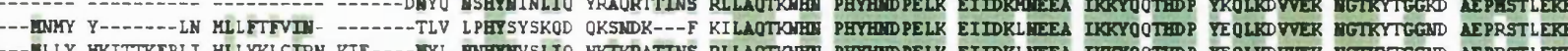

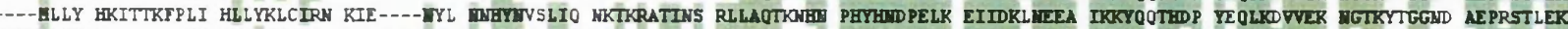

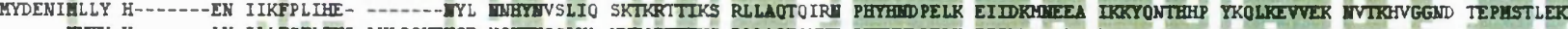

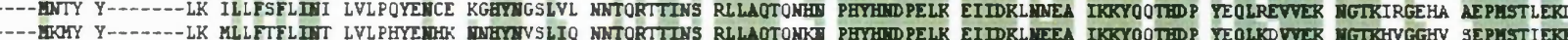

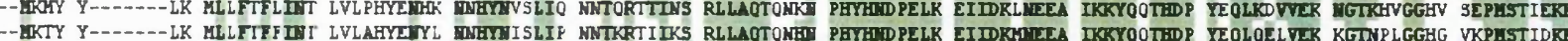

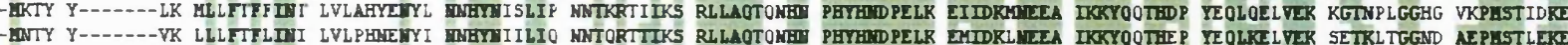

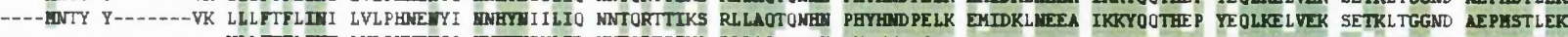

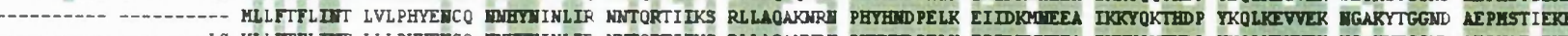
D.
D.

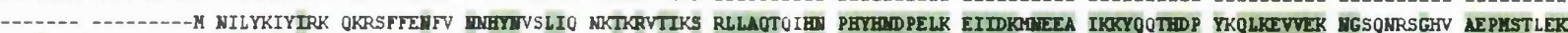

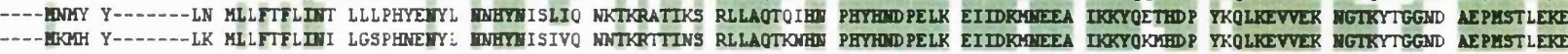

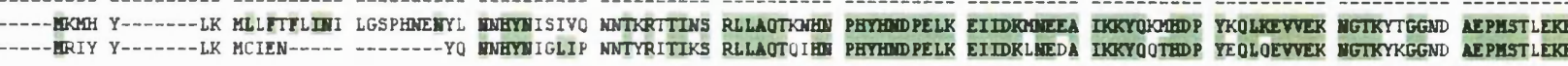

chrolow_004446 chrs 211953 a chr $1 \overline{2}-16$ c chr12-16-a carbiób. 004254 Clurg_004472 chrblotb-004 287 chr 12 16 b chr1 100623 Chr' 0.02227 Chrr 211953 b chrblob $002770^{-}$ chrt_.00586 chrbīob_-004435_a chr 14 c14nes chr11 c11m54, Ch2_11953_c chr11_c14n53 Chr 14 C14an 18

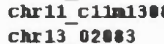
chrblob_0026 Cluetal Consenes

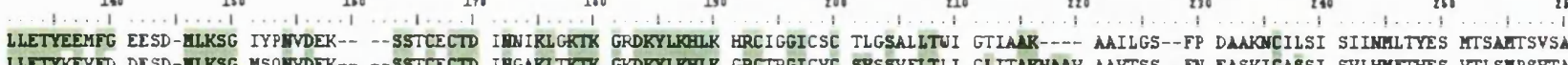

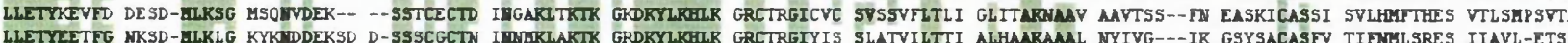

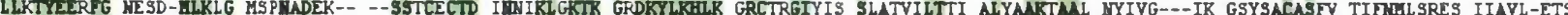

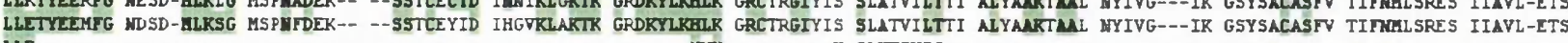

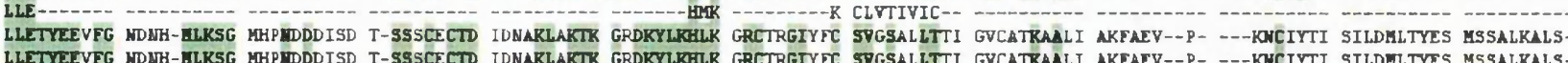

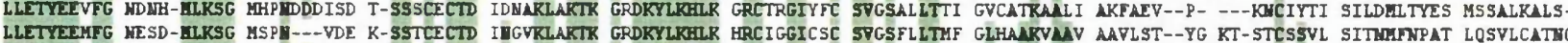
LUETYELMFG DEC-HIILKSG KYOHVDE--- K-SSTCECTD IIGGKLSKTK GRDKYLKKLR HRCIGGICSC STGSFLLTMF GLHAARAML AEF---TKYG TTYSACKSSI TTYSHLSSDS ----BIAGS LLETYYEMFG DEC-HELLRSG KYOMVDE--- K-SSTCECTD IMGGKLSKTK GDDKYLKKLLR HRCIGGICSC SYGSFLLTHF GLHAARAML AEF---TKYG TTYSACKSSI TTYSHLSSDS ----KTAGST

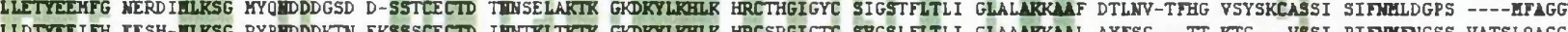
U

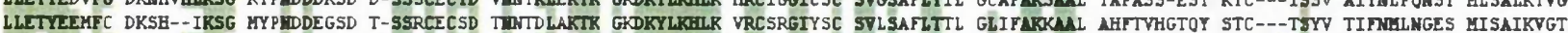

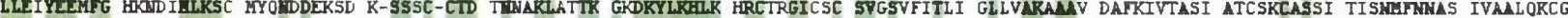

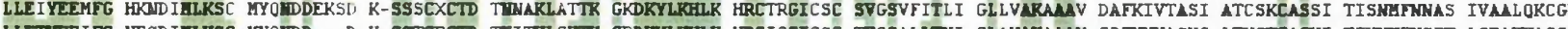

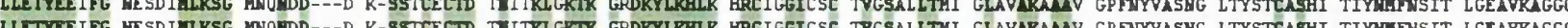

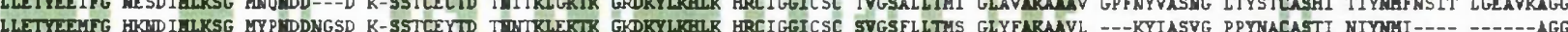

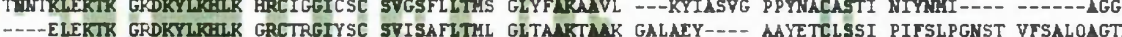

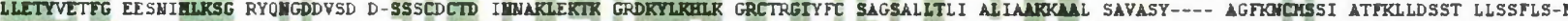

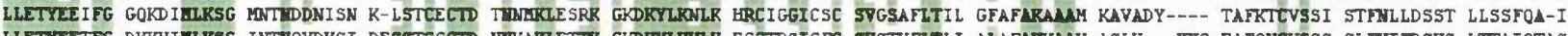

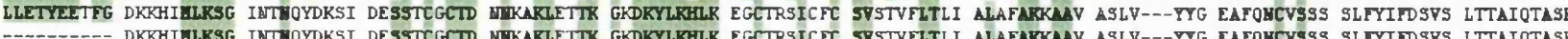

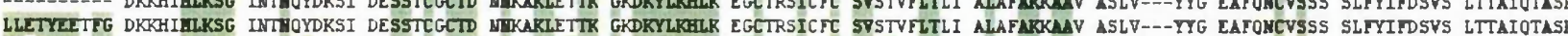

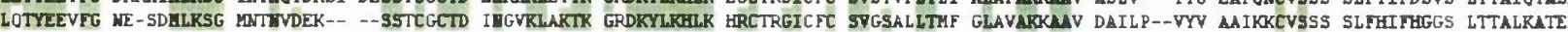




\begin{tabular}{|c|c|c|c|c|c|c|c|}
\hline & & & & & & & \\
\hline & & & & VLLILAVVLI & ILYIULYRRR & KASYKBIECKKK & BuCR \\
\hline & ASG & & & VLLILTVULI & ILYIJLYRRR & & \\
\hline 1 & & & & & & & \\
\hline & GFCASC & & & & ILYTL: & KISUKB & \\
\hline & - IOACASDL & & & & & & \\
\hline chr9_00-04472 & & & & & & & \\
\hline chrbīab_004287part & --CTIGASDV & & & & & & \\
\hline chr9_00-4566 & ACFT---DL & & & & & & \\
\hline $\operatorname{chr} 1 \overline{2}-1$ & ACFT---DL & & & & & & HLCR \\
\hline chris 100 & $A C S A---D L$ & TGNAA & & & & & \\
\hline chr $9-0022$ & TCAT-GISDI & AGTVS & & & & & HeCK \\
\hline chr2_11953 & TCAN-GA & AGTVS & & & & & \\
\hline chrb & VCAT- & & & & & & $\mathrm{AlCR}$ \\
\hline chrb & & & & & & & \\
\hline chr 4 & & & & & & & \\
\hline 135_a & & & & & & & \\
\hline & & & & & & & \\
\hline & & & & & & & \\
\hline & & & & & & & \\
\hline & & & & & & & \\
\hline chr & & & & & & & \\
\hline chr & & & & & & & \\
\hline & & & & & & & HLCR \\
\hline chr & & & & & & & BLCR \\
\hline - & SYAGP-DT & VIPATGAM & AFPPYGI & PLLILAV & ILYULLYRR & & alck \\
\hline
\end{tabular}




\section{REFERENCES}

Adams, J. H., Sim, B. K., Dolan, S. A., Fang, X., Kaslow, D. C., and Miller, L. H. (1992). A family of erythrocyte binding proteins of malaria parasites, Proc Natl Acad Sci U S A 89, 7085-9.

Aikawa, M. (1988). Morphological changes in erythrocytes induced by malarial parasites, Biol Cell 64, 173-81.

Aikawa, M., Uni, Y., Andrutis, A. T., and Howard, R. J. (1986). Membraneassociated electron-dense material of the asexual stages of Plasmodium falciparum: evidence for movement from the intracellular parasite to the erythrocyte membrane, Am J Trop Med Hyg 35, 30-6.

Albano, F. R., Berman, A., La Greca, N., Hibbs, A. R., Wickham, M., Foley, M., and Tilley, L. (1999a). A homologue of Sar1p localises to a novel trafficking pathway in malaria-infected erythrocytes, Eur J Cell Biol 78, 453-62.

Albano, F. R., Foley, M., and Tilley, L. (1999b). Export of parasite proteins to the erythrocyte cytoplasm: secretory machinery and traffic signals, Novartis Found Symp 226, 157-72; discussion 173-5.

Alberts, B., Bray, D., Lewis, J., Raff, M., Roberts, K., and Watson, J. (1994).

Molecular Biology of the Cell (New York, Garland Publishing, Inc.).

al-Khedery, B., Barnwell, J. W., and Galinski, M. R. (1999). Antigenic variation in malaria: a 3' genomic alteration associated with the expression of a P. knowlesi variant antigen, Molecular Cell 3, 131-41.

Allison, A. (1954). Protection afforded by sickle-cell trait against subtertian malarial infection, British Medical Journal 1, 290-294.

Allred, D. R., Carlton, J. M., Satcher, R. L., Long, J. A., Brown, W. C., Patterson, P. E., O'Connor, R. M., and Stroup, S. E. (2000). The ves multigene family of B. bovis 
encodes components of rapid antigenic variation at the infected erythrocyte surface, Molecular Cell 5, 153-62.

Allred, D. R., Cinque, R. M., Lane, T. J., and Ahrens, K. P. (1994). Antigenic variation of parasite-derived antigens on the surface of Babesia bovis-infected erythrocytes, Infect Immun 62, 91-8.

Altin, J. G., and Pagler, E. B. (1995). A one-step procedure for biotinylation and chemical cross-linking of lymphocyte surface and intracellular membrane-associated molecules, Anal Biochem 224, 382-9.

Anders, R. F., and Saul, A. (2000). Malaria vaccines. [Review] [23 refs], Parasitology Today 16, 444-7.

Atkinson, C. T., and Aikawa, M. (1990). Ultrastructure of malaria-infected erythrocytes, Blood Cells 16, 351-68.

Ballou, W. R., Hoffman, S. L., Sherwood, J. A., Hollingdale, M. R., Neva, F. A., Hockmeyer, W. T., Gordon, D. M., Schneider, I., Wirtz, R. A., Young, J. F., and et al. (1987). Safety and efficacy of a recombinant DNA Plasmodium falciparum sporozoite vaccine, Lancet 1, 1277-81.

Barnwell, J. W. (1990). Vesicle-mediated transport of membrane and proteins in malaria-infected erythrocytes, Blood Cells 16, 379-95.

Barnwell, J. W., Howard, R. J., Coon, H. G., and Miller, L. H. (1983a). Splenic requirement for antigenic variation and expression of the variant antigen on the erythrocyte membrane in cloned Plasmodium knowlesi malaria, Infect Immun 40, 985-94.

Barnwell, J. W., Howard, R. J., and Miller, L. H. (1983b). Influence of the spleen on the expression of surface antigens on parasitized erythrocytes, Ciba Found Symp 94, 117-36 
Barry, J. D., Graham, S. V., Fotheringham, M., Graham, V. S., Kobryn, K., and Wymer, B. (1998). VSG gene control and infectivity strategy of metacyclic stage Trypanosoma brucei, Mol Biochem Parasitol 91, 93-105.

Baruch, D. I., Ma, X. C., Singh, H. B., Bi, X., Pasloske, B. L., and Howard, R. J. (1997). Identification of a region of PfEMP1 that mediates adherence of Plasmodium falciparum infected erythrocytes to CD36: conserved function with variant sequence, Blood 90, 3766-75.

Baruch, D. I., Pasloske, B. L., Singh, H. B., Bi, X., Ma, X. C., Feldman, M., Taraschi, T. F., and Howard, R. J. (1995). Cloning the P. falciparum gene encoding PfEMP1, a malarial variant antigen and adherence receptor on the surface of parasitized human erythrocytes [see comments], Cell 82, 77-87.

Bate, C. A., Taverne, J., and Playfair, J. H. (1989). Soluble malarial antigens are toxic and induce the production of tumour necrosis factor in vivo, Immunology 66, $600-5$.

Benting, J., Mattei, D., and Lingelbach, K. (1994). Brefeldin A inhibits transport of the glycophorin-binding protein from Plasmodium falciparum into the host erythrocyte, Biochem $J$ 300, 821-6.

Berendt, A. R. (1993). Sequestration and its discontents: infected erythrocyteendothelial cell interactions in Plasmodium falciparum malaria, Res Immunol 144, 740-5; discussion 754-62.

Berendt, A. R., Simmons, D. L., Tansey, J., Newbold, C. I., and Marsh, K. (1989). Intercellular adhesion molecule- 1 is an endothelial cell adhesion receptor for Plasmodium falciparum, Nature 341, 57-9.

Biggs, B. A., Anders, R. F., Dillon, H. E., Davern, K. M., Martin, M., Petersen, C., and Brown, G. V. (1992). Adherence of infected erythrocytes to venular endothelium selects for antigenic variants of Plasmodium falciparum, J Immunol 149, 2047-54. 
Bischoff, E., Guillotte, M., Mercereau-Puijalon, O., and Bonnefoy, S. (2000). A member of the Plasmodium falciparum Pf60 multigene family codes for a nuclear protein expressed by readthrough of an internal stop codon, Mol Microbiol 35, 100516.

Blackman, M. J., Dennis, E. D., Hirst, E. M., Kocken, C. H., Scott-Finnigan, T. J., and Thomas, A. W. (1996). Plasmodium knowlesi: secondary processing of the malaria merozoite surface protein-1, Exp Parasitol 83, 229-39.

Blackman, M. J., Scott-Finnigan, T. J., Shai, S., and Holder, A. A. (1994). Antibodies inhibit the protease-mediated processing of a malaria merozoite surface protein, J Exp Med 180, 389-93.

Blisnick, T., Morales Betoulle, M. E., Barale, J. C., Uzureau, P., Berry, L., Desroses, S., Fujioka, H., Mattei, D., and Braun Breton, C. (2000). Pfsbp1, a Maurer's cleft Plasmodium falciparum protein, is associated with the erythrocyte skeleton, $\mathrm{Mol}$ Biochem Parasitol 111, 107-21.

Bonnefoy, S., Bischoff, E., Guillotte, M., and Mercereau-Puijalon, O. (1997). Evidence for distinct prototype sequences within the Plasmodium falciparum Pf60 multigene family, Molecular \& Biochemical Parasitology 87, 1-11.

Borre, M. B., Owen, C. A., Keen, J. K., Sinha, K. A., and Holder, A. A. (1995). Multiple genes code for high-molecular-mass rhoptry proteins of Plasmodium yoelii, Mol Biochem Parasitol 70, 149-55.

Borst, P., and Ulbert, S. (2001). Control of VSG gene expression sites, Mol Biochem Parasitol 114, 17-27.

Bowman, S., Lawson, D., Basham, D., Brown, D., Chillingworth, T., Churcher, C. M., Craig, A., Davies, R. M., Devlin, K., Feltwell, T., et al. (1999). The complete nucleotide sequence of chromosome 3 of Plasmodium falciparum, Nature 400, 5328. 
Bradley-Moore, A. M., Greenwood, B. M., Bradley, A. K., Bartlett, A., Bidwell, D. E., Voller, A., Craske, J., Kirkwood, B. R., and Gilles, H. M. (1985). Malaria chemoprophylaxis with chloroquine in young Nigerian children. II. Effect on the immune response to vaccination, Ann Trop Med Parasitol 79, 563-73.

Brown, K. N., and Brown, I. N. (1965). Immunity to malaria: antigenic variation in chronic infections of Plasmodium knowlesi, Nature 208, 1286-8.

Brown, K. N., and Hills, L. A. (1974). Antigenic variation and immunity to Plasmodium knowlesi: antibodies which induce antigenic variation and antibodies which destroy parasites, Trans R Soc Trop Med Hyg 68, 139-42.

Buffet, P. A., Gamain, B., Scheidig, C., Baruch, D., Smith, J. D., Hernandez-Rivas, R., Pouvelle, B., Oishi, S., Fujii, N., Fusai, T., et al. (1999). Plasmodium falciparum domain mediating adhesion to chondroitin sulfate A: a receptor for human placental infection, Proc Natl Acad Sci U S A 96, 12743-8.

Bull, P. C., Lowe, B. S., Kortok, M., Molyneux, C. S., Newbold, C. I., and Marsh, K. (1998). Parasite antigens on the infected red cell surface are targets for naturally acquired immunity to malaria, Nat Med 4, 358-60.

Carcy, B., Bonnefoy, S., Guillotte, M., Le Scanf, C., Grellier, P., Schrevel, J., Fandeur, T., and Mercereau-Puijalon, O. (1994). A large multigene family expressed during the erythrocytic schizogony of Plasmodium falciparum, Mol Biochem Parasitol 68, 221-33.

Carlson, J., Helmby, H., Hill, A. V., Brewster, D., Greenwood, B. M., and Wahlgren, M. (1990). Human cerebral malaria: association with erythrocyte rosetting and lack of anti-rosetting antibodies, Lancet 336, 1457-60.

Carlson, J., and Wahlgren, M. (1992). Plasmodium falciparum erythrocyte rosetting is mediated by promiscuous lectin-like interactions, $J \operatorname{Exp} M e d$ 176, 1311-7. 
Chardin, P., and McCormick, F. (1999). Brefeldin A: the advantage of being uncompetitive, Cell 97, 153-5.

Chen, Q., Barragan, A., Fernandez, V., Sundstrom, A., Schlichtherle, M., Sahlen, A., Carlson, J., Datta, S., and Wahlgren, M. (1998a). Identification of Plasmodium falciparum erythrocyte membrane protein 1 (PfEMP1) as the rosetting ligand of the malaria parasite P. falciparum, $J$ Exp Med 187, 15-23.

Chen, Q., Fernandez, V., Sundstrom, A., Schlichtherle, M., Datta, S., Hagblom, P., and Wahlgren, M. (1998b). Developmental selection of var gene expression in Plasmodium falciparum, Nature 394, 392-5.

Chen, Q., Heddini, A., Barragan, A., Fernandez, V., Pearce, S. F., and Wahlgren, M. (2000). The semiconserved head structure of Plasmodium falciparum erythrocyte membrane protein 1 mediates binding to multiple independent host receptors, $J$ Exp Med 192, 1-10.

Cheng, Q., Cloonan, N., Fischer, K., Thompson, J., Waine, G., Lanzer, M., and Saul, A. (1998). stevor and rif are Plasmodium falciparum multicopy gene families which potentially encode variant antigens, Mol Biochem Parasitol 97, 161-76.

Clark, J. M. (1988). Novel non-templated nucleotide addition reactions catalyzed by procaryotic and eucaryotic DNA polymerases, Nucleic Acids Res 16, 9677-86.

Clarke, L., and Carbon, J. (1976). A colony bank containing synthetic Col El hybrid plasmids representative of the entire E. coli genome, Cell 9, 91-9.

Clough, B., Atilola, F. A., and Pasvoi, G. (1998). The role of rosetting in the multiplication of Plasmodium falciparum: rosette formation neither enhances nor targets parasite invasion into uninfected red cells, Br J Haematol 100, 99-104.

Clyde, D. F., Most, H., McCarthy, V. C., and Vanderberg, J. P. (1973). Immunization of man against sporozite-induced falciparum malaria, Am J Med Sci 266, 169-77. 
Cox, H. W. (1962). The behaviour of Plasmodium berghei strains isolated from relapsed infections of white mice., Journal of Protozoology 9, 114-118.

Crabb, B. S., Cooke, B. M., Reeder, J. C., Waller, R. F., Caruana, S. R., Davern, K. M., Wickham, M. E., Brown, G. V., Coppel, R. L., and Cowman, A. F. (1997). Targeted gene disruption shows that knobs enable malaria-infected red cells to cytoadhere under physiological shear stress, Cell 89, 287-96.

Craig, A. (2000). Malaria: a new gene family (clag) involved in adhesion, Parasitol Today 16, 366-7; discussion 405.

Craik, R. (1920). A note on the erythrocytes in malaria, The Lancet 1, 1110.

Crandall, I., and Sherman, I. W. (1991). Plasmodium falciparum (human malaria)induced modifications in human erythrocyte band 3 protein, Parasitology 102, 33540.

Crandall, I., and Sherman, I. W. (1996). The human anion transport protein, band 3, contains a CD36-like binding domain for Plasmodium falciparum-infected erythrocytes, Parasitology 112, 261-7.

D'Alessandro, U., Olaleye, B. O., McGuire, W., Langerock, P., Bennett, S., Aikins, M. K., Thomson, M. C., Cham, M. K., Cham, B. A., and Greenwood, B. M. (1995). Mortality and morbidity from malaria in Gambian children after introduction of an impregnated bednet programme, Lancet 345, 479-83.

Darkin-Rattray, S. J., Gurnett, A. M., Myers, R. W., Dulski, P. M., Crumley, T. M., Allocco, J. J., Cannova, C., Meinke, P. T., Colletti, S. L., Bednarek, M. A., et al. (1996). Apicidin: a novel antiprotozoal agent that inhibits parasite histone deacetylase, Proc Natl Acad Sci U S A 93, 13143-7.

Day, K. P., Hayward, R. E., and Dyer, M. (1998a). The biology of Plasmodium falciparum transmission stages, Parasitology 116, S95-109. 
Day, K. P., Hayward, R. E., Smith, D., and Culvenor, J. G. (1998b). CD36dependent adhesion and knob expression of the transmission stages of Plasmodium falciparum is stage specific, Mol Biochem Parasitol 93, 167-77.

Deitsch, K. W., Calderwood, M. S., and Wellems, T. E. (2001). MalariaCooperative silencing elements in var genes, Nature 412, 875-6.

Deitsch, K. W., Moxon, E. R., and Wellems, T. E. (1997). Shared themes of antigenic variation and virulence in bacterial, protozoal, and fungal infections, Microbiol Mol Biol Rev 61, 281-93.

del Portillo, H. A., Fernandez-Becerra, C., Bowman, S., Oliver, K., Preuss, M., Sanchez, C. P., Schneider, N. K., Villalobos, J. M., Rajandream, M. A., Harris, D., et al. (2001). A superfamily of variant genes encoded in the subtelomeric region of Plasmodium vivax, Nature 410, 839-42.

Delbac, F., Sanger, A., Neuhaus, E. M., Stratmann, R., Ajioka, J. W., Toursel, C., Herm-Gotz, A., Tomavo, S., Soldati, T., and Soldati, D. (2001). Toxoplasma gondii myosins B/C: one gene, two tails, two localizations, and a role in parasite division, $J$ Cell Biol 155, 613-24.

Dodge, J. T., Mitchell, C. and Hanahan, D.J. (1963). The preparation and chemical characterisation of haemoglobin-free ghosts of human erythrocytes., Archives of Biochemistry and Biophysics 100.

Doolan, D. L., and Hoffman, S. L. (2001). DNA-based vaccines against malaria: status and promise of the Multi-Stage Malaria DNA Vaccine Operation, Int $J$ Parasitol 31, 753-62.

Egan, A. F., Blackman, M. J., and Kaslow, D. C. (2000). Vaccine efficacy of recombinant Plasmodium falciparum merozoite surface protein 1 in malaria-naive, exposed, and/or -rechallenged Aotus vociferans monkeys, Infect Immun 68, 141827. 
Elford, B. C., Cowan, G. M., and Ferguson, D. J. (1995). Parasite-regulated membrane transport processes and metabolic control in malaria-infected erythrocytes, Biochem $J 308,361-74$.

Elmendorf, H. G., and Haldar, K. (1993). Identification and localization of ERD2 in the malaria parasite Plasmodium falciparum: separation from sites of sphingomyelin synthesis and implications for organization of the Golgi, Embo J 12, 4763-73.

Etzion, Z., and Perkins, M. E. (1989). Localization of a parasite encoded protein to erythrocyte cytoplasmic vesicles of Plasmodium falciparum-infected cells, Eur J Cell Biol 48, 174-9.

Farnert, A., Snounou, G., Rooth, I., and Bjorkman, A. (1997). Daily dynamics of Plasmodium falciparum subpopulations in asymptomatic children in a holoendemic area, Am J Trop Med Hyg 56, 538-47.

Fernandez, V., Hommel, M., Chen, Q., Hagblom, P., and Wahlgren, M. (1999). Small, clonally variant antigens expressed on the surface of the Plasmodium falciparum-infected erythrocyte are encoded by the rif gene family and are the target of human immune responses, J Exp Med 190, 1393-404.

Ferry, G. (2000). First DNA malaria vaccine on trial in Africa, Curr Biol 10, R8101.

Figueiredo, L. M., Pirritt, L. A., and Scherf, A. (2000). Genomic organisation and chromatin structure of Plasmodium falciparum chromosome ends, Mol Biochem Parasitol 106, 169-74.

Fischer, K., Horrocks, P., Preuss, M., Wiesner, J., Wunsch, S., Camargo, A. A., and Lanzer, M. (1997). Expression of var genes located within polymorphic subtelomeric domains of Plasmodium falciparum chromosomes, Molecular \& Cellular Biology 17, 3679-86. 
Foley, M., and Tilley, L. (1998). Protein trafficking in malaria-infected erythrocytes, Int J Parasitol 28, 1671-80.

Foo, A., Carter, R., Lambros, C., Graves, P., Quakyi, I., Targett, G. A., Ponnudurai, T., and Lewis, G. E., Jr. (1991). Conserved and variant epitopes of target antigens of transmission-blocking antibodies among isolates of Plasmodium falciparum from Malaysia, Am J Trop Med Hyg 44, 623-31.

Freeman, R. R., Trejdosiewicz, A. J., and Cross, G. A. (1980). Protective monoclonal antibodies recognising stage-specific merozoite antigens of a rodent malaria parasite, Nature 284, 366-8.

Freitas-Junior, L. H., Bottius, E., Pirrit, L. A., Deitsch, K. W., Scheidig, C., Guinet, F., Nehrbass, U., Wellems, T. E., and Scherf, A. (2000). Frequent ectopic recombination of virulence factor genes in telomeric chromosome clusters of $P$. falciparum, Nature 407, 1018-22.

Fried, M., and Duffy, P. E. (1996). Adherence of Plasmodium falciparum to chondroitin sulfate A in the human placenta, Science 272, 1502-4.

Gamain, B., Miller, L. H., and Baruch, D. I. (2001). The surface variant antigens of Plasmodium falciparum contain cross-reactive epitopes, Proc Natl Acad Sci US A 98, 2664-9.

Gardner, M. J., Tettelin, H., Carucci, D. J., Cummings, L. M., Aravind, L., Koonin, E. V., Shallom, S., Mason, T., Yu, K., Fujii, C., et al. (1998). Chromosome 2 sequence of the human malaria parasite Plasmodium falciparum [published erratum appears in Science 1998 Dec 4;282(5395):1827], Science 282, 1126-32.

Garnham, P. C. (1973). Recent research on malaria in mammals excluding man, $A d v$ Parasitol 11, 603-30.

Garrett, L. (1994). The Coming Plague (New York, Penguin Books). 
Gilles, H., and Warrel, D. (1993). Bruce-Chwatt's Essential Malariology, Third edn ( London, Edward Arnold).

Gommers-Ampt, J. H., Van Leeuwen, F., de Beer, A. L., Vliegenthart, J. F., Dizdaroglu, M., Kowalak, J. A., Crain, P. F., and Borst, P. (1993). beta-D-glucosylhydroxymethyluracil: a novel modified base present in the DNA of the parasitic protozoan T. brucei, Cell 75, 1129-36.

Greenwood, B. M., Bradley-Moore, A. M., Bryceson, A. D., and Palit, A. (1972). Immunosuppression in children with malaria, Lancet 1, 169-72.

Gruner, A. C., Brahimi, K., Eling, W., Konings, R., Meis, J., Aikawa, M., Daubersies, P., Guerin-Marchand, C., Mellouk, S., Snounou, G., and Druilhe, P. (2001). The Plasmodium falciparum knob-associated PfEMP3 antigen is also expressed at pre-erythrocytic stages and induces antibodies which inhibit sporozoite invasion, Mol Biochem Parasitol 112, 253-261.

Handunnetti, S. M., Mendis, K. N., and David, P. H. (1987). Antigenic variation of cloned Plasmodium fragile in its natural host Macaca sinica. Sequential appearance of successive variant antigenic types, $J \operatorname{Exp} \operatorname{Med} 165,1269-83$.

Handunnetti, S. M., van Schravendijk, M. R., Hasler, T., Barnwell, J. W., Greenwalt, D. E., and Howard, R. J. (1992). Involvement of CD36 on erythrocytes

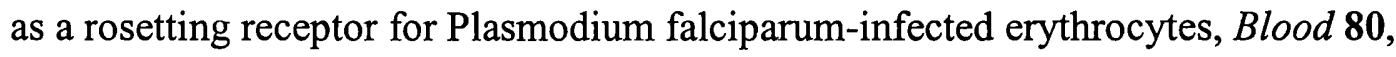
2097-104.

Harlow, E., and Lane, D. (1988). Antibodies: a laboratory manual, First edn (Cold Spring Harbour, Cold Spring Harbour Press).

Hayward, R. E., Tiwari, B., Piper, K. P., Baruch, D. I., and Day, K. P. (1999). Virulence and transmission success of the malarial parasite Plasmodium falciparum, Proceedings of the National Academy of Sciences of the United States of America 96, 4563-8. 
Heddini, A., Pettersson, F., Kai, O., Shafi, J., Obiero, J., Chen, Q., Barragan, A., Wahlgren, M., and Marsh, K. (2001). Fresh isolates from children with severe Plasmodium falciparum malaria bind to multiple receptors, Infect Immun 69, 584956.

Helmby, H., Cavelier, L., Pettersson, U., and Wahlgren, M. (1993). Rosetting Plasmodium falciparum-infected erythrocytes express unique strain-specific antigens on their surface, Infect Immun 61, 284-8.

Hernandez-Rivas, R., Mattei, D., Sterkers, Y., Peterson, D. S., Wellems, T. E., and Scherf, A. (1997). Expressed var genes are found in Plasmodium falciparum subtelomeric regions, Mol Cell Biol 17, 604-11.

Herrington, D. A., Clyde, D. F., Losonsky, G., Cortesia, M., Murphy, J. R., Davis, J., Baqar, S., Felix, A. M., Heimer, E. P., Gillessen, D., and et al. (1987). Safety and immunogenicity in man of a synthetic peptide malaria vaccine against Plasmodium falciparum sporozoites, Nature 328, 257-9.

Hill, A. V., Allsopp, C. E., Kwiatkowski, D., Anstey, N. M., Twumasi, P., Rowe, P. A., Bennett, S., Brewster, D., McMichael, A. J., and Greenwood, B. M. (1991). Common west African HLA antigens are associated with protection from severe malaria, Nature 352, 595-600.

Hill, A. V., Elvin, J., Willis, A. C., Aidoo, M., Allsopp, C. E., Gotch, F. M., Gao, X. M., Takiguchi, M., Greenwood, B. M., Townsend, A. R., and et al. (1992). Molecular analysis of the association of HLA-B53 and resistance to severe malaria, Nature 360, 434-9.

Hinterberg, K., Scherf, A., Gysin, J., Toyoshima, T., Aikawa, M., Mazie, J. C., da Silva, L. P., and Mattei, D. (1994). Plasmodium falciparum: the Pf332 antigen is secreted from the parasite by a brefeldin A-dependent pathway and is translocated to the erythrocyte membrane via the Maurer's clefts, Exp Parasitol 79, 279-91. 
Ho, M., Webster, H. K., Looareesuwan, S., Supanaranond, W., Phillips, R. E., Chanthavanich, P., and Warrell, D. A. (1986). Antigen-specific immunosuppression in human malaria due to Plasmodium falciparum, $J$ Infect Dis 153, 763-71.

Hoffman, S. L., Wistar, R., Jr., Ballou, W. R., Hollingdale, M. R., Wirtz, R. A., Schneider, I., Marwoto, H. A., and Hockmeyer, W. T. (1986). Immunity to malaria and naturally acquired antibodies to the circumsporozoite protein of Plasmodium falciparum, New England Journal of Medicine 315, 601-6.

Holder, A. A. (1999). Malaria vaccines, Proc Natl Acad Sci U S A 96, 1167-9. Holder, A. A., Blackman, M. J., Borre, M., Burghaus, P. A., Chappel, J. A., Keen, J. K., Ling, I. T., Ogun, S. A., Owen, C. A., and Sinha, K. A. (1994). Malaria parasites and erythrocyte invasion, Biochem Soc Trans 22, 291-5.

Holder, A. A., and Freeman, R. R. (1981). Immunization against blood-stage rodent malaria using purified parasite antigens, Nature 294, 361-4.

Hommel, M., David, P. H., and Oligino, L. D. (1983). Surface alterations of erythrocytes in Plasmodium falciparum malaria. Antigenic variation, antigenic diversity, and the role of the spleen, $J \operatorname{Exp}$ Med 157, 1137-48.

Howard, R. J., Barnwell, J. W., and Kao, V. (1983). Antigenic variation of Plasmodium knowlesi malaria: identification of the variant antigen on infected erythrocytes, Proc Natl Acad Sci U S A 80, 4129-33.

Howard, R. J., McBride, J. S., Aley, S. B., and Marsh, K. (1986). Antigenic diversity and size diversity of $\mathrm{P}$. falciparum antigens in isolates from Gambian patients. II. the schizont surface glycoprotein of molecular weight approximately 200 000, Parasite Immunol 8, 57-68.

Huestis, R., Cloonan, N., Tchavtchitch, M., and Saul, A. (2001). An algorithm to predict 3 ' intron splice sites in Plasmodium falciparum genomic sequences, $\mathrm{Mol}$ Biochem Parasitol 112, 71-77. 
Hui, G. S., and Siddiqui, W. A. (1988). Characterization of a Plasmodium falciparum polypeptide associated with membrane vesicles in the infected erythrocytes, Mol Biochem Parasitol 29, 283-93.

Janeway, C., Travers, P., Walport, M., and Capra, J. (1999). Immunobiology: The Immune System in Health and Disease, Fourth edn (New York, Garland Publishing, Inc.).

Jarra, W., and Brown, K. N. (1989). Invasion of mature and immature erythrocytes of CBA/Ca mice by a cloned line of Plasmodium chabaudi chabaudi, Parasitology 99, 157-63.

Jayaraman, K. S. (1997). India plans $\$ 200$ million attack on malaria, Nature 386, 536.

Jongwutiwes, S., Tanabe, K., Hughes, M. K., Kanbara, H., and Hughes, A. L. (1994). Allelic variation in the circumsporozoite protein of Plasmodium falciparum from Thai field isolates, Am J Trop Med Hyg 51, 659-68.

Kaul, D. K., Roth, E. F., Jr., Nagel, R. L., Howard, R. J., and Handunnetti, S. M. (1991). Rosetting of Plasmodium falciparum-infected red blood cells with uninfected red blood cells enhances microvascular obstruction under flow conditions, Blood 78, 812-9.

Keen, J., Holder, A., Playfair, J., Lockyer, M., and Lewis, A. (1990). Identification of the gene for a Plasmodium yoelii rhoptry protein. Multiple copies in the parasite genome, Mol Biochem Parasitol 42, 241-6.

Khan, S. M., Jarra, W., and Preiser, P. R. (2001). The $235 \mathrm{kDa}$ rhoptry protein of Plasmodium (yoelii) yoelii: function at the junction, Mol Biochem Parasitol 117, 110. 
Kilejian, A. (1980). Homology between a histidine-rich protein from Plasmodium lophurae and a protein associated with the knob-like protrusions on membranes of erythrocytes infected with Plasmodium falciparum, $J$ Exp Med 151, 1534-8.

Kirk, K., Tilley, L., and Ginsburg, H. (1999). Transport and trafficking in the malaria-infected erythrocyte, Parasitol Today 15, 355-7.

Kitchen, S. (1938). The infection of reticulocytes by Plasmodium vivax, American Journal of Tropical Medicine \& Hygiene 18, 347-359.

Kumar, N., Koski, G., Harada, M., Aikawa, M., and Zheng, H. (1991). Induction and localization of Plasmodium falciparum stress proteins related to the heat shock protein 70 family, Mol Biochem Parasitol 48, 47-58.

Kumaratilake, L. M., Ferrante, A., Jaeger, T., and Rzepczyk, C. M. (1992). Effects of cytokines, complement, and antibody on the neutrophil respiratory burst and phagocytic response to Plasmodium falciparum merozoites, Infect Immun 60, 37318.

Kutner, S., Breuer, W. V., Ginsburg, H., Aley, S. B., and Cabantchik, Z. I. (1985). Characterization of permeation pathways in the plasma membrane of human erythrocytes infected with early stages of Plasmodium falciparum: association with parasite development, J Cell Physiol 125, 521-7.

Kwiatkowski, D. (1989). Febrile temperatures can synchronize the growth of Plasmodium falciparum in vitro, $J \operatorname{Exp} M e d$ 169, 357-61.

Kwiatkowski, D., Cannon, J. G., Manogue, K. R., Cerami, A., Dinarello, C. A., and Greenwood, B. M. (1989). Tumour necrosis factor production in Falciparum malaria and its association with schizont rupture, Clin Exp Immunol 77, 361-6.

Kwiatkowski, D., Hill, A. V., Sambou, I., Twumasi, P., Castracane, J., Manogue, K. R., Cerami, A., Brewster, D. R., and Greenwood, B. M. (1990). TNF concentration 
in fatal cerebral, non-fatal cerebral, and uncomplicated Plasmodium falciparum malaria, Lancet 336, 1201-4.

Kwiatkowski, D., Molyneux, M. E., Stephens, S., Curtis, N., Klein, N., Pointaire, P., Smit, M., Allan, R., Brewster, D. R., Grau, G. E., and et al. (1993). Anti-TNF therapy inhibits fever in cerebral malaria, $Q J$ Med 86, 91-8.

Kyes, S., Horrocks, P., and Newbold, C. (2001). Antigenic variation at the infected red cell surface in malaria, Annu Rev Microbiol 55, 673-707.

Kyes, S., Pinches, R., and Newbold, C. (2000). A simple RNA analysis method shows var and rif multigene family expression patterns in Plasmodium falciparum, Molecular \& Biochemical Parasitology 105, 311-5.

Kyes, S. A., Rowe, J. A., Kriek, N., and Newbold, C. I. (1999). Rifins: a second family of clonally variant proteins expressed on the surface of red cells infected with Plasmodium falciparum, Proceedings of the National Academy of Sciences of the United States of America 96, 9333-8.

Lalvani, A., and Hill, A. V. (1998). Cytotoxic T-lymphocytes against malaria and tuberculosis: from natural immunity to vaccine design, Clinical Science 95, 531-8. Lambros, C., and Vanderberg, J. P. (1979). Synchronization of Plasmodium falciparum erythrocytic stages in culture, $J$ Parasitol 65, 418-20.

Langhorne, J., Cross, C., Seixas, E., Li, C., and von der Weid, T. (1998). A role for $\mathrm{B}$ cells in the development of $\mathrm{T}$ cell helper function in a malaria infection in mice, Proc Natl Acad Sci U S A 95, 1730-4.

Langhorne, J., Gillard, S., Simon, B., Slade, S., and Eichmann, K. (1989).

Frequencies of CD4+ T cells reactive with Plasmodium chabaudi chabaudi: distinct response kinetics for cells with Th1 and Th2 characteristics during infection, Int Immunol 1, 416-24. 
Langhorne, J., Simon-Haarhaus, B., and Meding, S. J. (1990). The role of CD4+ T cells in the protective immune response to Plasmodium chabaudi in vivo, Immunol Lett 25, 101-7.

Leech, J. H., Barnwell, J. W., Miller, L. H., and Howard, R. J. (1984). Identification of a strain-specific malarial antigen exposed on the surface of Plasmodium falciparum-infected erythrocytes, $J$ Exp Med 159, 1567-75.

Lell, B., May, J., Schmidt-Ott, R. J., Lehman, L. G., Luckner, D., Greve, B., Matousek, P., Schmid, D., Herbich, K., Mockenhaupt, F. P., et al. (1999). The role of red blood cell polymorphisms in resistance and susceptibility to malaria, Clin Infect Dis 28, 794-9.

Lew, V. L. (2001). Packaged merozoite release without immediate host cell lysis, Trends Parasitol 17, 401-3.

Lewin, B. (1997). Genes VI, 6th edn (Oxford, Oxford University Press).

Limpaiboon, T., Shirley, M. W., Kemp, D. J., and Saul, A. (1991). 7H8/6, a multicopy DNA probe for distinguishing isolates of Plasmodium falciparum, $\mathrm{Mol}$ Biochem Parasitol 47, 197-206.

Lucas, J. Z., and Sherman, I. W. (1998). Plasmodium falciparum: thrombospondin mediates parasitized erythrocyte band 3-related adhesin binding, Exp Parasitol 89, 78-85.

MacPherson, G. G., Warrell, M. J., White, N. J., Looareesuwan, S., and Warrell, D. A. (1985). Human cerebral malaria. A quantitative ultrastructural analysis of parasitized erythrocyte sequestration, Am J Pathol 119, 385-401.

Marsh, K., Otoo, L., Hayes, R. J., Carson, D. C., and Greenwood, B. M. (1989). Antibodies to blood stage antigens of Plasmodium falciparum in rural Gambians and their relation to protection against infection, Trans $R$ Soc Trop Med Hyg 83, 293303. 
Martinez, S. L., Clavijo, C. A., and Winograd, E. (1998). Identification of peripheral membrane proteins associated with the tubo-vesicular network of Plasmodium falciparum infected erythrocytes, Mol Biochem Parasitol 91, 273-80.

Mattei, D., Berry, L., Couffin, S., and Richard, O. (1999). The transport of the histidine-rich protein I from Plasmodium falciparum is insensitive to brefeldin A, Novartis Found Symp 226, 215-26; discussion 227-30.

Maubert, B., Fievet, N., Tami, G., Cot, M., Boudin, C., and Deloron, P. (1999). Development of antibodies against chondroitin sulfate A-adherent Plasmodium falciparum in pregnant women, Infect Immun 67, 5367-71.

McColl, D. J., and Anders, R. F. (1997). Conservation of structural motifs and antigenic diversity in the Plasmodium falciparum merozoite surface protein-3 (MSP3), Mol Biochem Parasitol 90, 21-31.

McGuire, W., Hill, A. V., Allsopp, C. E., Greenwood, B. M., and Kwiatkowski, D. (1994). Variation in the TNF-alpha promoter region associated with susceptibility to cerebral malaria, Nature 371, 508-10.

McLean, S. A., Pearson, C. D., and Phillips, R. S. (1982). Plasmodium chabaudi: antigenic variation during recrudescent parasitaemias in mice, Exp Parasitol 54, 296-302.

Mendis, K. N., Ihalamulla, R. I., and David, P. H. (1988). Diversity of Plasmodium vivax-induced antigens on the surface of infected human erythrocytes, Am J Trop Med Hyg 38, 42-6.

Menendez, C. (1995). Malaria during pregnancy: A priority area of malaria research and control, Parasitology Today 11, 178-183.

Menendez, C., Fleming, A. F., and Alonso, P. L. (2000). Malaria-related anaemia, Parasitol Today 16, 469-76. 
Miller, L. H., Mason, S. J., Clyde, D. F., and McGinniss, M. H. (1976). The resistance factor to Plasmodium vivax in blacks. The Duffy-blood-group genotype, FyFy, $N$ Engl J Med 295, 302-4.

Miller, L. H., Mason, S. J., Dvorak, J. A., McGinniss, M. H., and Rothman, I. K. (1975). Erythrocyte receptors for (Plasmodium knowlesi) malaria: Duffy blood group determinants, Science 189, 561-3.

Miller, L. H., Roberts, T., Shahabuddin, M., and McCutchan, T. F. (1993). Analysis of sequence diversity in the Plasmodium falciparum merozoite surface protein-1 (MSP-1), Mol Biochem Parasitol 59, 1-14.

Nacer, A., Berry, L., Slomianny, C., and Mattei, D. (2001). Plasmodium falciparum signal sequences: simply sequences or special signals?, Int J Parasitol 31, 1371-9.

Nagel, R. L. (1990). Innate resistance to malaria: the intraerythrocytic cycle, Blood Cells 16, 321-39; discussion 340-9.

Newbold, C., Warn, P., Black, G., Berendt, A., Craig, A., Snow, B., Msobo, M., Peshu, N., and Marsh, K. (1997a). Receptor-specific adhesion and clinical disease in Plasmodium falciparum, Am J Trop Med Hyg 57, 389-98.

Newbold, C. I., Craig, A. G., Kyes, S., Berendt, A. R., Snow, R. W., Peshu, N., and Marsh, K. (1997b). PfEMP1, polymorphism and pathogenesis, Ann Trop Med Parasitol 91, 551-7.

Noviyanti, R., Brown, G. V., Wickham, M. E., Duffy, M. F., Cowman, A. F., and Reeder, J. C. (2001). Multiple var gene transcripts are expressed in Plasmodium falciparum infected erythrocytes selected for adhesion, Molecular \& Biochemical Parasitology 114, 227-37. 
Nussenzweig, R. S., Vanderberg, J., Most, H., and Orton, C. (1967). Protective immunity produced by the injection of $\mathrm{x}$-irradiated sporozoites of plasmodium berghei, Nature 216, 160-2.

Nussler, A., Pied, S., Goma, J., Renia, L., Miltgen, F., Grau, G. E., and Mazier, D. (1991). TNF inhibits malaria hepatic stages in vitro via synthesis of IL-6, Int Immunol 3, 317-21.

Ockenhouse, C. F., Klotz, F. W., Tandon, N. N., and Jamieson, G. A. (1991).

Sequestrin, a CD36 recognition protein on Plasmodium falciparum malaria-infected erythrocytes identified by anti-idiotype antibodies, Proc Natl Acad Sci U S A 88, 3175-9.

Ogun, S. A., and Holder, A. A. (1996). A high molecular mass Plasmodium yoelii rhoptry protein binds to erythrocytes, Mol Biochem Parasitol 76, 321-4.

Oka, M., Aikawa,, M., Freeman, R.R., Holder, A.A., and Fine, E. (1984).

Ultrastructural localization of protective antigens of Plasmodium yoelii merozoites by the use monoclonal antibodies and ultrathin cryomicrotomy, Am J Trop Med Hyg $33,342-6$.

Owen, C. A., Sinha, K. A., Keen, J. K., Ogun, S. A., and Holder, A. A. (1999).

Chromosomal organisation of a gene family encoding rhoptry proteins in Plasmodium yoelii, Mol Biochem Parasitol 99, 183-92.

Pachebat, J. A., Ling, I. T., Grainger, M., Trucco, C., Howell, S., Fernandez-Reyes, D., Gunaratne, R., and Holder, A. A. (2001). The $22 \mathrm{kDa}$ component of the protein complex on the surface of Plasmodium falciparum merozoites is derived from a larger precursor, merozoite surface protein 7, Mol Biochem Parasitol 117, 83-9.

Pasvol, G., Wilson, R. J., Smalley, M. E., and Brown, J. (1978). Separation of viable schizont-infected red cells of Plasmodium falciparum from human blood, Ann Trop Med Parasitol 72, 87-8. 
Perry, A. C., Hart, C. A., Nicolson, I. J., Heckels, J. E., and Saunders, J. R. (1987). Inter-strain homology of pilin gene sequences in Neisseria meningitidis isolates that express markedly different antigenic pilus types, J Gen Microbiol 133, 1409-18.

Peterson, D. S., Miller, L. H., and Wellems, T. E. (1995). Isolation of multiple sequences from the Plasmodium falciparum genome that encode conserved domains homologous to those in erythrocyte-binding proteins, Proc Natl Acad Sci US A 92, $7100-4$

Phillips, R. S., Brannan, L. R., Balmer, P., and Neuville, P. (1997). Antigenic variation during malaria infection--the contribution from the murine parasite Plasmodium chabaudi, Parasite Immunol 19, 427-34.

Pinder, J. C., Pekrun, A., Maggs, A. M., Brain, A. P., and Gratzer, W. B. (1995). Association state of human red blood cell band 3 and its interaction with ankyrin, Blood 85, 2951-61.

Piper, K. P., Hayward, R. E., Cox, M. J., and Day, K. P. (1999). Malaria transmission and naturally acquired immunity to PfEMP-1, Infection \& Immunity 67, 6369-74.

Pollack, Y., Kogan, N., and Golenser, J. (1991). Plasmodium falciparum: evidence for a DNA methylation pattern, Exp Parasitol 72, 339-44.

Pongponratn, E., Riganti, M., Punpoowong, B., and Aikawa, M. (1991). Microvascular sequestration of parasitized erythrocytes in human falciparum malaria: a pathological study, Am J Trop Med Hyg 44, 168-75.

Pouvelle, B., Buffet, P. A., Lepolard, C., Scherf, A., and Gysin, J. (2000). Cytoadhesion of Plasmodium falciparum ring-stage-infected erythrocytes, Nat Med 6, 1264-8.

Preiser, P. R., and Jarra, W. (1998). Plasmodium yoelii: differences in the transcription of the $235-\mathrm{kDa}$ rhoptry protein multigene family in lethal and nonlethal lines, Exp Parasitol 89, 50-7. 
Preiser, P. R., Jarra, W., Capiod, T., and Snounou, G. (1999). A rhoptry-proteinassociated mechanism of clonal phenotypic variation in rodent malaria [see comments], Nature 398, 618-22.

Reeder, J. C., Cowman, A. F., Davern, K. M., Beeson, J. G., Thompson, J. K., Rogerson, S. J., and Brown, G. V. (1999). The adhesion of Plasmodium falciparuminfected erythrocytes to chondroitin sulfate $\mathrm{A}$ is mediated by $\mathrm{P}$. falciparum erythrocyte membrane protein 1, Proc Natl Acad Sci U S A 96, 5198-202.

Riley, E. M., Andersson, G., Otoo, L. N., Jepsen, S., and Greenwood, B. M. (1988). Cellular immune responses to Plasmodium falciparum antigens in Gambian children during and after an acute attack of falciparum malaria, Clin Exp Immunol 73, 17-22.

Roberts, D. J., Craig, A. G., Berendt, A. R., Pinches, R., Nash, G., Marsh, K., and Newbold, C. I. (1992). Rapid switching to multiple antigenic and adhesive phenotypes in malaria [see comments], Nature 357, 689-92.

Rogers, N. J., Hall, B. S., Obiero, J., Targett, G. A., and Sutherland, C. J. (2000). A model for sequestration of the transmission stages of Plasmodium falciparum: adhesion of gametocyte-infected erythrocytes to human bone marrow cells, Infect Immun 68, 3455-62.

Rogerson, S. J., Tembenu, R., Dobano, C., Plitt, S., Taylor, T. E., and Molyneux, M. E. (1999). Cytoadherence characteristics of Plasmodium falciparum-infected erythrocytes from Malawian children with severe and uncomplicated malaria, $A m J$ Trop Med Hyg 61, 467-72.

Rowe, A., Berendt, A. R., Marsh, K., and Newbold, C. I. (1994). Plasmodium falciparum: a family of sulphated glycoconjugates disrupts erythrocyte rosettes, Exp Parasitol 79, 506-16.

Rowe, A., Obeiro, J., Newbold, C. I., and Marsh, K. (1995). Plasmodium falciparum rosetting is associated with malaria severity in Kenya, Infect Immun 63, 2323-6. 
Rowe, J. A., Moulds, J. M., Newbold, C. I., and Miller, L. H. (1997). P. falciparum rosetting mediated by a parasite-variant erythrocyte membrane protein and complement-receptor 1, Nature 388, 292-5.

Rowe, J. A., Rogerson, S. J., Raza, A., Moulds, J. M., Kazatchkine, M. D., Marsh, K., Newbold, C. I., Atkinson, J. P., and Miller, L. H. (2000). Mapping of the region of complement receptor (CR) 1 required for Plasmodium falciparum rosetting and demonstration of the importance of CR1 in rosetting in field isolates, $J$ Immunol $165,6341-6$.

Rubio, J. P., Thompson, J. K., and Cowman, A. F. (1996). The var genes of Plasmodium falciparum are located in the subtelomeric region of most chromosomes, Embo J 15, 4069-77.

Rudenko, G. (1999). Genes involved in phenotypic and antigenic variation in African trypanosomes and malaria, Curr Opin Microbiol 2, 651-6.

Saitou, N., and Nei, M. (1987). The neighbor-joining method: a new method for reconstructing phylogenetic trees, Mol Biol Evol 4, 406-25.

Sanger, F., Nicklen, S., and Coulson, A. R. (1977). DNA sequencing with chainterminating inhibitors, Proc Natl Acad Sci US A 74, 5463-7.

Saul, A. (1999). The role of variant surface antigens on malaria-infected red blood cells, Parasitol Today 15, 455-7.

Scherf, A., Hernandez-Rivas, R., Buffet, P., Bottius, E., Benatar, C., Pouvelle, B., Gysin, J., and Lanzer, M. (1998). Antigenic variation in malaria: in situ switching, relaxed and mutually exclusive transcription of var genes during intra-erythrocytic development in Plasmodium falciparum, EMBO Journal 17, 5418-26. 
Schofield, L., Villaquiran, J., Ferreira, A., Schellekens, H., Nussenzweig, R., and Nussenzweig, V. (1987). Gamma interferon, CD8+ T cells and antibodies required for immunity to malaria sporozoites, Nature 330, 664-6.

Shi, Y. P., Hasnain, S. E., Sacci, J. B., Holloway, B. P., Fujioka, H., Kumar, N., Wohlhueter, R., Hoffman, S. L., Collins, W. E., and Lal, A. A. (1999).

Immunogenicity and in vitro protective efficacy of a recombinant multistage Plasmodium falciparum candidate vaccine, Proc Natl Acad Sci U S A 96, 1615-20.

Shirley, M. W., Biggs, B. A., Forsyth, K. P., Brown, H. J., Thompson, J. K., Brown, G. V., and Kemp, D. J. (1990). Chromosome 9 from independent clones and isolates of Plasmodium falciparum undergoes subtelomeric deletions with similar breakpoints in vitro, Mol Biochem Parasitol 40, 137-45.

Smith, J. D., Chitnis, C. E., Craig, A. G., Roberts, D. J., Hudson-Taylor, D. E., Peterson, D. S., Pinches, R., Newbold, C. I., and Miller, L. H. (1995). Switches in expression of Plasmodium falciparum var genes correlate with changes in antigenic and cytoadherent phenotypes of infected erythrocytes [see comments], Cell 82, 10110.

Smith, J. D., Craig, A. G., Kriek, N., Hudson-Taylor, D., Kyes, S., Fagen, T., Pinches, R., Baruch, D. I., Newbold, C. I., and Miller, L. H. (2000). Identification of a Plasmodium falciparum intercellular adhesion molecule-1 binding domain: a parasite adhesion trait implicated in cerebral malaria, Proc Natl Acad Sci US A 97, 1766-71.

Smith, J. D., Kyes, S., Craig, A. G., Fagan, T., Hudson-Taylor, D., Miller, L. H., Baruch, D. I., and Newbold, C. I. (1998). Analysis of adhesive domains from the A4VAR Plasmodium falciparum erythrocyte membrane protein-1 identifies a CD36 binding domain, Mol Biochem Parasitol 97, 133-48.

Spencer, H. C., Miller, L. H., Collins, W. E., Knud-Hansen, C., McGinnis, M. H., Shiroishi, T., Lobos, R. A., and Feldman, R. A. (1978). The Duffy blood group and resistance to Plasmodium vivax in Honduras, Am J Trop Med Hyg 27, 664-70. 
Stevenson, M. M., and Ghadirian, E. (1989). Human recombinant tumor necrosis factor alpha protects susceptible $\mathrm{A} / \mathrm{J}$ mice against lethal Plasmodium chabaudi AS infection, Infect Immun 57, 3936-9.

Stoute, J. A., Kester, K. E., Krzych, U., Wellde, B. T., Hall, T., White, K., Glenn, G., Ockenhouse, C. F., Garcon, N., Schwenk, R., et al. (1998). Long-term efficacy and immune responses following immunization with the RTS,S malaria vaccine, $J$ Infect Dis 178, 1139-44.

Su, X. Z., Heatwole, V. M., Wertheimer, S. P., Guinet, F., Herrfeldt, J. A., Peterson, D. S., Ravetch, J. A., and Wellems, T. E. (1995). The large diverse gene family var encodes proteins involved in cytoadherence and antigenic variation of Plasmodium falciparum-infected erythrocytes [see comments], Cell 82, 89-100.

Sutherland, C. J. (2001). Stevor transcripts from Plasmodium falciparum gametocytes encode truncated polypeptides, Mol Biochem Parasitol 113, 331-5.

Taubes, G. (2000). Vaccines. Searching for a parasite's weak spot, Science 290, 4347.

Taylor, H. M., Kyes, S. A., Harris, D., Kriek, N., and Newbold, C. I. (2000a). A study of var gene transcription in vitro using universal var gene primers, Molecular \& Biochemical Parasitology 105, 13-23.

Taylor, H. M., Kyes, S. A., and Newbold, C. I. (2000b). Var gene diversity in Plasmodium falciparum is generated by frequent recombination events, $\mathrm{Mol}$ Biochem Parasitol 110, 391-7.

Thaithong, S., Beale, G. H., Fenton, B., McBride, J., Rosario, V., Walker, A., and Walliker, D. (1984). Clonal diversity in a single isolate of the malaria parasite Plasmodium falciparum, Trans $R$ Soc Trop Med Hyg 78, 242-5. 
Thompson, J. K., Rubio, J. P., Caruana, S., Brockman, A., Wickham, M. E., and Cowman, A. F. (1997). The chromosomal organization of the Plasmodium falciparum var gene family is conserved, Mol Biochem Parasitol 87, 49-60.

Trager, W., and Jensen, J. B. (1977). Cultivation of erythrocytic stages, Bull World Health Organ 55, 363-5.

Trenholme, K. R., Gardiner, D. L., Holt, D. C., Thomas, E. A., Cowman, A. F., and Kemp, D. J. (2000). clag9: A cytoadherence gene in Plasmodium falciparum essential for binding of parasitized erythrocytes to CD36, Proc Natl Acad Sci US A 97, 4029-33.

Trigg, P. I., and Kondrachine, A. V. (1998). The Current Global Malaria Situation. In Malaria: Parasite Biology, Pathogenesis and Protection, I. W. Sherman, ed. (ASM Press), pp. 11-22.

Urban, B. C., Ferguson, D. J., Pain, A., Willcox, N., Plebanski, M., Austyn, J. M., and Roberts, D. J. (1999). Plasmodium falciparum-infected erythrocytes modulate the maturation of dendritic cells [see comments], Nature 400, 73-7.

Urban, B. C., Willcox, N., and Roberts, D. J. (2001). A role for CD36 in the regulation of dendritic cell function, Proc Natl Acad Sci US A 98, 8750-5.

Vanhamme, L., Pays, E., McCulloch, R., and Barry, J. D. (2001). An update on antigenic variation in African trypanosomes, Trends Parasitol 17, 338-43.

Wahlgren, M., Carlson, J., Udomsangpetch, R., and Perlmann, P. (1989). Why do Plasmodium falciparum-infected erythrocytes form spontaneous erythrocyte rosettes?, Parasitology Today 5, 183-185.

Walliker, D., Carter, R., and Sanderson, A. (1975). Genetic studies on Plasmodium chabaudi: recombination between enzyme markers, Parasitology 70, 19-24. 
Walliker, D., Quakyi, I. A., Wellems, T. E., McCutchan, T. F., Szarfman, A., London, W. T., Corcoran, L. M., Burkot, T. R., and Carter, R. (1987). Genetic analysis of the human malaria parasite Plasmodium falciparum, Science 236, 1661 6.

Waterkeyn, J. G., Wickham, M. E., Davern, K. M., Cooke, B. M., Coppel, R. L., Reeder, J. C., Culvenor, J. G., Waller, R. F., and Cowman, A. F. (2000). Targeted mutagenesis of Plasmodium falciparum erythrocyte membrane protein 3 (PfEMP3) disrupts cytoadherence of malaria-infected red blood cells, Embo J 19, 2813-23.

Weber, J. L. (1988). Interspersed repetitive DNA from Plasmodium falciparum, $\mathrm{Mol}$ Biochem Parasitol 29, 117-24.

Webster, H. K., Ho, M., Looareesuwan, S., Pavanand, K., Wattanagoon, Y., Warrell, D. A., and Hockmeyer, W. T. (1988). Lymphocyte responsiveness to a candidate malaria sporozoite vaccine (R32tet32) of individuals with naturally acquired Plasmodium falciparum malaria, Am J Trop Med Hyg 38, 37-41.

Weiss, W. R., Mellouk, S., Houghten, R. A., Sedegah, M., Kumar, S., Good, M. F., Berzofsky, J. A., Miller, L. H., and Hoffman, S. L. (1990). Cytotoxic T cells recognize a peptide from the circumsporozoite protein on malaria-infected hepatocytes, $J$ Exp Med 171, 763-73.

White, K. L., Snyder, H. L., and Krzych, U. (1996). MHC class I-dependent presentation of exoerythrocytic antigens to $\mathrm{CD} 8+\mathrm{T}$ lymphocytes is required for protective immunity against Plasmodium berghei, J Immunol 156, 3374-81.

WHO (1999). Report on infectious diseases: Removing obstacles to healthy development, Published by WHO, Geneva).

Wickham, M. E., Rug, M., Ralph, S. A., Klonis, N., McFadden, G. I., Tilley, L., and Cowman, A. F. (2001). Trafficking and assembly of the cytoadherence complex in Plasmodium falciparum-infected human erythrocytes, Embo $J$ 20, 5636-5649. 
Winograd, E., Robles, W. M., Caldas, M. L., and Cortes, G. T. (2001).

Cytoadherence of the malaria-infected erythrocyte membrane to C32 melanoma cells after merozoites are released from parasitized infected cells, Parasitol Res 87, 264-8.

Winograd, E., and Sherman, I. W. (1989). Characterization of a modified red cell membrane protein expressed on erythrocytes infected with the human malaria parasite Plasmodium falciparum: possible role as a cytoadherent mediating protein, J Cell Biol 108, 23-30.

Wolffe, A. P. (1996). Histone deacetylase: a regulator of transcription [comment], Science 272, 371-2. 